\title{
Measurement of the Branching Fraction of the Semileptonic Decay Anti-B0 ---> D*+ Mu- Anti-Muon-Neutrino in the BaBar Experiment
}

\author{
by Douglas Michael Thiessen
}

Ph.D. Thesis 



\section{Measurement of the Branching Fraction of the Semileptonic Decay $\bar{B}^{0} \rightarrow D^{*+} \mu^{-} \bar{\nu}_{\mu}$ in the BABAR Experiment by}

Douglas Michael Thiessen

B.Sc., The University of British Columbia, 1993 M.Sc., Simon Fraser University, 1996

\section{A THESIS SUBMITTED IN PARTIAL FULFILMENT OF} THE REQUIREMENTS FOR THE DEGREE OF

\section{DOCTOR OF PHILOSOPHY}

in

The Faculty of Graduate Studies

(Department of Physics and Astronomy)

We accept this thesis as conforming to the required standard

\section{THE UNIVERSITY OF BRITISH COLUMBIA}

February 2004

(c) Douglas Michael Thiessen, 2004 


\section{Abstract}

This thesis presents a measurement of the branching fraction for the exclusive semileptonic decay $\bar{B}^{0} \rightarrow D^{*+} \mu^{-} \bar{\nu}_{\mu}$. This decay is essentially the decay of a $b$ quark to a $c$ quark inside the meson with the release of a muon and neutrino, and it provides a very clean signal for measuring various $B$ meson decay properties. The data for this measurement consist of 22.3 million $B \bar{B}$ pairs collected by the BABAR detector at the Stanford Linear Accelerator Center in 1999-2000. The branching fraction is measured to be $\mathcal{B}\left(\bar{B}^{0} \rightarrow\right.$ $\left.D^{*+} \mu^{-} \bar{\nu}_{\mu}\right)=[4.72 \pm 0.10$ (data stat.) \pm 0.06 (MC stat.) \pm 0.20 (syst.) \pm 0.17 (other $\mathcal{B}$ ) $] \%$. 


\section{Contents}

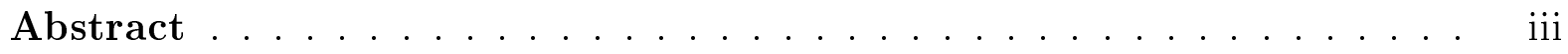

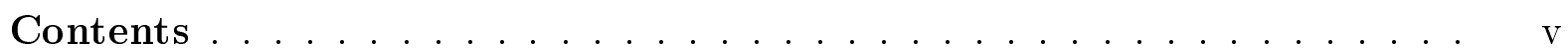

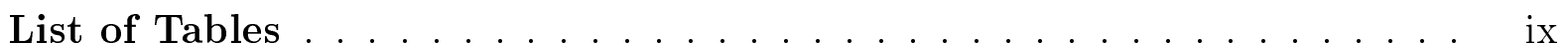

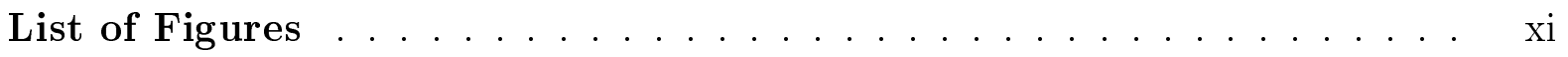

Dedication . . . . . . . . . . . . . . . xiii

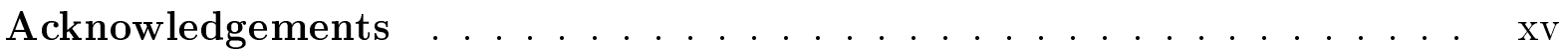

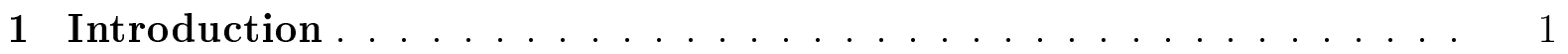

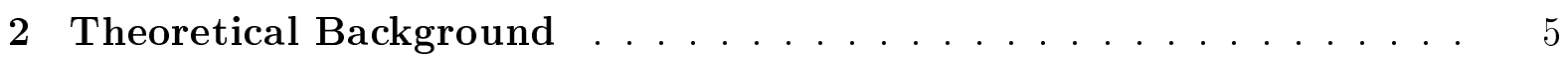

2.1 The Standard Model . . . . . . . . . . . . . . . . . . . . 5

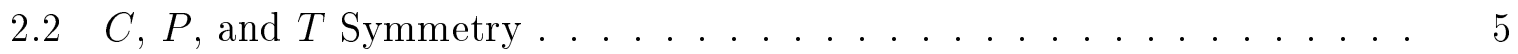

2.3 The CKM Matrix . . . . . . . . . . . . . 6

2.4 Heavy Quark Effective Theory . . . . . . . . . . . . . . . . . 8

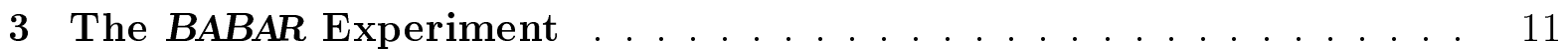

3.1 Overview . . . . . . . . . . . . . . . . . . . . . . 12

3.2 PEP-II . . . . . . . . . . . . . . . . . . . . . . . . . . . . . 13

3.3 Silicon Vertex Tracker . . . . . . . . . . . . . . . . . . . . . . 17

3.4 Drift Chamber . . . . . . . . . . . . . . . . . . . . . 19

3.5 Cherenkov Detector . . . . . . . . . . . . . . . . . . . . . 23

3.6 Electromagnetic Calorimeter . . . . . . . . . . . . . . . . . . 25

3.7 Main Solenoid . . . . . . . . . . . . . . . . . . . . . . . 28

3.8 Instrumented Flux Return . . . . . . . . . . . . . . . . . . . 30

3.8.1 IFR Efficiency Degradation . . . . . . . . . . . . 32

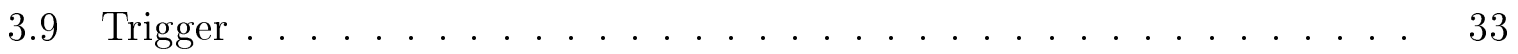

4 Charged Particle Tracking and Identification . . . . . . . . . . 35

4.1 Charged Particle Tracking . . . . . . . . . . . . . . . . . . . 35

4.1 .1 Reconstruction . . . . . . . . . . . . . 35

4.1 .2 Efficiency . . . . . . . . . . . . . 37

4.1.3 Track Parameter Resolution . . . . . . . . . . . . . . 37 
4.1.4 Tracking Lists . . . . . . . . . . . . . . . . 38

4.2 Particle Identification . . . . . . . . . . . . . . . . . . . . . . . . . . . . . . . . . . . . . . . .

4.2.1 Muon Identification . . . . . . . . . . . . . . . 40

4.2 .2 Charged Kaon Identification . . . . . . . . . . . . . . 42

4.2 .3 Electron Identification . . . . . . . . . . . . . . . . . 43

4.2.4 PID Control Samples . . . . . . . . . . . . . 43

5 Data and Monte Carlo Selection . . . . . . . . . . . . . . . . 45

5.1 BABAR Data . . . . . . . . . . . . . . . . . . . . . . . 45

5.2 Monte Carlo Simulated Data . . . . . . . . . . . . . . . . 46

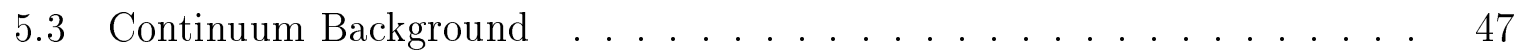

5.4 Event Reconstruction and Selection . . . . . . . . . . . . . . 48

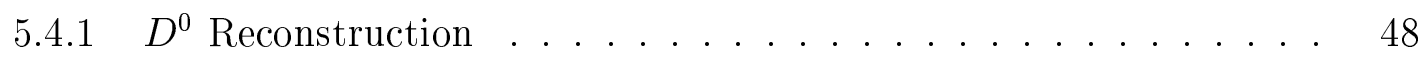

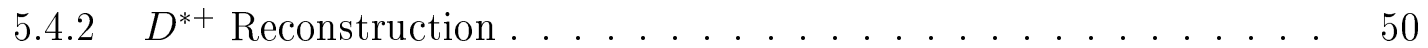

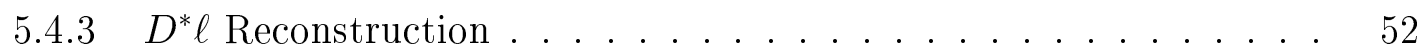

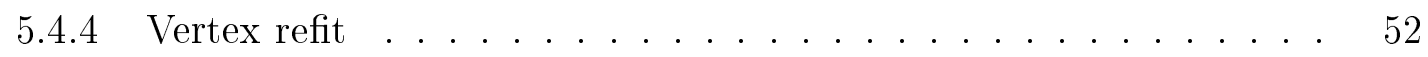

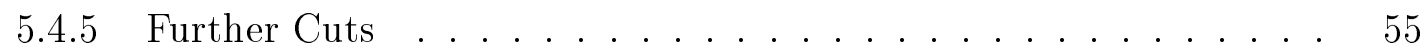

5.5 Monte Carlo Corrections . . . . . . . . . . . . . . . . 56

5.5.1 Tracking Efficiency Correction . . . . . . . . . . . . 56

5.5 .2 Slow $\pi$ Efficiency Correction . . . . . . . . . . . 56

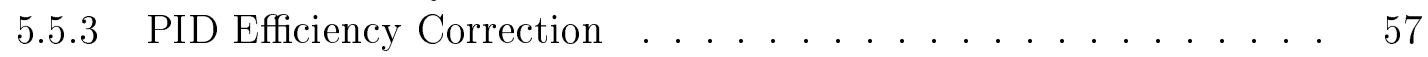

5.5.4 Beam Energy Correction .................. 58

6 Background Characterization . . . . . . . . . . . . . . 59

6.1 Monte Carlo Truth . . . . . . . . . . . . . . . . 60

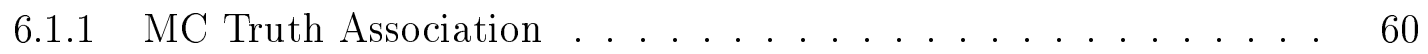

6.1 .2 MC Truth Variables .................. 60

$6.2 \cos \theta_{B, D^{*} \ell}$ Angular Variable . . . . . . . . . . . . . . . . . . . . . . . . . . . . . . 61

$6.3 \bar{B}^{0} \rightarrow D^{*+} \mu^{-} \bar{\nu}_{\mu}$ Signal . . . . . . . . . . . . . . . . . . . . 62

$6.4 B \rightarrow D^{*+} \mu^{-} \bar{\nu}_{\mu} \pi$ Background . . . . . . . . . . . . . 63

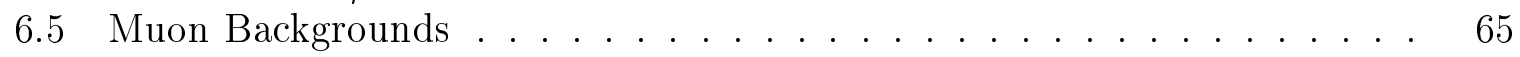

6.5.1 Correlated muons . . . . . . . . . . . . . . . . 66

6.5.2 Uncorrelated muons .................... 68

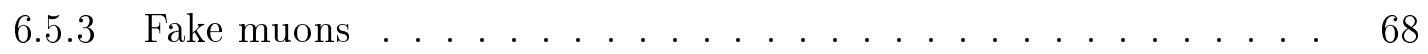

6.6 Continuum Background . . . . . . . . . . . . . . . . . . . . 69

6.7 Combinatoric Background . . . . . . . . . . . . . . 69

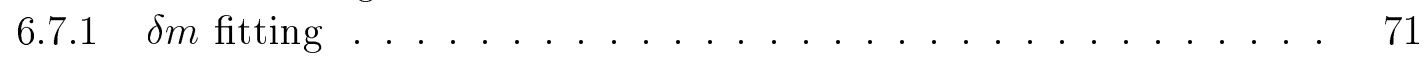

7 Branching Fraction Extraction . . . . . . . . . . . . . . 75

7.1 Fitting Signal and Background . . . . . . . . . . . . 75

7.1.1 Maximum Log Likelihood Function . . . . . . . . . . . . . . 75

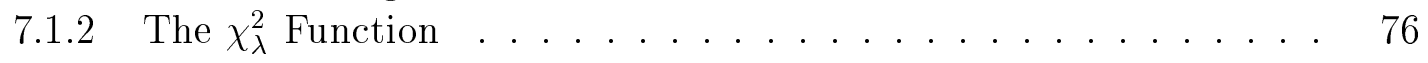

7.1 .3 The Input Histograms . . . . . . . . . . . . . . 76

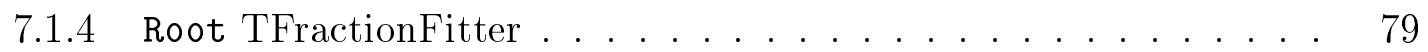


7.2 Fit Results . . . . . . . . . . . . . . . . . . . . 80

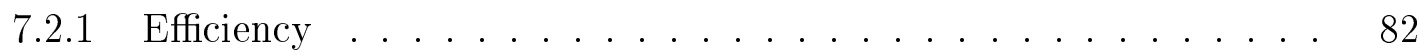

7.2.2 Calculating the Branching Fraction . . . . . . . . . 85

8 Validation and Systematic Studies . . . . . . . . . . . . . 87

8.1 Validation Studies . . . . . . . . . . . . . . . . . . . . . 87

8.1 .1 Spectra Comparisons . . . . . . . . . . . . . . . 87

8.1 .2 Vertexing Algorithm . . . . . . . . . . . . . . 90

8.1.3 Histogram Binning for $\mathcal{B}$ Fit . . . . . . . . . . . . . . . 91

8.1.4 Confirming the Fit Method . . . . . . . . . . . 91

$8.2 \mathrm{MC}$ and Data Statistics . . . . . . . . . . . . . . . . . 91

8.3 Systematic Errors from Analysis Method . . . . . . . . . . . . . . . 92

8.3.1 $D^{*+} \mu^{-} \bar{\nu}_{\mu} \pi$ Background . . . . . . . . . . . . . . . . 92

8.3.2 Muon Background Fractions . . . . . . . . . . . . . . . . 93

8.3.3 MC Truth Association . . . . . . . . . . . . . . . . . 94

8.3.4 Reconstruction Efficiency . . . . . . . . . . . . . . . . . 94

8.3.5 Continuum Background Representation . . . . . . . . . . . 94

8.4 BABAR Experiment Systematics . . . . . . . . . . . . . . . . . . . . . 95

8.4.1 PID Control Sample Statistics . . . . . . . . . . . . . . 95

8.4 .2 PID Corrections . . . . . . . . . . . . . . . . . . 96

8.4 .3 Tracking Efficiency . . . . . . . . . . . . . . . 97

8.4.4 Beam Energy Data/MC Disagreement . . . . . . . . . . 97

8.4.5 HQET Parameter Dependence . . . . . . . . . . . . . . . . 97

8.4.6 Number of $\Upsilon(4 S)$ Produced . . . . . . . . . . . . . . . . 98

8.5 Systematics from Externally Measured Branching Fractions . . . . . . . 98

8.6 Systematic Error Summary . . . . . . . . . . . . . . . . . 98

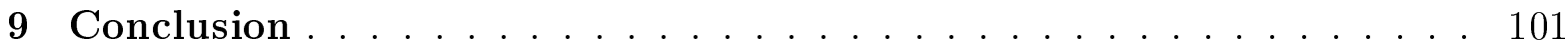

Bibliography . . . . . . . . . . . . . . . . . 103

Glossary . . . . . . . . . . . . . . . . . . . . . . . . . 109

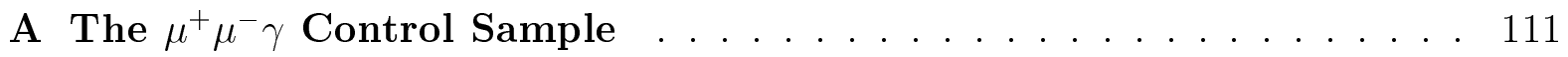

A.1 Introduction . . . . . . . . . . . . . . . . . . . . . . . . 111

A.2 Track Selection . . . . . . . . . . . . . . . 111

A.3 Purity . . . . . . . . . . . . . . . . . . . . . . . . . . . . . . . 112

A.4 Event Rate . . . . . . . . . . . . . . . . . . . . . 113

A.5 Monte Carlo Comparisons . . . . . . . . . . . . . . . . . . . 114

A.6 $\mu \mathrm{ID}$ Efficiencies . . . . . . . . . . . . . . . . . . . . . . . 114

A.7 Conclusion . . . . . . . . . . . . . . . . . . 115

B My Contribution to the BABAR Experiment . . . . . . . . . . 125

C Countries and Institutions in the BABAR collaboration . . . . . . . 127 
viii

Contents 


\section{List of Tables}

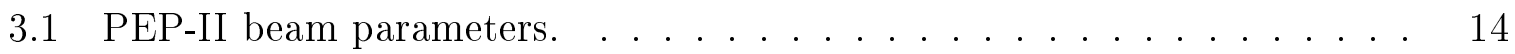

3.2 DCH wire specifications. . . . . . . . . . . . . . . 20

3.3 Cross sections for the principal physics processes at $10.58 \mathrm{GeV}$. . . . 33

4.1 Tracking list definitions . . . . . . . . . . . . . . . 40

4.2 Muon identification categories and criteria. . . . . . . . . . . . . . 41

4.3 Criteria of kaon identification selectors. . . . . . . . . . . . . . . 42

5.1 MC samples used in this analysis. . . . . . . . . . . . . . . 47

5.2 Parameters for slow pion efficiency function. . . . . . . . . . . 57

$6.1 B \rightarrow D^{*+} \ell^{-} \bar{\nu}_{\ell} \pi$ decay modes in generic MC. . . . . . . . . . . 64

$6.2 B$ meson decay modes contributing to the correlated muon background. . 66

6.3 Integrated yields in $\delta m$ peaking region. . . . . . . . . . . . 72

7.1 The fractions returned by the main fit. . . . . . . . . . . . . 80

7.2 Values entering final $\mathcal{B}$ calculation . . . . . . . . . . . 85

8.1 Effects of changing constraints in the vertexing algorithm. . . . . . . 90

8.2 Results of fitting uses different bin sizes. . . . . . . . . . . . . . . . . . . 91

8.3 MC and Data statistical errors on $\mathcal{B}$ value. . . . . . . . . . . . . . . . . 92

8.4 Systematic effects of $D^{*+} \mu^{-} \bar{\nu}_{\mu} \pi$ background samples. . . . . . . . . . . 93

8.5 Systematic effects of muon backgrounds. . . . . . . . . . . . . 94

8.6 Systematic effect in continuum background representation. . . . . . . 95

8.7 Average efficiencies for different control samples. . . . . . . . . . . . . . . . 96

8.8 Systematic effect of muon control sample. . . . . . . . . . . . . . . . 96

$8.9 \mathcal{B}$ shifts with HQET parameters. . . . . . . . . . . . . . 98

8.10 Values entering final $\mathcal{B}$ calculation . . . . . . . . . . . . . . . . . . . 98

8.11 Summary of systematic errors. . . . . . . . . . . . . . 99

A.1 Contamination in dilepton samples . . . . . . . . . . . 113 


\section{List of Figures}

1.1 Feynman diagram of the $\bar{B}^{0} \rightarrow D^{*+} \ell^{-} \bar{\nu}_{\ell}$ decay. . . . . . . . . . . . 2

1.2 Diagram of the particle interactions involved in this analysis. . . . . . . 3

2.1 Feynman diagram of a quark decay . . . . . . . . . . . . . 6

2.2 The unitarity triangle . . . . . . . . . . . . . . . 7

3.1 BABAR detector side view. . . . . . . . . . . . . . . . 11

3.2 BABAR detector end view. . . . . . . . . . . . . . . . 12

3.3 SLAC and PEP-II layout. . . . . . . . . . . . . . . . 14

3.4 BABAR interaction region . . . . . . . . . . . . . . 15

$3.5 \Upsilon(4 S)$ energy scan . . . . . . . . . . . . . . . . 16

3.6 Schematic end view of the SVT. . . . . . . . . . . 17

3.7 Side view of SVT . . . . . . . . . . . . . . . . 18

3.8 SVT hit resolution . . . . . . . . . . . . . . . . 19

3.9 Schematic view of DCH with principle dimensions. . . . . . . . . 20

3.10 Layout of DCH drift cells . . . . . . . . . . . . . . . . . . 21

3.11 DCH position resolution . . . . . . . . . . . . . . 22

3.12 Measurements of $\mathrm{d} E / \mathrm{d} x$ versus track momenta. . . . . . . . . . . . 23

3.13 Schematics of the DIRC fused silica bar and imaging region. . . . . . . . 24

3.14 View of DIRC system. . . . . . . . . . . . . . . 25

3.15 Cross section of the EMC . . . . . . . . . . . . 26

3.16 Diagram of an EMC crystal . . . . . . . . . . . . . . . . . . . 27

3.17 Magnetic field components $B_{z}$ and $B_{r} \ldots \ldots \ldots \ldots$. . . . . 29

3.18 Schematic view of the IFR . . . . . . . . . . . . . . 30

3.19 Cross section of a planar RPC . . . . . . . . . . . . . 31

3.20 Muon detection efficiency over time. . . . . . . . . . . . . . . 32

4.1 Track reconstruction efficiency in the DCH . . . . . . . . . 36

4.2 Tracking efficiency in the SVT . . . . . . . . . . . . . . . . . . 38

4.3 Track parameter resolutions based on cosmic ray muons. . . . . . . . . 39

4.4 Resolutions of parameters $d_{0}$ and $z_{0}$ vs momentum $\ldots \ldots$. . . . . 39

5.1 The decay chain reconstructed in this analysis. . . . . . . . . . 48

5.2 Raw $D^{0}$ candidate mass distribution. . . . . . . . . . . . . . . . . . . 49

$5.3 \chi^{2}$ probability of $D^{0}$ mass-constrained fit. . . . . . . . . . . . . . . 49

$5.4 D^{*+}$ momentum spectra in the CM frame. . . . . . . . . . . 50 
$5.5 \delta m$ plots for slow pion in SVT or SVT+DCH. . . . . . . . . . 51

5.6 Transverse momentum of slow pion. . . . . . . . . . . . . . 51

5.7 Lepton momentum spectrum in CM frame. . . . . . . . . . . . . . . . . . 53

$5.8 \cos \theta_{D^{*}, \ell}$ for signal and background leptons. . . . . . . . . . . . . 53

5.9 Distribution of thrust angle between $D^{*+} \ell^{-}$candidate and rest of event. . 55

$6.1 \cos \theta_{B, D^{*} \ell}$ for $\bar{B}^{0} \rightarrow D^{*+} \mu^{-} \bar{\nu}_{\mu}$ signal events. . . . . . . . . . . . . . 62

6.2 Pictorial view of the $D$ meson. . . . . . . . . . . . . . . . . 63

$6.3 \cos \theta_{B, D^{*} \ell}$ for $B \rightarrow D^{*+} \mu^{-} \bar{\nu}_{\mu} \pi$ background. . . . . . . . . . . . 66

$6.4 \cos \theta_{B, D^{*} \ell}$ for correlated muon background. . . . . . . . . . . . . . 67

$6.5 \cos \theta_{B, D^{*} \ell}$ for uncorrelated muon background. . . . . . . . . . . . 68

$6.6 \cos \theta_{B, D^{*} \ell}$ for fake muon background. . . . . . . . . . . . . . . . . . . . . . . . . . 69

$6.7 \cos \theta_{B, D^{*} \ell}$ for continuum background. . . . . . . . . . . . . . . 70

$6.8 \cos \theta_{B, D^{*} \ell}$ for combinatoric background. . . . . . . . . . . . . 70

6.9 Fits to the $\delta m$ plot. . . . . . . . . . . . . . . 71

$7.1 \cos \theta_{B, D^{*} \ell}$ for combined lepton backgrounds. . . . . . . . . . 77

$7.2 \cos \theta_{B, D^{*} \ell}$ inputs to main fit routine. . . . . . . . . . . . . . . . . . . . . . . . . . . . . .

7.3 Results from the fit. . . . . . . . . . . . . . . . 81

7.4 Comparing the predicted templates with the input templates. . . . . 83

7.5 Pulls for the fitted results. . . . . . . . . . . . . . 84

8.1 Comparisons of muon spectra for data and $\mathrm{MC} \ldots \ldots$. . . . . . . 88

8.2 Comparisons of slow $\pi$ spectra for data and MC . . . . . . . . . . 89

8.3 Comparison of $p_{D^{*}}^{*}$ for data and MC . . . . . . . . . . . 90

9.1 Comparison to other $\mathcal{B}\left(\bar{B}^{0} \rightarrow D^{*+} \mu^{-} \bar{\nu}_{\mu}\right)$ measurements. . . . . . . . . 102

A.1 Momentum vs $\theta$ of unbiased muon tracks from data and MC. . . . . . 113

A.2 Spectra of unbiased muon tracks in data and MC. . . . . . . . . . . . 114

A.3 $\mu$ ID efficiencies for each selector calculated from the $\mu \mu \gamma$ sample. . . . 116

A.4 Absolute efficiency differences between the $\mu \mu \gamma$ and $e e \mu \mu$ control samples. 117

A.5 Number of entries per bin in the $\mu \mu \gamma$ sample. . . . . . . . . . . . . 118

A.6 Absolute efficiency differences $(\mu \mu \gamma-e e \mu \mu)$ for MIP selector. . . . . . 119

A.7 Absolute efficiency differences $(\mu \mu \gamma-e e \mu \mu)$ for Very Loose selector. . . . 120

A.8 Absolute efficiency differences $(\mu \mu \gamma-e e \mu \mu)$ for Loose selector. . . . . . . 121

A.9 Absolute efficiency differences $(\mu \mu \gamma-e e \mu \mu)$ for Tight selector. . . . . . 122

A.10 Absolute efficiency differences $(\mu \mu \gamma-e e \mu \mu)$ for Very Tight selector. . . . 123 
For my wife Lori, who has sacrificed more than I have in order to make this thesis possible. 
xiv 


\section{Acknowledgements}

First and foremost, I want to thank my wife, Lori who has put off her own education, career, and potential family for so long in order to give me the time to complete my education. While her faith in me wavered at times, I am very grateful that she stuck by me and supported me till the end. Now it's my turn.

My thesis supervisor, Janis McKenna has given me enormous support on many different levels. She has been mentor, confidant, enthusiast, and a shining example of what an adviser can give to her students. It has been a privilege being her student.

Many thanks to Tom Mattison and Chris Hearty for sharing their knowledge and insight in helping me to stay on track and move forward in my analysis.

To Javed Iqbal \& Jess Brewer, on my supervisory committee for providing excellent feedback on my thesis.

To Rolf Dubitzky, Cristiano Borean, Chih-hsiang Cheng, Tim Meyer, and the rest of the BABAR $D^{*+} \ell^{-} \bar{\nu}_{\ell}$ group for providing much of the ground work for this analysis, and for answering my many questions along the way.

My thanks go out to my parents and to Lori's parents for providing financial and emotional support to both of us to keep us going over the years.

To Doug Maas, our computer and engineering technician. How could I get anything done without him keeping the computers in our lab running well?

To Steve Sekula and Andrew Eichenbaum for Settlers and surrealism during my visits to SLAC.

To Pat \& Jess Norman for making their home feel like a second home to me.

To my fellow UBC BABAR students over the years - Dave, Nasim, Song, Bryan, Zerah $\&$ Stephen to name a few - for friendship, feedback and helping to make the lab a great place to work. 


\section{Chapter 1}

\section{Introduction}

The twentieth century marked a period of enormous growth in the field of particle physics. Throughout the century, newer and more exotic particles were discovered, which naturally led to the question of how they interact. A whole new picture of the world emerged to explain these remarkable discoveries: the Standard Model of particle physics.

The Standard Model describes the framework in which the fundamental particles, quarks and leptons, interact with each other through the exchange of force-carrying bosons. It incorporates the theories of quantum mechanics and special relativity; both of which play a vital role in particle physics. The scope of the Standard Model is very impressive. It has implications in Big Bang cosmology, radiation cancer therapy, and quantum computing. It is believed that if the Standard Model could be united with the theory of general relativity (possibly through String Theory), the result would be the Grand Unified Theory of Everything.

The Standard Model is based on 19 fundamental parameters. Yet, it has successfully predicted results from thousands of experiments and observations. One of the important aspects of the Standard Model is the set of parameters describing quark decay. This is described by the Cabibbo-Kobayashi-Maskawa $(\mathrm{CKM})^{\mathrm{a}}$ matrix, ${ }^{[1,2]}$ which shows the coupling of the quark weak eigenstates to their mass eigenstates:

$$
\left[\begin{array}{l}
d^{\prime} \\
s^{\prime} \\
b^{\prime}
\end{array}\right]=\left[\begin{array}{lll}
V_{u d} & V_{u s} & V_{u b} \\
V_{c d} & V_{c s} & V_{c b} \\
V_{t d} & V_{t s} & V_{t b}
\end{array}\right]\left[\begin{array}{l}
d \\
s \\
b
\end{array}\right]
$$

The CKM matrix plays a very important role in the Standard Model. It is critical to our understanding of the weak interactions and $\mathrm{CP}$ violation. If the matrix elements can be measured with enough precision, the matrix could indicate aspects of physics beyond the Standard Model. ${ }^{[3-5]}$

The elements that make up the CKM matrix are fundamental parameters of the Standard Model. However, only a few of the matrix elements are very well measured. Those involving the bottom and top quarks are particularly difficult to measure because of the limit in the amount of data available, and also the uncertainties in the theoretical models used to extract the elements from experimental observables. Currently, the $90 \%$ confidence limits on the magnitudes of the matrix elements are ${ }^{[6]}$

$$
V_{C K M}=\left[\begin{array}{ccc}
0.9741-0.9756 & 0.219-0.226 & 0.0025-0.0048 \\
0.219-0.226 & 0.9732-0.9748 & 0.038-0.044 \\
0.004-0.014 & 0.037-0.044 & 0.9990-0.9993
\end{array}\right]
$$

\footnotetext{
${ }^{\text {a }} \mathrm{A}$ glossary is located at the end of this thesis to define the acronyms and abbreviations used herein.
} 


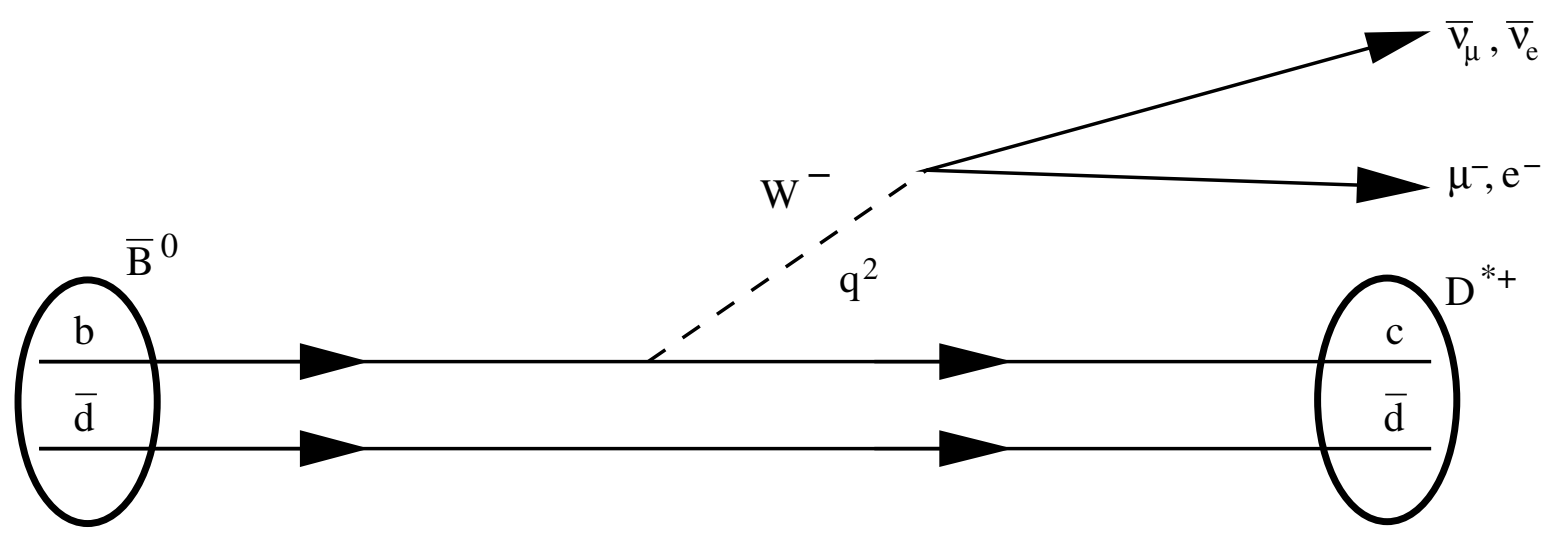

Figure 1.1: Feynman diagram of the $\bar{B}^{0} \rightarrow D^{*+} \ell^{-} \bar{\nu}_{\ell}$ decay. The virtual $W$ boson carries 4-momentum $q$.

Improved knowledge of these values will provide greater predictive ability in the Standard Model, and allow for refinements to be made with better accuracy.

The BABAR experiment was designed to provide a very detailed study of bottom quark physics. It has been collecting $B$ meson data since 1999 in order to study many of the aspects of $B$ meson ( $b$ quark) decay; one of which is the precision measurement of the matrix element $\left|V_{c b}\right|$. This can be studied using many different $b \rightarrow c$ quark decay channels. This thesis focuses on the exclusive semileptonic decay $\bar{B}^{0} \rightarrow D^{*+} \ell^{-} \bar{\nu}_{\ell}$, where the bottom quark decays into a charm quark with the release of two leptons, as shown in Figure 1.1. ${ }^{\mathrm{b}}$ The leptons considered in this analysis are either a muon or an electron, and the corresponding neutrino. The semileptonic decay provides a very clean signal for studying $\left|V_{c b}\right|$ because the only quarks involved in the interaction are the $b$ and $c$ quarks. The $d$ quark in the meson acts only as a spectator to the decay. If the virtual $\mathrm{W}$ produced a quark-antiquark pair instead of leptons, these other quarks would complicate the extraction of the signal.

BABAR takes place at the Stanford Linear Accelerator Center (SLAC) where electrons and positrons are collided at a center-of-mass energy of $10.58 \mathrm{GeV}$ to produce the $\Upsilon(4 S)$ resonance. The basic particle interactions involved in this analysis are shown in Figure 1.2. The events being analyzed are required to have the following decay chain. The $\Upsilon(4 S)$ immediately decays into a pair of $B^{0} \bar{B}^{0}$ mesons. The $\bar{B}^{0}$ then decays into $D^{*+} \mu^{-} \bar{\nu}_{\mu}$. The $D^{*+}$ decays further into $D^{0} \pi^{+}$, and the $D^{0}$ decays into $K^{-} \pi^{+}$. Multiplying the branching fractions shown in Figure 1.2, and accounting for both $B^{0}$ mesons in the event, shows that for every million $\Upsilon(4 S)$ mesons produced, there will be approximately 1300 events of interest to this analysis.

$D^{*+}$ is an $S=1, L=0, J=1$ excited state of the $D$ particle. While $\left|V_{c b}\right|$ can be measured using the ground state, there are several advantages to using the $D^{*+}$ decay. First, the branching fraction for $\bar{B}^{0} \rightarrow D^{*+} \ell^{-} \bar{\nu}_{\ell}$ is almost three times as large as the

\footnotetext{
${ }^{\mathrm{b}}$ By convention, the $\bar{B}^{0}$ meson contains the $b$ quark, while the charged conjugate $B^{0}$ contains the antiquark $\bar{b}$. Throughout this thesis, when a decay mode is specified, the charge conjugate mode is also included, unless explicitly stated otherwise.
} 


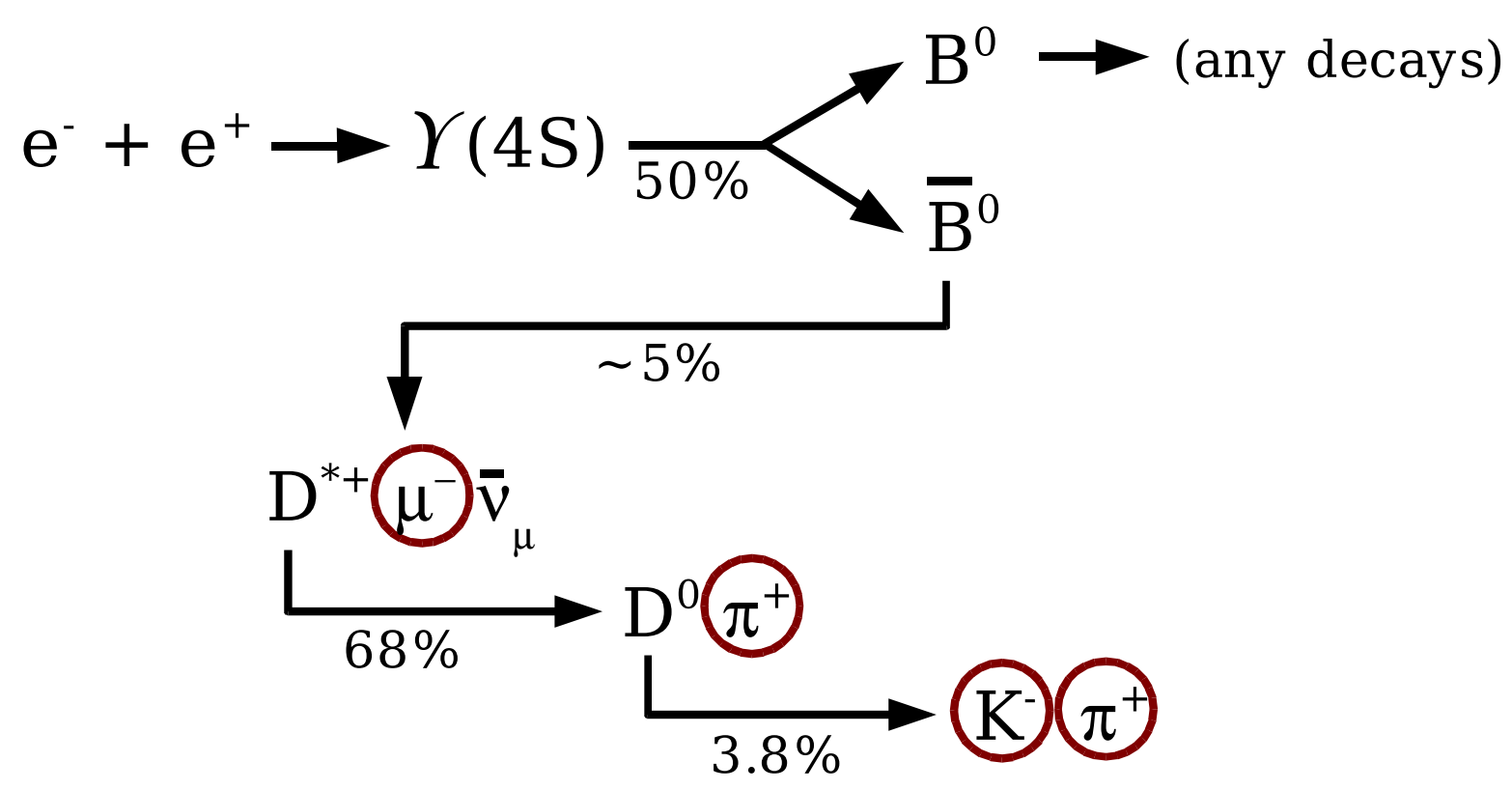

Figure 1.2: Diagram of the particle interactions involved in this analysis. At each interaction, there are numerous possible outcomes. The approximate branching fractions for the decays shown are given along the arrows. The circled particles indicate the final state particles observed by the BABAR detector. The decay of the other $B$ meson in the event also produces detectable particles which contribute to the backgrounds in the analysis.

branching fraction for $\bar{B}^{0} \rightarrow D^{+} \ell^{-} \bar{\nu}_{\ell}{ }^{[7]}$ Also, the decay of $D^{*+} \rightarrow D^{+} \pi^{0}$ would add considerable background to the $\bar{B}^{0} \rightarrow D^{+} \ell^{-} \bar{\nu}_{\ell}$ signal. Even more important is the small mass difference between the $m_{D^{*}}$ and $m_{D^{0}}$ which results in a very small background in the $D^{*+}$ sample due to the small phase space in the $D^{*+} \rightarrow D^{0} \pi^{+}$decay.

There is an additional advantage which reduces the error in the theoretical modeling of the decay. The form factors for the decays are calculated perturbatively in orders of $\left(1 / m_{q}\right)^{n}$. In the case where the $B$ decays to a $D^{*}$ which remains at rest in the $B$ frame, the leading order correction term $\left(1 / m_{q}\right)$ becomes zero. This is known as Luke's Theorem. ${ }^{[8]}$ This same condition is not true for the $\bar{B}^{0} \rightarrow D^{+} \ell \bar{\nu}_{\ell}$ decay. ${ }^{[9]}$

The procedure for measuring $\left|V_{c b}\right|$ from the $\bar{B}^{0} \rightarrow D^{*+} \ell^{-} \bar{\nu}_{\ell}$ decay involves first measuring the branching fraction. It is only through an accurate measurement of the branching fraction that the value of $\left|V_{c b}\right|$ can be extracted. The process for this extraction is described in the next chapter.

This thesis details the measurement of the branching fraction for the decay $\bar{B}^{0} \rightarrow$ $D^{*+} \mu^{-} \bar{\nu}_{\mu}$. The electron channel and the measurement of $\left|V_{c b}\right|$ are currently being studied by complementary analyses within BABAR. ${ }^{[10,11]}$ Given the strong model dependence in the determination on $\left|V_{c b}\right|$, it is important that the branching fraction is well measured. This analysis provides a valuable cross check to the branching fraction being used in the $B A B A R$ measurement of $\left|V_{c b}\right|$.

In addition to $\left|V_{c b}\right|$, the $\bar{B}^{0} \rightarrow D^{*+} \ell^{-} \bar{\nu}_{\ell}$ branching fraction is also important for 
many other studies. The $\bar{B}^{0} \rightarrow D^{*+} \ell^{-} \bar{\nu}_{\ell}$ decay is one of the highest probability $B^{0}$ decays, and it produces a very clean signal. It is used to study $B^{0}$ mixing and lifetimes, charm production rates from $B^{0}$ decays, and other properties. A precise knowledge of the $\bar{B}^{0} \rightarrow D^{*+} \ell^{-} \bar{\nu}_{\ell}$ branching fraction will improve the precision of many different measurements.

The thesis is structured in the following way. Chapter 2 describes the basic physics theory behind the measurement of the branching fraction and $\left|V_{c b}\right|$. Chapter 3 describes the $B A B A R$ experiment and detector. The analysis of raw data to identify tracks and particle species is described in Chapter 4. Chapters 5 and 6 detail the selection of data and Monte Carlo simulated events, and the cuts and techniques used to produce a clean signal of the $B^{0} \rightarrow D^{*-} \mu^{+} \nu_{\mu}$ decay. The fitting technique used to extract the number of decay events in the data sample, and the calculations to determine the branching fraction are described in Chapter 7 . Chapter 8 describes the studies done to validate the analysis and calculate the systematic errors on the measurement. Finally, the conclusion is presented in Chapter 9. 


\section{Chapter 2}

\section{Theoretical Background}

\subsection{The Standard Model}

In the Standard Model, the particles that make up matter are the quarks and leptons. They interact via three fundamental forces by exchanging force-carrying bosons. The photon carries the electromagnetic force; the weak force is carried by the $Z^{0}$ and $W^{ \pm}$ bosons, and the strong force is carried by the gluons. The particles are represented mathematically as fields in space-time, and the interaction as a Lagrangian function of the fields and their first derivatives.

One of the very important aspects of the Standard Model is its underlying symmetries. A symmetry in a physical system is any type of transformation applied to the Lagrangian which leaves it invariant. Noether's Theorem ${ }^{[12]}$ states that for a system described by a Lagrangian, any symmetry which leaves the action invariant implies the existence of a conservation law. There are many types of symmetries which can be seen in nature. For example, translations in space and time which leave the system invariant lead to conservation of momentum and energy respectively. These transformations can be varying at different space-time points (local) or global. Other types include gauge symmetries, and the discrete symmetries of parity, charge conjugation, and time reversal.

The Standard Model is based on $S U(3) \times S U(2) \times U(1)$ gauge symmetries. The $U(1)$ group is a one-dimensional phase rotation. $S U(2) \times U(1)$ are the symmetry groups governing the electroweak interactions, known as the Glashow-Weinberg-Salam model. ${ }^{[3-5]}$ $S U(3)$ is the symmetry group of the strong interaction, or quantum chromodynamics (QCD).

\section{$2.2 \quad C, P$, and $T$ Symmetry}

A very important set of symmetries in the Standard Model, or any field theory describing particle physics, are the discrete symmetries of:

- Charge conjugation $(C)$, where a particle is switched with its antiparticle,

- Parity $(P)$, where the space coordinates are reversed,

- Time reversal $(T)$, where the time coordinate is reversed.

While it is possible to predict violations of individual symmetries, the combined operation of $C P T$ is always symmetric in the Standard Model. ${ }^{[1]}$ 


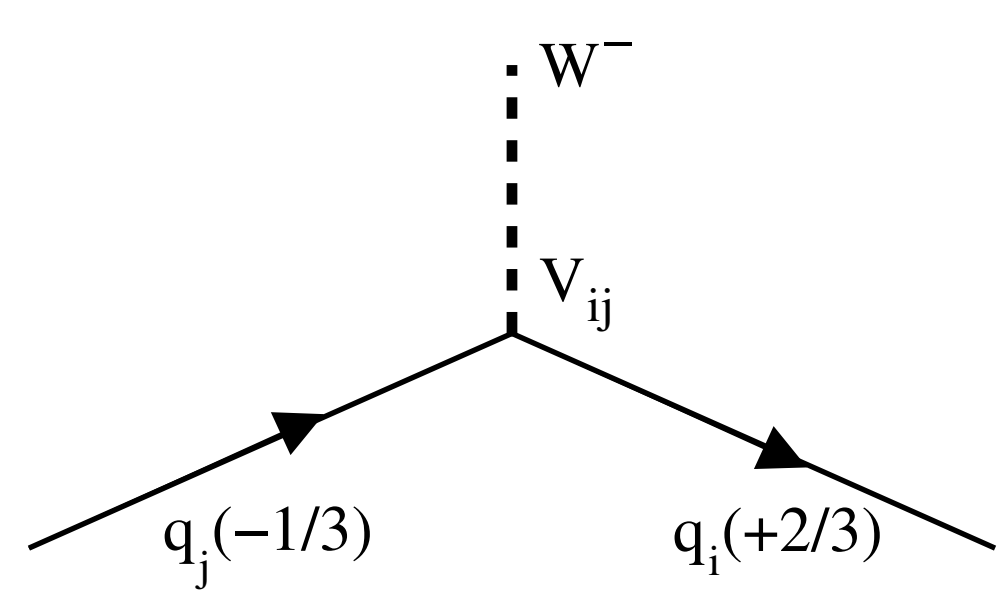

Figure 2.1: Feynman diagram of a quark of charge $-1 / 3$ decaying into a $+2 / 3$ charged quark through the release of a virtual $W$ boson.

While interactions governed by the strong and electromagnetic forces are invariant under $C, P, \& T$ separately, the weak interaction violates both $C$ and $P .{ }^{[15]}$ This is due to the $V-A$ (vector - axial vector) structure of the weak coupling. Yet, the combination of $C P$ together was thought for quite some time to be conserved. However, in 1964, $C P$ violation was seen in the neutral kaon system. ${ }^{[16]}$

Up until recently, the neutral kaon remained the only system in which any $C P$ violation was observed. The main design goal of the BABAR experiment was to measure $C P$ violation in the neutral $B$ system. Along with an analogous experiment, Belle, taking place at the KEK facility in Japan, BABAR published the first non-zero measurements of this in 2001. ${ }^{[17,18]} C P$ violation in the $B^{0}$ system is allowed in the Standard Model. This also leads to the prediction of $T$ violation when $C P$ is violated, in order to maintain overall $C P T$ invariance. At this time, no measurements of $T$ violation have yielded any non-zero results.

\subsection{The CKM Matrix}

The amplitude describing the quark decay through the flavour-changing charged current (Figure 2.1) is

$$
\mathcal{A}=i\left(\frac{G_{F} M_{W}^{2}}{\sqrt{2}}\right) V_{i j} \bar{q}_{i} \gamma_{\mu}\left(1-\gamma_{5}\right) q_{j}
$$

where $G_{F}$ is the Fermi coupling constant, $M_{W}$ is the mass of the $W$ boson, and $V_{i j}$ is from the Cabibbo-Kobayashi-Maskawa (CKM) matrix described in Eq. 1.1.

In the Standard Model, this matrix is unitary. Because of this, the nine parameters ${ }^{\mathrm{a}}$ in the matrix can be reduced to only four independent parameters: three real numbers

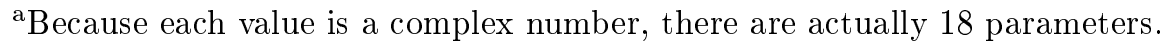




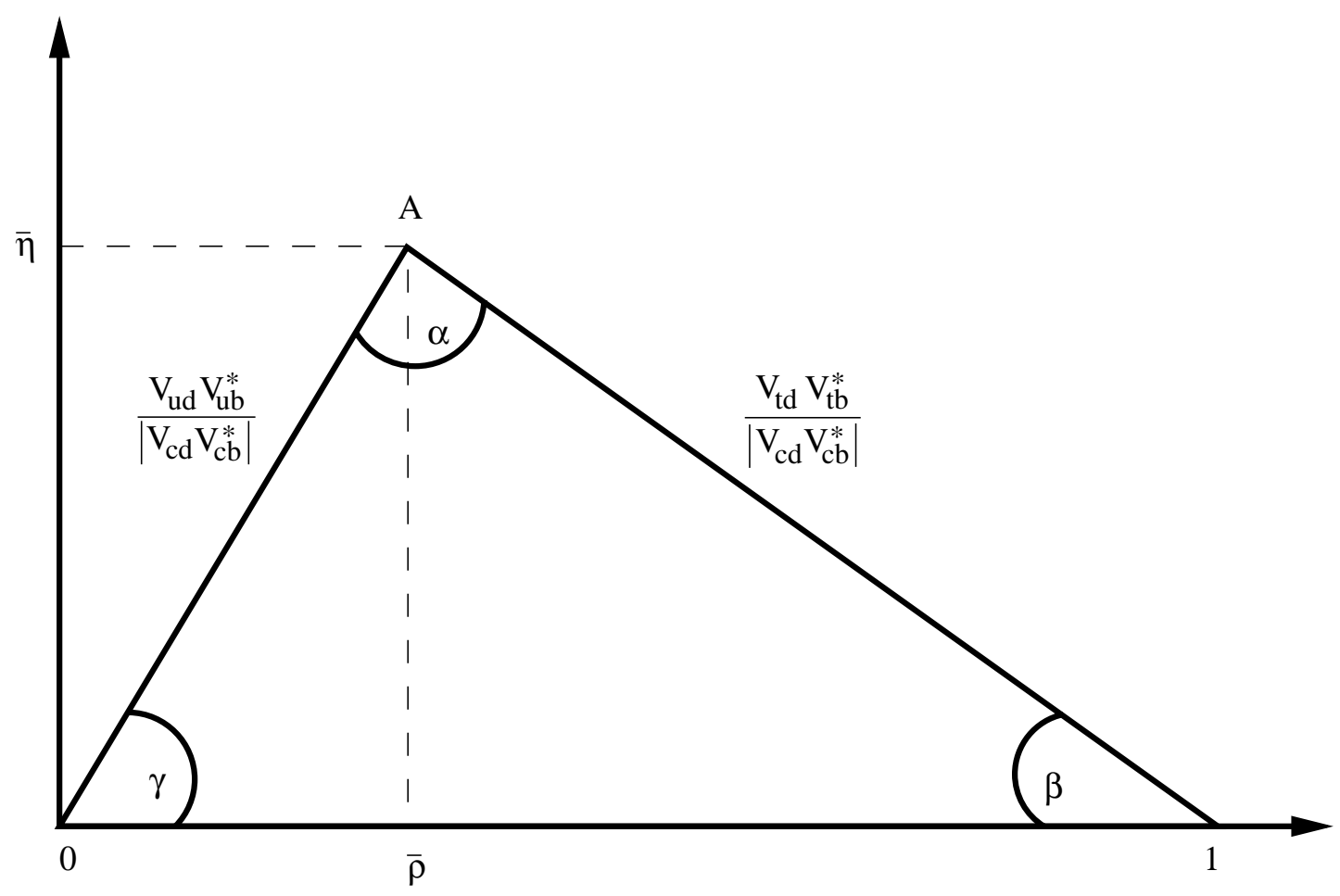

Figure 2.2: The unitarity triangle of the CKM matrix, normalized to 1 along the base. The top point of the triangle corresponds to $\bar{\rho}=\left(1-\lambda^{2} / 2\right) \rho$ and $\bar{\eta}=$ $\left(1-\lambda^{2} / 2\right) \eta$.

and a complex phase. It is the complex parameter which leads to the possibility of $C P$ violation.

A standard form for the matrix is the Wolfenstein ${ }^{[19]}$ parameterization. This is a power series expansion in terms of the parameter $\lambda=\sin \theta_{C} \sim 0.22$, where $\theta_{C}$ is called the Cabibbo angle. ${ }^{[1]}$ To order $\mathcal{O}\left(\lambda^{3}\right)$, it is expressed as

$$
V_{C K M}=\left(\begin{array}{ccc}
1-\frac{\lambda^{2}}{2} & \lambda & A \lambda^{3}(\rho-i \eta) \\
-\lambda & 1-\frac{\lambda^{2}}{2} & A \lambda^{2} \\
A \lambda^{3}(1-\rho-i \eta) & -A \lambda^{2} & 1
\end{array}\right)+\mathcal{O}\left(\lambda^{4}\right) .
$$

Since the matrix is unitary, the products of elements in any two columns satisfy

$$
\sum_{k=1}^{3} V_{k i} V_{k j}^{*}=\delta_{i j}
$$

where $\delta_{i j}=1$ if $i=j$, and $\delta_{i j}=0$ otherwise. For two different columns $(i \neq j)$, we can write Eq. (2.3) in terms of the matrix elements:

$$
\begin{aligned}
& V_{u d} V_{u s}^{*}\left[\mathcal{O}\left(\lambda^{1}\right)\right]+V_{c d} V_{c s}^{*}\left[\mathcal{O}\left(\lambda^{1}\right)\right]+V_{t d} V_{t s}^{*}\left[\mathcal{O}\left(\lambda^{5}\right)\right]=0 \\
& V_{u s} V_{u b}^{*}\left[\mathcal{O}\left(\lambda^{4}\right)\right]+V_{c s} V_{c b}^{*}\left[\mathcal{O}\left(\lambda^{2}\right)\right]+V_{t s} V_{t b}^{*}\left[\mathcal{O}\left(\lambda^{2}\right)\right]=0
\end{aligned}
$$




$$
V_{u d} V_{u b}^{*}\left[\mathcal{O}\left(\lambda^{3}\right)\right]+V_{c d} V_{c b}^{*}\left[\mathcal{O}\left(\lambda^{3}\right)\right]+V_{t d} V_{t b}^{*}\left[\mathcal{O}\left(\lambda^{3}\right)\right]=0,
$$

where $\mathcal{O}\left(\lambda^{n}\right)$ denotes the order of the leading $\lambda$ term.

These can be visualized as triangles in the complex plane. Two of these have a pair of long sides, and a much shorter side, so they would appear as very long, flat triangles. In Eq. (2.4a), the ratio between the long sides and the short is $\mathcal{O}\left(\lambda^{4}\right)$, and $\mathcal{O}\left(\lambda^{2}\right)$ for Eq. (2.4b). A much more even triangle is obtained from Eq. (2.4c), where all three sides are of $\mathcal{O}\left(\lambda^{3}\right)$; this is shown in Figure 2.2. In general, while all three triangles ${ }^{\mathrm{b}}$ represent the unitarity condition, the term unitarity triangle typically refers only to the third one.

The prime purpose of the BABAR experiment is to measure the different parameters of the unitarity triangle. The size of the $C P$ violating phase is simply the height of the triangle. However, experimental measurements involving $B$ decays can more directly probe the angles $(\alpha, \beta, \gamma)$. Accurate knowledge of these angles can define the position of the apex and therefore the value of the $C P$ violating phase. If this value proves to be outside the range predicted by the Standard Model, it could lead to the discovery of new physics beyond our current theory. Much of the effort in physics analysis at BABAR is in the study of $B$ meson decays into various $C P$ eigenstates which can yield values for these angles. However, this thesis does not focus on these types of decays. Instead, by studying the branching fraction for the decay $B^{0} \rightarrow D^{*-} \mu^{+} \nu_{\mu}$, one can obtain a measurement of the magnitude of the matrix element $\left|V_{c b}\right|$.

\subsection{Heavy Quark Effective Theory (HQET)}

In studying the $B$ meson decay, one can take advantage of the fact that the $b$ quark is a heavy quark. The size of the hadron is determined by the QCD length scale:

$$
R_{\text {had }} \sim 1 / \Lambda_{\mathrm{QCD}} \sim 1 \mathrm{fm} .
$$

The typical momenta exchanged in strong interactions between the heavy and light quark are of the order of $\Lambda_{\mathrm{QCD}} \simeq 0.2 \mathrm{GeV}$. Since the mass of the heavy quark $m_{Q} \simeq 5 \mathrm{GeV} \gg$ $\Lambda_{\mathrm{QCD}}$, the Compton wavelength of the heavy quark, $\lambda_{Q} \sim 1 / m_{Q}$ is much smaller than the scale of the hadron. The soft gluons exchanged between the two constituent quarks can only resolve distances much larger than $\lambda_{Q}$. So the meson becomes analogous to the hydrogen atom, with the light quark acting as the orbiting electron, whose wave function is decoupled from that of the heavy proton. ${ }^{[20]}$ The light quark becomes blind to the flavour of the quark acting as the nucleus, provided that it is heavy, i.e. $m_{Q} \rightarrow \infty$. The light quark also becomes blind to the spin of the heavy quark since spin decouples like $1 / m_{Q}$. It experiences only the colour field from the heavy quark.

The semileptonic decay $\bar{B}^{0} \rightarrow D^{*+} \ell^{-} \bar{\nu}_{\ell}$ is the classic example where HQET can be applied. The basic matrix element of the decay as shown in Figure 1.1 is given by

$$
-i \mathcal{M}=-i \frac{G_{F}}{\sqrt{2}} V_{c b} L_{\mu} H^{\mu}
$$

\footnotetext{
${ }^{\mathrm{b}}$ In fact, more triangles can be formed from the products of different rows of the matrix, but they are complex conjugates of the same equations.
} 
where $L_{\mu}$ is the lepton tensor

$$
L_{\mu}=\left\langle\ell\left|\gamma_{\mu}\left(1-\gamma_{5}\right)\right| \nu\right\rangle
$$

which is exactly calculable. The hadronic tensor, $H^{\mu}$, is generally composed of four form factors: $[24,25]$

$$
\begin{aligned}
H^{\mu}= & \left\langle D^{*+}(k, \epsilon)\left|\bar{c} \gamma^{\mu}\left(1-\gamma_{5}\right) b\right| \bar{B}^{0}(p)\right\rangle \\
= & {\left[\frac{2 i \epsilon^{\mu \nu \alpha \beta}}{m_{B}+m_{D^{*}}} \epsilon_{\nu}^{*} k_{\alpha} p_{\beta}\right] V\left(q^{2}\right)-\left[\left(m_{B}+m_{D^{*}}\right) \epsilon^{* \mu}\right] A_{1}\left(q^{2}\right)+} \\
& {\left[\frac{\epsilon^{*} \cdot p}{m_{B}+m_{D^{*}}}(p+k)^{\mu}\right] A_{2}\left(q^{2}\right)-\left[\frac{\epsilon^{*} \cdot p}{m_{B}+m_{D^{*}}}(p-k)^{\mu}\right] A_{3}\left(q^{2}\right) }
\end{aligned}
$$

where $V$ and $A$ stand for the underlying vector and axial-vector couplings, $p^{\mu}$ is the $\bar{B}^{0}$ momentum, $\epsilon^{\alpha}$ and $k^{\nu}$ are the $D^{*}$ polarization and momentum, and $q$ is the momentum carried by the $W$ :

$$
q^{2}=m_{B}^{2}+m_{D^{*}}^{2}-2 m_{B} m_{D^{*}}\left(v \cdot v^{\prime}\right)=m_{B}^{2}+m_{D^{*}}^{2}-2 m_{B} m_{D^{*}} w
$$

where $v$ and $v^{\prime}$ are the initial and final meson 4-velocities, and $w=v \cdot v^{\prime}$ is known as the recoil variable.

When the lepton mass is taken as negligible compared to the heavy quark masses, the $A_{3}$ form factor term becomes negligible, and the hadronic tensor can be parameterized ${ }^{[27]}$ in terms of the recoil variable by taking two form factor ratios:

$$
\begin{aligned}
& R_{1}(w)=\left[1-\frac{q^{2}}{\left(m_{B}+m_{D^{*}}\right)^{2}}\right] \frac{V\left(q^{2}\right)}{A_{1}\left(q^{2}\right)} \\
& R_{2}(w)=\left[1-\frac{q^{2}}{\left(m_{B}+m_{D^{*}}\right)^{2}}\right] \frac{A_{2}\left(q^{2}\right)}{A_{1}\left(q^{2}\right)},
\end{aligned}
$$

and adding an additional function, $\xi(w)$, known as the Isgur-Wise function. ${ }^{[20]}$ In the limit of $m_{q} \rightarrow \infty, R_{1}=R_{2} \rightarrow 1$, and the hadronic tensor reduces to a single function: $\xi(w)$.

In the heavy quark limit, the matrix element thus becomes

$$
\frac{1}{\sqrt{m_{B} m_{D^{*}}}}\left\langle D^{*+}\left(v^{\prime}\right)\left|\bar{c}_{v^{\prime}} \gamma^{\mu} b_{v}\right| \bar{B}^{0}(v)\right\rangle=\xi(w)\left(v+v^{\prime}\right)^{\mu}
$$

The differential decay rate can be obtained from the matrix element ${ }^{[26]}$ in terms of the recoil variable. 


$$
\begin{aligned}
\frac{\mathrm{d} \Gamma\left(\bar{B}^{0} \rightarrow D^{*+} \ell^{-} \bar{\nu}_{\ell}\right)}{\mathrm{d} w}= & \frac{G_{F}^{2}}{48 \pi^{3}} \mathcal{K}(w)\left|V_{c b}\right|^{2} \mathcal{F}^{2}(w) \\
= & \frac{G_{F}^{2}}{48 \pi^{3}}\left(m_{B}-m_{D *}\right)^{2} m_{D *}^{3} \sqrt{w^{2}-1}(w+1)^{2} \\
& \quad \times\left(1+\frac{4 w}{w+1} \frac{m_{B}^{2}-2 m_{B} m_{D *} w+m_{D *}^{2}}{\left(m_{B}-m_{D *}\right)^{2}}\right) \\
& \quad \times\left|V_{c b}\right|^{2} \mathcal{F}^{2}(w) .
\end{aligned}
$$

The function $\mathcal{K}(w)$ describes the phase space of the interaction, and the hadronic form factor $\mathcal{F}(w)$ represents the Isgur-Wise function with some corrections to account for the finite masses of the heavy quarks. The decay rate is therefore a function of $w$ and $\left|V_{c b}\right|^{2}$.

Unfortunately, even though the form factors have been reduced to only one function, $\mathcal{F}(w)$ is still not well defined. There are perturbative corrections of order $\left(\alpha_{s}\right)^{n}\left(m_{Q}\right)$ and of order $\left(\Lambda_{\mathrm{QCD}} / m_{Q}\right)^{n}$ where $m_{Q}$ can be either $m_{c}$ or $m_{b}$. While the $\alpha_{s}$ can be determined order by order in perturbation theory, this is not the case for the nonperturbative $\left(\Lambda_{\mathrm{QCD}} / m_{Q}\right)$ corrections. HQET can provide a good approximation to these corrections, but not an exact solution.

There is one place in the phase space at which $\mathcal{F}(w)$ can be well calculated: the zero-recoil point. This is where the $B$ decays to a $D^{*}$ which is at rest in the $B$ frame. This corresponds to the maximum momentum transfer to the virtual $\mathrm{W}$.

$$
\begin{aligned}
q_{\max }^{2} & =\left(m_{B}-m_{D^{*}}\right)^{2}, \quad \text { and } \\
w & =1 .
\end{aligned}
$$

Because the $D^{*}$ is at rest in the $B$ frame, the angular dependencies in the form factors drop out, and $\mathcal{F}(1)$ can be calculated with small and controlled theoretical errors. Since the range of $w$ varies only between $(1<w<1.5), \mathcal{F}(w)$ can be expanded around $w=1$ as a Taylor series

$$
\mathcal{F}(w)=\mathcal{F}(1)\left[1-\rho^{2}(w-1) \cdots\right] .
$$

The parameter $\rho^{2}$ is referred to as the slope of the form factor.

However, the phase space term becomes a problem at the zero recoil point: $\mathcal{K}(1)=0$. Thus, it is not possible to observe a decay occurring without some momentum transfer to the $D^{*}$. Therefore, the decay rate at $w=1$, where $\left|V_{c b}\right|$ could be extracted, cannot be measured directly. The strategy in measuring $\left|V_{c b}\right|$ is to measure the differential decay rate as a function of $w$, and extrapolate the data down to $w=1$. To do this requires a very good method for measuring the branching fraction in order to determine the differential decay rate. Once the overall branching fraction has been measured, the system can be investigated in bins of $w$, and the branching fraction measured in each bin in order to calculate the differential decay rate. By fitting a smooth curve to the plot of $\frac{\mathrm{d} \Gamma}{\mathrm{d} w} \mathrm{vs}$. $w$, the slope, $\rho^{2}$, and $\mathcal{F}^{2}(1)\left|V_{c b}\right|^{2}$ can be determined. 


\section{Chapter 3}

\section{The BABAR Experiment}

The data for this thesis were collected by the BABAR detector on the PEP-II asymmetric $B$ Factory at the Stanford Linear Accelerator Center (SLAC). The BABAR collaboration consists of approximately 600 physicists and engineers from 75 institutions in 10 countries. A full list of the participating countries and institutions is provided in Appendix C. This chapter will describe the SLAC/PEP-II accelerator and the BABAR detector. Information in this chapter is taken primarily from references $28-30$.

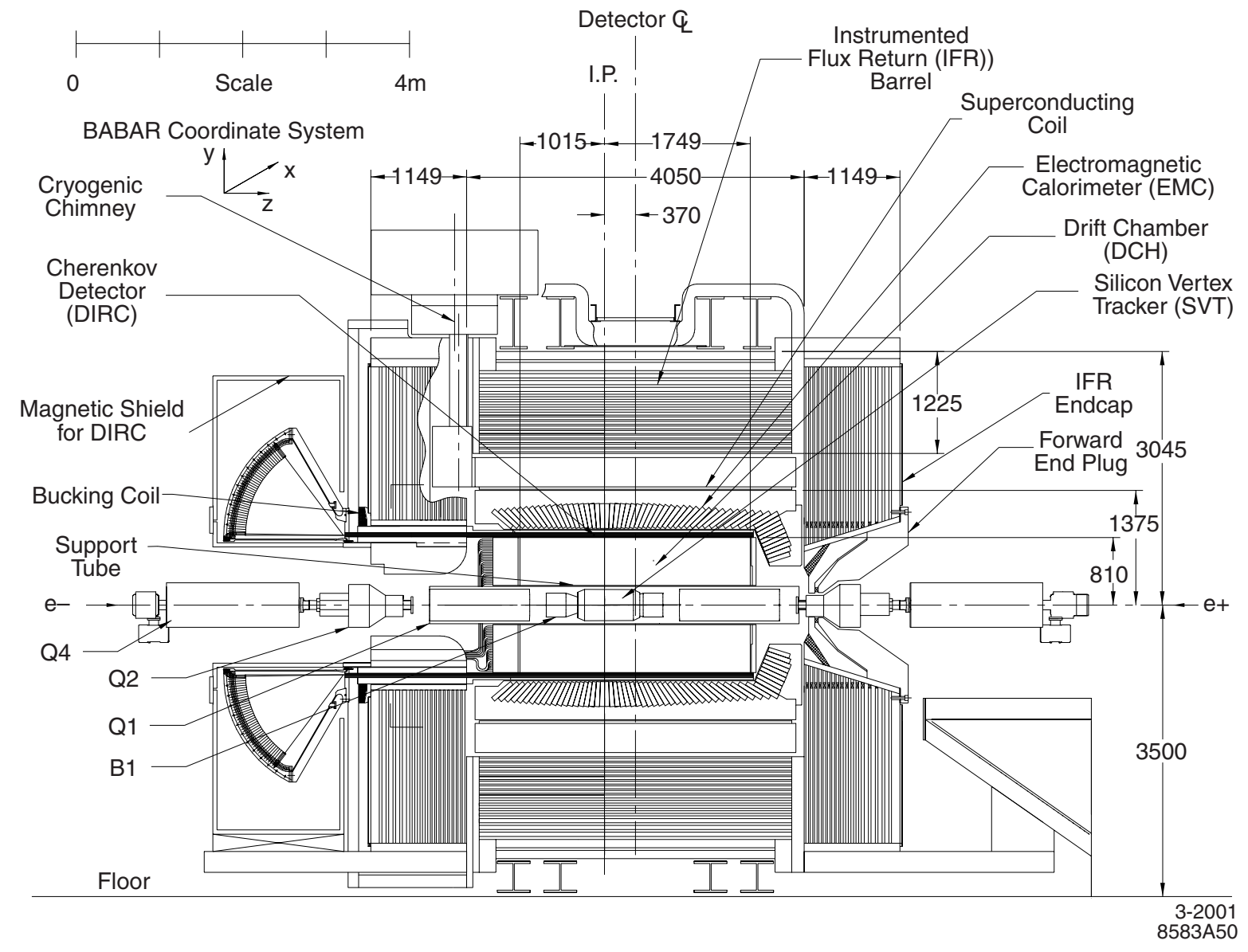

Figure 3.1: BABAR detector side view. 


\subsection{Overview}

The main goal of the BABAR experiment is the study of CP violation in neutral $B$ meson decays. Other goals include precision studies of decays of heavy mesons, $\tau$ leptons, and searches for rare decays. The design of the detector is optimized to meet these physics goals.

The PEP-II $B$ Factory is a high luminosity $e^{+} e^{-}$collider operating at the mass of the $\Upsilon(4 S)$ resonance: $10.58 \mathrm{GeV}$. This resonance decays exclusively to $B^{0} \bar{B}^{0}$ and $B^{+} B^{-}$with equal probability. The design calls for an instantaneous luminosity of $3 \times 10^{33} \mathrm{~cm}^{-2} \mathrm{~s}^{-1}$. This luminosity was achieved by the end of running in the year 2000 and exceeded in 2001. In PEP-II, 9.0 GeV electrons are collided with $3.1 \mathrm{GeV}$ positrons, resulting in a Lorentz boost to the $\Upsilon(4 S)$ resonance of $\beta \gamma=0.56$ in the laboratory frame. The boost is necessary for the reconstruction of separate decay vertices of the two $B$ mesons and thus the measurement of the time between the decays.

The branching fractions of $B$ mesons into CP eigenstates are of order $10^{-4}$ or less. In order to study the CP-violating asymmetries, one of the $B^{0}$ 's must be fully reconstructed from both charged and neutral final states, while the state of the other $B$ meson $\left(B^{0}\right.$ or $\bar{B}^{0}$ ) must be identifiable from its decay products. These requirements place stringent

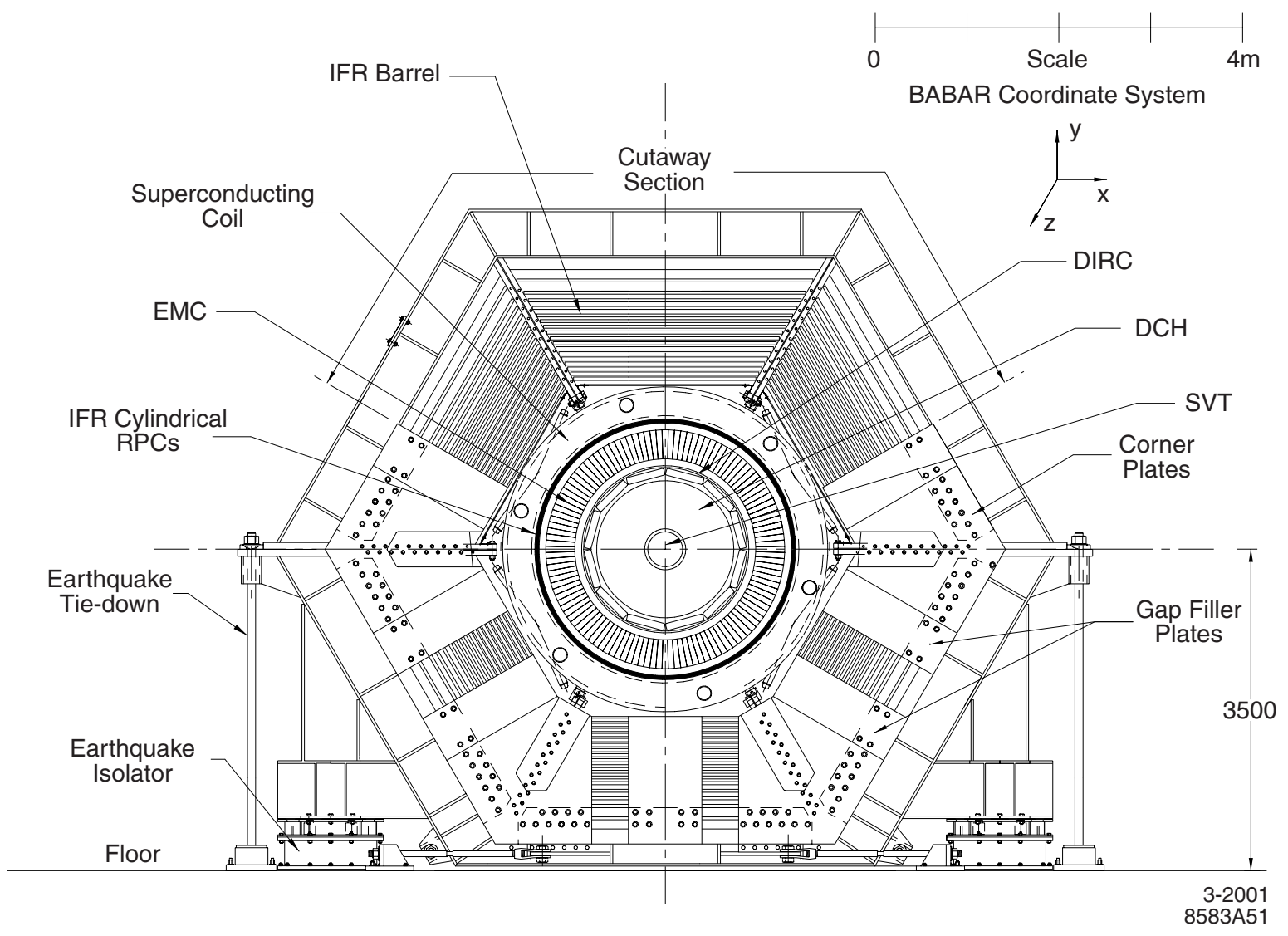

Figure 3.2: BABAR detector end view. 
criteria on the design of the detector.

It must have:

- a large acceptance down to small polar angles in the centre-of-mass (CM) frame.

- excellent reconstruction efficiency for charged particles and photons down to low energies.

- very good resolution in position, momentum, and energy.

- precise vertex resolution, both transverse and parallel to the beam direction.

- efficient particle identification (PID) for electrons, muons and kaons.

- a flexible and selective triggering system.

Figures 3.1 and 3.2 show schematic views of the BABAR detectors from the side and end. The inner part of the detector consists of a silicon vertex tracker (SVT) and a drift chamber $(\mathrm{DCH})$ for charged particle tracking and vertexing, a Cherenkov ring imaging detector (DIRC) for kaon identification, and an electromagnetic calorimeter (EMC) for measuring particle energies. Surrounding these systems is a superconducting solenoid which produces a $1.5 \mathrm{~T}$ axial magnetic field. Outside of the solenoid is the instrumented flux return (IFR) which is used for identifying muons and neutral hadrons. The coordinate system of the detector is right-handed and anchored on the principle tracking chamber, the DCH. The $z$-axis is along the principle axis of the detector, pointing in the direction of the electron beam. The $y$-axis points upward from the plane of the storage ring, and the $x$-axis points away from the centre of the PEP-II storage rings. The beam axis is offset relative to the $z$-axis by $20 \mathrm{mrad}$ in the horizontal plane.

The CM frame of the $\Upsilon(4 S)$ resonance is boosted in the direction of the electron beam. Because the CM frame is moving in the lab frame, the interaction point (IP) of the detector is shifted by $0.37 \mathrm{~m}$ upstream along the electron beam to provide an improved acceptance in the boost direction. The acceptance extends to $350 \mathrm{mrad}$ in the forward direction $(+z)$ and $400 \mathrm{mrad}$ in the backward direction, and is limited by dipole magnets on the PEP-II beam line for bending the beams into collision at the IP.

\section{$3.2 \quad$ PEP-II}

PEP-II is an $e^{+} e^{-}$storage ring operating at the centre-of-mass energy of $10.58 \mathrm{GeV}$ at high luminosity. It is $2200 \mathrm{~m}$ in circumference and located at the end of the SLAC linear accelerator, as shown in Figure 3.3. It consists of two storage rings: the high-energy electron ring (HER), and the low-energy positron ring (LER). The HER is built from the existing PEP (Positron Electron Project) beam line. The LER is situated on top of the HER, except within $\pm 60 \mathrm{~m}$ of the IP where the LER is brought into the same horizontal plane as the HER. The parameters of the electron and positron beams are shown in Table 3.1 . 


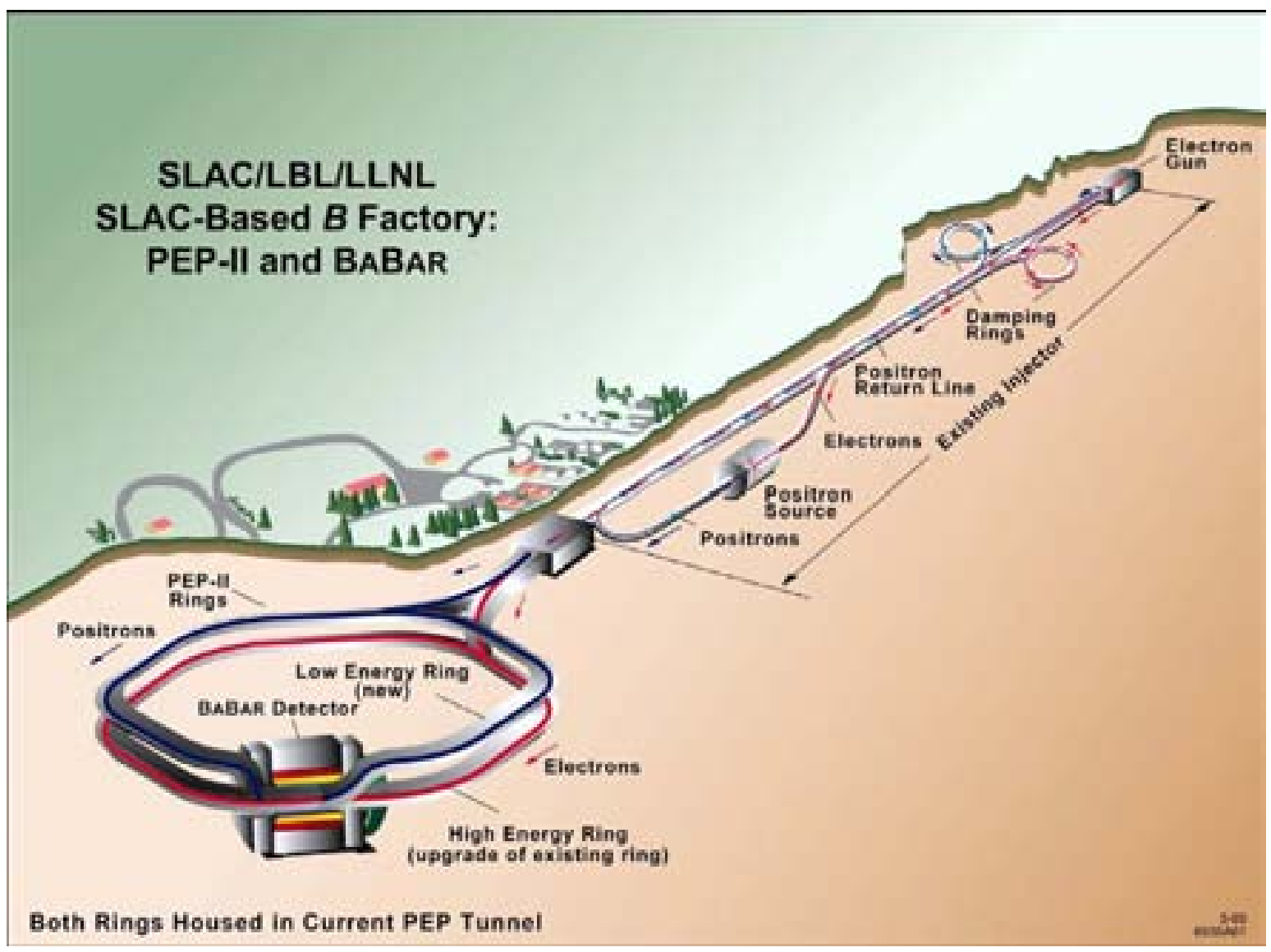

Figure 3.3: SLAC and PEP-II layout.

\begin{tabular}{|l|c|c|}
\hline Parameters & Design & Typical $(2000)$ \\
\hline Energy HER/LER $(\mathrm{GeV})$ & $9.0 / 3.1$ & $9.0 / 3.1$ \\
Current HER/LER (A) & $0.75 / 2.15$ & $0.7 / 1.3$ \\
\# of bunches & 1658 & $553-829$ \\
Bunch spacing $(\mathrm{ns})$ & 4.2 & $6.3-10.5$ \\
$\sigma_{L x}(\mu \mathrm{m})$ & 110 & 120 \\
$\sigma_{L y}(\mu \mathrm{m})$ & 3.3 & 5.6 \\
$\sigma_{L z}(\mathrm{~mm})$ & 9 & 9 \\
Luminosity $\left(10^{33} \mathrm{~cm}^{-2} \mathrm{~s}^{-1}\right)$ & 3 & 2.5 \\
Luminosity $\left(\mathrm{pb}^{-1} /\right.$ day $)$ & 135 & 120 \\
\hline
\end{tabular}

Table 3.1: PEP-II beam parameters. Values are given both for the design and for typical colliding beam operation in the first year. $\sigma_{L x}, \sigma_{L y}$, and $\sigma_{L z}$ refer to the horizontal, vertical, and longitudinal rms size of the luminous region.

The interaction region is the most challenging part of the PEP-II design. ${ }^{[31]}$ The zero crossing angle for the colliding beams requires a pair of bending magnets (B1) located at $\pm 21 \mathrm{~cm}$ from IP in order to avoid parasitic collisions. These magnets set the limits on the 


\section{Interaction Region}

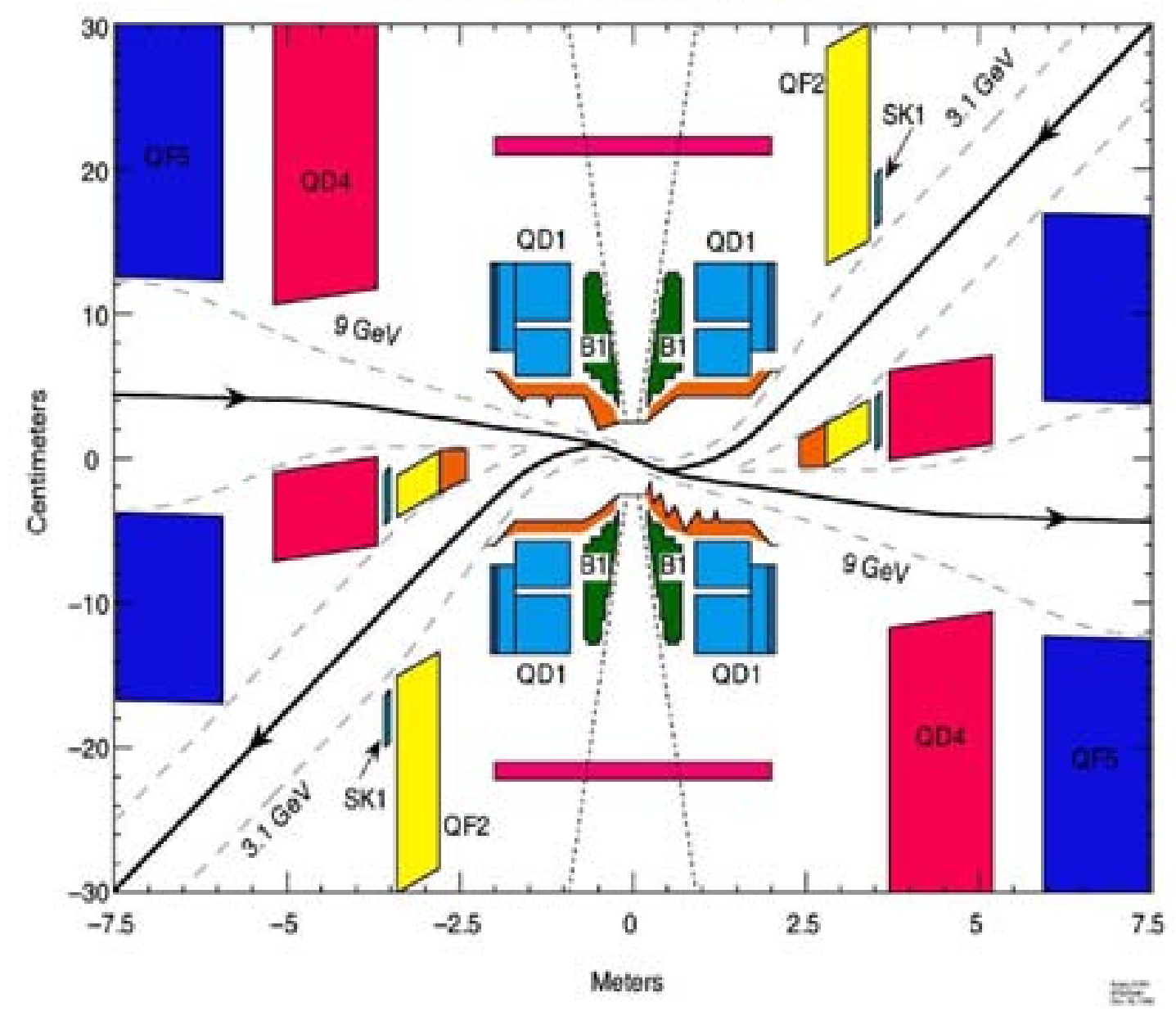

Figure 3.4: The BABAR interaction region. Shown are the final bending magnets (B1), focusing magnets, synchrotron radiation masks, and the HER/LER beam trajectories. Note the aspect ratio of the figure is not $1: 1$.

angular coverage of the detector. Additionally, beam-induced backgrounds are produced by synchrotron radiation from the final bending of the beams, and by electromagnetic showers generated in the extra material near the IP. This background needs to be properly shielded and monitored both to protect the detector components and to minimize the effects on the physics results.

Radiation protection and monitoring systems have been installed in several places throughout the detector. Silicon photodiodes are used to measure the radiation dosage. The SVT is the most susceptible to radiation damage. A set of six diodes are placed at both the forward and backward ends of the SVT. If instantaneous dose rates in the SVT diodes exceed a set threshold, the radiation protection system can limit the rate of beam injection or immediately abort both beams. Radiation monitors in the DCH will drop 


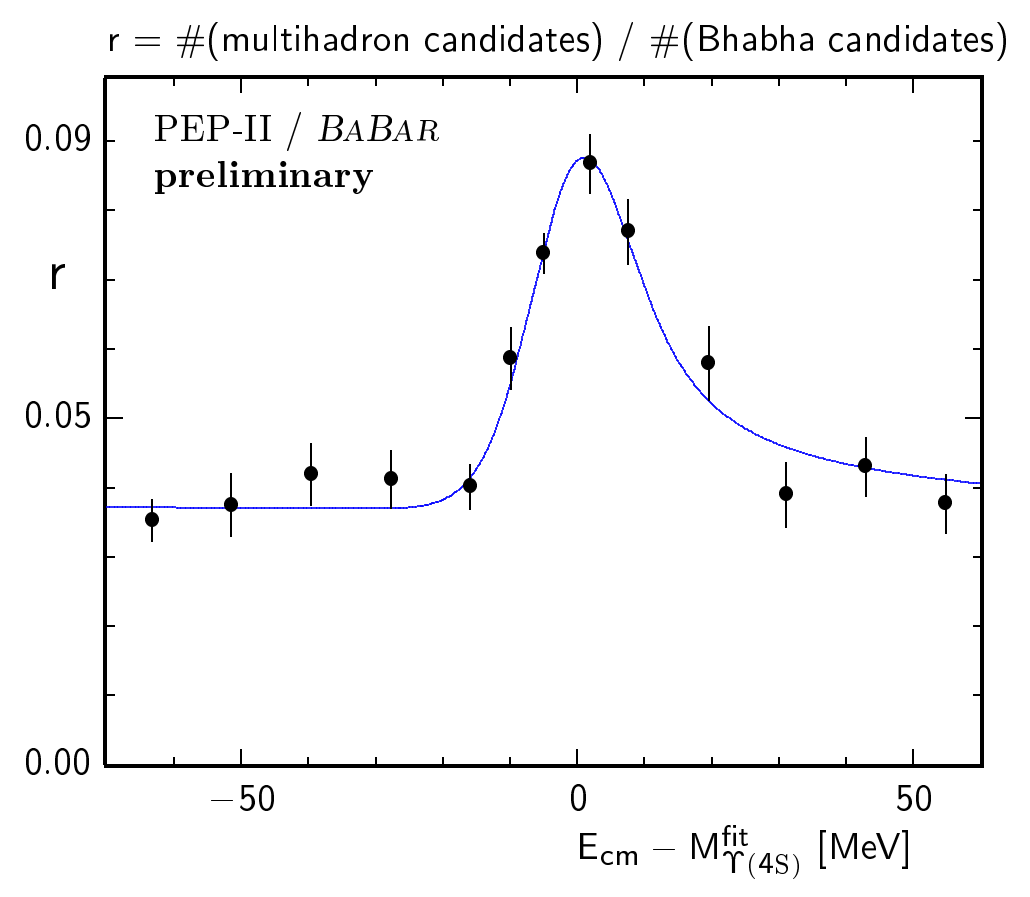

Figure 3.5: $\Upsilon(4 S)$ energy scan showing the ratio of multi-hadron events to Bhabha events.

the high voltage to a safe level under excessive dose rate. Further monitors are located on the EMC.

Filling of the beams from zero current takes about 10-15 minutes. The design currents for the HER and LER are $0.75 \mathrm{~A} \& 2.15 \mathrm{~A}$ respectively. PEP-II generally operates for about 40-50 minutes before needing to be topped-up to full current. This top-up procedure generally takes about three minutes.

The energy of the PEP-II beams is tunable. Generally the data are collected at the $\Upsilon(4 S)$ resonance. However, about $12 \%$ of the data are taken at $40 \mathrm{MeV}$ below the $\Upsilon(4 S)$ peak in order to study non- $B \bar{B}$ events referred to as continuum events. A scan of the resonance is shown in Figure 3.5. The peak energy was found by calculating the ratio of the detected multi-hadron events to the di-muon events. $40 \mathrm{MeV}$ below the $\Upsilon(4 S)$ peak is less than the energy threshold for $B \bar{B}$ production, so only lighter quarks and leptons are produced in these off-peak runs.

The relative luminosity is continuously monitored by measuring the rate of radiative Bhabha scattering $\left(e^{+} e^{-} \rightarrow e^{+} e^{-} \gamma\right)$. Absolute luminosity is calculated offline from other QED processes, primarily $e^{+} e^{-}$, and $\mu^{+} \mu^{-}$pairs (i.e. $e^{+} e^{-} \rightarrow \mu^{+} \mu^{-}$). For a data sample of $1 \mathrm{fb}^{-1}$, the statistical error on the measured luminosity is less than $1 \%$, and the systematic error is estimated to be around $1.5 \%$. This error is primarily due to the Monte Carlo generator and simulation of the detector.

The total number of $\Upsilon(4 S)$ events recorded by BABAR is estimated from multi-hadron and $\mu^{+} \mu^{-}$events in both on- and off-resonance data ${ }^{[32,33]}$ using the equation

$$
N_{\Upsilon(4 S)}=\left(N_{M H}-\kappa N_{\mu^{+} \mu^{-}} R_{o f f}\right) / \epsilon_{B \bar{B}},
$$


where $N_{M H}$ and $N_{\mu^{+} \mu^{-}}$are the numbers of multihadron and $\mu^{+} \mu^{-}$events respectively, $R_{\text {off } f}$ is the ratio of multi-hadron to $\mu^{+} \mu^{-}$events in off-peak data, $\kappa$ is a correction factor of $\mathcal{O}$ (1), and $\epsilon_{B \bar{B}}$ is the efficiency of $B \bar{B}$ events to satisfy the selection criteria. The $\Upsilon(4 S)$ is assumed to decay to $B \bar{B}$ pairs $100 \%$ of the time. The total number of $\Upsilon(4 S)$ mesons collected during the years $1999-2000$ is $(22.32 \pm 0.25) \times 10^{6}$ and forms the data sample used in this analysis.

\subsection{Silicon Vertex Tracker}

The innermost detector is the SVT. Its main purpose is to provide highly accurate charged particle detection, tracking and vertexing. It is critical for separation of the decay vertices of the $B$ and $D$ mesons and proper reconstruction of short lived particles. It is also needed for the measurement of low momentum particles such as the slow pion from the $D^{*}$ decay.

The SVT is made up of 5 layers of double-sided silicon microstrip detectors. The layers are organized into $6,6,6,16, \& 18$ modules respectively. Schematic views of the SVT are shown in Figures $3.6 \&$ 3.7. The strips on opposite sides of each layer are oriented orthogonally. One side has strips oriented parallel to the beam axis for $\phi$ measurement ( $\phi$ strips), and the other side has strips transverse to the beam axis $(z$ strips). The three inner layers primarily provide impact parameter measurements, while the two outer layers are needed for low $p_{t}$ tracking and extrapolating tracks to the $\mathrm{DCH}$.

The inner layers are planar, while the outer layers are arch shaped, as shown in

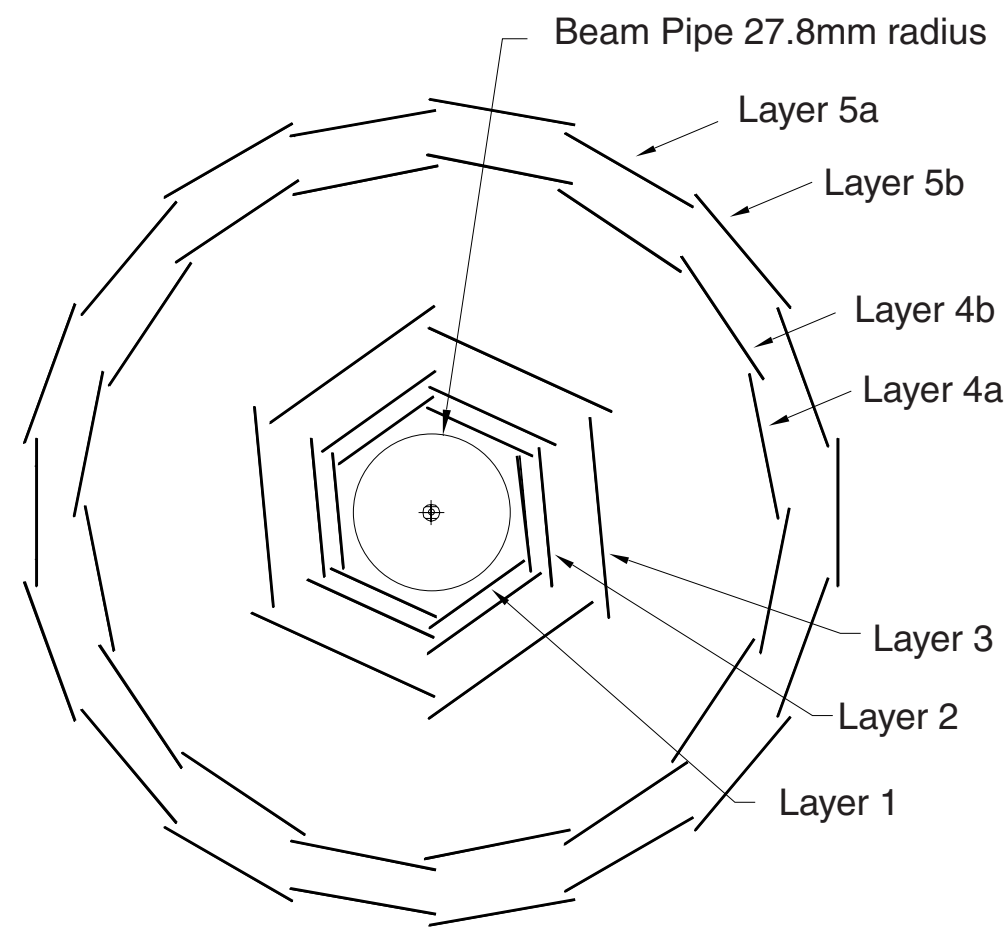

Figure 3.6: Schematic end view of the SVT. 


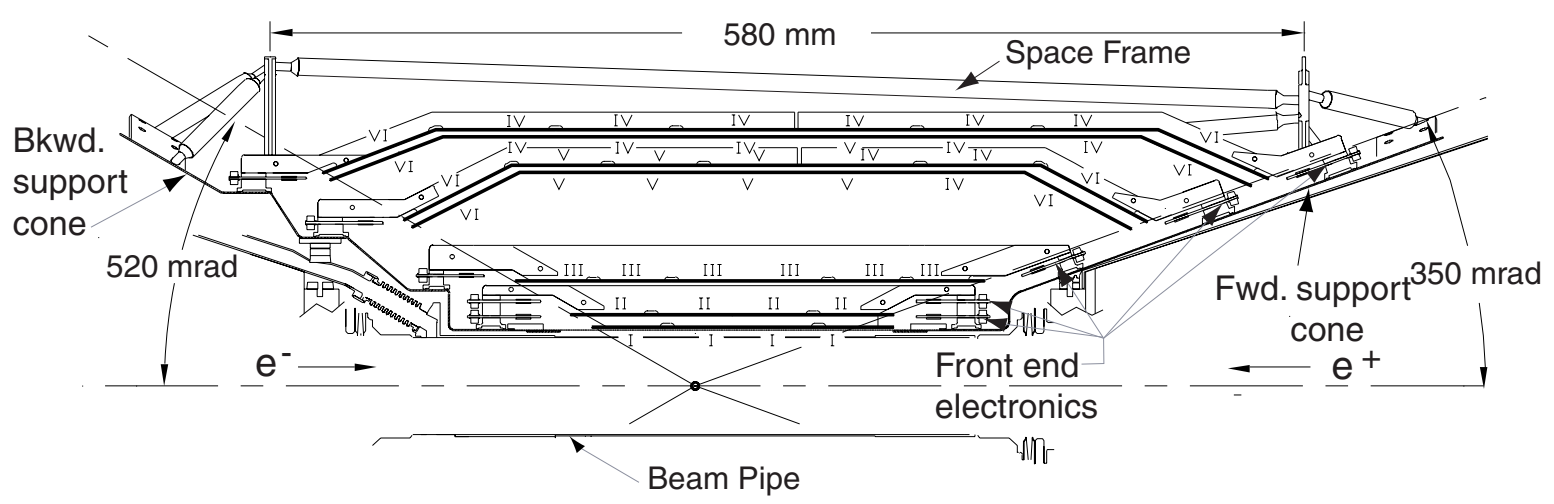

Figure 3.7: Side view of the SVT. The roman numerals represent the six different types of sensors.

Figure 3.7. This design was chosen to minimize the amount of material required to cover the solid angle, as well as increase the crossing angle for particles near the edge of the acceptance. Each layer has a different sensor shape in order to satisfy the geometric requirements of the detector. The smallest detectors are $43 \times 42 \mathrm{~mm}^{2}(\mathrm{z} \times \phi)$, and the largest are $68 \times 53 \mathrm{~mm}^{2}$. Identical trapezoidal sensors are added at each end of the outer two layers to form the arch shaped modules.

The modules are divided electrically into two half-modules which are read out at the ends. The $\phi$ strip sensors in each half-module are electrically connected with wire bonds to form a single readout strip. The total strip length is up to $140 \mathrm{~mm}(240 \mathrm{~mm})$ in the inner (outer) layers. Each $z$ strip for the inner 3 layers is connected to a separate preamplifier channel. The number of $z$ strips in layers 4 and 5 exceeds the number of available channels; therefore, two $z$ strips on different sensors are electrically paired to a single electronics channel. The length of each $z$ strip is about $50 \mathrm{~mm}$ for individual strips or $100 \mathrm{~mm}$ for paired strips. The pairing of two strips results in an ambiguity on the $z$ measurement, which is corrected by a pattern recognition algorithm. The strip pitch varies between $50 \mu \mathrm{m}$ and $210 \mu \mathrm{m}$ depending on the module and type $(z$ or $\phi)$ of strip. The total number of readout channels is approximately 150,000 .

The geometrical acceptance of the SVT is $90 \%$ of the solid angle in the CM system, and $86 \%$ is typically used in charged particle tracking. The total active silicon area is $0.96 \mathrm{~m}^{2}$, and the material traversed by particles is only $4 \%$ of a radiation length $\left(X_{0}\right)$. The combined hardware and software efficiency of the SVT is $97 \%$, excluding defective readout sections (9 of 208) which were damaged during installation. The efficiency is measured for each half-module by comparing the number of associated hits to the number of tracks crossing the active area of the module. With 5 layers of measurement, the defective modules have minimal impact on the physics analysis. 


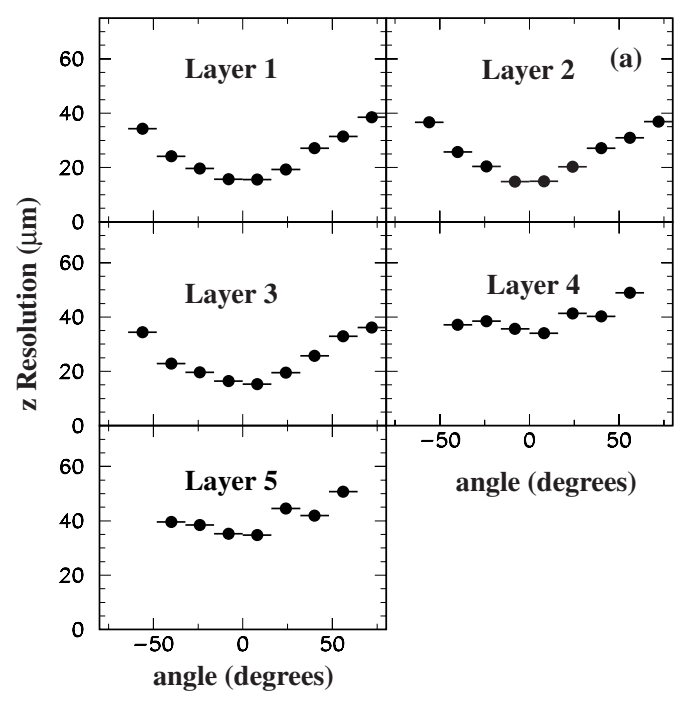

(a) $z$ resolution

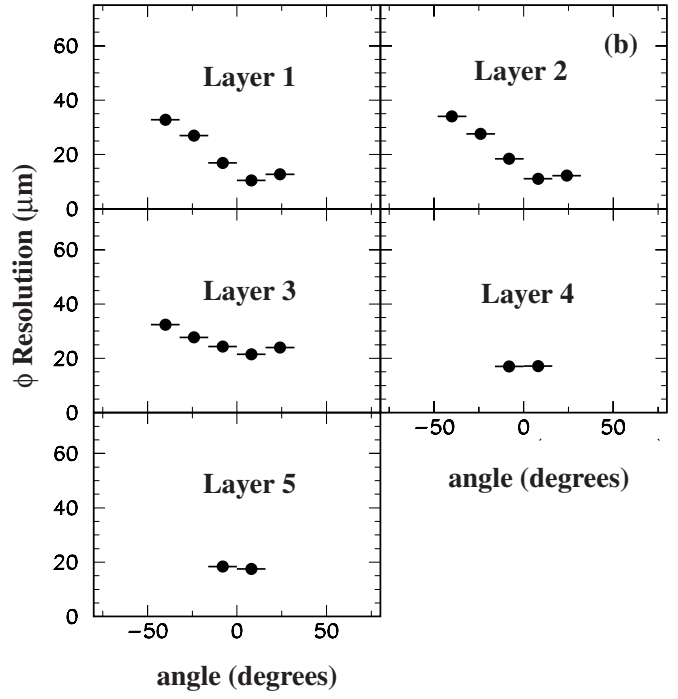

(b) $\phi$ resolution

Figure 3.8: SVT hit resolution in the a) $z$ and b) $\phi$ coordinate in microns as a function of track incident angle. There are fewer points in the $\phi$ resolution plots for the outer two layers because the range of incident angles subtended is much smaller.

The resolution of the SVT is shown is Figure 3.8. The plots of $\phi$ resolution in the first three layers show an asymmetry around $\phi=0$ because of the pinwheel design of the modules. As can be seen in the plots, the resolution for tracks at normal incidence is approximately $15 \mu \mathrm{m}(35 \mu \mathrm{m})$ for $z$ and $10 \mu \mathrm{m}(20 \mu \mathrm{m})$ for $\phi$ in the inner (outer) layers. With the boost of $\beta \gamma=0.56$, the $15 \mu \mathrm{m} z$ resolution produces a time resolution between decay vertices of 0.09 ps.

\subsection{Drift Chamber}

Surrounding the SVT is the drift chamber, which is the central tracking chamber for $B A B A R$. It provides efficient and high precision tracking of charged particles. These allow the reconstruction of $B, D$, and other mesons with little background. Some particles, such as the $K_{S}^{0}$, may decay inside the DCH, so there is no vertex information from the SVT. Therefore, the DCH needs to be able to measure not only the transverse momenta and positions, but also the longitudinal position of tracks to a resolution of $\sim 1 \mathrm{~mm}$. The DCH provides particle identification information for lower momentum particles by measuring the ionization loss $(\mathrm{d} E / \mathrm{d} x)$. It also supplies information for the charged particle trigger with a maximum time jitter of $0.5 \mu \mathrm{s}$. 


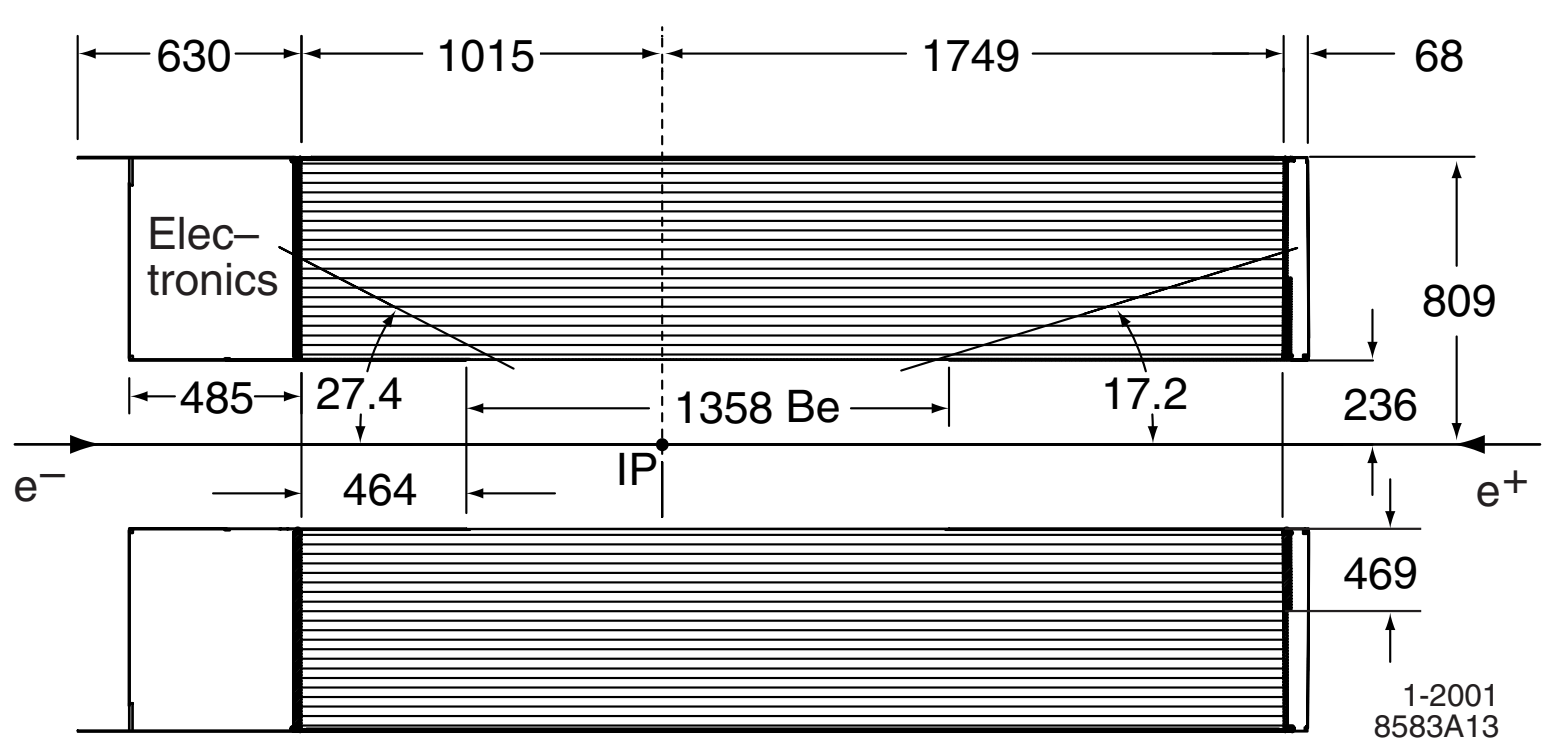

Figure 3.9: Schematic view of DCH with principle dimensions.

The main dimensions of the DCH are shown in Figure 3.9. The DCH consists of 40 layers of hexagonal drift cells grouped into 10 superlayers each consisting of four drift cells. The total number of cells in the detector is 7,104. This provides approximately 40 separate spatial and $\mathrm{d} E / \mathrm{d} x$ measurements for each charged particle passing through the DCH. Certain superlayers are oriented at small angles with respect to the $z$ axis to provide longitudinal position information. The orientation of the wires alternates between axial (A) superlayers and stereo (U,V) superlayers with positive and negative angles with respect to the $z$ axis. The order of the superlayers is AUVAUVAUVA. A schematic of the first few layers is shown in Figure 3.10.

There are four types of wires: sense, field, guard, and clearing. The specifications for each type of wire are shown in Table 3.2. Each cell consists of one sense wire surrounded by six field wires. The sense wires operate at a positive potential of $1960 \mathrm{~V}$, while the field wires are grounded. At the superlayer boundaries, the outermost field wire in each cell is replaced by two guard wires. These improve the electrostatic performance of the cell and match the gain of the boundary cells to those inside the superlayers. At the innermost boundary of layer one, and the outermost boundary of layer 40, two clearing

\begin{tabular}{|c|c|c|c|}
\hline Type & Material & $\begin{array}{c}\text { Diameter } \\
(\mu \mathrm{m})\end{array}$ & $\begin{array}{c}\text { Voltage } \\
(\mathrm{V})\end{array}$ \\
\hline Sense & $\mathrm{W}-\mathrm{Re}$ & 20 & 1960 \\
Field & $\mathrm{Al}$ & 120 & 0 \\
Guard & $\mathrm{Al}$ & 80 & 340 \\
Clearing & $\mathrm{Al}$ & 120 & 825 \\
\hline
\end{tabular}

Table 3.2: DCH wire specifications. 


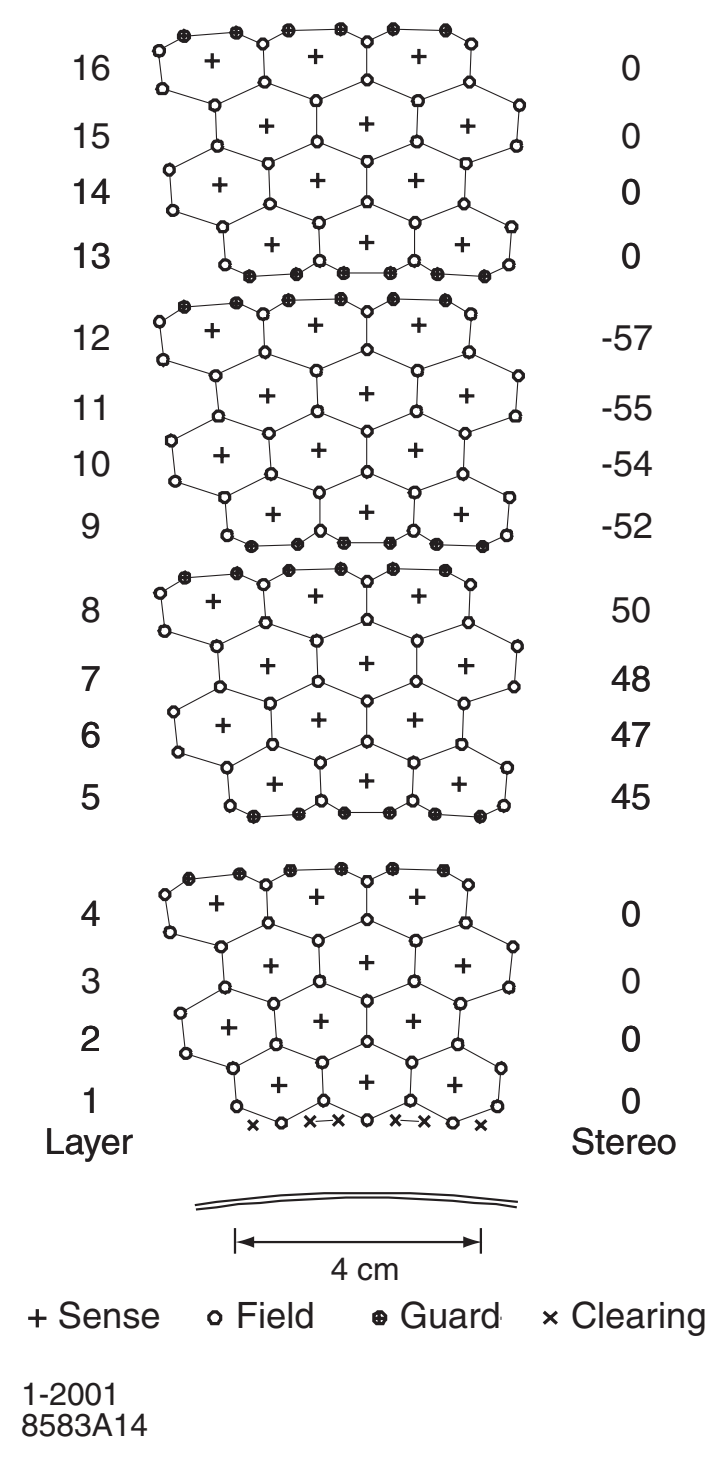

Figure 3.10: Schematic layout of drift cells for the first four superlayers. The numbers on the right side give the stereo angle in mrad of the sense wires in that layer. The lines around each drift cell are strictly an aid in visualization. The 1 mm thick beryllium inner wall is shown below the first layer.

wires are added per cell. These collect charges created through photon conversions within the walls. The sense wires are composed of tungsten-rhenium, $20 \mu \mathrm{m}$ in diameter. The other wires are made of aluminum. All wires are gold plated.

During commissioning, a small part of the DCH was damaged when $2 \mathrm{kV}$ was accidentally applied to the guard wires. This affected $10.4 \%$ of superlayer 5 and $4.2 \%$ of superlayer 6. During part of the 1999/2000 running, the DCH voltage was reduced to $1900 \mathrm{~V}$, out of concern for the damaged region. The wires in this region were disconnected when continuous discharge was observed over extended periods of time. The DCH showed a noticeable decrease in tracking efficiency at $1900 \mathrm{~V}$, so the voltage was later raised back to $1960 \mathrm{~V}$. 


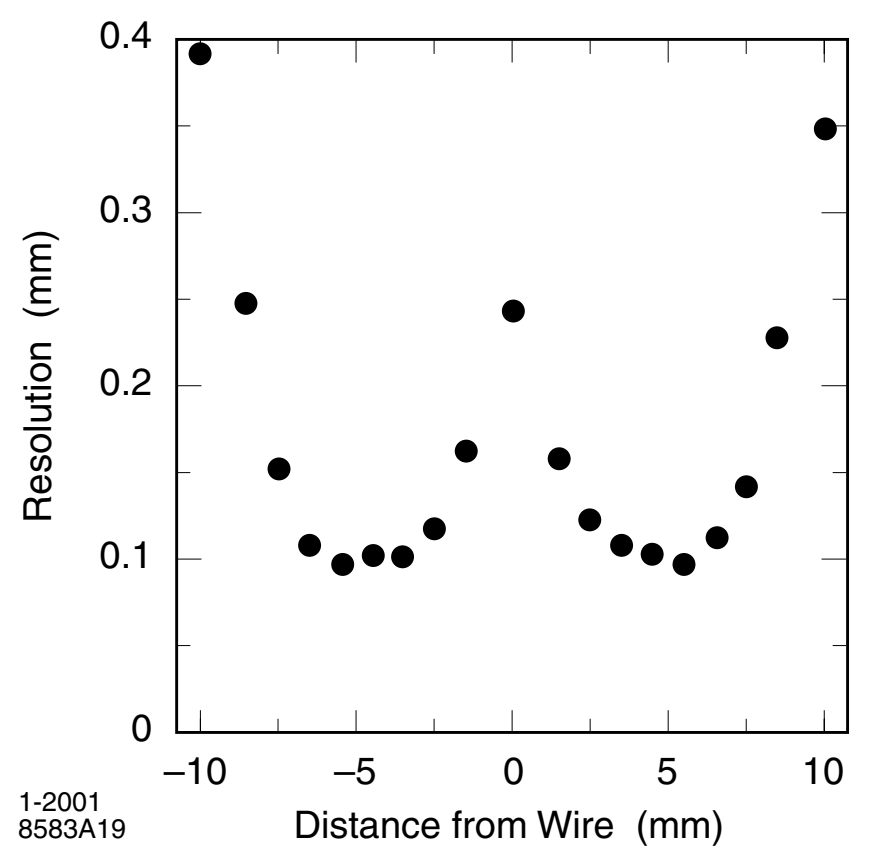

Figure 3.11: DCH position resolution vs. drift distance. Data are taken from layer 18 and averaged over all cells in the layer. Error bars are smaller than the points shown.

Particles require a transverse momentum of at least $120 \mathrm{MeV} / c$ to be reliably measured in the $\mathrm{DCH}$, and greater than $180 \mathrm{MeV} / c$ to pass through the drift chamber. With the forward length of the $\mathrm{DCH}$ of $1749 \mathrm{~mm}$, particles emitted with a polar angle of $17.2^{\circ}$ will cross at least half of the layers of the detector before passing through the front end plate. In backward direction, the length is $1015 \mathrm{~mm}$, resulting in a polar angle of $152.6^{\circ}$ required to cross half the layers.

Careful choice of materials was made to minimize multiple scattering. The gas used in the DCH is an 80:20 mixture of $\mathrm{He}: \mathrm{C}_{4} \mathrm{H}_{10}$, which has a radiation length five times larger than typical argon based gas mixtures. The inner wall is $1 \mathrm{~mm}$ thick beryllium. The total thickness of the DCH is only $1.08 \% X_{0}$ with $0.28 \% X_{0}$ from the inner wall and $0.2 \% X_{0}$ from the gas and wires.

The position resolution of the DCH for both the left and right side of the sense wire is shown in Figure 3.11. These measurements were derived using multi-hadron events and averaged over all cells in layer 18.

Figure 3.12 shows the $\mathrm{d} E / \mathrm{d} x$ measurements as a function of momenta for different particles. The $\mathrm{d} E / \mathrm{d} x$ resolution is typically $7.5 \%$. This provides at least $1 \sigma$ separation between $\pi$ and $K$ for momenta up to $700 \mathrm{MeV} / c$. 


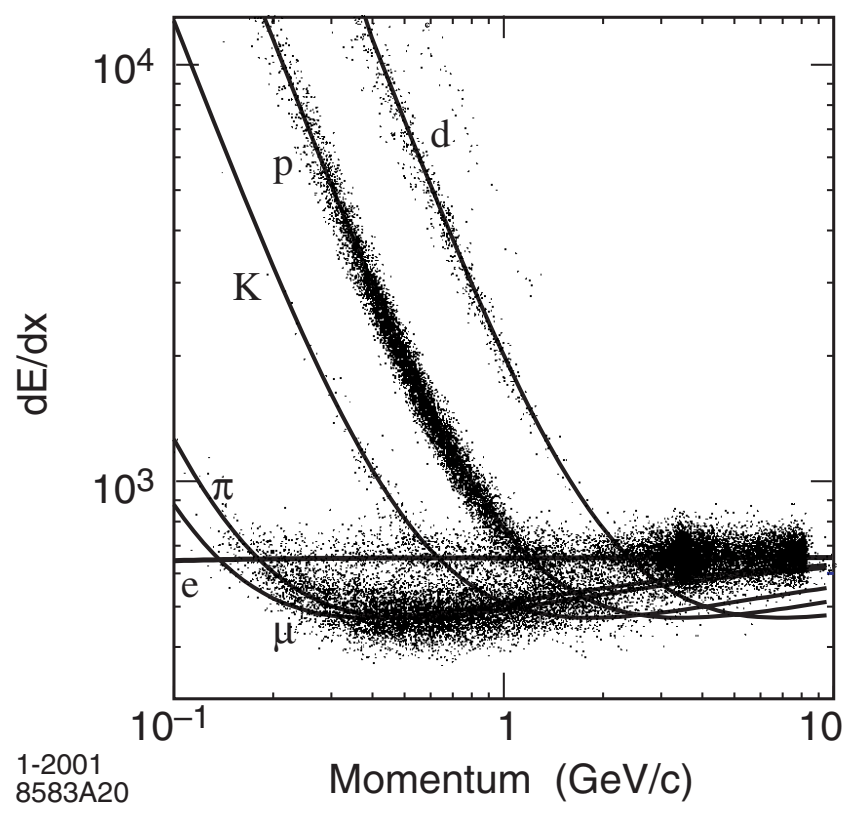

Figure 3.12: Measurements of $\mathrm{d} E / \mathrm{d} x$ vs. track momenta. The data include large samples of beam background triggers, as can be seen from the high rate of protons. The superimposed curves represent Bethe-Bloch predictions for different particle species.

\subsection{Cherenkov Detector}

Outside of the DCH is the detector of internally reflected Cherenkov light (DIRC). It is a ring-imaging Cherenkov detector with a novel approach to collecting the Cherenkov light. The main purpose of the DIRC is in kaon identification. It provides good $\pi / K$ separation from $0.7 \mathrm{GeV}$ to $4.2 \mathrm{GeV}$.

Cherenkov photons are emitted by a particle traveling faster than the speed of light in the ambient medium. The Cherenkov angle $\left(\theta_{C}\right)$ is related to the index of refraction $n$ and the speed of the charged particle $\beta=v / c$ through the relation

$$
\cos \theta_{C}=1 / n \beta \text {. }
$$

Therefore, by measuring the angle of the Cherenkov radiation, and the momentum of the track, the mass of the charged particle can be determined.

The DIRC relies upon total internal reflection (TIR) in order to read out the Cherenkov photons. Because of the requirement of little material and minimal radius before the EMC, the photomultiplier tubes (PMTs) must be placed out of the way at the back end of the detector. The DIRC is composed of long, thin quartz bars which serve as both the radiator material and the light guides for the Cherenkov photons. Figure 3.13 shows a representation of light production, transport and imaging. Through TIR, the photons produced will be reflected out to the end of the detector where the PMTs are located. Since angles are maintained upon reflection, the Cherenkov angle is preserved. 
The bars are made from a synthetic fused silica and have a rectangular cross section. The index of refraction of the bars is 1.473. The DIRC bars are arranged in a 12-sided polygonal barrel. Each side consists of 12 bars, each $33 \mathrm{~mm}$ wide, for a total of 144 bars. Each bar on a side is optically isolated by a $150 \mu \mathrm{m}$ air gap. For particles with $\beta \simeq 1$, some Cherenkov photons will always lie within the TIR limit, even for particles at normal incidence. The photons will be transported to either end of the bar, and a mirror is placed at the forward end to reflect the photons to the instrumented rear.

The imaging region consists of a water filled expansion region, called the standoff box. It is made of stainless steel, and consists of 12 sectors instrumented with PMTs. It contains 6,000 litres of purified water, with an average index of refraction, $n \simeq 1.346$, which is reasonably close to that of the fused silica. This minimizes the reflection at the silica-water interface. Each PMT sector contains 896 PMTs of $29 \mathrm{~mm}$ diameter in a closely packed array inside the water volume. A hexagonal light-catcher cone is mounted in front of each PMT to increase the active surface area light collection fraction to about $90 \%$.

The silica bars subtend a solid angle corresponding to $94 \%$ of the azimuth and $83 \%$ of the CM polar angle. The geometric contribution to the single photon Cherenkov angle

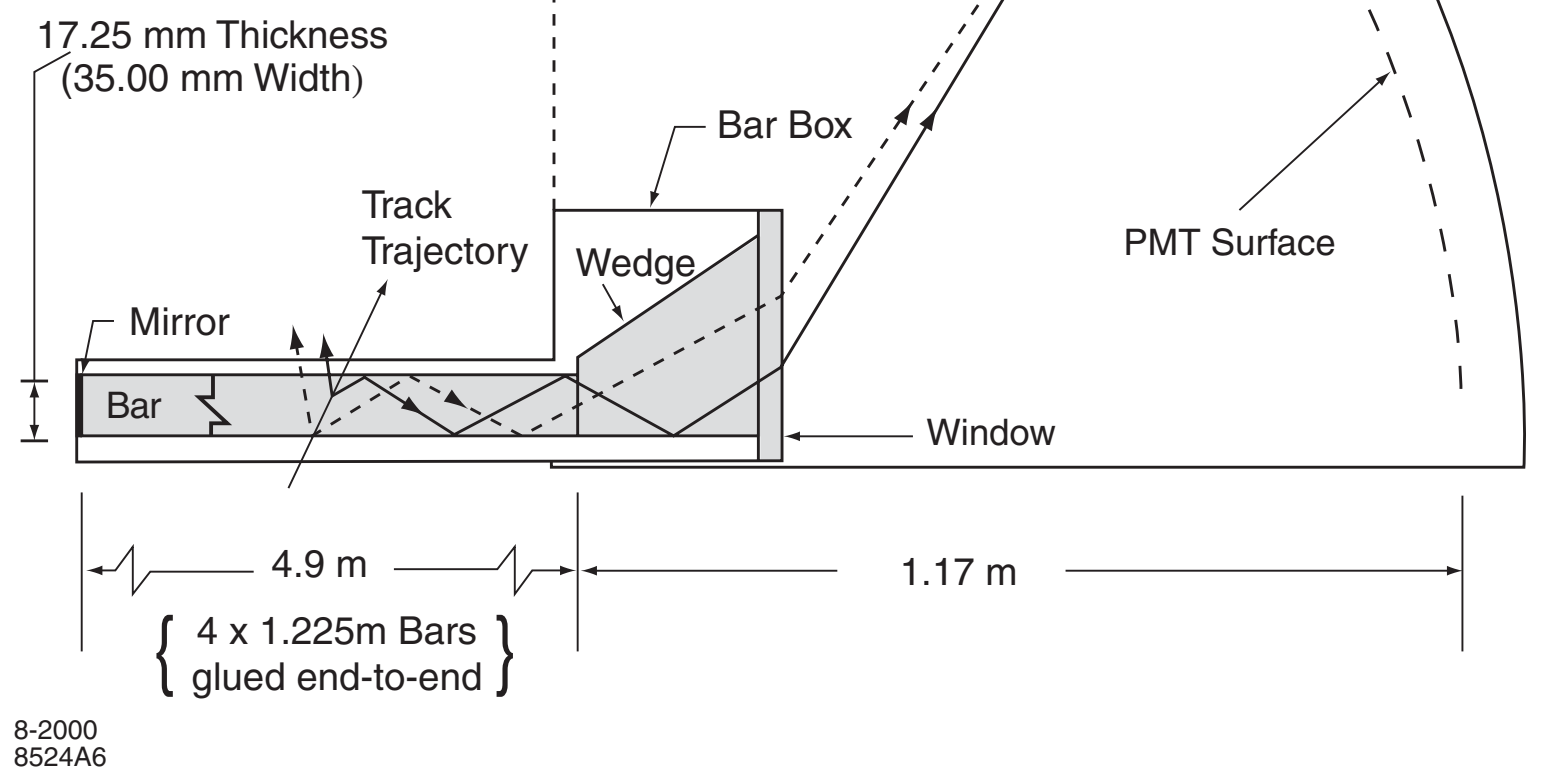

Figure 3.13: Schematics of the DIRC fused silica bar and imaging region. 


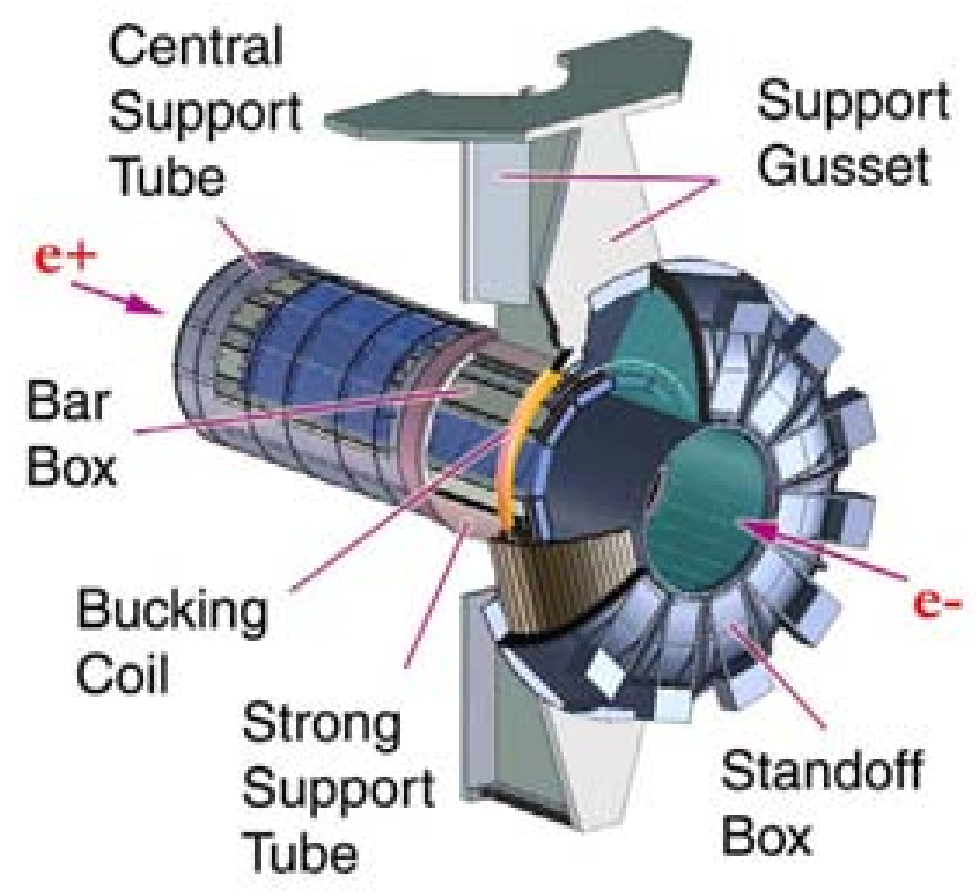

Figure 3.14: View of DIRC system.

resolution is $\sim 7 \mathrm{mrad}$, which originates from the size of the bars and the PMTs. This value is slightly larger than the rms spread of the photon production and transmission dispersions of $\sim 5.4 \mathrm{mrad}$. The overall single photon resolution is estimated to be about 10 mrad.

The photons can make up to several hundred bounces before reaching the standoff box. The bar surfaces are designed to have an internal reflection coefficient greater than 0.9992 per reflection, so about $80 \%$ of the light is maintained. The dominant contribution to the overall detection efficiency comes from the quantum efficiency of the PMTs: $\mathcal{O}(20 \%)$. For a typical $\beta=1$ particle entering the centre of a bar at normal incidence, the expected number of photoelectrons is $\sim 28$. This increases by over a factor of two in the forward and backward direction.

In order to identify the type of particle $(e, \mu, \pi, K, p)$, an unbinned maximum likelihood method is used to incorporate all the space and time information from the DIRC. A likelihood value is calculated for each of the five long-lived particles for a track crossing through the active area of the DIRC. The expected separation between kaons and pions is about $4.2 \sigma$ at $3 \mathrm{GeV} / c$ and about $2.5 \sigma$ at $4.2 \mathrm{GeV} / c$.

\subsection{Electromagnetic Calorimeter}

Energy measurements are made by the electromagnetic calorimeter (EMC). It is designed to measure electromagnetic showers over the range of $20 \mathrm{MeV}$ to $9 \mathrm{GeV}$ with high 


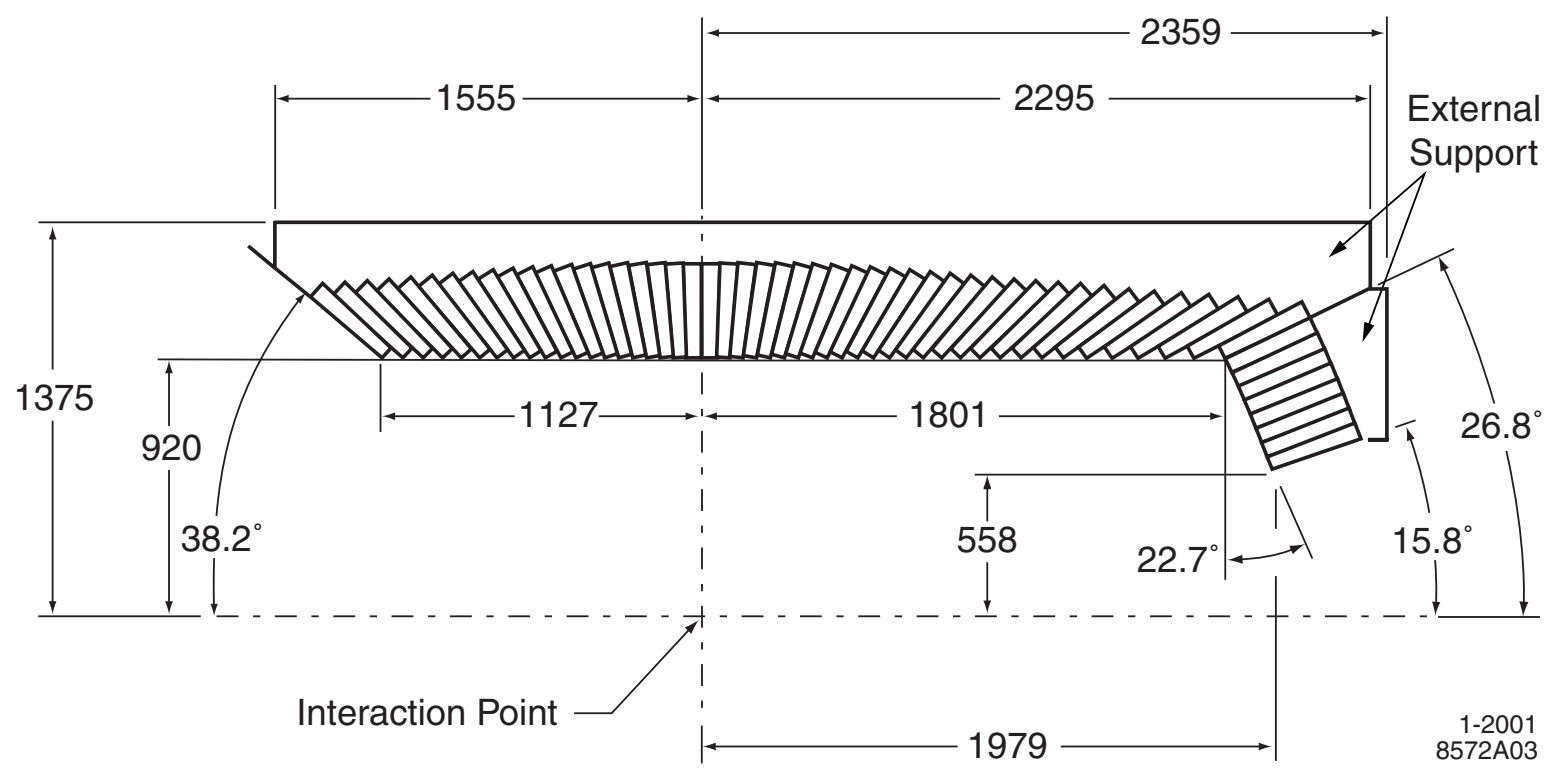

Figure 3.15: Cross sectional view of the top half of the EMC showing the arrangement of the 56 rings of CsI crystals. The detector is axially symmetric around the $z$-axis. All dimensions are given in $\mathrm{mm}$.

efficiency, and excellent energy and angular resolution. This is necessary to allow the detection of photons from $\pi^{0}$ and $\eta$ decays as well as from electromagnetic and radiative processes. The EMC is also important in the PID system for electrons and muons.

The EMC is composed of 6,580 thallium-doped cesium iodide crystals. The crystals absorb electromagnetic showers and produce scintillation light which is read out with silicon photodiodes matched to the spectrum of the light. The EMC is divided into two sections: the barrel, which consists of 5760 crystals in 48 rings, and the endcap, which contains 820 crystals in eight rings. The arrangement of the rings is shown in Figure 3.15. The EMC has full azimuthal coverage, and extends in polar angle from $15.8^{\circ}$ to $141.8^{\circ}$. This corresponds to a solid-angle coverage of $90 \%$ in the CM system.

The crystals are made from CsI salt doped with $0.1 \%$ Tl. They are machined into tapered trapezoids. The transverse dimensions of the trapezoids vary for each of the 56 rings in order to provide hermetic coverage. The crystals within any ring are identical. The typical area of the front face is $4.7 \times 4.7 \mathrm{~cm}^{2}$, and the back face area is typically $6.1 \times 6.0 \mathrm{~cm}^{2}$. The length of the crystals increases from $29.6 \mathrm{~cm}$ in the backward direction to $32.4 \mathrm{~cm}$ in the forward direction. A typical crystal is shown in Figure 3.16 .

Each crystal is wrapped in two layers of diffuse white reflector, each $165 \mu \mathrm{m}$ thick, which helps to recover any light transmitted through the polished surface of the crystal. It is further wrapped in $25 \mu \mathrm{m}$ thick $\mathrm{Al}$ foil which is electrically connected to the metal housing of the photodiode/preamplifier assembly to provide a Faraday shield. A layer of $13 \mu \mathrm{m}$ thick mylar is added to provide electrical insulation from the external support. The crystal assemblies are inserted into carbon-fibre-epoxy composite (CFC) modules which are supported individually from an external support structure. 


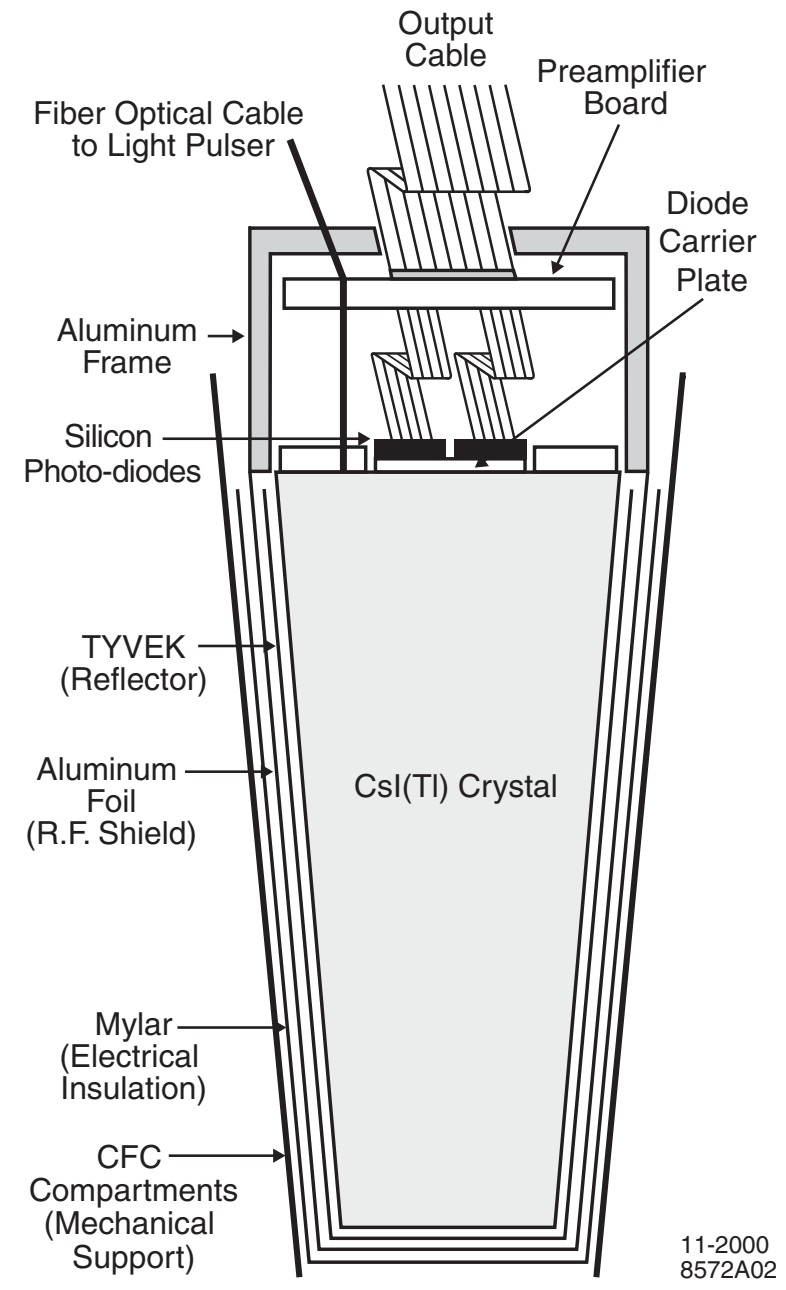

Figure 3.16: Diagram of a $\mathrm{CsI}(\mathrm{Tl})$ crystal with wrapping and readout electronics. This drawing is not to scale.

The photons from electromagnetic showers within the crystals are detected by two $2 \times 1 \mathrm{~cm}^{2}$ silicon PIN diodes. The light yield is required to be uniform to within $\pm 2 \%$ in the front half of the crystal. This limit increases linearly to a maximum of $\pm 5 \%$ at the rear face. The photodiodes have a quantum efficiency of $85 \%$ for the $\mathrm{CsI}(\mathrm{Tl})$ scintillation light. During the quality control process, the light yield of every crystal/photodiode assembly was measured using a ${ }^{88} \mathrm{Y}$ radioactive source. The resulting signal distribution had a mean and rms width 7300 and 890 photoelectrons/MeV, respectively. None of the crystals had a signal of less than 4600 photoelectrons/MeV.

An electromagnetic shower typically spreads over several crystals, forming a cluster of energy deposits. The definition of a cluster requires that at least one crystal contain an energy above $10 \mathrm{MeV}$. Contiguous neighbours (including corners) of a crystal with at least $3 \mathrm{MeV}$ are considered part of the cluster, as are surrounding crystals with energy above $1 \mathrm{MeV}$. Pattern recognition algorithms have been developed to efficiently recognize clusters and differentiate merged clusters with more than one energy maximum, called 
bumps. To determine whether a bump is associated with a charged particle, the track is projected to the inner face of the EMC. The distance between the bump centroid and the track impact point is calculated, and if it is consistent with the angle and momentum of the track, the bump is associated with the charged particle. Otherwise, the bump is assumed to come from a neutral particle.

Several different sources are used to determine the energy resolution of the EMC. At low energies, it is measured directly using radioactive sources. Below $2 \mathrm{GeV}$ it is measured from the mass resolutions of $\pi^{0}$ and $\eta$ mesons decaying into two photons of equal energy in their rest frame. It can also be measured at about $500 \mathrm{MeV}$ from the decay of $\chi_{c 1} \rightarrow J / \psi \gamma$. At high energies, Bhabha scattering is used. The energy dependence of the energy resolution can be parametrized by

$$
\frac{\sigma_{E}}{E}=\frac{(2.32 \pm 0.30) \%}{\sqrt[4]{E(\mathrm{GeV})}} \oplus(1.85 \pm 0.12) \% .
$$

The $\pi^{0}$ mass resolution reconstructed from two photons over the full energy range of $B \bar{B}$ events is $6.9 \mathrm{MeV} / c^{2}$.

The measurement of angular resolution uses the analysis of $\pi^{0}$ and $\eta$ decays to two photons of approximately equal energy. The resolution varies between about $12 \mathrm{mrad}$ at low energies and $3 \mathrm{mrad}$ at high energies. The energy dependence of the angular resolution can be parametrized by

$$
\sigma_{\theta}=\sigma_{\phi}=\frac{3.87 \pm 0.07}{\sqrt{E(\mathrm{GeV})}}+(0.00 \pm 0.04) \mathrm{mrad} .
$$

The shower energy and lateral shower moments provide information for separating electrons from charged hadrons. The most important variable in the discrimination of hadrons is the ratio of the shower energy to the track momentum $(E / p)$. Electrons generally deposit all of their energy in the EMC, giving a $E / p$ ratio a peaked at 1 . Hadrons tend to have a lower ratio due to nuclear interactions. Muons deposit only a minimum of energy in the EMC; this fact is used to veto out particles depositing larger energies. Also, the shower shape created by an electron is generally narrower than those produced by hadrons. A detailed description of the PID techniques and efficiencies is described in Section 4.2.

\subsection{Main Solenoid}

The BABAR magnet system consists of a superconducting solenoid, a segmented flux return, and a field compensating coil called the bucking coil. The magnetic field produced allows for charged particle momentum measurement. The segmented flux return serves as a hadron absorber for hadron/muon separation as well as providing the overall structure and support for the detector components. The flux return will be described in detail in Section 3.8. The key components of the magnet system can be seen in Figures $3.1 \& 3.2$.

\footnotetext{
${ }^{\text {a }}$ Technically, this should be $E / p c$, but by convention, $c \equiv 1$, so it is dropped.
} 
The central field is produced by a $1.5 \mathrm{~T}$ solenoid. The superconducting material is composed of niobium-titanium ( $46.5 \% \mathrm{Nb}$ by weight) filaments, each less the $40 \mu \mathrm{m}$ in diameter. The filaments are wound into $0.8 \mathrm{~mm}$ strands, 16 of which are formed into Rutherford cable measuring $1.4 \times 6.4 \mathrm{~mm}$. The conducting coil is made of Rutherford cable co-extruded with pure aluminum stabilizer. The coil is maintained at a temperature of $4.5 \mathrm{~K}$ by a liquid helium cryostat located on top of the BABAR detector. The operating current is $4596 \mathrm{~A}$.

The bucking coil reduces the leakage fields into the PEP-II components and the DIRC photomultipliers. It is a conventional, water-cooled, 10 layer, copper coil consisting of 140 turns. The operating current is 200 A. However, a current of up to $575 \mathrm{~A}$ can be applied, if needed, in order to demagnetize the DIRC shield.

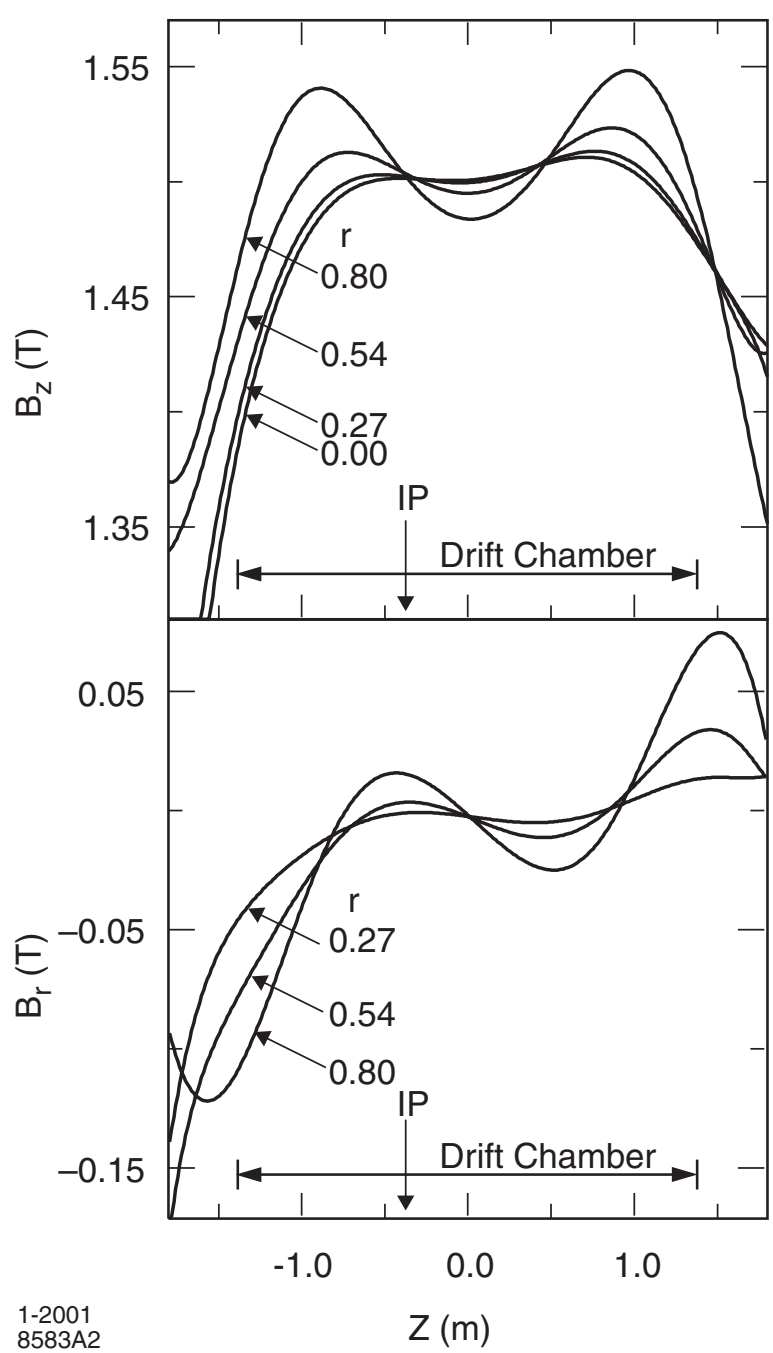

Figure 3.17: Magnetic field components $B_{z}$ and $B_{r}$ as a function of $z$. The curves represent measurements at different radial distances, $\mathrm{r}$ (metres). The extent of the DCH and the IP are indicated. 
The PEP-II bending and focusing magnets B1 \& Q1 are located inside the solenoid (see Figure 3.1). These samarium-cobalt permanent magnets cannot sustain a large radial magnetic field. Therefore, the radial component of the field at a radius $r=200 \mathrm{~mm}$ is kept below $0.25 \mathrm{~T}$. The Q2, Q4 \& Q5 magnets are exposed to stray fields which are less than $0.01 \mathrm{~T}$ averaged over their apertures.

The magnetic field is mapped by movable Hall probes. There are five sets of $B_{r}$ and $B_{z}$ probes, and two $B_{\phi}$ probes. Also, an NMR probe was mounted at a radius of $89 \mathrm{~mm}$ to provide a very precise field reference near the $z$-axis as a function of $z$. In the tracking volume, the field is very uniform. Figure 3.17 shows the field components $B_{z}$ and $B_{r}$ as a function of $z$ for various radial distances. The $B_{\phi}$ component does not exceed $1 \mathrm{mT}$. The variation of the bending field for a high momentum track is less than $2.5 \%$ within the tracking region.

\subsection{Instrumented Flux Return}

The instrumented flux return (IFR) for the main magnet also serves as the principle detector for identifying muons and neutral hadrons (primarily $K_{L}^{0}$ and neutrons). It was designed to have a high efficiency and good purity over a wide range of momenta down to less than $1 \mathrm{GeV} / c$ and a high solid angle coverage. The IFR consists of up to 19 alternating layers of steel and resistive plate chambers (RPCs). It uses the steel of the flux return as a muon filter and hadron absorber. Muons can be identified by measuring

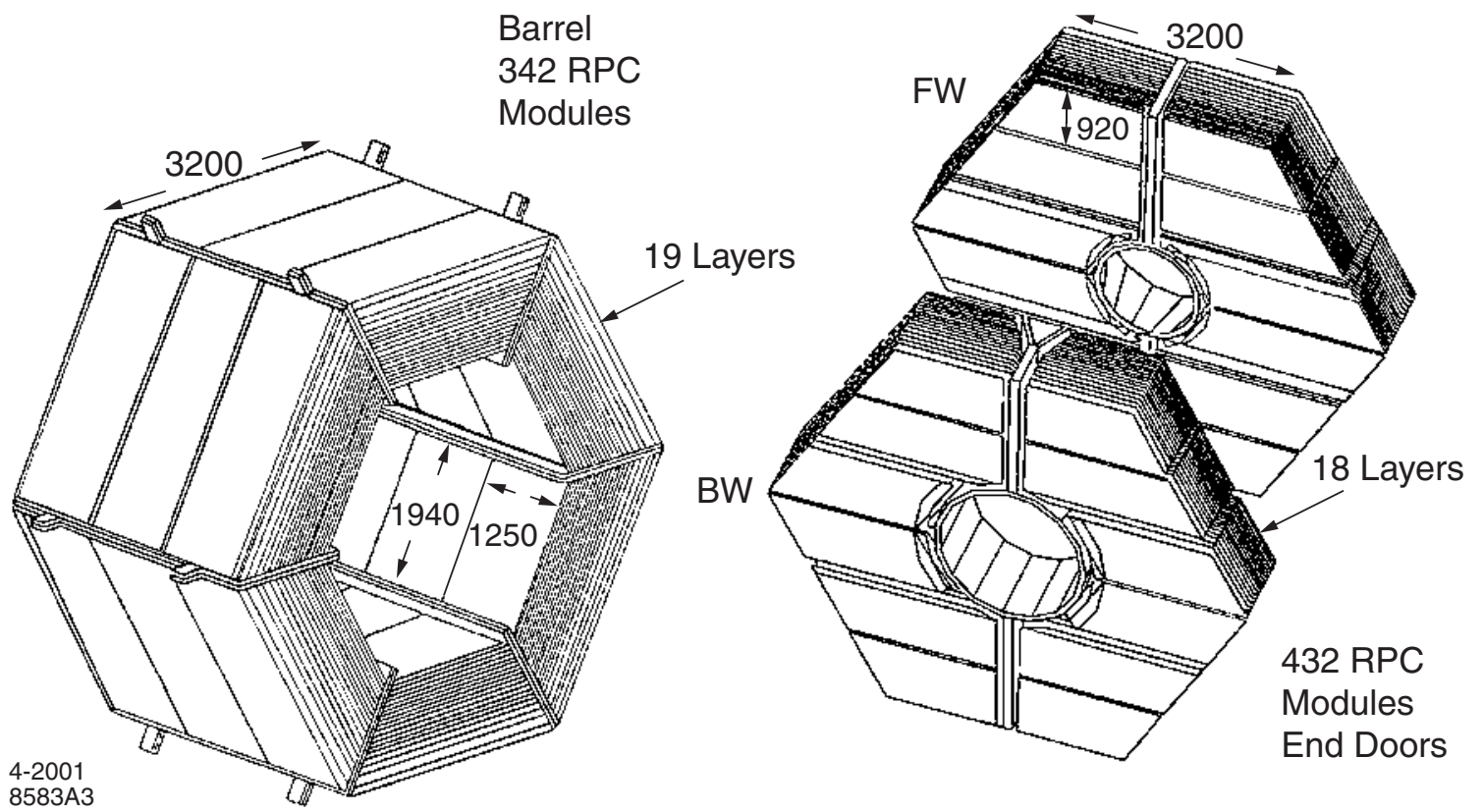

Figure 3.18: Schematic view of the IFR showing the barrel sectors and the forward(FW) and backward(BW) end doors. The shape and dimensions of the RPC modules are indicated. 


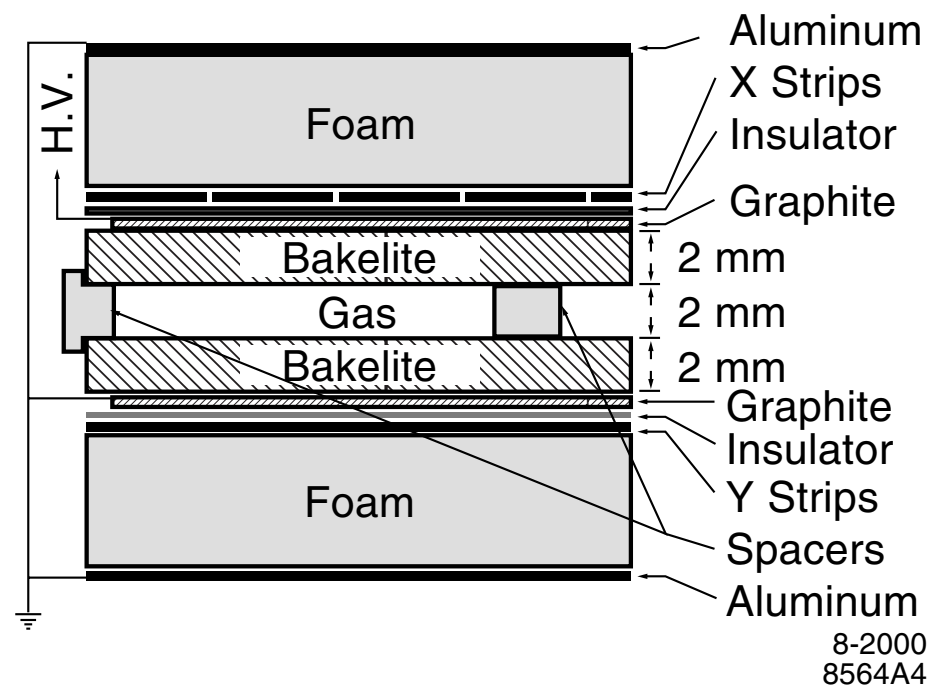

Figure 3.19: Cross section of a planar RPC with the schematics of the high voltage (HV) connection.

how many layers of steel the particle traverses, and comparing that to the expected radiation length for a muon.

The flux return is made of three components: the barrel section and doors on the forward and backward ends. The doors are split vertically and can be rolled aside to allow some access to the inner detectors. The main barrel component is hexagonal and segmented into 18 layers of steel. The thickness of the plates increases from $2 \mathrm{~cm}$ for the inner nine plates to $10 \mathrm{~cm}$ for the outermost plates. The gap between plates is $3.5 \mathrm{~cm}$ in the inner layers of the barrel and $3.2 \mathrm{~cm}$ elsewhere. Planar RPCs are inserted before and after each plate making a total of 19 RPC layers in the barrel. The endcaps have 18 RPC layers. Additionally, two layers of cylindrical RPCs are installed between the EMC and the magnet cryostat to detect particles exiting the EMC. There are a total of 806 RPC modules, 57 in each of the six barrel sectors, 108 in each of the four half-end doors, and 32 in the two cylindrical layers.

The planar RPCs are made from two Bakelite (phenolic polymer) sheets, $2 \mathrm{~mm}$ thick. These are separated by a $2 \mathrm{~mm}$ gap which is filled with a gas mixture of $56.7 \%$ argon, $38.8 \%$ Freon, and $4.5 \%$ isobutane. The inner surfaces of the Bakelite are coated with linseed oil, except on the cylindrical RPCs. The outer surfaces are coated with graphite and connected to high voltage $(\sim 8 \mathrm{kV})$ and ground, and protected by an insulating mylar film. The RPCs detect streamers from ionizing particles via capacitive readout strips. The width of the strips varies between 16.0 and $38.5 \mathrm{~mm}$ depending on the location of the RPC. A cross section of an RPC is shown in Figure 3.19.

The efficiency of the RPCs is evaluated for both $e^{+} e^{-}$collision data and cosmic ray muons. To calculate the efficiency in a particular chamber, nearby hits in a given layer and hits in different layers are combined to form clusters. Two-dimensional clusters are formed by joining one-dimensional clusters (of the same readout coordinate) in different layers. The residual distributions from straight line fits to two-dimensional clusters typically 
have an rms width of less than $1 \mathrm{~cm}$. An RPC strip is considered efficient if a signal is detected at a distance of less than $10 \mathrm{~cm}$ from the fitted particle trajectory in either of the two readout planes. Given that there is a problem in the RPC efficiency, as described in the next section, this method for calculating efficiency has become biased. A new method using muon pairs is being developed to measure the RPC efficiency.

\subsubsection{IFR Efficiency Degradation}

$B A B A R$ has been experiencing a gradual loss in IFR efficiency since the start of the experiment. Many of the RPCs have slowly been losing their ability to detect passing particles. This has appeared in the data as a loss in muon identification efficiency, as can be seen in Figure 3.20, and it was noticeable within the first year of running.

This problem has been considerably studied within BABAR. ${ }^{[35]}$ The direct cause of the problem is in the linseed oil coating the resistive plates. Some flow of the oil has caused connections (bridges) to form across the plates of the chamber. This has reduced the resistance between the plates by about a factor of 40 and greatly reduced their ability to produce a signal when a particle passes through the area surrounding the bridge. The

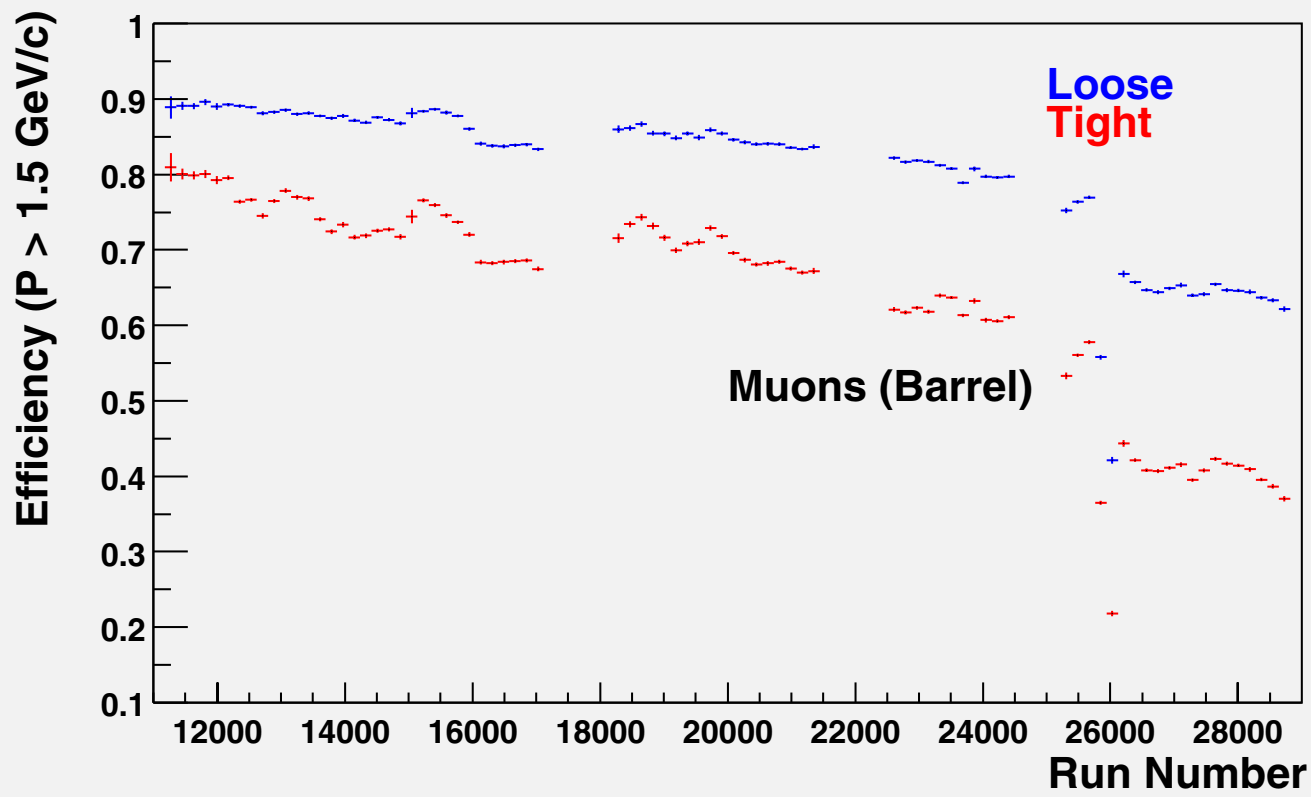

Figure 3.20: Muon detection efficiency vs. run number for data collected from 19992002. ${ }^{[34]}$ Runs in the the $1999 / 2000$ run period are numbered approximately 10,000 to 17,000. The labels Loose and Tight refer to the muon identification algorithms described in Section 4.2.1. The Tight mode (lower line) shows a worse decline, and is more important to this analysis. Around run number 26,000 there was a temporary failure in the IFR front-end electronics. 
production of these bridges has been showing up in certain RPCs more readily than in others, and it is suspected to be related to the amount of oil used to coat the RPC plates during production.

This problem hasn't shown up in previous experiments using similarly constructed RPCs, such as the L3 experiment at CERN. However, due to the California climate, and the closer proximity of the BABAR detector to ground level, the temperature of the detector tends to be significantly higher during the summer than at other experiments. This increased temperature reduced the linseed oil viscosity to the point where it could flow into the gap between the plates, and under the electrostatic forces, create the bridges between the plates.

Numerous methods have been studied to try to fix the problem with the detectors in situ, but none have been found. The solution proposed by BABAR is to replace the RPCs in the barrel region with limited streamer tubes, which are a different detector technology and not subject to this problem. For the existing data, the steady decline in muon efficiency can cause a systematic effect in the physics results unless treated during the data processing.

\subsection{Trigger}

The trigger system is designed to select events of interest from the large number of background events with a high, stable, and well understood efficiency. At design luminosity, the beam-induced background rates are typically about $20 \mathrm{kHz}$. The trigger keeps the total event rate under $120 \mathrm{~Hz}$. Types of events and their rates are shown in Table 3.3. The total trigger efficiency is required to exceed $99 \%$ for all $B \bar{B}$ events and at least $95 \%$ for continuum events.

The trigger is designed as a two level system. Level 1 (L1) is a hardware trigger, and Level 3 (L3) is software based. There is a possibility in the design to incorporate a Level 2 trigger at a future date, should it be needed.

\begin{tabular}{|c|r|r|r|}
\hline $\begin{array}{c}\text { Event } \\
\text { type }\end{array}$ & $\begin{array}{r}\text { Cross } \\
\text { section } \\
(\mathrm{nb})\end{array}$ & $\begin{array}{r}\text { Production } \\
\text { Rate (Hz) }\end{array}$ & $\begin{array}{r}\text { Level 1 } \\
\text { Trigger } \\
\text { Rate (Hz) }\end{array}$ \\
\hline$b \bar{b}$ & 1.1 & 3.2 & 3.2 \\
other $q \bar{q}$ & 3.4 & 10.2 & 10.1 \\
$e^{+} e^{-}$ & $\sim 53^{*}$ & 159 & 156 \\
$\mu^{+} \mu^{-}$ & 1.2 & 3.5 & 3.1 \\
$\tau^{+} \tau^{-}$ & 0.9 & 2.8 & 2.4 \\
\hline
\end{tabular}

Table 3.3: Cross sections, production and trigger rates for the principal physics processes at $10.58 \mathrm{GeV}$ for a luminosity of $3 \times 10^{33} \mathrm{~cm}^{-2} \mathrm{~s}^{-1}$. ${ }^{*}$ The $e^{+} e^{-}$cross section refers to events with either the $e^{+}, e^{-}$, or both inside the EMC detection volume. 
The L1 trigger is configured to have an output rate of $1 \mathrm{kHz}$ in normal operation. Triggers are produced within a fixed latency window of 11-12 $\mu$ s after the $e^{+} e^{-}$collision and delivered to the Fast Control and Timing System. The L1 decision is based on charged tracks in the DCH above a preset transverse momentum, showers in the EMC, and tracks detected in the IFR. The DCH trigger looks for track segments in groups of eight neighbouring cells. The EMC trigger groups sets of crystals (24 for the barrel, and 19-22 in the endcap) into towers. For each tower, crystal energies above $20 \mathrm{MeV}$ are summed. The IFR trigger looks for hits in the 10 IFR sectors $(6$ barrel sextants and 4 half-endcap doors). It is used for triggering on $\mu^{+} \mu^{-}$and cosmic ray events.

The L3 trigger receives the output from L1, performs a further rate reduction for the main physics sources, and flags the special categories of events needed for luminosity determination, diagnostic, and calibration purposes. The L3 software runs on a dedicated online computer farm. It has access to the entire event for making decisions, and operates by refining and augmenting the selection methods used in L1. For example, better DCH tracking and vertex resolution, and EMC clustering filters allow for greater rejection of beam backgrounds and Bhabha events. Of the L3 output, the acceptance for physics events is $\sim 90 \mathrm{~Hz}$, while $\sim 30 \mathrm{~Hz}$ is used for special event categories. For a typical run on the $\Upsilon(4 S)$ peak with an average luminosity of $2.6 \times 10^{33} \mathrm{~cm}^{-2} \mathrm{~s}^{-1}$, the desired physics events contribute $13 \%$ of the total output, while the calibration and diagnostic output samples comprise $40 \%$.

Output from L3 is sent to the Online Prompt Reconstruction (OPR) software, which does the primary tracking and PID analysis on the data. It is designed to process the data within a few hours of its acquisition and provide the data collections to be used by the BABAR community. OPR produces a number of boolean variables which roughly identify the type of decay and the associated particles in the event, and provides various lists and data structures which greatly enhance the speed and efficiency of general physics analyses. 


\section{Chapter 4}

\section{Charged Particle Tracking and Identification}

\subsection{Charged Particle Tracking}

A charged particle travels in a helical trajectory through a magnetic field. The methods for determining the helix parameters and their resolutions are discussed.

\subsubsection{Reconstruction}

The tracks are defined by five helix parameters:

- $z_{0}$ : the distance from the origin to the point of closest approach along the $z$-axis.

- $d_{0}$ : the distance from the origin to the point of closest approach in the $x y$ plane.

- $\phi_{0}$ : the azimuthal angle of the track at the point of closest approach.

- $\lambda$ : the dip angle relative to the transverse plane at the point of closest approach.

- $\omega=1 / p_{t}$ : the curvature of the helix.

$d_{0}$ and $\omega$ are variables whose sign depends on the charge of the track. The track finding and fitting procedures use a Kalman fitting algorithm ${ }^{[36]}$ which takes into account the detailed distribution of the material and the magnetic field in the detector. Charged particle tracking has been studied on a wide range of different events: cosmic ray muons, $e^{+} e^{-} \rightarrow\left(e^{+} e^{-}, \mu^{+} \mu^{-}, \tau^{+} \tau^{-}\right.$, and multi-hadron $)$events.

The reconstruction routine utilizes information provided by the L3 trigger and the tracking algorithm. It first improves the estimate of the event start time, $t_{0}$, from a fit to the parameters $d_{0}, \phi_{0}$, and $t_{0}$ based on four-hit track segments in the DCH superlayers. Tracks are then selected by performing helix fits to the track segments found by the L3 track finding algorithm. Further hits in the DCH that might belong to the track are sought. $t_{0}$ is further improved by using only hits associated with tracks.

Two more tracking procedures are applied to find tracks that either do not pass through the entire DCH or do not originate from the IP. These procedures primarily use track segments that have not already been associated with another track. At each iteration, the $t_{0}$ is improved by the increasingly cleaner tracking environment. At the end of this procedure, all tracks are refit with the Kalman filter. 
The tracks found in the DCH are then extrapolated into the SVT. Track segments in the SVT are added to a candidate track, accounting for possible multiple scattering in the intervening material and for changes in the magnetic field. Of the SVT track segments, the ones with the smallest hit residuals and the largest number of SVT layers hit are retained, and the Kalman fit is performed on the combined SVT+DCH hits.

After the first pass of the combined SVT+DCH track finding and fitting, any remaining SVT hits which have not been assigned to a track are analyzed by two supplementary, standalone track finding algorithms. The first one uses matched $\phi$ and $z$ space points from SVT layers 1, 3, \& 5, and any consistent space points from the other two layers. A good track requires a minimum of four space points. This algorithm is efficient on a wide range of $d_{0}$ and $z_{0}$ values. The second algorithm starts with circular trajectories from $\phi$ hits and then adds $z$ hits to form helices.
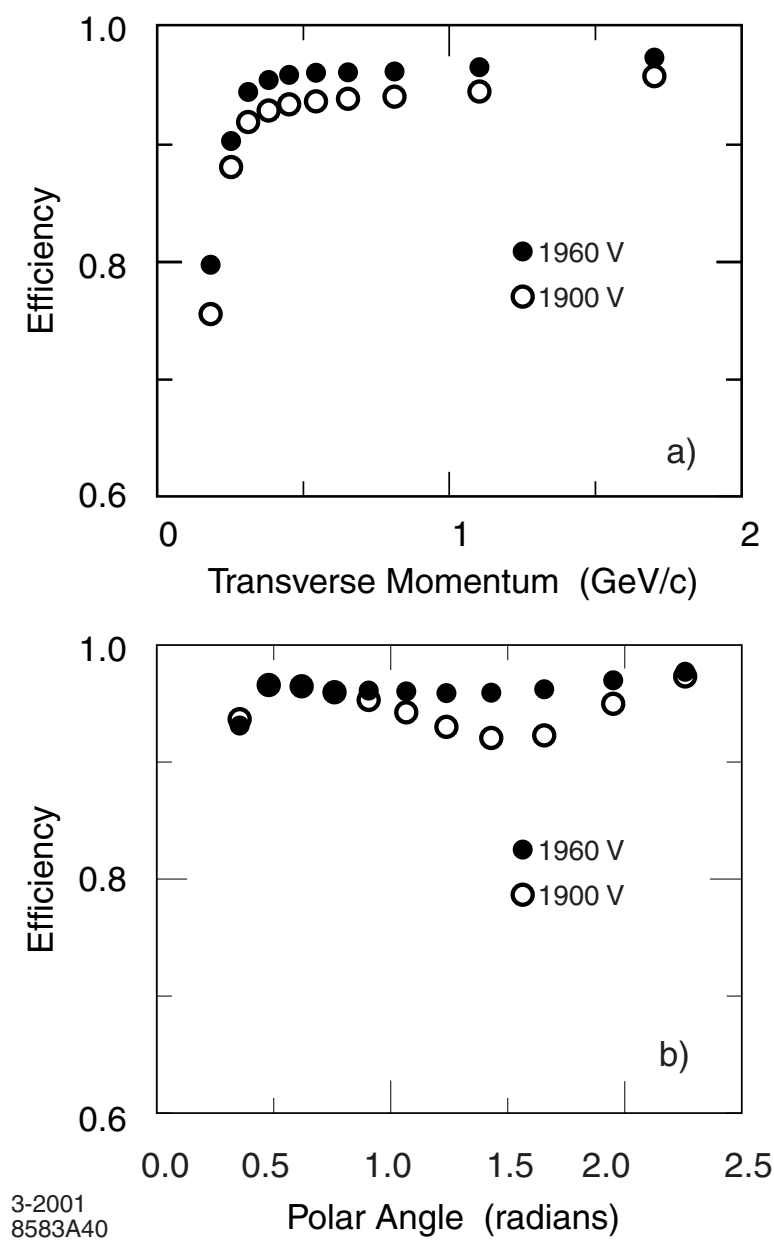

Figure 4.1: Track reconstruction efficiency in the DCH at operating voltages of $1900 \mathrm{~V}$ and $1960 \mathrm{~V}$, as a function of a) transverse momentum, and b) polar angle. The efficiency is measured from multi-hadron events as a fraction of all tracks detected in the SVT for which the DCH portion is also reconstructed. The error bars are smaller than the points shown. 
Finally, an attempt is made to combine tracks found by only one of the two tracking systems, and thus recover tracks scattered in the material between the two detectors.

\subsubsection{Efficiency}

The efficiency for reconstructing tracks in the DCH has been measured as a function of transverse momentum, polar \& azimuthal angles, and track multiplicity for multi-track events. These measurements rely on certain final states and exploit the fact that the track reconstruction can be performed independently in the SVT and DCH.

The absolute tracking efficiency in the DCH is determined by the ratio of the number tracks reconstructed in the DCH to the number in the SVT, provided they fall within the acceptance of the DCH. ${ }^{[29]}$ The efficiency as a function of transverse momentum and polar angle measured from multi-hadron events is shown in Figure 4.1. At the design voltage of $1960 \mathrm{~V}$, the efficiency is $98 \pm 1 \%$ per track above $200 \mathrm{MeV} / c$ and polar angle $\theta>500 \mathrm{mrad}$. At $1900 \mathrm{~V}$, the data shows a drop in efficiency of about $5 \%$ for tracks at close to normal incidence.

For tracks with low transverse momentum, the tracking algorithms for the standalone SVT have a high efficiency. This is particularly important in reconstructing the decay of $D^{*+} \rightarrow D^{0} \pi^{+}$, which is critical to this thesis. Because of the small mass difference between the $D^{*+}$ and the $D^{0}$, the $\pi^{+}$has very little kinetic energy in the $D^{*+}$ rest frame; it is often referred to as the slow pion. To study this efficiency, these decays are selected from data and Monte Carlo samples by reconstructing events of the type $B \rightarrow D^{*} X$ with $D^{*+} \rightarrow D^{0} \pi^{+}, D^{0} \rightarrow K^{-} \pi^{+}$. A comparison between the detected slow pion spectrum in data and Monte Carlo is shown in Figure 4.2. Also shown is the reconstruction efficiency for the slow pion using the MC events. The SVT significantly extends the charged particle tracking capability down to transverse momenta of $\sim 50 \mathrm{MeV} / \mathrm{c}$.

\subsubsection{Track Parameter Resolution}

The resolution of the track parameters is studied using cosmic ray muons which pass near the beam interaction point. Because the tracking system is designed for tracks originating at the IP, the cosmic ray track is split into two segments, one in each of the upper and lower halves of the detector, which are fitted as two separate tracks. To assure that the tracks pass close to the IP, cuts are applied on the $z_{0}, d_{0}$, and $\tan \lambda$. The resolution is derived from the difference of the measured parameters for the two track halves. The results can be seen in Figure 4.3. Based on the full width at half maximum of these distributions the resolutions for single tracks can be stated as

$$
\begin{array}{ll}
\sigma_{d_{0}}=23 \mu \mathrm{m} & \sigma_{\phi_{0}}=0.43 \mathrm{mrad} \\
\sigma_{z_{0}}=29 \mu \mathrm{m} & \sigma_{\tan \lambda}=0.53 \times 10^{-3} .
\end{array}
$$

The transverse momentum dependence of the parameters $z_{0}$ and $d_{0}$ has been measured using multi-hadron events. The resolution is determined from the width of the distribution of the difference between the measured parameters and the coordinates of the vertex 
reconstructed from the remaining tracks in the event. A plot of the distribution is shown in Figure 4.4.

Measurements of the position and angle near the IP are dominated by the SVT measurements. The DCH contributes primarily to the $p_{t}$ measurement. The resolution of $p_{t}$ can be parameterized by the linear function,

$$
\sigma_{p_{t}} / p_{t}=(0.13 \pm 0.01) \% \times p_{t}+(0.45 \pm 0.03) \%,
$$

where the $p_{t}$ is measured in $\mathrm{GeV} / c$.

\subsubsection{Tracking Lists}

The track lists provided by OPR are shown in Table 4.1. The standard list is GoodTracksLoose, which yields good quality tracks with a high efficiency. The requirement of at least $12 \mathrm{DCH}$ hits in this list means that the slow pion from the $D^{*+}$ decay usually does not get included in this list. The GoodTracksVeryLoose list includes the low momentum tracks that are only seen by the SVT.

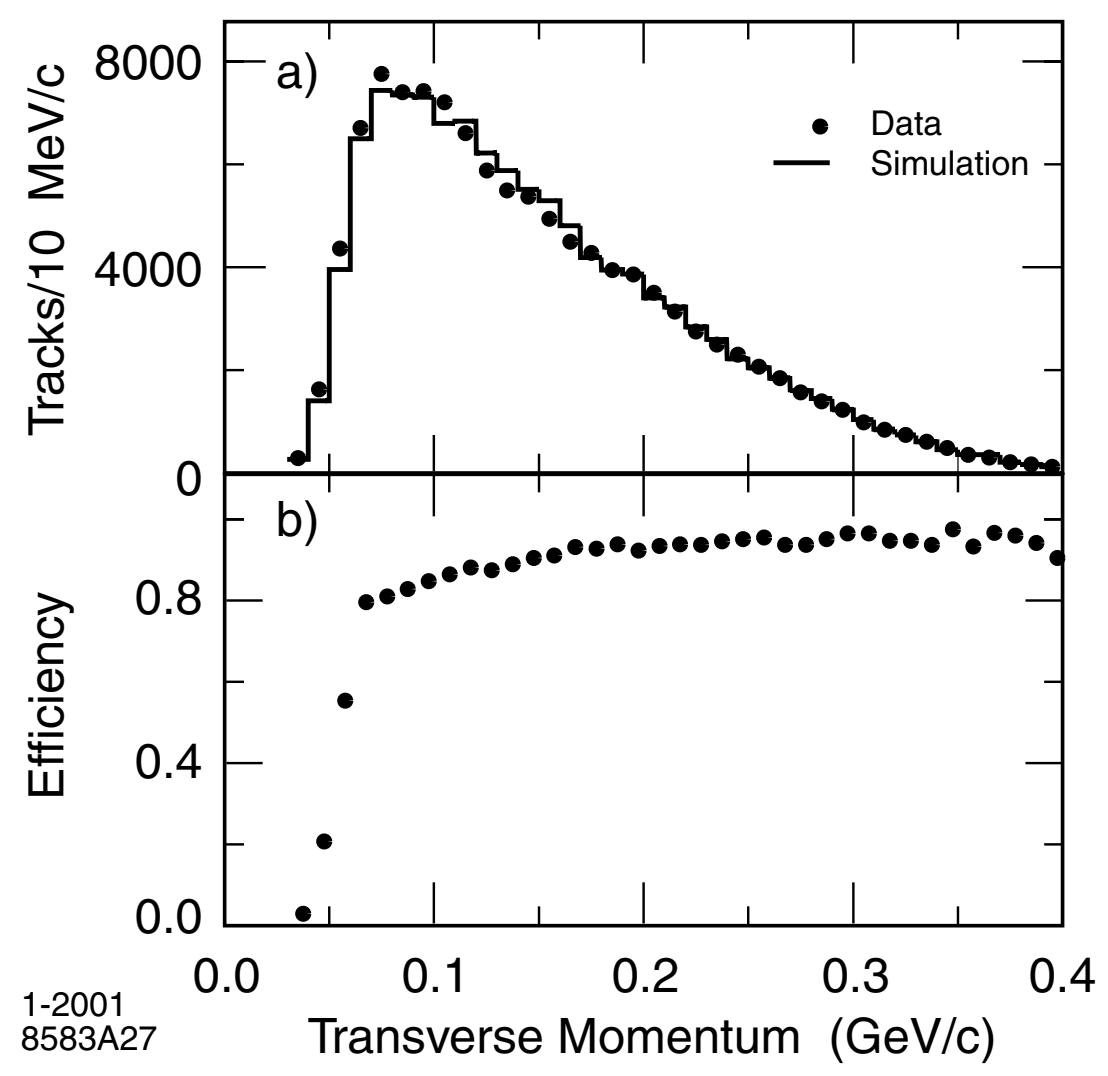

Figure 4.2: Monte Carlo studies of low momentum tracks in the SVT: a) comparison of data (contributions from combinatoric background and non- $B \bar{B}$ events have been subtracted) with simulation of the transverse momentum spectrum of pions from $D^{*+} \rightarrow D^{0} \pi^{+}$in $B \bar{B}$ events, and b) efficiency for slow pion detection derived from simulated events. 


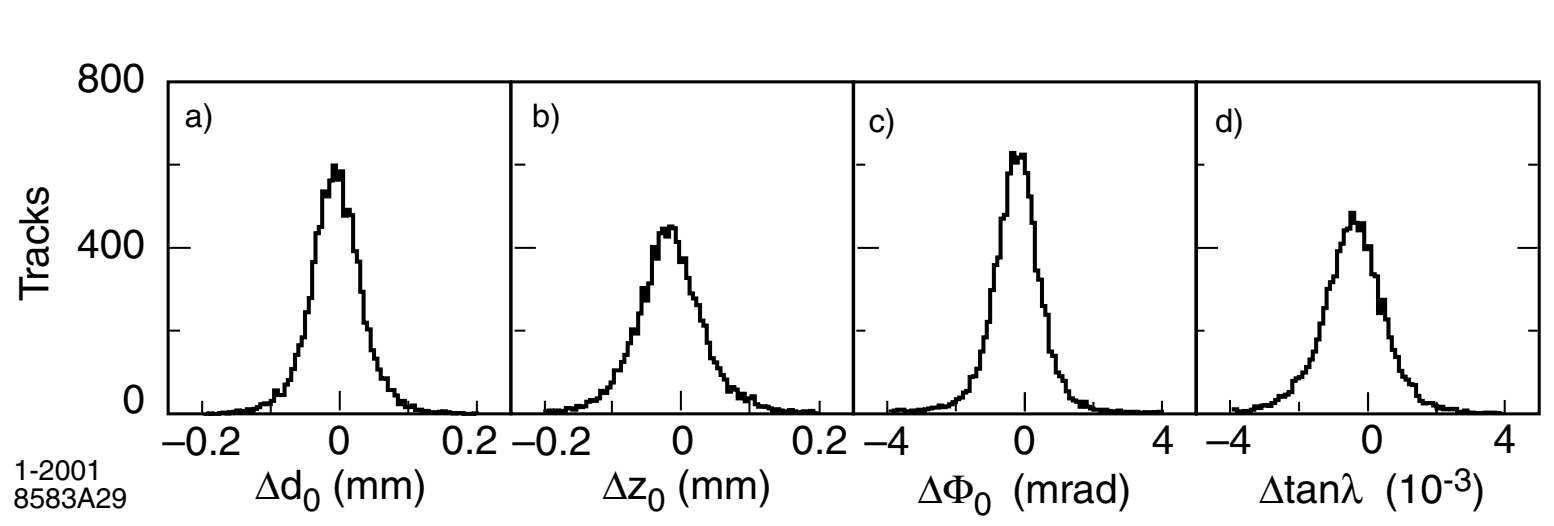

Figure 4.3: Track parameter resolutions based on the differences between the two halves of cosmic ray muon tracks with momenta above $3 \mathrm{GeV} / c$.

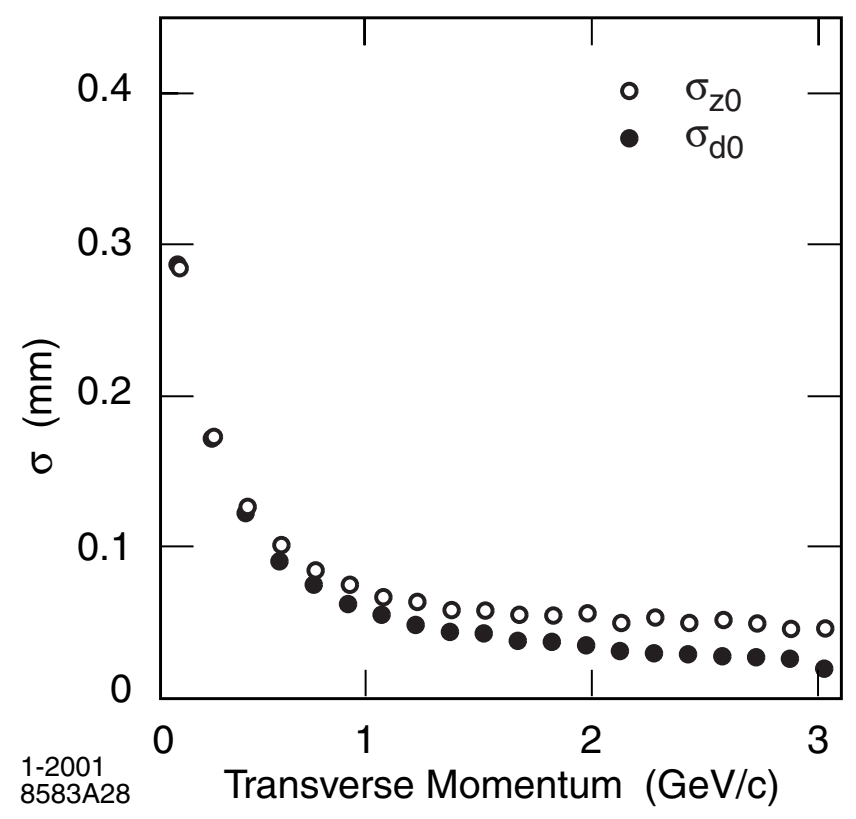

Figure 4.4: Resolutions of track parameters $d_{0}$ and $z_{0}$ as a function of transverse momentum, measured in multi-hadron events. The data are corrected for the effects of particle decays and vertexing errors. The error bars are smaller than the points shown.

\subsection{Particle Identification}

Only five types of charged tracks are sufficiently long-lived to leave a track in the BABAR detector: electrons, muons, pions, kaons, and protons. All other charged particles decay before reaching the first SVT layer. Due to the dominant numbers of pions in multihadron events, for the general reconstruction, all tracks are assumed to be a pion in the initial tracking fits, and the pion mass is used in defining the 4-momentum of the track. Studies have been done to show that this assumption has a negligible effect except in low 


\begin{tabular}{|c|c|}
\hline Track List & Requirements \\
\hline ChargedTracks & $\begin{array}{l}\text { Candidates with non-zero charge, with pion mass hy- } \\
\text { pothesis assigned. }\end{array}$ \\
\hline GoodTracksVeryLoose & $\begin{array}{l}\text { ChargedTracks with: } \\
\text { - Max Momentum: } 10 \mathrm{GeV} / c \\
\text { - Max DOCA in } x y \text { plane: } 1.5 \mathrm{~cm} \\
\text { - Min z DOCA: }-10 \mathrm{~cm} \\
\text { - Max z DOCA: } 10 \mathrm{~cm} \\
\end{array}$ \\
\hline GoodTracksLoose & $\begin{array}{l}\text { Same cuts as GoodTracksVeryLoose with: } \\
\text { - Min Transverse Momentum: } 0.1 \mathrm{GeV} / \mathrm{c} \\
\text { - Min \# of DCH Hits: } 12\end{array}$ \\
\hline GoodTracksAccLoose & $\begin{array}{l}\text { Same as GoodTracksLoose with acceptance cuts: } \\
\text { - Min } \theta: 0.410 \\
\text { - } \operatorname{Max} \theta: 2.54\end{array}$ \\
\hline GoodTracksTight & $\begin{array}{l}\text { Same as GoodTracksLoose, but with these additional } \\
\text { cuts: } \\
\text { - Min \# of DCH Hits: } 20 \\
\text { - Max DOCA in } x y \text { plane: } 1.0 \mathrm{~cm} \\
\text { - Min } z \text { DOCA: }-3.0 \mathrm{~cm} \\
\text { - Max z DOCA: } 3.0 \mathrm{~cm}\end{array}$ \\
\hline
\end{tabular}

Table 4.1: Definitions of track lists produced in OPR. ${ }^{[37]}$

momentum particles, ${ }^{[38]}$ and it is easy to correct for a different particle hypothesis in the later analysis if another choice is needed. Specialized algorithms have been developed by $B A B A R$ which are used to identify electrons, kaons and muons. When particle ID is used to select a track, the mass hypothesis used for that track is changed to that of the selected particle.

\subsubsection{Muon Identification}

The muon identification is based on information from the instrumented flux return, with some information from the calorimeter. ${ }^{[39]}$ A muon is expected to deposit a minimum of energy in the EMC and pass through several layers of steel in the flux return. Several variables are used for muon identification, including:

- The energy deposited in the $\operatorname{EMC}\left(E_{\text {cal }}\right)$.

- The number of IFR hit layers in a cluster $\left(N_{L}\right)$.

- The number of interaction lengths $(\lambda)$ traversed by a track through the whole 
$B A B A R$ detector to the the last IFR layer hit. This is estimated by extrapolation of a track through the IFR.

- The difference $\left(\Delta \lambda=\lambda_{\exp }-\lambda\right)$ between the measured number of interaction lengths and the expected number for a muon hypothesis $\left(\lambda_{\text {exp }}\right)$.

- The average $(\bar{m})$ and standard deviation $\left(\sigma_{m}\right)$ of the multiplicity of hit strips per layer.

- The continuity of the IFR hits $\left(T_{c}\right)$ defined as the fraction of IFR layers hit out of the total number of layers from the first hit layer to the last one.

- The $\chi^{2}$ per degree of freedom of the IFR hit strips in the cluster with respect to the track extrapolation $\left(\chi_{t r k}^{2}\right)$, and with respect to a polynomial fit of the twodimensional IFR cluster $\left(\chi_{\text {fit }}^{2}\right)$.

There are five selection categories for muons: Minimum Ionizing Particle (MIP), VeryLoose, Loose, Tight, and Very-Tight. The criteria are shown in Table 4.2. The first three categories are used for higher muon efficiency, where hadron contamination is less critical. The MIP category is $99 \%$ efficient for muons, but also accepts over $60 \%$ of pions and kaons. The Tight and Very-Tight categories are designed to minimize misidentification, but at a cost in muon efficiency. The Very-Tight selector has an efficiency of $66 \%$ for muons, with only a $1.9 \%$ misidentification of pions. The Very-Tight selector is used in this thesis.

\begin{tabular}{|l|l|l|l|l|}
\hline $\begin{array}{l}\text { Minimum Ioniz- } \\
\text { ing Particle }\end{array}$ & Very-Loose & Loose & Tight & Very-Tight \\
\hline$E_{\text {cal }}<0.5(\mathrm{GeV})^{\mathrm{a}}$ & $E_{\text {cal }}<0.5^{\mathrm{a}}$ & $E_{\text {cal }}<0.5^{\mathrm{a}}$ & $0.05<E_{\text {cal }}<0.4^{\mathrm{b}}$ & $0.05<E_{\text {cal }}<0.4^{\mathrm{b}}$ \\
& $N_{L} \geq 2$ & $N_{L} \geq 2$ & $N_{L} \geq 2$ & $N_{L} \geq 2$ \\
& $\Delta \lambda<2.5$ & $\Delta \lambda<2$ & $\Delta \lambda<1$ & $\Delta \lambda<0.8$ \\
& $\lambda>2$ & $\lambda>2$ & $\lambda>2.2$ & $\lambda>2.2$ \\
& $T_{c}>0.1^{\mathrm{c}}$ & $T_{c}>0.2^{\mathrm{c}}$ & $T_{c}>0.3^{\mathrm{c}}$ & $T_{c}>0.34^{\mathrm{c}}$ \\
& $\bar{m}<10$ & $\bar{m}<10$ & $\bar{m}<8$ & $\bar{m}<8$ \\
& $\sigma_{m}<6$ & $\sigma_{m}<6$ & $\sigma_{m}<4$ & $\sigma_{m}<4$ \\
& & $\chi_{\text {trk }}^{2}<7$ & $\chi_{\text {trk }}^{2}<5$ & $\chi_{\text {trk }}^{2}<5$ \\
& & $\chi_{\text {fit }}^{2}<4$ & $\chi_{\text {fit }}^{2}<3$ & $\chi_{\text {fit }}^{2}<3$ \\
\hline
\end{tabular}

a Applied only on tracks having a cluster in the EMC.

b Applied on tracks in the angular region covered by the EMC: $\theta(\mathrm{rad})<2.45$.

c Applied only on tracks in the polar angle interval $0.3<\theta(\mathrm{rad})<1$.

Table 4.2: Criteria for the different categories in the muon ID system. 


\subsubsection{Charged Kaon Identification}

The kaon selection used in this analysis is likelihood based. ${ }^{[40]}$ The selectors use the ionization energy loss, $\mathrm{d} E / \mathrm{d} x$, in the SVT and DCH, the Cherenkov angle, $\theta_{C}$, and the number of photons in the DIRC. The kaon ID information from each detector is limited to particular momentum ranges. $\mathrm{d} E / \mathrm{d} x$ information provides kaon separation below 700 $\mathrm{MeV} / c$, and again above $1.5 \mathrm{GeV} / c$ due to the relativistic rise for pions. The DIRC provides $\pi / K$ separation above $600 \mathrm{MeV} / c$.

The $\mathrm{d} E / \mathrm{d} x$ values from both the SVT and DCH are parameterized for the different long-lived particles types $(e, \mu, \pi, K, p)$ as a function of momentum based on the Bethe-Bloch formula. The probability density function (PDF) for both detectors is a Gaussian distribution, with the mean given by the $\mathrm{d} E / \mathrm{d} x$ calibration. The Bethe-Bloche parameters and the $\mathrm{d} E / \mathrm{d} x$ resolution are calibrated by measuring the average charge deposited by a minimum ionizing particle for each cell. The relative $\mathrm{d} E / \mathrm{d} x$ error for the parameterization is $10 \%$ for the SVT and $8 \%$ for the DCH.

The likelihood function for the DIRC is based on two parts: the Cherenkov angle, and the number of photons detected. These are assumed to be uncorrelated. The PDF based on the Cherenkov angle is a Gaussian distribution. It uses the values of $\theta_{C}$ from Eq. 3.2, the associated error, and the expected angle for the particle hypotheses $(e, \mu, \pi, K, p)$. The five Gaussian distributions are normalized such that their sum equals 1.0. For particle hypotheses where the momentum is below the Cherenkov threshold, a probability of $\frac{1}{5}$ is

\begin{tabular}{|c|c|c|}
\hline & Loose & Tight \\
\hline SVT $(\mathrm{GeV} / c)$ & $p<0.6, \quad p>1.5$ & $p<0.7$ \\
\hline $\mathrm{DCH}(\mathrm{GeV} / c)$ & $p<0.6, \quad p>1.5$ & $p<0.7$ \\
\hline DIRC $(\mathrm{GeV} / c)$ & $p>0.6$ & $p>0.6$ \\
\hline Likelihood & $l_{K} / l_{p} \geq 1 ; \quad l_{K} / l_{\pi}>r_{\pi}$ & $l_{K} / l_{p}>1 ; \quad l_{K} / l_{\pi}>r_{\pi}$ \\
\hline \multirow[t]{3}{*}{ Requirements } & $p<2.7 \mathrm{GeV} / c:$ & $p>2.7 \mathrm{GeV} / c:$ \\
\hline & $p>2.7 \mathrm{GeV} / c:$ & $p>2.7 \mathrm{GeV} / c:$ \\
\hline & $0.5<p<0.7 \mathrm{GeV} / c: \quad r_{\pi}=15$ & $0.5<p<0.7 \mathrm{GeV} / c: \quad r_{\pi}=15$ \\
\hline & " Very-Tight & "Not-a-Pion \\
\hline$\overline{\mathrm{SVT}}(\mathrm{GeV} / \mathrm{c})$ & $p<0.6$ & $p<0.5$ \\
\hline $\mathrm{DCH}(\mathrm{GeV} / c)$ & $p<0.6$ & $p<0.6$ \\
\hline $\operatorname{DIRC}(\mathrm{GeV} / c)$ & $p>0.6$ & $p>0.6$ \\
\hline Likelihood & $l_{K} / l_{p}>1 ; \quad l_{K} / l_{\pi}>r_{\pi}$ & \multirow{4}{*}{$\begin{array}{l}\text { Default }=\text { true. } \\
l_{p} / l_{\pi}<r_{\pi} \quad \& \quad l_{K} / l_{\pi}<r_{\pi} \\
p \leq 0.5 \mathrm{GeV} / c: \quad r_{\pi}=0.1 \\
p>0.5 \mathrm{GeV} / c: \quad r_{\pi}=1.0\end{array}$} \\
\hline Requirements & $p<2.5 \mathrm{GeV} / c:$ & \\
\hline & $p>2.5 \mathrm{GeV} / c$ & \\
\hline & $0.4<p<0.7 \mathrm{GeV} / c:$ & \\
\hline
\end{tabular}

Table 4.3: Kaon ID selection criteria. The momentum cuts to include the likelihood from each detector are also shown. $l_{i}$ is the likelihood for particle type $i$. The value $r_{\pi}$ represents the ratio of likelihood values. It used used as the threshold for selecting the kaon at specific momenta. 
assigned. The PDF based on the number of photons detected is a Poisson probability:

$$
p_{i}=\operatorname{Poisson}\left(N_{s}+N_{b}, N_{e x p}^{i}+N_{b}\right), \quad i=(e, \mu, \pi, K, p)
$$

where $N_{s}$ is the number of detected (signal) photons, $N_{b}$ is the number of background (fixed at 3), and $N_{e x p}^{i}$ is the expected number of photons, and the Poisson distribution is

$$
\operatorname{Poisson}(n, x)=\frac{x^{n} e^{-x}}{n !}
$$

which is normalized to a sum of one. The total probability is the product of the two PDFs, again normalized to an overall sum of one.

There are four main selection categories for kaon identification: Not-a-Pion, Loose, Tight, \& Very-Tight. The selection criteria are shown in Table 4.3. The Not-a-Pion category is aimed at simply rejecting pions in order to optimize the efficiency for kaons. The efficiency of the Not-a-Pion selector is 97\%, but with a $\sim 11 \%$ acceptance of pions. Other aspects of the $D^{*}$ reconstruction are very effective at reducing the pion background, so tighter kaon selection is not necessary. The Not-a-Pion category is the only kaon selector used in this thesis.

\subsubsection{Electron Identification}

Since only the $\bar{B}^{0} \rightarrow D^{*+} \mu^{-} \bar{\nu}_{\mu}$ decay is being studied in this analysis, electron identification is not used. However, the electron mode is very important in other $\bar{B}^{0} \rightarrow D^{*+} \ell^{-} \bar{\nu}_{\ell}$ analyses, so a brief description of the electron ID strategy is provided.

The main properties used in electron identification are the shower energy and lateral shape in the EMC. ${ }^{41]}$ As was described in Section 3.6, the $E / p$ ratio is near 1 for electrons and is the primary selection variable. Electromagnetic showers in the EMC tend to be narrower and more regular than hadronic showers, so the shape of the shower is used to distinguish between hadrons and electrons. Also, the $\mathrm{d} E / \mathrm{d} x$ in the DCH must be consistent with that of an electron.

As with the other PID algorithms, several levels of electron selector are defined. The two most common are the Loose and the Very-Tight selectors. The Loose selector has an efficiency greater than $98 \%$ for electrons with momentum above $1 \mathrm{GeV} / c$. The efficiency for the Very-Tight selector is $90 \%$ with a misidentification probability of only $0.2 \%$. Thus, the electron selector has both a high efficiency and a high hadron rejection.

\subsubsection{PID Control Samples}

In order to properly measure the efficiency of any particle identification algorithm, pure samples of the different particle species are required. The samples must be obtained without using any PID algorithms; otherwise, the efficiencies would be biased. Certain specific events can be used to obtain control samples.

The control sample for electrons is based on radiative Bhabha scattering, where one

or both of the electrons loses energy through bremsstrahlung or initial or final state 
radiation. The radiated photon allows for the electron energies to be distributed over the whole phase space of interest to BABAR.

Control samples for hadrons are obtained by reconstructing certain specific decays. The hadron in question is identifiable among the decay products. The kaon control sample is identified from the decay of $D^{*} \rightarrow D^{0} \pi, D^{0} \rightarrow K \pi$. The kaon in the $D^{0}$ decay can be cleanly isolated in the reconstruction of this decay. The pion in the $D^{0}$ decay can also be isolated into a control sample in the same reconstruction. Another control sample for pions uses the decay $K_{S}^{0} \rightarrow \pi^{+} \pi^{-}$. The proton control sample is obtained from the decay, $\Lambda \rightarrow p \pi$.

There are two control samples for muons, both of which are obtained from the primary $e^{+} e^{-}$collision without subsequent decays involved. One is from the interaction $e^{+} e^{-} \rightarrow$ $e^{+} e^{-} \mu^{+} \mu^{-}$. This is an electron scattering event in which a pair of muons are produced from the virtual photon. The other type of control sample comes from radiative muon pair production $e^{+} e^{-} \rightarrow \mu^{+} \mu^{-} \gamma$. The photon comes from initial or final state radiation of one of the muons. My major contribution to BABAR central physics was the production and analysis of the $\mu^{+} \mu^{-} \gamma$ control sample. ${ }^{[42]}$ This included the production of muon ID efficiency tables based on this control sample. These are used throughout the BABAR collaboration for analyses involving muons. The document written for the work on this control sample is provided in Appendix A. 


\section{Chapter 5}

\section{Data and Monte Carlo Selection}

This chapter provides a description of the data and Monte Carlo simulated data used in this analysis, and the criteria for selecting the $\bar{B}^{0} \rightarrow D^{*+} \mu^{-} \bar{\nu}_{\mu}$ events. Sections 5.1 and 5.2 describe the data and Monte Carlo ${ }^{\mathrm{a}}$ respectively. Section 5.3 describes one of the significant backgrounds in the BABAR experiment. Section 5.4 provides the details of the cuts and selection criteria for isolating a clean sample of $\bar{B}^{0} \rightarrow D^{*+} \mu^{-} \bar{\nu}_{\mu}$ decays in order to determine the branching fraction for this decay. The methods described in this chapter are the standard BABAR techniques for reconstructing $\bar{B}^{0} \rightarrow D^{*+} \ell^{-} \bar{\nu}_{\ell}$ decays which are used in many types of analyses including $B^{0}$ mixing and lifetime studies, $\left|V_{c b}\right|$ measurement, partial reconstruction and identification of $B$ mesons for aspects of $\mathrm{CP}$ violation measurements and very rare $B$ decay studies. After all selection criteria are applied, several corrections are made to the Monte Carlo samples to provide better modeling of the data. These corrections are described in section 5.5.

\subsection{BABAR Data}

This analysis uses the data acquired by the BABAR detector during the October 1999 to August 2000 running period, referred to as Run 1. This corresponds to $20.6 \mathrm{fb}^{-1}$ collected at the $\Upsilon(4 S)$ resonance peak, and $2.6 \mathrm{fb}^{-1}$ collected at $40 \mathrm{MeV}$ below the peak. The total number of $B \bar{B}$ pairs analyzed is estimated to be 22.3 million (see Section 3.2).

The decay chain being analyzed in this thesis is $\bar{B}^{0} \rightarrow D^{*+} \mu^{-} \bar{\nu}_{\mu}$ with subsequent decays of $D^{*+} \rightarrow D^{0} \pi^{+}$and $D^{0} \rightarrow K^{-} \pi^{+}$(and charge conjugate modes). The code to isolate a reasonably pure sample of this decay was written by the $D^{*} \ell \nu$ working group at BABAR. It is designed to isolate both the muon and electron modes as well as several different $D^{0}$ decay modes: $D^{0} \rightarrow K^{-} \pi^{+}, K^{-} \pi^{+} \pi^{-} \pi^{+}, K^{-} \pi^{+} \pi^{0}, K_{S}^{0} \pi^{+} \pi^{-}$. The single mode of $D^{0} \rightarrow K^{-} \pi^{+}$was chosen because it provides the cleanest signal and lowest systematic error in the reconstruction. In other analyses in which more events are required, the other decay modes provide a significant increase in reconstructed decays without requiring higher integrated luminosity from the detector.

At the beginning of this analysis, Run 2 data collection was not complete, and that data was not fully processed through OPR. Thus, only the Run 1 data was chosen. Using more data as it became available was considered, but the benefit of the extra data had little impact on the precision of this measurement due to systematic errors. Since the systematic error is relatively large, the important consideration was in choosing a

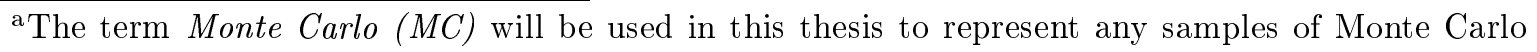
simulated data.
} 
clean signal over high statistics. At this time, additional data from Run 2 (2001) and Run 3 (2002) could provide almost four times as many $B \bar{B}$ decays, but the additional data does not improve the systematic errors. ${ }^{[10]}$ Additionally, the gradual loss in IFR efficiency, as described in Section 3.8.1, would result in an increase in the systematic error in muon identification efficiency. Thus, the trade-offs for including the newer data are not compelling.

In order to reduce the computational load from hundreds of physicists analyzing the same full data set, numerous skims have been created for the data. During OPR processing, special boolean variables (tags) have been created to label each event as potentially containing certain classes of decays. These tags are designed to have a very high efficiency for the real signal events - as well as a high background rate - but events which clearly are another type of decay are eliminated. So, in many analyses, instead of analyzing the full data set, only the appropriate skim data set is needed. This saves considerable time in not having to process events which would simply be rejected by the preliminary cuts anyway.

Since the $D^{*+} \ell^{-} \bar{\nu}_{\ell}$ skim was used in this analysis, some cuts have already been applied before plots could be made to show their effectiveness. Therefore, plots of certain cuts will be taken from another BABAR analysis document ${ }^{[44]}$ which shows the cuts and their implications in more detail for the $D^{*+} \ell^{-} \bar{\nu}_{\ell}$ reconstruction.

\subsection{Monte Carlo Simulated Data}

In order to effectively understand the backgrounds, the data are simulated using the GEANT 4 Monte Carlo software. ${ }^{[4]}$ The Monte Carlo data set is divided into two main categories. Single-mode MC samples are collections of specific decays, such as the decay chain being studied here. Single-mode MC is very useful for studying specific decays in pure form, either as signal, or as a pure source of background. Single-mode MC samples have been generated for many decays of interest by the BABAR collaborators. Generic MC samples try to model the data as a whole. For each particle in a sample, all known decay modes are modeled with the branching fractions and spectra specified as well as they are known at the time. For the MC samples used in this analysis, the decays and the associated branching fractions are generally taken from the world averages assembled and calculated by the the Particle Data Group in the year 2000, ${ }^{[46]}$ although some are taken from theoretical models as well. Corrections are also applied later in the analysis where the simulation is known to disagree with the measured data. Generic samples have been generated for $B^{0} \bar{B}^{0}$ and $B^{+} B^{-}$mesons as well as for events of the types $e^{+} e^{-} \rightarrow$ $\left(c \bar{c}, s \bar{s}, u \bar{u}, d \bar{d}, e^{+} e^{-}, \mu^{+} \mu^{-}\right.$, and $\left.\tau^{+} \tau^{-}\right)$.

The MC samples are generated so as to produce exactly the same type of raw signals within the detector as real data. These are then processed through OPR exactly as though they are real data. The combination of these samples, scaled to the appropriate luminosities, provides a model of the data produced within the BABAR detector. Because the events are simulated, it is possible to know exactly what decays happened in each event. This information is referred as Monte Carlo truth. By using the truth to identify 


\begin{tabular}{|l|c|c|c|}
\hline Sample & $\begin{array}{c}\text { Number } \\
\text { of events }\end{array}$ & $\begin{array}{c}\text { Cross section } \\
(\mathrm{nb})\end{array}$ & $\begin{array}{c}\text { Equivalent } \\
\text { luminosity }\left(\mathrm{fb}^{-1}\right)\end{array}$ \\
\hline Generic & $29,461,186$ & 0.525 & 56.1 \\
\hline$e^{+} e^{-} \rightarrow \Upsilon(4 S) \rightarrow B^{0} \bar{B}^{0}$ & $30,748,192$ & 0.525 & 58.6 \\
$e^{+} e^{-} \rightarrow \Upsilon(4 S) \rightarrow B^{+} B^{-}$ & $41,332,550$ & 1.30 & 31.8 \\
$e^{+} e^{-} \rightarrow c \bar{c}$ & & & \\
\hline Single-mode & & $\sim 0.0013$ & $\sim 49$ \\
\hline $\bar{B}^{0} \rightarrow D^{*+} \ell^{-} \bar{\nu}_{\ell}$ & $\left(D^{*+} \rightarrow D^{0} \pi^{+}, D^{0} \rightarrow K^{-} \pi^{+}\right)$ & 62,000 & $\sim 0$ \\
\hline
\end{tabular}

Table 5.1: MC samples used in this analysis. All samples listed are modeled with the detector conditions during the Run 1 period. The cross sections listed are at the $\Upsilon(4 S)$ resonance energy for the BABAR experiment. Only an approximate cross section is listed for the $\bar{B}^{0} \rightarrow D^{*+} \ell^{-} \bar{\nu}_{\ell}$ signal MC since that is the quantity under investigation.

different types of backgrounds and signals within a sample after reconstruction, it is possible to obtain a good idea of the composition of the actual data sample and extract the desired information from the data.

Table 5.1 shows a list of the generic and single-mode MC samples used in this analysis and the number of events generated for each sample. Generic MC based on events of type $u \bar{u}, d \bar{d}$, and $s \bar{s}$ are examined, but these events are virtually eliminated by the selection criteria during the $\bar{B}^{0} \rightarrow D^{*+} \ell^{-} \bar{\nu}_{\ell}$ reconstruction. From $27 \mathrm{fb}^{-1}$ of $u \bar{u}, d \bar{d}$, and $s \bar{s} \mathrm{MC}$, only 21 events pass the cuts. This level of background is completely insignificant and not studied further in this analysis.

\subsection{Continuum Background}

A very significant source of potential background comes from decays of $e^{+} e^{-} \rightarrow q \bar{q}$ other than to $b \bar{b}$. This type of background is referred to as continuum background. ${ }^{b}$ As can be seen from Table 5.1, the cross section for $c \bar{c}$ is significant compared to the $\Upsilon(4 S)$ cross section. Fortunately, well chosen cuts can greatly reduce the contribution of continuum events in the final data set. However, they will not be completely eliminated.

In order to help characterize the continuum background, the PEP-II $B$ Factory runs approximately one-eighth of the time at $40 \mathrm{MeV}$ below the $\Upsilon(4 S)$ peak. This off-peak data is below the energy threshold for the creation of $B \bar{B}$ mesons, and therefore makes a very good sample for studying non- $B \bar{B}$ events in the data set.

In the case of this analysis, the continuum background suppression is strong enough to eliminate most of the events in the off-peak data set, and the number of events passing is too few to provide adequate statistics in characterizing the continuum background. In

\footnotetext{
${ }^{\mathrm{b}}$ Continuum background describes any event of the type $e^{+} e^{-} \rightarrow$ (anything except $b \bar{b}$ ). Only $e^{+} e^{-}$ $\rightarrow c \bar{c}$ events provide a significant contribution to the continuum background for this analysis.
} 


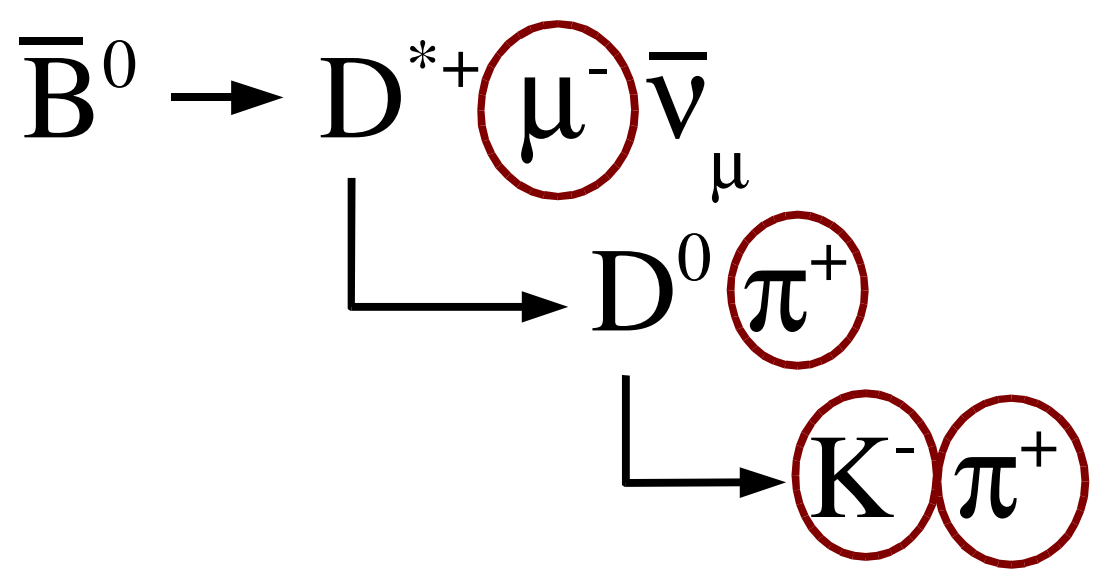

Figure 5.1: The decay chain reconstructed in this analysis. The circled particles are seen as tracks in the detector. The $B$ and $D$ mesons decay too quickly to leave any tracks, and neutrinos are undetectable by BABAR.

fact, of the $2.6 \mathrm{fb}^{-1}$ of off-peak data, only four events pass all of the cuts described below. Therefore, due to the extremely low statistics, the off-peak data from Run 1 are not used. Instead, the generic MC of $c \bar{c}$ events is used because it simulates a much larger set of continuum data.

\subsection{Event Reconstruction and Selection}

The basic reconstruction technique is to combine the tracks seen by the detector into composites of the shorter lived particles. When two or more tracks meet at a vertex, their 4-vectors are combined. If the tracks originated from the decay of another particle, the invariant mass of the combined 4 -vectors should be that of the decayed particle. This reconstructed particle is tracked back to see if it meets any further particles.

The decays reconstructed in this analysis are shown in Figure 5.1. A $K^{-}$is combined with a $\pi^{+}$to form a $D^{0}$. This is then combined with another $\pi^{+}$to form the $D^{*+}$, which is further combined with a lepton. Unfortunately, since the neutrino is undetectable, it is not possible to fully reconstruct the $\bar{B}^{0}$ meson in the decay. Instead, the combined $D^{*+} \ell^{-}$ is used as a good approximation to the $\bar{B}^{0}$ meson. At each level of the reconstruction, a number of cuts are applied to the tracks or the vertex to ensure that the reconstruction is done correctly and that background events are rejected. The rest of this chapter will detail each level of the reconstruction and selection criteria.

\subsection{1 $\quad D^{0}$ Reconstruction}

The $D^{0}$ is reconstructed from a $K^{-}$and a $\pi^{+}$. Both of these tracks are taken from the GoodTracksLoose list (see Section 4.1.4). The kaon must pass the Not-a-Pion kaon ID selector. The $D^{0}$ mass peak obtained from 4 -vector addition is shown in Figure 5.2. The Gaussian width of the peak is about $7 \mathrm{MeV} / c^{2}$. To select the $D^{0}$ candidates used in this 


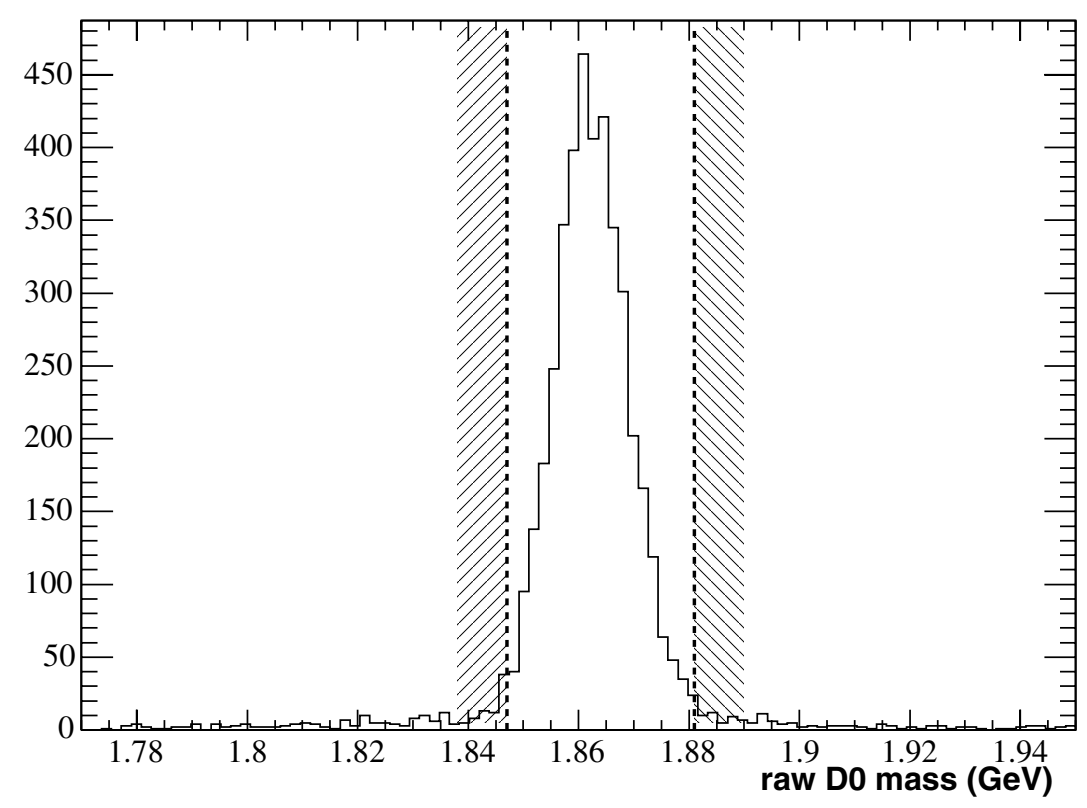

Figure 5.2: Raw $D^{0}$ candidate mass distribution taken from data. The cuts applied are shown. In this and subsequent plots, the hash marks on the cut line indicate the data removed by the cut.

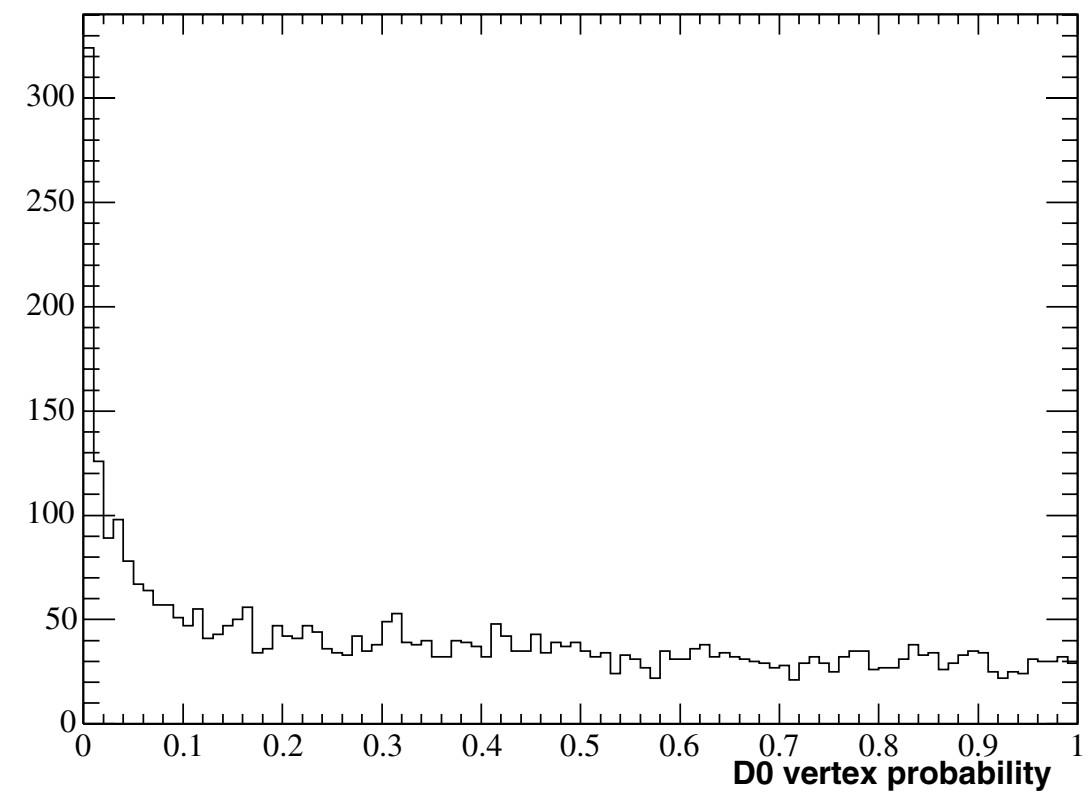

Figure 5.3: $\chi^{2}$ probability of $D^{0}$ mass-constrained fit taken from data. The cut at 0.001 is at too small a value to show on the plot. 
analysis, a cut is made at 2.5 times the width, or $\pm 17 \mathrm{MeV} / c^{2}$ from the nominal $D^{0}$ mass of $1.8646 \mathrm{GeV} / c^{2}{ }^{26]}$

The vertex is fitted with the invariant mass constrained to that of the nominal $D^{0}$ mass. A cut is applied on the $\chi^{2}$ probability of the fit at 0.001 . This value is based on the $\chi^{2}$ per degree of freedom of the fit. For tracks which actually come from the vertex, their normal statistical distribution will produce a $\chi^{2}$ probability which is flat over the full range of $[0,1]$. For random tracks which happen to cross, the $\chi^{2}$ probability is very sharply peaked at zero, as show in Figure 5.3. A cut at 0.001 retains $99.9 \%$ of the real $D^{0}$ s s while removing a significant amount of background, as measured with data.

\subsection{2 $D^{*+}$ Reconstruction}

The mass difference between the $D^{*+}$ and the $D^{0}$ is only slightly larger than the pion mass. This small mass difference gives very little phase space for the slow pion. This means that the spectrum of this difference is a well defined peak, and provides a means to improve the reconstruction, and substantially reduce the background from mis-reconstructed $D^{*}$ 's. Unfortunately, the low energy of the pion means that it often does not reach the drift chamber, and is therefore more difficult to track accurately. The fact that the $\Upsilon(4 S) \mathrm{CM}$ frame is boosted (see Section 3.1) does not help to alleviate this problem because the boost is along the axial direction of the detector.

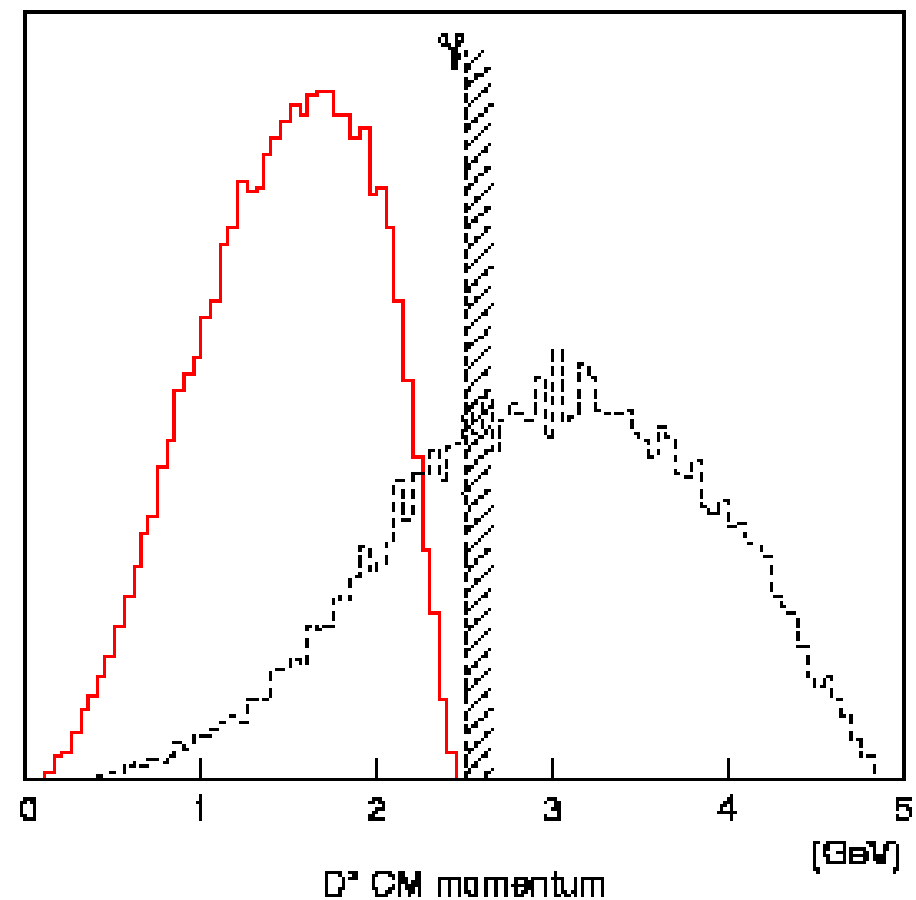

Figure 5.4: $D^{*+}$ momentum spectra in the CM frame for signal events (solid line) and continuum events (dashed line), both taken from MC. This plot and Figure 5.7 are taken from reference 44 , and the image quality is poor in the original document. 


\section{SVT}

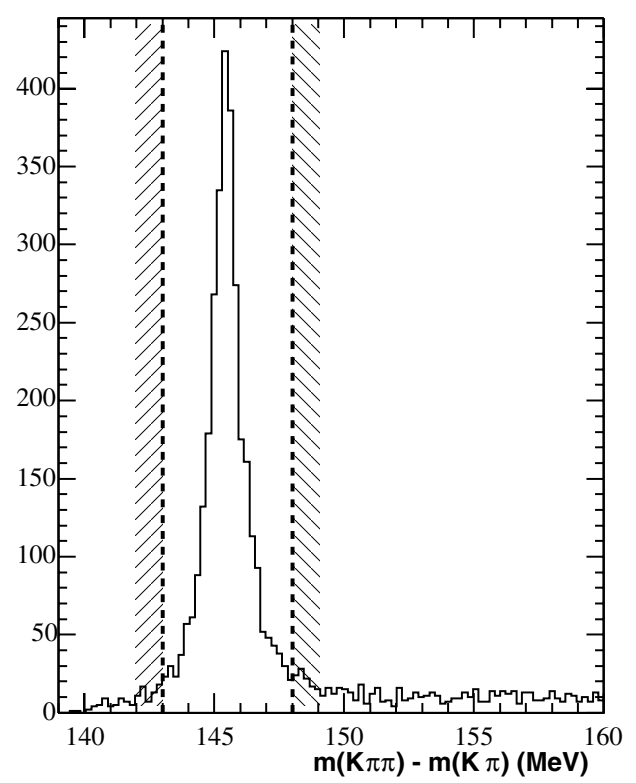

\section{SVT+DCH}

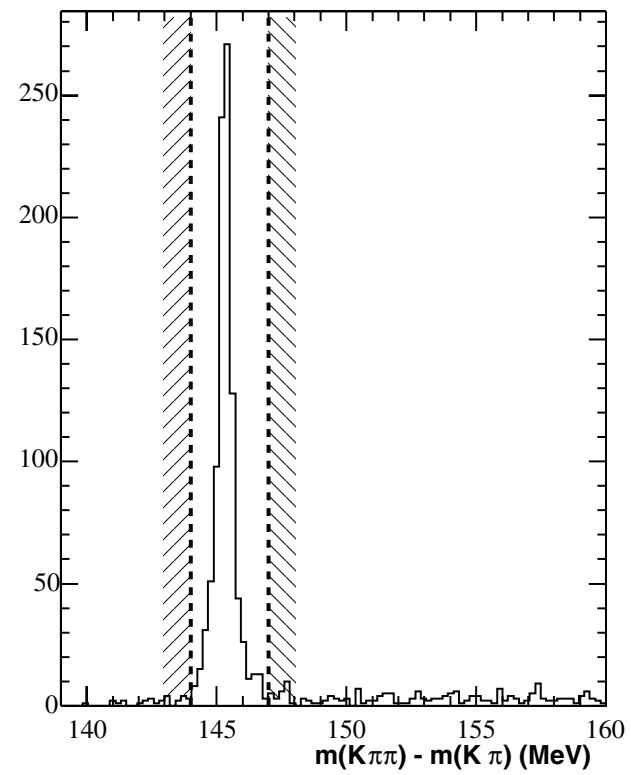

Figure 5.5: $\delta m$ for slow pion in SVT or SVT+DCH, both taken from data. Cuts are dependent upon whether or not the slow pion enters the DCH.

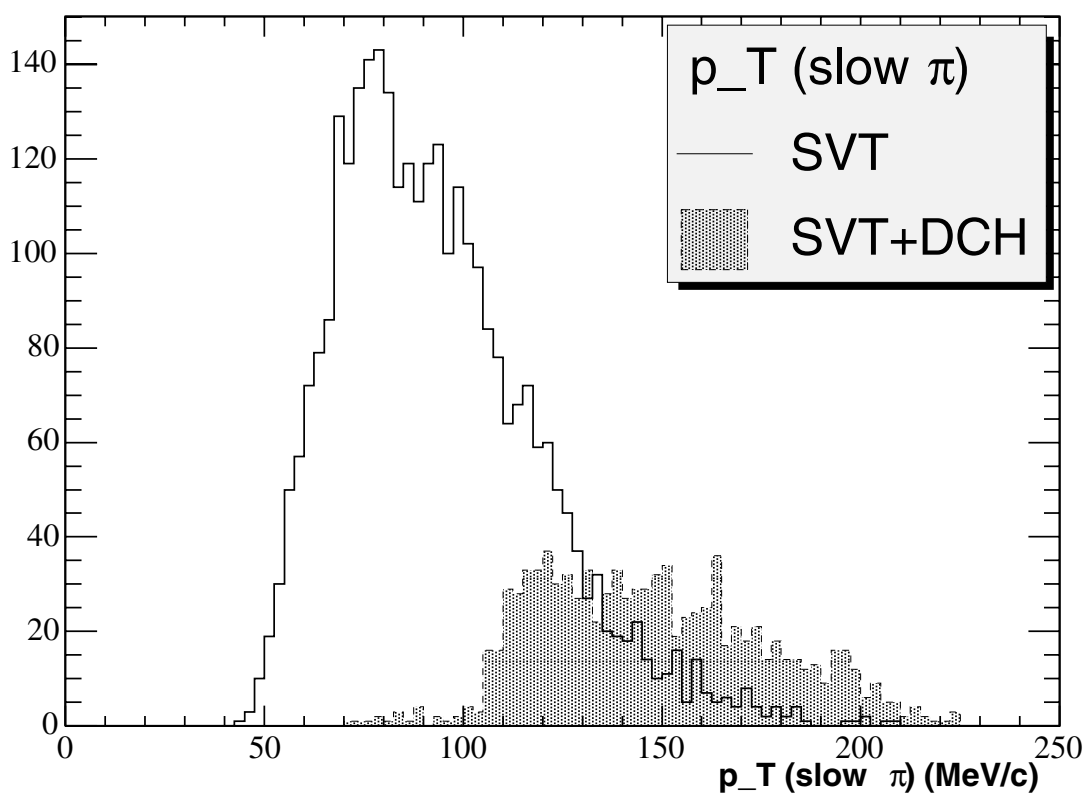

Figure 5.6: Transverse momentum of slow pion in SVT or SVT+DCH taken from data. The majority of the slow pions fail to reach the $\mathrm{DCH}$. 
The $D^{0}$ candidate is combined with a low momentum pion from the GoodTracksVeryLoose list to make the $D^{*+}$ candidate. The slow pion must have a total momentum less than $450 \mathrm{MeV} / c$. The transverse momentum must be greater than $50 \mathrm{MeV} / c$ in order for it to be tracked in the SVT. Furthermore, its charge must be opposite that of the kaon in order for both particles to be daughters of the same $D^{*+}$ decay. Because the $D^{*+}$ is required to come from a $B$ meson decay, the $D^{*+}$ momentum in the CM frame must be less than $2.5 \mathrm{GeV} / c$. Figure 5.4 shows the effect of this cut in separating $D^{*+}$ mesons from $B$ decays versus $D^{*}$ 's from the continuum.

The combinatoric background (other hadrons mis-reconstructed as $D^{*+}$ mesons, see Section 6.7) in the $D^{*+}$ reconstruction can be strongly reduced using a cut on the mass difference between the reconstructed $D^{*+}\left(K^{-} \pi^{+} \pi^{+}\right)$and the $D^{0}\left(K^{-} \pi^{+}\right)$. This quantity is sharply peaked, producing a high signal-to-noise ratio, as can be seen in Figure 5.5. The width of this peak is dependent on whether or not the slow pion is tracked in the $\mathrm{DCH}$. If the slow pion does enter the DCH, it is tracked with a much higher resolution, and the width of the $\delta m$ peak becomes correspondingly narrower. However, as is shown in Figure 5.6, most of the slow pions fail to reach the DCH. In order to accommodate the improved resolution from the DCH, the cuts are conditional. For events in which the slow pion remains in the SVT only, the requirements are $143<p_{T}\left(\pi_{\text {slow }}\right)<148 \mathrm{MeV} / c$. If the slow pion reaches the $\mathrm{DCH}$, the cuts are tightened to $144<p_{T}\left(\pi_{\text {slow }}\right)<147 \mathrm{MeV} / c$.

Because the $\delta m$ spectrum is well defined, it can also be used in characterizing the combinatoric background which passes this cut. As will be described in the next chapter, the spectrum can be fitted for PDFs describing the signal and background. From these fits on the data, the amount of combinatoric background can be determined without relying on the MC modeling.

\subsection{3 $D^{*} \ell$ Reconstruction}

The $D^{*+}$ candidate is combined with a track from the GoodTracksTight list which has also passed the Very-Tight muon (or electron) selector. Given that the muon came directly from the $B$ decay, it would have a high momentum in the CM frame, so a cut is applied at $p_{\ell}^{*}>1.2 \mathrm{GeV} / c$. This helps to reduce the background of leptons from decays other than $\bar{B}^{0} \rightarrow D^{*+} \ell^{-} \bar{\nu}_{\ell}$, as shown in Figure 5.7.

In the $B$ rest frame, the lepton and the $D^{*+}$ should be released in approximately opposite directions by the $B$ decay, depending on the energy of the neutrino. Therefore, a cut is made on the angle between them: $\cos \theta_{D^{*}, \ell}<0$. Figure 5.8 shows the distribution of this angle for signal and for background leptons. Signal events are peaked at $\cos \theta=-1$, while background events are evenly distributed over all angles.

\subsubsection{Vertex refit}

Once the $D^{*+}$ and lepton candidates have been identified, the event is refitted with a special vertex algorithm. ${ }^{[47]}$ It uses a constrained vertex fitting technique that treats the

\footnotetext{
${ }^{\mathrm{c}}$ For each cut statement, the boolean true is retained, and the boolean false is removed.
} 


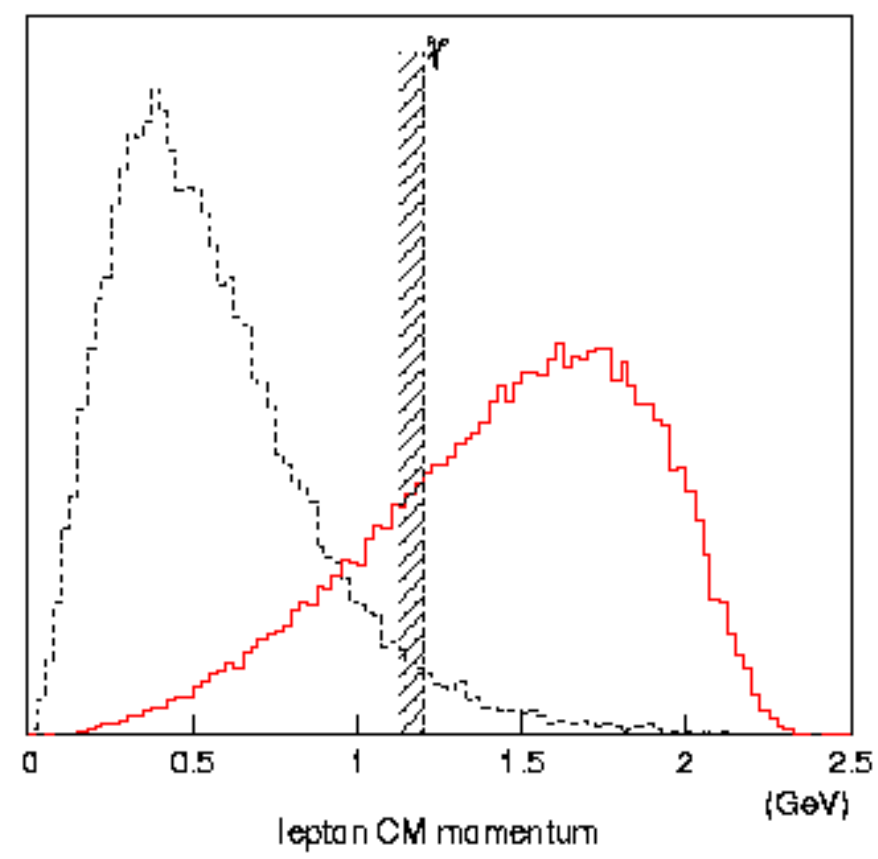

Figure 5.7: Lepton momentum spectrum in CM frame for signal (solid line) and background (dashed line) leptons from generic $B$ MC. ${ }^{[44]}$

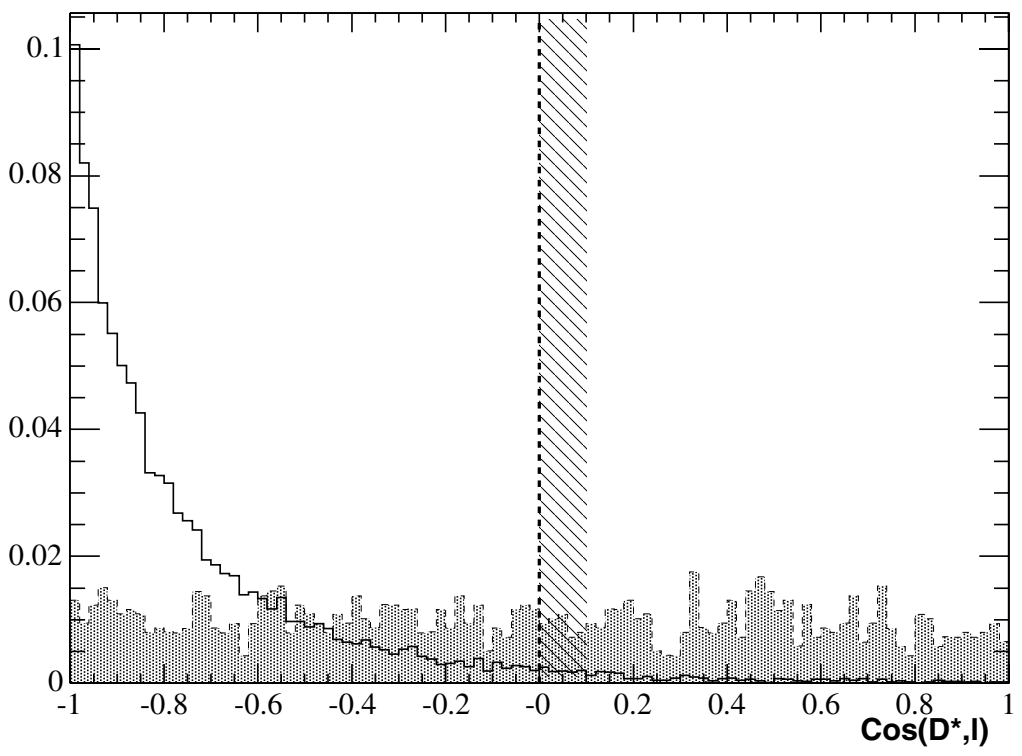

Figure 5.8: $\cos \theta_{D^{*}, \ell}$ for $\bar{B}^{0} \rightarrow D^{*+} \ell^{-} \bar{\nu}_{\ell}$ signal (open histogram) and background (filled) leptons taken from generic $B$ MC. Both histograms are normalized to unit area. In almost all of the signal events, the $D^{*+}$ and the $\ell^{-}$are in opposite hemispheres in the CM frame. 
lepton, beam-spot, $D^{*}$, and its daughters simultaneously to find the best vertex. The algorithm explicitly incorporates a flight distance for the $D^{0}$ and correctly considers the correlated errors in the fit. It provides a much improved estimate of $\delta m$ and the $D^{*+} \ell^{-}$ vertex of the $B$.

The list of inputs, unknowns and constraints used in the vertex fit are as follows:

Inputs:

- $K$ and $\pi$ ( $D^{0}$ daughters) track parameters

- slow pion track parameters

- lepton track parameters

Unknowns:

- $D^{0}$ decay vertex displacement (relative to origin) $\left(\vec{V}_{D^{0}}\right)$

- $D^{*+}$ decay vertex displacement $\left(\vec{V}_{D^{*}}\right)$

- $D^{0}$ lifetime $\left(\tau_{D^{0}}\right)$

Constraints:

- $x y$ vertex constraint at $\vec{V}_{D^{0}}$ for $D^{0}$ daughters

- $z$ vertex constraint at $\vec{V}_{D^{0}}$ for $D^{0}$ daughters

- $x y$ vertex constraint at $\vec{V}_{D^{*}}$ for $D^{0}, \pi_{\text {slow }}, \ell$

- $z$ vertex constraint at $\vec{V}_{D^{*}}$ for $D^{0}, \pi_{\text {slow }}, \ell$

- $\vec{V}_{D^{0}}-\vec{V}_{D^{*}}=c \tau_{D^{0}} / m_{D^{0}} \times \sum_{i}^{D^{0} \text { daughters }} \vec{p}_{i}$

- beam-spot is applied as a constraint on $\vec{V}_{D^{*}}$ and the lepton

The output of the algorithm is

- improved versions of the inputs

- the values of the unknowns

- all correlations.

The refitted value for $\delta m$ is used in making the cuts shown in Figure 5.5, and in the fits used in characterizing the combinatoric background. Additionally, a cut is made on the $\chi^{2}$ probability of this fit at 0.01 to eliminate events which fail the refit. 


\subsubsection{Further Cuts}

Once the $D^{*+} \ell^{-}$candidate has been identified, the thrust angle, $\theta_{\text {thrust }}$ is calculated between its thrust and that of the rest of the event in the CM frame. The thrust of a group of particles is a vector representing the weighted average momentum of the group. In collisions where high energy quarks are produced, there is often a cone of many particles emerging from the collision, known as a jet. The thrust of a jet is a good measure of the direction of the initial quark which produced the jet.

At the $\Upsilon(4 S)$ resonance, the two $B$ mesons decay nearly at rest, and no jet is produced, so the decay topology is fairly spherical, and $\theta_{\text {thrust }}$ has a flat distribution. Events coming from the continuum tend to be jetty; therefore, the angle between the two thrusts tends to be close to zero or 180 degrees. Thus, the continuum events will be peaked near $\left|\cos \theta_{\text {thrust }}\right|=1$. A cut is applied at $\left|\cos \theta_{\text {thrust }}\right|<0.85$ in order to separate signal from continuum background, as can be seen in Figure 5.9.

An attempt is also made to reconstruct the vertex of the other $B$ in the event regardless of its type of decay. The event is rejected if the vertex cannot be determined from at least two remaining tracks. This helps to further reduce the continuum background by requiring that both $B$ mesons are seen to some degree in the event.

After all the selection criteria are applied, there are rare cases in which there are more than one $D^{*+} \ell^{-}$candidate per event. Only one $D^{*+} \ell^{-}$per event is used in the final data

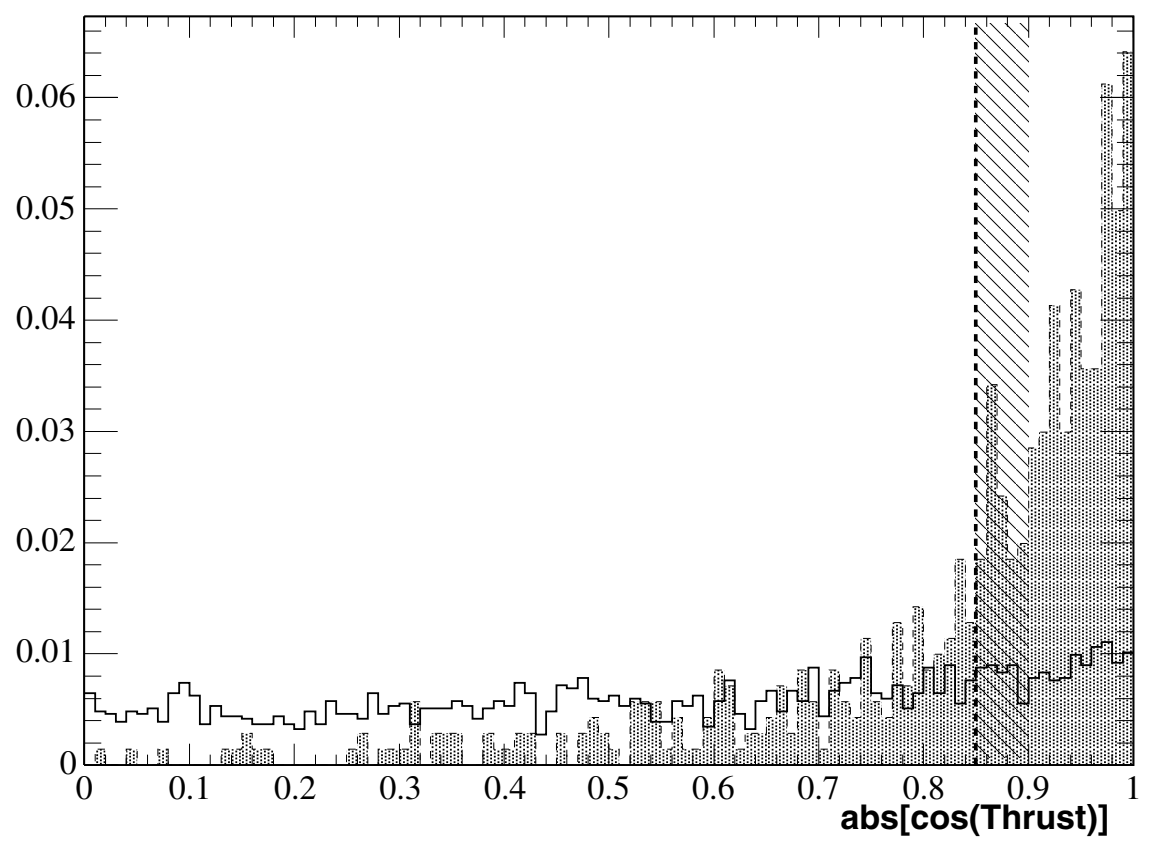

Figure 5.9: Distribution of $\cos \theta_{\text {thrust }}$ between $D^{*+} \ell^{-}$candidate and the rest of the event for signal MC events (open histogram) and continuum background (shaded histogram) from generic charm MC. 
sample. The $D^{0}$ mass is used for the final selection in these cases. The candidate with the $D^{0}$ mass closer to the nominal mass is selected. For $D^{*+} \ell^{-}$candidates which are both reconstructed from the same $D^{0}$ candidate, no criterion is used to distinguish them; the first candidate in the list is chosen for the event. This occurs only $0.5 \%$ of the time.

\subsection{Monte Carlo Corrections}

The modeling of the Monte Carlo simulated data has been studied extensively within the $B A B A R$ collaboration to ensure that is represents the data as accurately as possible. In certain cases, systematic discrepancies exist between the MC and the data. Therefore, some corrections need to be applied after the MC samples have been produced in order to improve their agreement to the data.

\subsubsection{Tracking Efficiency Correction}

Tracking efficiency studies as described in Section 4.1.2 have been performed on the MC simulated data sets. ${ }^{[48]}$ In order to correct any efficiency differences, a tracking efficiency table has been created. It breaks the data into bins of $p_{T}, \theta, \phi$, and track multiplicity, as defined by the number of GoodTracksVeryLoose in the event. This table applies to tracks passing the GoodTracksLoose selector.

For each track passing GoodTracksLoose, the event is weighted by the correction factor in the appropriate bin of the tracking efficiency table. This is applied to three of the four tracks in the event: the muon, and the $D^{0}$ daughters. The slow pion is taken from the GoodTracksVeryLoose list, so this method does not apply. For GoodTracksVeryLoose, no correction is applied.

These corrections carry inherent systematic errors. For the tracks in GoodTracksLoose, a systematic error of $0.8 \%$ per track is applied. This error is correlated for each track, so the systematic error is added linearly, giving a total error for the three tracks of $2.4 \%$. For GoodTracksVeryLoose, unless the event has a track multiplicity of less than five charged tracks, a systematic error of $1.3 \%$ needs to be applied for each track because the multiplicity dependence on the efficiency is not well known.

\subsubsection{Slow $\pi$ Efficiency Correction}

Extra caution must be taken with the efficiency of the slow pion. Because some of the tracks enter the DCH and reach the GoodTracksLoose list, the strategy of applying no correction is not ideal. Instead, the slow pion efficiency has been studied separately within the collaboration to determine the proper correction needed for the MC. ${ }^{[4]}$

The relative efficiency of the slow pion is measured as a function of helicity. The helicity angle, $\theta^{*}$ is defined as the angle between the slow pion direction of motion in the $D^{*}$ frame and the direction of motion of the $D^{*}$. For a given $D^{*}$ momentum, the relation between the energy of the slow pion and $\cos \theta^{*}$ is linear. So the relationship between 


\begin{tabular}{|c|c|c|c|}
\hline sample & DCH voltage $(\mathrm{V})$ & $\beta(\mathrm{MeV} / c)^{-1}$ & $p_{0}(\mathrm{MeV} / c)$ \\
\hline Run 1 & 1900 & $175 \pm 19$ & $66.0 \pm 0.4$ \\
Run 1 & 1960 & $147 \pm 17$ & $65.2 \pm 0.5$ \\
Run 2 & 1930 & $170 \pm 11$ & $65.2 \pm 0.2$ \\
Run 3 & 1930 & $170 \pm 13$ & $65.8 \pm 0.3$ \\
generic MC & all & $148 \pm 16$ & $65.0 \pm 0.4$ \\
\hline
\end{tabular}

Table 5.2: Parameters for slow pion efficiency function.

the momentum of the slow pion and $\cos \theta^{*}$ is roughly linear. By examining the helicity spectrum, the efficiency function has been determined:

$$
\epsilon(p)= \begin{cases}1-\left[\beta\left(p-p_{0}\right)+1\right]^{-1} & \text { if } \mathrm{p}>\mathrm{p}_{0} \\ 0 & \text { if } \mathrm{p} \leq \mathrm{p}_{0}\end{cases}
$$

where $p_{0}$ is the starting point, and $\beta$ describes how fast the efficiency function rises to a plateau. These parameters vary depending upon the sample being studied. They are shown in Table 5.2.

For each slow pion in the MC samples, the relative efficiency is calculated from Eq. 5.1 using both the MC parameters and the data parameters with the appropriate DCH voltage. The ratio of the two values is applied to the weight of the event.

This correction is only slight, especially for MC representing data acquired with the $\mathrm{DCH}$ at $1960 \mathrm{~V}$ in the latter half of Run 1. The parameters for the MC efficiency are almost identical to those for the data during that period. Calculating the relative efficiency for the $B^{0}, B^{ \pm}$, and $c \bar{c}$ generic MC averaged over momentum, using both the data and MC parameters from Table 5.2 gives:

- Average efficiency based on data parameters $=0.99770 \pm 0.00003$.

- Average MC efficiency based on MC parameters $=0.9979 \pm 0.0001$.

The ratio of the two yields an averaged correction factor of $0.9998 \pm 0.0001$. For data from Runs $2 \& 3$, where the parameters differ more significantly, the overall correction is approximately 1\%. The error in the average correction factor is negligible here. So the systematic error applied to the slow pion track is only the error from the GoodTracksVeryLoose list, or $1.3 \%$.

\subsubsection{PID Efficiency Correction}

The particle control samples described in Section 4.2.4 allow the efficiencies of the PID selectors to be studied on data. The selector efficiency is dependent on both the momentum of the particle and its position in the detector. Tables are made from the control samples with bins of $(p, \theta, \phi)$. Each bin gives the total number of particles as well as the number passing the particular PID selector. From this, the efficiency and its statistical error are stored. This is done both for the ID and mis-ID rates (i.e. The muon selector is 
applied to the pion control sample to measure the mis-ID rate of pions being accepted as muons.) PID efficiency tables have also been generated from MC simulated data. From the two tables, a correction factor is obtained based on the ratio of the efficiencies. This is applied for both the kaon and the muon in the event, since both are selected using the particle identification.

$$
C_{P I D}=\frac{\epsilon^{d a t a}\left(\mu \rightarrow \mu^{V T}\right)}{\epsilon^{M C}\left(\mu \rightarrow \mu^{V T}\right)} \times \frac{\epsilon^{d a t a}\left(K \rightarrow K^{n o t-\pi}\right)}{\epsilon^{M C}\left(K \rightarrow K^{n o t-\pi}\right)} .
$$

Each event is reweighted by this correction factor.

In order to correct for the mis-ID rates, each muon or kaon candidate is identified with MC truth. The appropriate efficiency table is then chosen based on the true particle ID and selector being used. For example, if the muon candidate is actually a pion, the efficiency table used is that for a pion being misidentified as a muon.

\subsubsection{Beam Energy Correction}

Since the $B^{0}$ cannot be completely reconstructed because of the missing neutrino, the CM momentum of the $B, p^{*}\left(B^{0}\right)$ is derived from the beam energy. However, the beam conditions periodically change, especially after periods of beam tuning and development. The result is that beam energy may be a few MeV off the $\Upsilon(4 S)$ resonance peak of $10.58 \mathrm{GeV}$. This is not accounted for in the MC modeling. Therefore, there is a small discrepancy in the $p^{*}\left(B^{0}\right)$ value between data and MC. This has been studied within the collaboration, ${ }^{[50]}$ with the following results:

- $p^{*}\left(B^{0}\right)=326.1 \pm 2.3 \mathrm{MeV} / c$

- $p_{M C}^{*}\left(B^{0}\right)=319.6 \pm 1.3 \mathrm{MeV} / c$

- $p^{*}\left(B^{+}\right)=335.8 \pm 1.0 \mathrm{MeV} / c$

- $p_{M C}^{*}\left(B^{+}\right)=325.1 \pm 0.8 \mathrm{MeV} / c$

In order to correct for this difference, the $p^{*}\left(B^{0}\right)$ for each $\mathrm{MC}$ event is multiplied by $326.1 / 319.6$, and the $B^{0}$ energy is recalculated.

\section{Summary of Event Selection}

At this point, the full Run 1 data has been reduced to a reasonably clean sample of $\bar{B}^{0} \rightarrow$ $D^{*+} \mu^{-} \bar{\nu}_{\mu}$ events. No further selection criteria are applied to the data to refine the signal events. However, some backgrounds still exist in the sample which must be understood. These backgrounds are well modeled by the MC simulated data, especially after the corrections have been applied. The $\mathrm{MC}$ truth is used to isolate the $\bar{B}^{0} \rightarrow D^{*+} \mu^{-} \bar{\nu}_{\mu}$ signal and the different backgrounds so that they can be understood and separated in the data set. 


\section{Chapter 6}

\section{Background Characterization}

The reconstruction and selection criteria described in Chapter 5 has reduced the Run 1 data and the MC down to sets of events which are all consistent with coming from a $\bar{B}^{0} \rightarrow D^{*+} \mu^{-} \bar{\nu}_{\mu}$ decay. ${ }^{\text {a }}$ The $D^{*+}$ candidates have been reconstructed from a $D^{0}$ candidate and a slow charged pion. The $\mu^{-}$has been chosen such that it is consistent with having been produced by the same decay as the $D^{*+}$. The overall event is also consistent with an $e^{+} e^{-} \rightarrow \Upsilon(4 S)$ interaction.

Unfortunately, this does not guarantee that the events in the data set are actual $\bar{B}^{0} \rightarrow D^{*+} \mu^{-} \bar{\nu}_{\mu}$ signal events. The data set consists of approximately $70 \%$ signal and $30 \%$ background events, as will be shown. The composition of the data set is

1. Events originating from $B \bar{B}$ candidates with a real $D^{*}$ :

(a) $\bar{B}^{0} \rightarrow D^{*+} \mu^{-} \bar{\nu}_{\mu}$ signal events.

(b) $D^{*+} \mu^{-} \bar{\nu}_{\mu} \pi$ background: Decays of type:

i. $\bar{B}^{0} \rightarrow D^{* *+} \mu^{-} \bar{\nu}_{\mu}$ with $D^{* *+} \rightarrow D^{*+} \pi^{0}$.

ii. $B^{-} \rightarrow D^{* * 0} \mu^{-} \bar{\nu}_{\mu}$ with $D^{* * 0} \rightarrow D^{*+} \pi^{-}$.

iii. $\bar{B}^{0} \rightarrow D^{*+} \mu^{-} \bar{\nu}_{\mu} \pi^{0}$ nonresonant.

iv. $B^{-} \rightarrow D^{*+} \mu^{-} \bar{\nu}_{\mu} \pi^{-}$nonresonant.

(c) muon backgrounds:

i. Correlated muon: Decays of type $B \rightarrow D^{*+} X$ with $X \rightarrow Y \mu^{-} \bar{\nu}_{\mu}$.

ii. Uncorrelated muon: $D^{*}$ and $\mu$ come from different $B$ 's in the event.

iii. Fake muon: From hadrons passing the muon very-tight selector.

2. Continuum background: Events of type $c \bar{c} \rightarrow D^{*+} \mu^{-} \bar{\nu}_{\mu} X$.

3. Combinatoric background: $D^{*}$ candidate is not properly reconstructed.

In order to calculate the $\bar{B}^{0} \rightarrow D^{*+} \mu^{-} \bar{\nu}_{\mu}$ branching fraction, an accurate count of the $D^{*+} \mu^{-} \bar{\nu}_{\mu}$ signal events must be obtained. This also requires an accurate count of the different background events, so that all events are accounted for. The signal and each of these backgrounds and the means of characterizing them are explained in this chapter. The main resource for isolating pure samples of signal and background is the MC truth information.

a Since only the muon mode is of interest, the electron mode is not used. However, the backgrounds described here also apply to the electron mode. The only modification to the selection criteria to choose the electron mode is to change to PID selector described in Section 5.4.3. 


\subsection{Monte Carlo Truth}

\subsubsection{Truth Association}

Each simulated event carries a complete list of the particles produced in the event, with the full information generated for each particle. This includes both the final state particles, and the short lived particles which decay in flight, often before leaving any signal in the detector. Unfortunately, when the event is processed to generate the raw detector signals in order to mimic the real data, some of this information is not retained. The truth list remains part of each event, but the detector hits do not contain this information.

In order to use the truth information, each charged track or neutral signal must be associated back to a particle in the truth list. This is done during OPR. After the tracks have been reconstructed using the detector hits, the truth association software compares the momentum vector $(p, \theta, \phi)$ of each measured track with the tracks in the truth list and calculates a $\chi^{2}$ value

$$
\chi^{2}=\frac{\left(p_{\text {meas }}-p_{\text {truth }}\right)^{2}}{\sigma_{p_{\text {meas }}}^{2}}+\frac{\left(\theta_{\text {meas }}-\theta_{\text {truth }}\right)^{2}}{\sigma_{\theta_{\text {meas }}}^{2}}+\frac{\left(\phi_{\text {meas }}-\phi_{\text {truth }}\right)^{2}}{\sigma_{\phi_{\text {meas }}}^{2}}
$$

Each measured track is associated to the truth track which produced the lowest $\chi^{2}$ value.

There is a small probability $(\sim 1 \%)$ that the truth associator will mistakenly match the wrong tracks, especially for low momentum tracks such as the slow pion. This effect is expected to be small enough that it doesn't matter in this analysis, but it will be examined with the other systematic studies described in Chapter 8.

\subsubsection{Truth Variables}

Monte Carlo truth information is used to isolate the different background samples from the simulated data sets in this analysis. Several variables are created to characterize the $D^{*}$ and muon candidates, and their parents. Their definitions are ${ }^{[51]}$ 
real $D^{0}$

real $D^{*}$

$D^{*}$ from $B$

$\mu$ from $B$

$\mu$ from same $B$

direct $\mu$

$\mu I D$

$B_{\mu} I D$

$D^{*}$ parent ID
This variable is designed to accommodate several different $D^{0}$ decay modes. It checks to see that all the $D^{0}$ 's daughters are from the same $D^{0}$ and the number of "final" state particles $\left(K^{ \pm}, K_{S}^{0}, \pi^{ \pm}, \pi^{0}\right)$ are the same, and that the daughter IDs match. It does not explicitly check the number of $D^{0}$ daughters because the $D^{0}$ may decay to a resonance first and the number of daughters could be different from the number of composite daughters.

This checks to see that the $D^{0}$ daughter is real and that the soft pion daughter and the $D^{0}$ come from the same $D^{*}$.

This checks to see that the $D^{*}$ is real, and it comes from a $B^{0}$ or $B^{ \pm}$. It can be either from a direct decay or from a cascade.

The muon candidate is from a $B^{0}$ or $B^{ \pm}$. The true particle ID isn't considered; a separate variable is used for that. The muon also can be from either a direct decay or a cascade.

The $D^{*}$ must be real. The muon and the $D^{*}$ are from the same $B^{0}$ or $B^{ \pm}$.

The muon is directly from a $B$, not from cascade.

The MC particle ID of the muon candidate.

The MC particle ID of the B parent of the muon candidate. This helps in distinguishing $B^{0}$ from $B^{ \pm}$.

The MC particle ID of the $D^{*}$ parent.

\section{2 $\cos \theta_{B, D^{*} \ell}$ Angular Variable}

An important variable for discriminating between $\bar{B}^{0} \rightarrow D^{*+} \mu^{-} \bar{\nu}_{\mu}$ signal events and $B \rightarrow D^{*+} \mu^{-} \bar{\nu}_{\mu} \pi$ or other background is the angle between the $B$ meson and the $D^{*+} \ell^{-}$ candidate in the $\mathrm{CM}$ frame. This value can be calculated from simple 4-vector algebra with the assumption that the only missing particle is the zero mass neutrino.

$$
\begin{aligned}
p_{B}^{\mu}= & p_{D^{*}}^{\mu}+p_{\mu}^{\mu}+p_{\nu}^{\mu} \\
\left(p_{\nu}^{\mu}\right)^{2}= & \left(p_{B}^{\mu}-p_{D^{*+\ell^{-}}}^{\mu}\right)^{2} \\
m_{\nu}^{2}=0= & m_{B}^{2}+m_{D^{*+\ell^{-}}}^{2}+2\left(E_{B} E_{D^{*+\ell^{-}}}-\left|\vec{p}_{B}\right|\left|\vec{p}_{D^{*+\ell^{-}}}\right| \cos \theta_{B, D^{*} \ell}\right) \\
& \cos \theta_{B, D^{*} \ell}=-\frac{m_{B}^{2}+m_{D^{*+\ell^{-}}}^{2}-2 E_{B} E_{D^{*+} \ell^{-}}}{2\left|\vec{p}_{B}\right|\left|\vec{p}_{D^{*+} \ell^{-}}\right|}
\end{aligned}
$$

Since $\cos \theta_{B, D^{*} \ell}$ comes from a physical angle, the value should fall between $[-1,1]$ for real $\bar{B}^{0} \rightarrow D^{*+} \mu^{-} \bar{\nu}_{\mu}$ events. In the case of the $D^{*+} \mu^{-} \bar{\nu}_{\mu} \pi$ background, there is an extra pion, so the missing mass is not zero for this background. Therefore, the calculation of $\cos \theta_{B, D^{*} \ell}$ will be missing a component, causing it to move toward more negative values and into the nonphysical region, $\cos \theta_{B, D^{*} \ell}<-1$. 
The other types of backgrounds also show different distributions of this variable, including extensions into the range of $\cos \theta_{B, D^{*} \ell}>1$. This distribution will be used to determine the numbers of signal and background events in the data. If the MC simulation correctly models the data, then it will have the same number of events as the data for the signal and each type of background, and the combined shape of the samples will match the shape of the data set. Therefore, the areas of the signal and background samples will be fitted to the data with the total number of events constrained to the data. This fitting method to count the signal and background events and determine the branching fraction will be described in Chapter 7 . The $\cos \theta_{B, D^{*} \ell}$ plot will be shown for each signal or background mode isolated from MC truth.

\section{3 $\quad \bar{B}^{0} \rightarrow D^{*+} \mu^{-} \bar{\nu}_{\mu}$ Signal}

The $\bar{B}^{0} \rightarrow D^{*+} \mu^{-} \bar{\nu}_{\mu}$ events are modeled both in generic $B^{0} \mathrm{MC}$, and in single-mode $\bar{B}^{0} \rightarrow D^{*+} \ell^{-} \bar{\nu}_{\ell}$ MC. The truth requirements to be labeled a signal event are the same for both generic and single-mode MC. There must be a real $D^{*}$ directly from $B$ and a $\mu$ directly from same $B$. The $\mu I D$ must be that of a muon, and the $D^{*}$ parent ID must be a $B^{0}$. There must also be no extra daughter pions from the $B^{0}$. Once the signal events are isolated in the generic and single-mode MC samples, the two are merged. The total number of signal events in the merged sample is 13,050 .

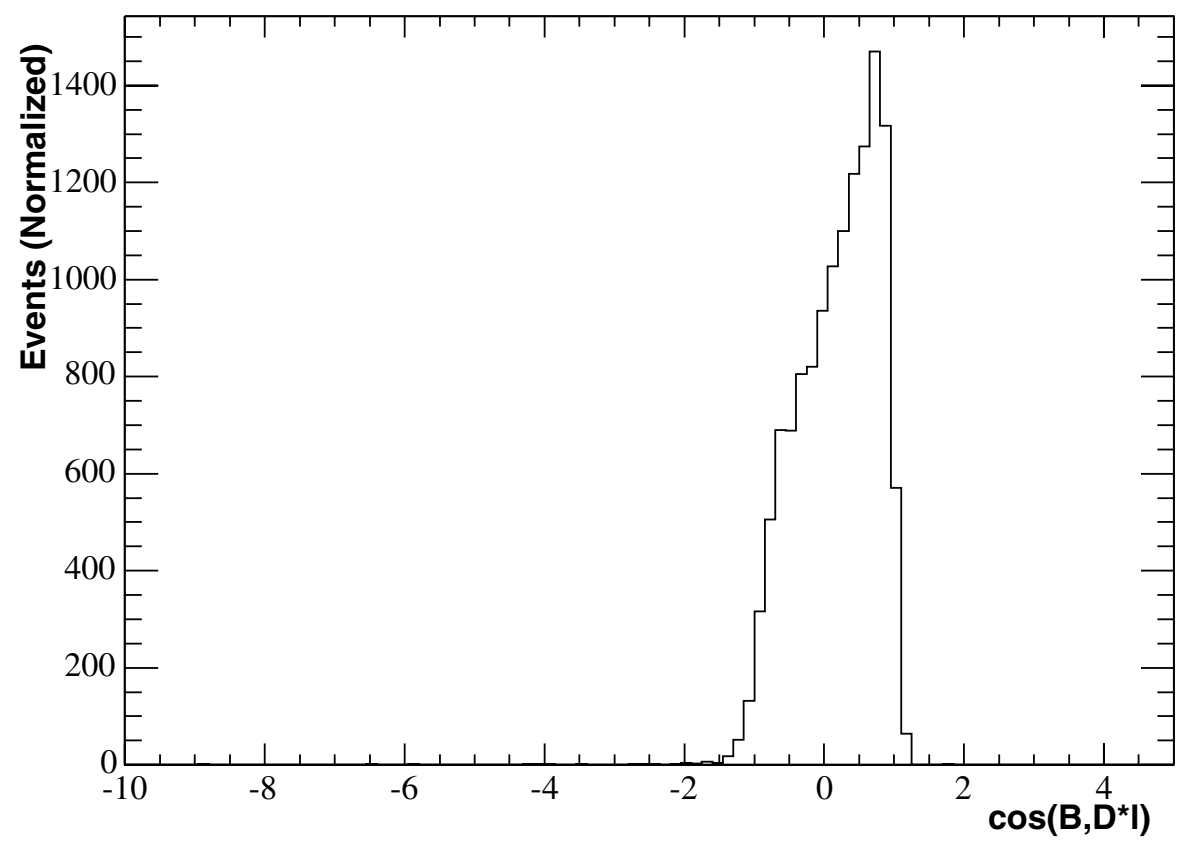

Figure 6.1: $\cos \theta_{B, D^{*} \ell}$ for $\bar{B}^{0} \rightarrow D^{*+} \mu^{-} \bar{\nu}_{\mu}$ signal events from generic $B^{0}$ and $\bar{B}^{0} \rightarrow$ $D^{*+} \mu^{-} \bar{\nu}_{\mu}$ single-mode MC truth. 
The $\cos \theta_{B, D^{*} \ell}$ distribution for $\bar{B}^{0} \rightarrow D^{*+} \mu^{-} \bar{\nu}_{\mu}$ signal events is shown in Figure 6.1. The signal events fall almost entirely within the range $[-1,1]$. There is some spread due to tracking resolution effects, and a small negative tail due to final state radiation. As will be seen, this distribution is very different for the background modes.

\section{$6.4 B \rightarrow D^{*+} \mu^{-} \bar{\nu}_{\mu} \pi$ Background}

This background corresponds to decays of $B \rightarrow D^{*+} \mu^{-} \bar{\nu}_{\mu}$ with an additional pion. This can happen either directly, or resonantly through a higher $D$ meson excited state which are collectively referred to as $D^{* *}$ mesons. Because these events produce a $D^{*}$ and a muon from the same $B$ parent, and because no attempt is made to look for the additional pion, they generally pass the selection criteria and present a significant background. Current measurements suggest that the sum of all (resonant and nonresonant) decays of $B \rightarrow$ $D^{*+} \mu^{-} \bar{\nu}_{\mu} \pi$ account for about $20 \%$ of the total semileptonic width. ${ }^{[52]}$ The masses and branching fractions for the $D^{* *}$ and nonresonant modes considered are shown in Table 6.1 .

There are four $D^{* *}$ states in the generic MC: $D_{1}, D_{0}^{*}, D_{1}^{*}$, and $D_{2}^{*}{ }^{\text {. }}$ Each can be either charged or neutral with isospin symmetry. All are orbitally excited states with orbital angular momentum $l=1$. The total angular momentum of the meson, $J$, is indicated by the subscript: $D_{J}$. The $D_{1}$ is a singlet ${ }^{1} P_{1}$ state, while the $D_{0}^{*}, D_{1}^{*}$, and $D_{2}^{*}$ are three triplet ${ }^{3} P_{0},{ }^{3} P_{1}$, and ${ }^{3} P_{2}$ states.

In HQET, the $D$ (and $B$ ) meson is analogous to the hydrogen atom, with the light quark orbiting the heavy quark nucleus. Therefore, they can be classified not only by $J$, but also the light quark angular momentum:

$$
\vec{j}=\vec{l}+\vec{s},
$$

where $s$ is the spin of the light quark and $l$ is the orbital angular momentum, as shown in Figure 6.2. The total angular momentum is given by

\footnotetext{
${ }^{\mathrm{b}}$ The $D_{1}^{*}$ is also referred to as the $D_{1}^{\prime}$ in the literature.
}

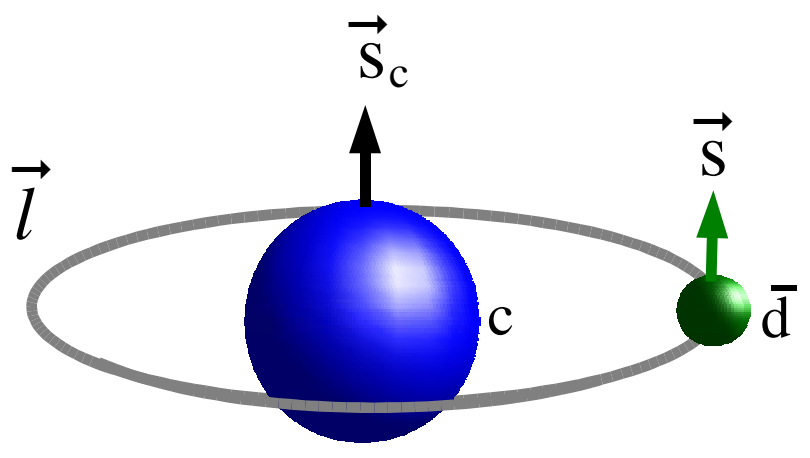

Figure 6.2: Pictorial view of the $D$ meson. 


\begin{tabular}{|l|c|c|c||c|c|c|}
\hline$B$ & $\begin{array}{c}D^{* *} \text { mass } \\
\left(\mathrm{GeV} / c^{2}\right)\end{array}$ & $\begin{array}{c}D^{* *} \\
\text { Spin }\end{array}$ & $\begin{array}{c}\mathcal{B} \\
(\%)\end{array}$ & $\begin{array}{l}D^{* *} \\
\text { decay mode }\end{array}$ & $\begin{array}{c}\mathcal{B} \\
(\%)\end{array}$ & $\begin{array}{c}\text { Overall } \mathcal{B} \\
\left(\times 10^{-4}\right)\end{array}$ \\
\hline $\bar{B}^{0} \rightarrow D_{1}^{+} \mu^{-} \bar{\nu}_{\mu}$ & 2.427 & $\uparrow \downarrow$ & 0.56 & $D_{1}^{+} \rightarrow D^{*+} \pi^{0}$ & 33 & 18.5 \\
$\bar{B}^{0} \rightarrow D_{0}^{*+} \mu^{-} \bar{\nu}_{\mu}$ & 2.40 & $\uparrow \uparrow$ & 0.20 & $D_{0}^{*+} \rightarrow D^{*+} \pi^{0}$ & 0 & 0 \\
$\bar{B}^{0} \rightarrow D_{1}^{*+} \mu^{-} \bar{\nu}_{\mu}$ & 2.461 & $\uparrow \uparrow$ & 0.37 & $D_{1}^{*+} \rightarrow D^{*+} \pi^{0}$ & 33 & 12.2 \\
$\bar{B}^{0} \rightarrow D_{2}^{*+} \mu^{-} \bar{\nu}_{\mu}$ & 2.459 & $\uparrow \uparrow$ & 0.37 & $D_{2}^{*+} \rightarrow D^{*+} \pi^{0}$ & 10.3 & 3.8 \\
$\bar{B}^{0} \rightarrow D^{*+} \mu^{-} \bar{\nu}_{\mu} \pi^{0}$ & - & - & 0.10 & - & - & 10 \\
\hline$B^{-} \rightarrow D_{1}^{0} \mu^{-} \bar{\nu}_{\mu}$ & 2.422 & $\uparrow \downarrow$ & 0.56 & $D_{1}^{+} \rightarrow D^{*+} \pi^{-}$ & 67 & 37.5 \\
$B^{-} \rightarrow D_{0}^{* 0} \mu^{-} \bar{\nu}_{\mu}$ & 2.40 & $\uparrow \uparrow$ & 0.20 & $D_{0}^{*+} \rightarrow D^{*+} \pi^{-}$ & 0 & 0 \\
$B^{-} \rightarrow D_{1}^{* 0} \mu^{-} \bar{\nu}_{\mu}$ & 2.461 & $\uparrow \uparrow$ & 0.37 & $D_{1}^{*+} \rightarrow D^{*+} \pi^{-}$ & 67 & 12.2 \\
$B^{-} \rightarrow D_{2}^{* 0} \mu^{-} \bar{\nu}_{\mu}$ & 2.4589 & $\uparrow \uparrow$ & 0.37 & $D_{2}^{*+} \rightarrow D^{*+} \pi^{-}$ & 20.9 & 7.73 \\
$B^{-} \rightarrow D^{*+} \mu^{-} \bar{\nu}_{\mu} \pi^{-}$ & - & - & 0.20 & - & - & 20 \\
\hline
\end{tabular}

Table 6.1: $B \rightarrow D^{*+} \ell^{-} \bar{\nu}_{\ell} \pi$ decay modes in generic MC. Branching fractions are shown both for the decay to the $D^{* *}$ resonance and for the production of $D^{*+} \ell^{-} \bar{\nu}_{\ell} \pi$ through that resonance. Branching fractions and masses shown are taken from the BABAR MC definition files, and are based mainly on theoretical estimates. For the non-resonant $B \rightarrow D^{*+} \ell^{-} \bar{\nu}_{\ell} \pi$ modes, the model by Goity and Roberts ${ }^{[53]}$ is used, while the ISGW2 $2^{[54]}$ model is used for the various $D^{* *}$ decay modes.

$$
\vec{J}=\vec{j}+\overrightarrow{s_{c}}
$$

where $s_{c}$ is the spin of the charm quark.

The orbitally excited $D^{* *}$ states can be organized into two doublets based on $j$, The $D_{1}$ and $D_{2}^{*}$ are in a $j=\frac{3}{2}$ doublet, while the $D_{0}^{*}$ and $D_{1}^{*}$ form a $j=\frac{1}{2}$ doublet. The $j=\frac{3}{2}$ states experience a $D$ wave decay, and therefore are narrow states. These two states have been measured and show the predicted quantum numbers and narrow widths. ${ }^{[6]}$ The $j=\frac{1}{2}$ states can only undergo $\mathrm{S}$ wave decays, and are therefore very wide states with widths on the order of hundreds of $\mathrm{MeV} / \mathrm{c}^{2}$. Because of this width, these modes are not easily distinguishable from background, and this doublet has not been observed yet.

The branching fractions of the $D^{* *}$ states are not well measured, but can be predicted theoretically. The dominant decay mode is into a $D$ or $D^{*}$ through the release of a single pion. From angular momentum conservation, the $D_{0}^{*}$ can only decay into $D \pi$. Therefore, it does not contribute to the $D^{*+} \ell^{-} \bar{\nu}_{\ell} \pi$ background, but is included for completeness. Similarly, the $D_{1}$ and $D_{1}^{*}$ can only decay into $D^{*} \pi$. The branching fractions specified in the MC definitions are based only on isospin considerations:

$$
\frac{\mathcal{B}\left(D^{* *+} \rightarrow D^{*+} \pi^{0}\right)}{\mathcal{B}\left(D^{* *+} \rightarrow D^{* 0} \pi^{+}\right)}=\frac{1}{2}
$$

Because of this isospin factor, more of this background comes from the $B^{ \pm}$than the $B^{0}$. Both decays of $D_{2}^{*} \rightarrow D^{*} \pi$ and $D_{2}^{*} \rightarrow D \pi$ are allowed by angular momentum conservation and have been observed by CLEO. ${ }^{[55,56]}$ The branching fractions in the MC are based 
on these measurements. There is little information about the branching fractions for the nonresonant decays of $\bar{B}^{0} \rightarrow D^{*+} \mu^{-} \bar{\nu}_{\mu} \pi^{0}$ and $B^{-} \rightarrow D^{*+} \ell^{-} \bar{\nu}_{\ell} \pi^{-}$, so the branching fractions in the MC are based on a theoretical model. ${ }^{[53]}$

There are also two radially excited $2 S$ states which are predicted: the ${ }^{1} S_{1}$ state $D^{\prime}$, and the ${ }^{3} S_{1}$ state $D^{*^{\prime}}$. These are also referred to in the literature as the $D(2 S)$ and $D^{*}(2 S)$. Some evidence for the $D^{*^{\prime}}$ state has been reported by the DELPHI collaboration, ${ }^{[57]}$ but the quantum numbers for the state have not been confirmed. These two states have not been included in the generic MC.

In order to characterize this background, MC samples of the different modes are extracted from generic MC using the truth information for the event. Each $D^{* *}$ state can be separately identified. However, the entire set $\left(D^{* *}\right.$ and nonresonant) is treated as a whole in this analysis. The relative contributions of the different modes is poorly known, so the composition of the background sample is only approximate. Systematic error measurements based on the relative contributions of the $D^{* *}$ modes are shown in Section 8.3.1.

This background is identified in MC truth by requiring a real $D^{*}$ from $B$ with a muon directly from the same $B$. If the $I D$ of the $D^{*}$ parent is not a $B^{0}$ or $B^{ \pm}$, then it is a $D^{* *}$. The ID of the parent is use to separate the different $D^{* *}$ modes.

If the parents of both the muon and the $D^{*}$ are the same $B^{0}$ or $B^{ \pm}$, and the parent $B$ also has a daughter pion, it is a nonresonant $D^{*+} \mu^{-} \bar{\nu}_{\mu} \pi$ event. Since the simulation of the leptonic mode includes radiative corrections, there could be extra daughters which are photons. These are acceptable in a true signal event, and are not included in the nonresonant $D^{*+} \ell^{-} \bar{\nu}_{\ell} \pi$ category. Extra daughter particles other than photons or pions would also be counted as nonresonant background, but no other particles are modeled as extra daughters in the $\bar{B}^{0} \rightarrow D^{*+} \mu^{-} \bar{\nu}_{\mu}$ decay simulation.

The total number of events for the $D^{*+} \mu^{-} \bar{\nu}_{\mu} \pi$ background, isolated from MC truth, is 584. This is simply the number of events in the generic MC sample, scaled for the relative luminosity of the MC compared to data. It is should be approximately the number of $D^{*+} \mu^{-} \bar{\nu}_{\mu} \pi$ background events in the data if the BABAR MC is well modeled, which will be determined by fitting the $\cos \theta_{B, D^{*} \ell}$ distributions.

Figure 6.3 shows the $\cos \theta_{B, D^{*} \ell}$ distribution for the $D^{*+} \mu^{-} \bar{\nu}_{\mu} \pi$ background. There is a large smearing of the distribution to well below $\cos \theta_{B, D^{*} \ell}=-1$. Thus, the effect of the extra missing mass from the extra pion is quite apparent.

\subsection{Muon Backgrounds}

The muon backgrounds considered contain a real $D^{*}$ from a $B^{0}$ or $B^{ \pm}$, but either the muon does not come from the same decay, or it is a hadron misidentified as a muon. These backgrounds are characterized by their different $\cos \theta_{B, D^{*} \ell}$ distributions. The number of events in the muon backgrounds is also determined by fitting the $\cos \theta_{B, D^{*} \ell}$ histograms. 


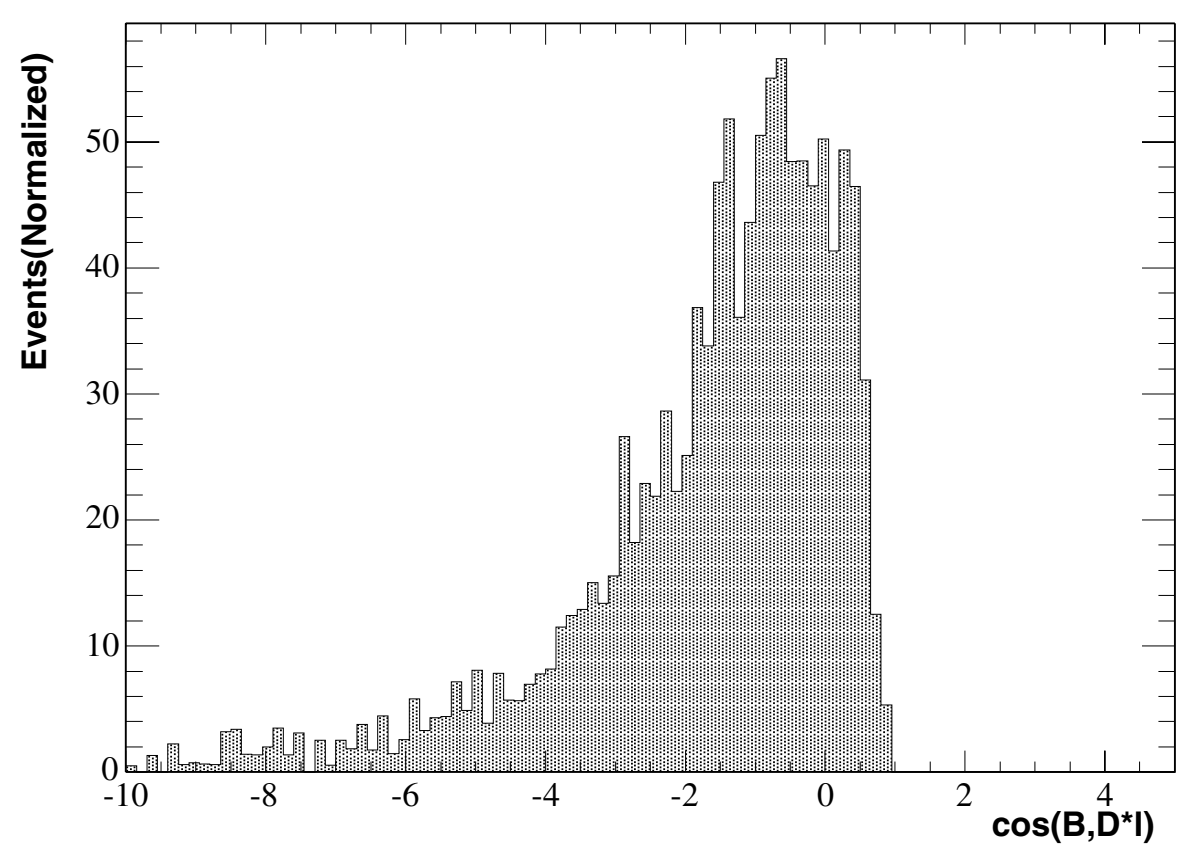

Figure 6.3: $\cos \theta_{B, D^{*} \ell}$ for $B \rightarrow D^{*+} \mu^{-} \bar{\nu}_{\mu} \pi$ background taken from generic $B^{0}$ and $B^{ \pm}$ MC using truth information.

\subsubsection{Correlated muons}

Correlated muon background is similar to the $D^{* *}$ background in that it contains a $D^{*}$ and a muon from the same $B$ meson. However, in this case, the muon does not come directly from the $B$ decay. It comes from decays of type $B \rightarrow D^{*+} X$ with $X \rightarrow Y \mu^{-} \bar{\nu}_{\mu}$ or

\begin{tabular}{|c|c|}
\hline Decay & Branching fraction \\
\hline$D^{*} D^{*} K^{*}$ & $(7.4 \%)^{\diamond}$ \\
$D_{s}^{*} D^{*}$ & $(2.0 \pm 0.7) \%$ \\
$D^{*} D$ & $\left(2.09 \times 10^{-3}\right)^{\diamond}$ \\
$D_{s} D^{*}$ & $(9.6 \pm 3.4) \times 10^{-3}$ \\
$D^{*} D^{*}$ & $\left(6.2_{-3.1}^{+4.1}\right) \times 10^{-4}$ \\
$D^{*} \tau \nu$ & $(1.6 \%)^{\diamond}$ \\
$D^{*} \rho^{ \pm}$ & $(6.8 \pm 3.4) \times 10^{-3}$ \\
$D^{*} \pi^{ \pm}\left(\pi^{0}, \eta\right)$ & $(1.5 \pm 0.5) \%$ \\
$D^{*} a_{1}^{ \pm}$ & $(1.3 \pm 0.27) \%$ \\
\hline
\end{tabular}

Table 6.2: $B$ meson decay modes contributing to the correlated background. The branching fractions listed are those used in the BABAR MC. Most values are taken from the PDG 2000 listings. ${ }^{[46]}$ The entries marked with a $\diamond$ come from theoretical estimates. 


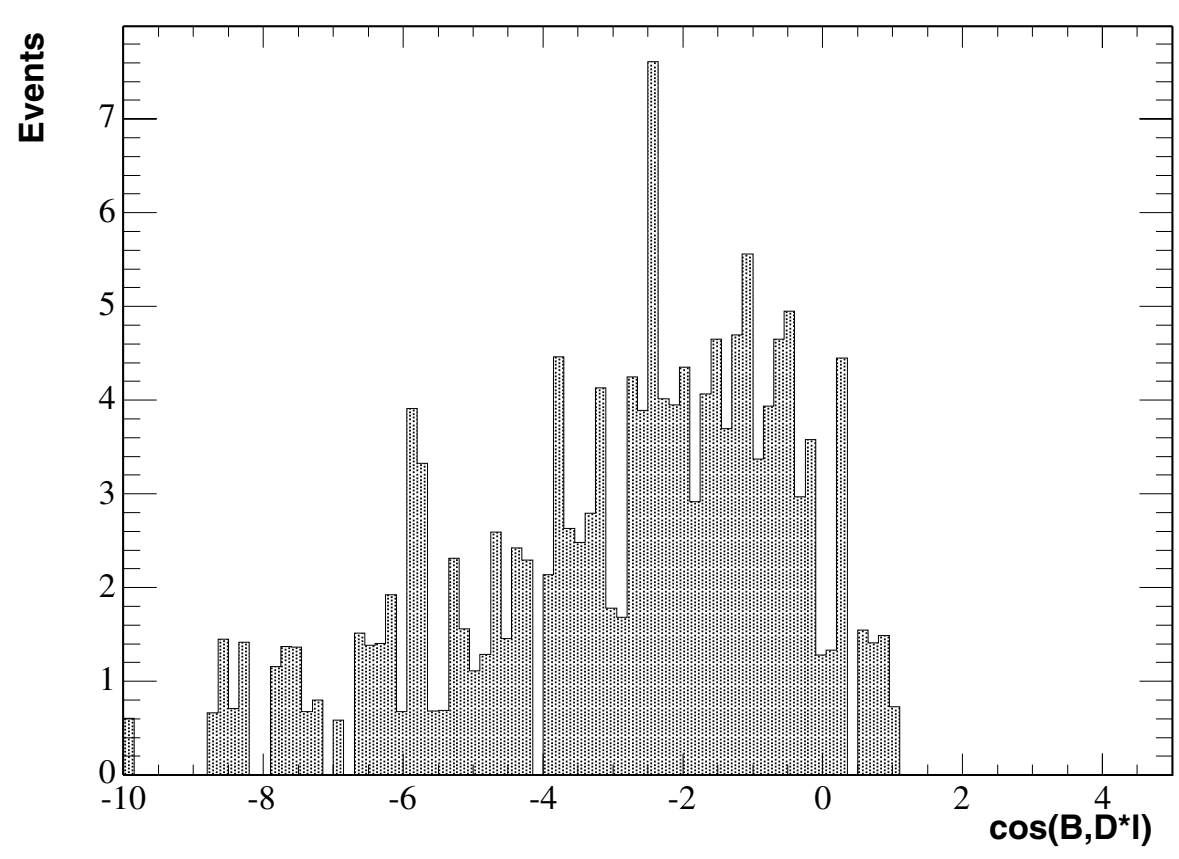

Figure 6.4: $\cos \theta_{B, D^{*} \ell}$ for correlated muon background taken from generic $B^{0}$ and $B^{ \pm} \mathrm{MC}$ truth.

$X \rightarrow Y \mu^{+} \mu^{-}$. The majority of these events are $B$ decays to a $D^{*}$ and another charmed meson, which decays semileptonically. Approximately $25 \%$ of this background comes from decays of $\bar{B}^{0} \rightarrow D^{*+} \tau^{-} \bar{\nu}_{\tau}$ with $\tau^{-} \rightarrow \mu^{-} \bar{\nu}_{\mu} \nu_{\tau}$. Another potential source is from decays of the $B$ meson to the $D^{*}$ and one of several light mesons which decay leptonically (eg. $\eta \rightarrow \gamma \mu^{+} \mu^{-}$). Table 6.2 lists the various decay modes which can contribute to the correlated muon background. Not all of these decays are well understood, so theoretical models have been used for estimates of the branching fractions of those decays in the MC simulation.

In events with a correlated background muon, the muon is produced by the intermediate decay, and its momentum tends to be lower than for $D^{*+} \mu^{-} \bar{\nu}_{\mu}$ events. Therefore, the cut $p_{\mu}^{*}>1.2 \mathrm{GeV} / c$ is very effective at reducing the correlated muon background. This can be seen in Figure 5.7 in the previous chapter, where the background shown is correlated muon background.

The shape of the $\cos \theta_{B, D^{*} \ell}$ distribution for this background is very similar to that of the $D^{*+} \mu^{-} \bar{\nu}_{\mu} \pi$ background because there is missing mass in this case, too. This is shown in Figure 6.4.

This background is isolated using MC truth by requiring a real $D^{*}$ from a $B^{0}$ or $B^{ \pm}$, along with a muon from the same $B$ with the $\mu I D$ consistent with a muon, but not a direct muon. The number of correlated muon events isolated from MC is 76 . 


\subsubsection{Uncorrelated muons}

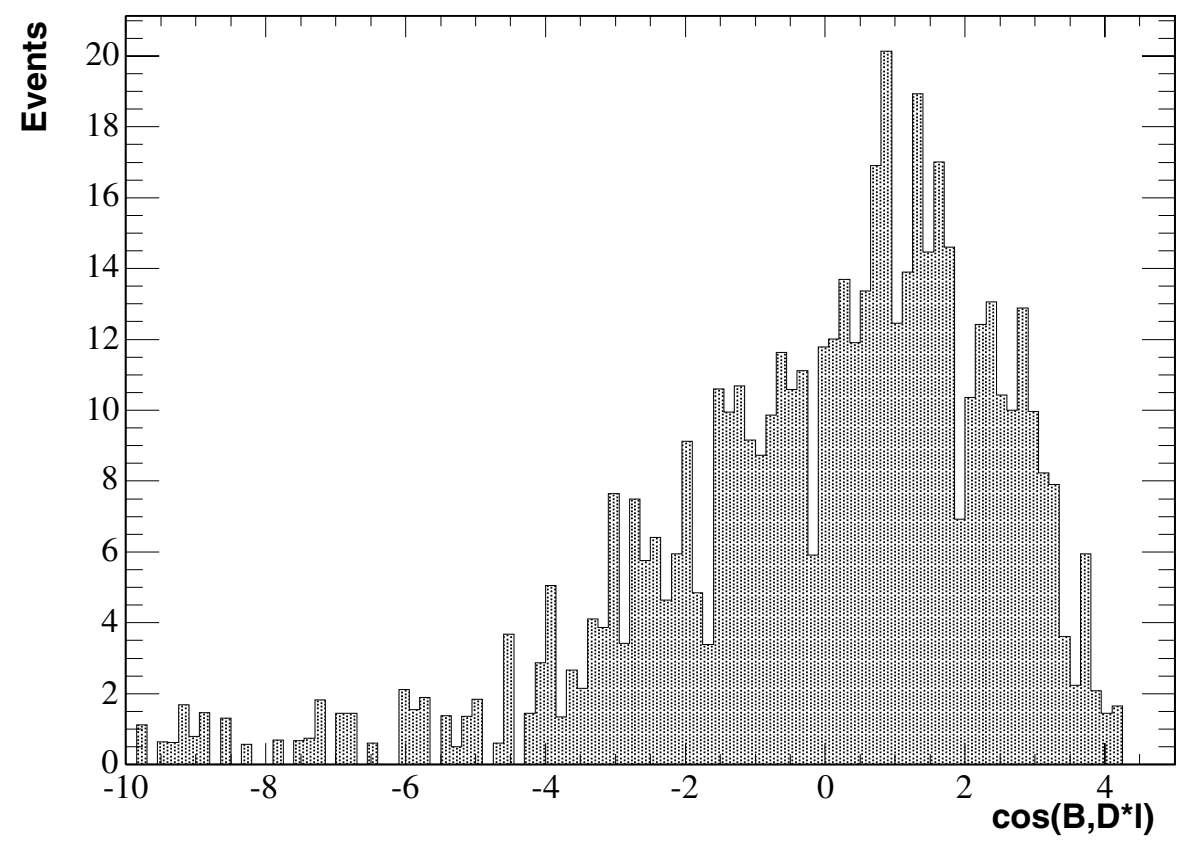

Figure 6.5: $\cos \theta_{B, D^{*} \ell}$ for uncorrelated muon background taken from generic $B^{0}$ and $B^{ \pm}$ MC truth.

The uncorrelated muon background comes from events which have both a muon and a $D^{*+}$, but they come from different $B$ mesons in the event. It is identified with a real $D^{*}$ from a $B^{0}$ or $B^{ \pm}$, a $\mu$ from a $B$, the $\mu I D$ consistent with a muon, but the muon fails the $\mu$ from same $B$. This yields 261 events from the generic $B^{0}$ and $B^{ \pm} \mathrm{MC}$ samples. Because the muon is only randomly associated with the $D^{*}$, the angular properties of this background are quite different from those of the signal events. Figure 6.5 shows that the $\cos \theta_{B, D^{*} \ell}$ distribution is significantly wider, and extends well above $\cos \theta_{B, D^{*} \ell}=1.0$, as well as below $\cos \theta_{B, D^{*} \ell}=-1.0$.

\subsubsection{Fake muons}

Because of the misidentification rates for hadrons with the Very-Tight muon selector $-1.9 \%$ for pions, $0.95 \%$ for kaons, and $0.67 \%$ for protons based on generic $B$ MC - a fraction of the $D^{*} \mu$ candidates are actually $D^{*} h$. The $\cos \theta_{B, D^{*} \ell}$ distribution for events with a misidentified muon extends into the non-physical region both above 1.0 and below -1.0 , as can be seen in Figure 6.6. It is peaked in the physical region since some of the fake muon events could be real $B \rightarrow D^{*} h$ decays.

The MC truth requirement is a real $D^{*}$ from a $B^{0}$ or $B^{ \pm}$, a $\mu$ from same $B$ and a $\mu$ $I D$ which is not an electron or muon. This produces a sample of 221 fake muon events from the generic $B$ MC. 


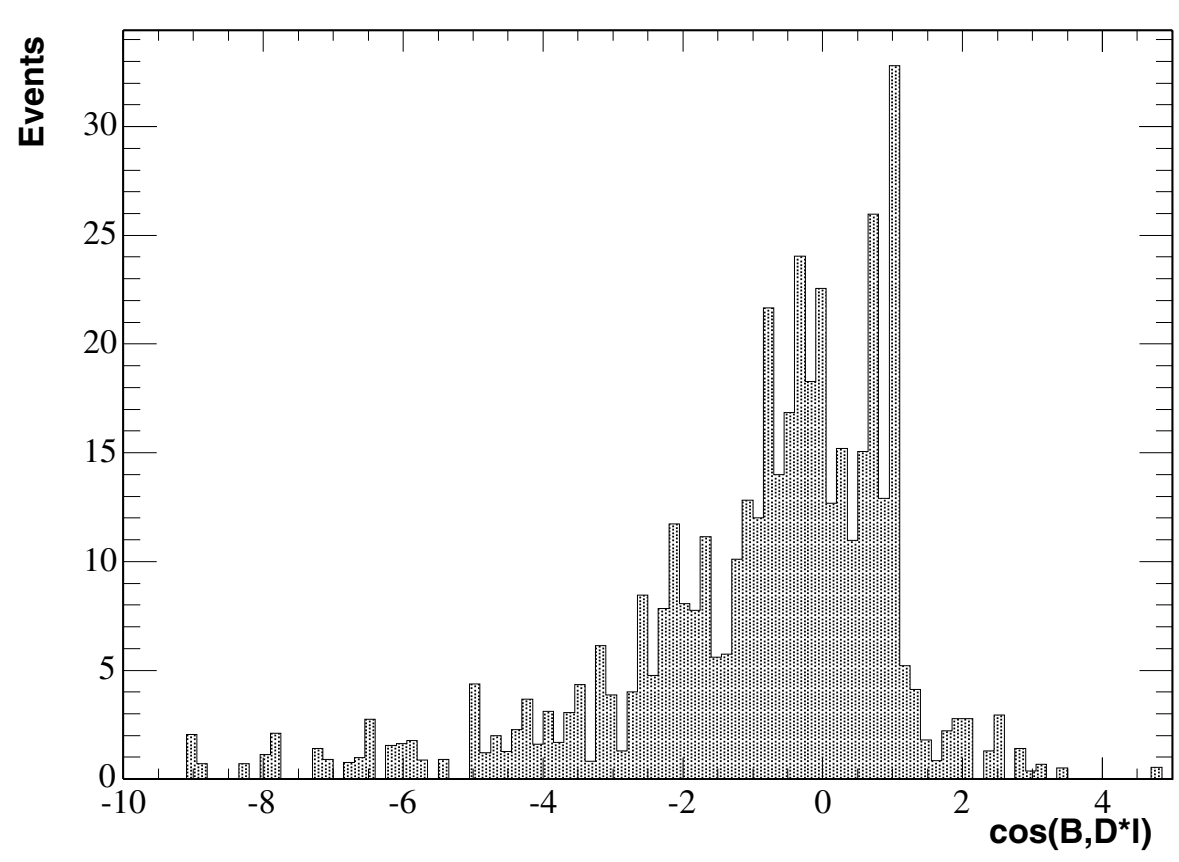

Figure 6.6: $\cos \theta_{B, D^{*} \ell}$ for fake muon background taken from generic $B^{0}$ and $B^{ \pm} \mathrm{MC}$ truth.

\subsection{Continuum Background}

As described in Section 5.3, continuum background comes from events of type $e^{+} e^{-} \rightarrow$ $(c \bar{c}, s \bar{s}, u \bar{u}, d \bar{d})$. In this analysis, only the $c \bar{c}$ events produce any significant contribution to the continuum background, which requires a real $D^{*}$ that does not come from a $B$ decay. Given that there are no $B$ mesons in the event, the calculation of $\cos \theta_{B, D^{*} \ell}$ is not based on a physical momentum for the $B$. Therefore, the $\cos \theta_{B, D^{*} \ell}$ distribution also extends well above and below the physical limits of \pm 1 , as shown in Figure 6.7. The MC truth requirement is that there must be a real $D^{*}$ which fails the $D^{*}$ from $B$ variable.

Because the source of the continuum background can be isolated in the $c \bar{c} \mathrm{MC}$, the number of background events in the data set can be easily determined. It is found by counting the number of events passing all the cuts in the $c \bar{c} \mathrm{MC}$ sample, and scaling that number by the effective luminosity of the sample. There are 281 events passing, out of 41.3 million $c \bar{c}$ events. This corresponds to a luminosity corrected value of $183 \pm 11$ continuum background events.

\subsection{Combinatoric Background}

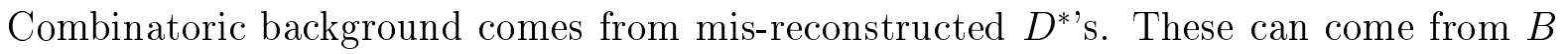
meson or continuum events, and the identity of the muon candidate is not considered. The only requirement for the combinatoric sample from MC truth is that the $D^{*}$ candidate 


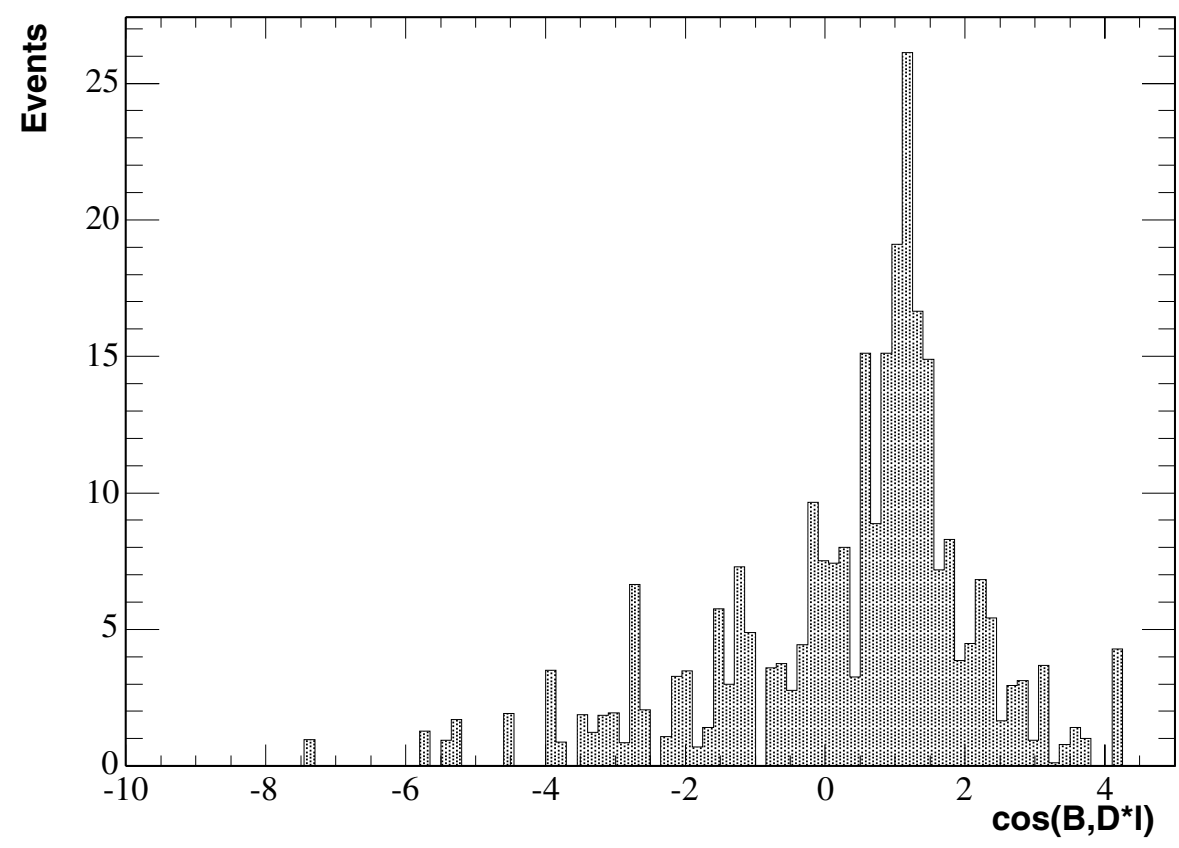

Figure 6.7: $\cos \theta_{B, D^{*} \ell}$ for continuum background taken from generic $c \bar{c}$ MC truth.

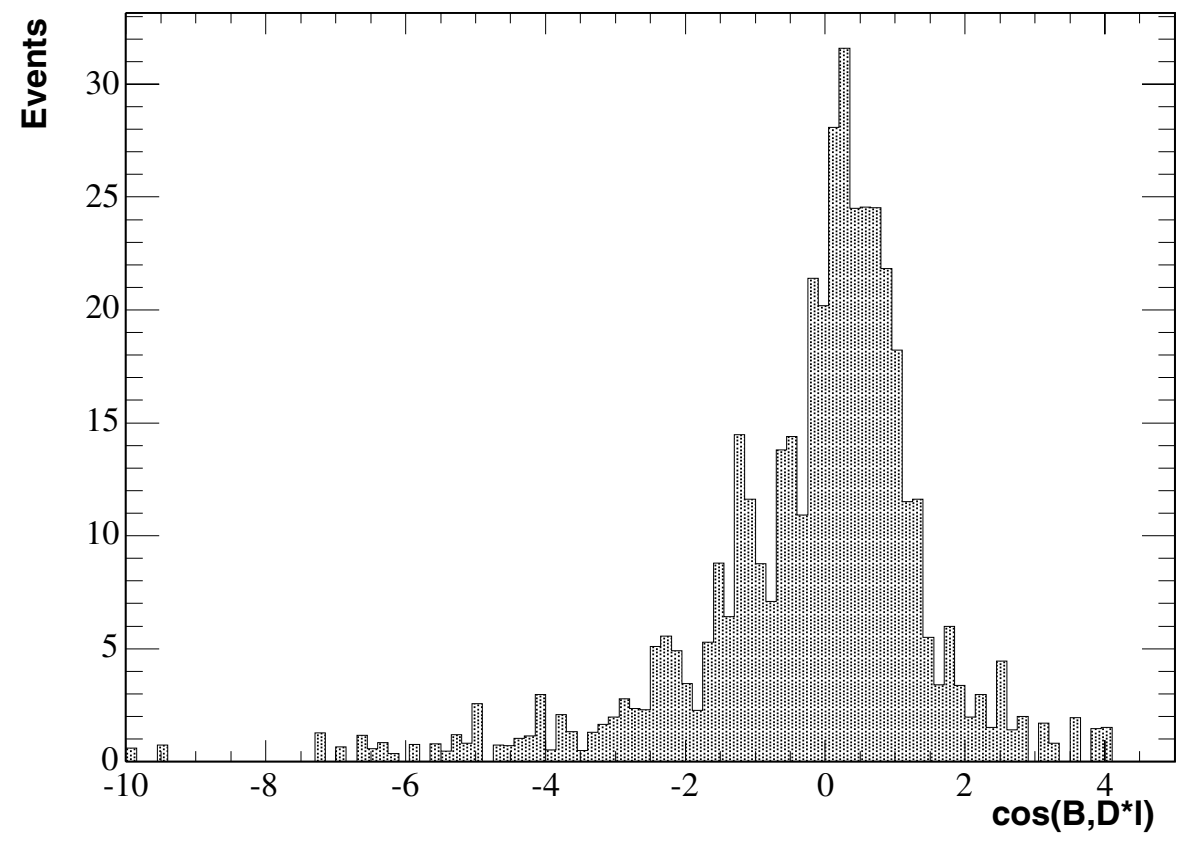

Figure 6.8: $\cos \theta_{B, D^{*} \ell}$ for combinatoric background taken from generic $B^{0}$ and $B^{ \pm} \mathrm{MC}$ truth. 
fails the real $D^{*}$ variable. Since the $D^{*}$ candidates do not represent real $D^{*}$ mesons, there is no real association to a $B$ decay, even if the $B$ meson exists in the event. Thus, the $\cos \theta_{B, D^{*} \ell}$ distribution for the combinatoric background is similar to that of the continuum background, as shown in Figure 6.8.

\subsection{1 $\delta m$ fitting}

The number of combinatoric background events can be determined from the data without relying on the MC simulations. This is generally more reliable since it does not depend on how well the detector and decays are modeled. The method for measuring the combinatoric background relies upon the $\delta m\left(=m_{D^{*}}-m_{D^{0}}\right)$ variable. Real $D^{*}$ s are strongly peaked in the $\delta m=145 \mathrm{MeV} / c^{2}$ mass region. As can be seen in Figure 5.5 , the distribution shows a high signal-to-noise and a well defined background.

This distribution can be modeled with two probability density functions: the signal peak, and the background function. The signal peak is described by a double Gaussian function, with five free parameters: $m_{1}, s_{1}, m_{2}, s_{2}$, and $f$.

$$
\mathcal{F}_{\text {peak }}(\delta m)=\frac{f}{\sqrt{2 \pi} s_{1}} \exp \left(-\frac{\left(\delta m-m_{1}\right)^{2}}{2 s_{1}^{2}}\right)+\frac{1-f}{\sqrt{2 \pi} s_{2}} \exp \left(-\frac{\left(\delta m-m_{2}\right)^{2}}{2 s_{2}^{2}}\right)
$$

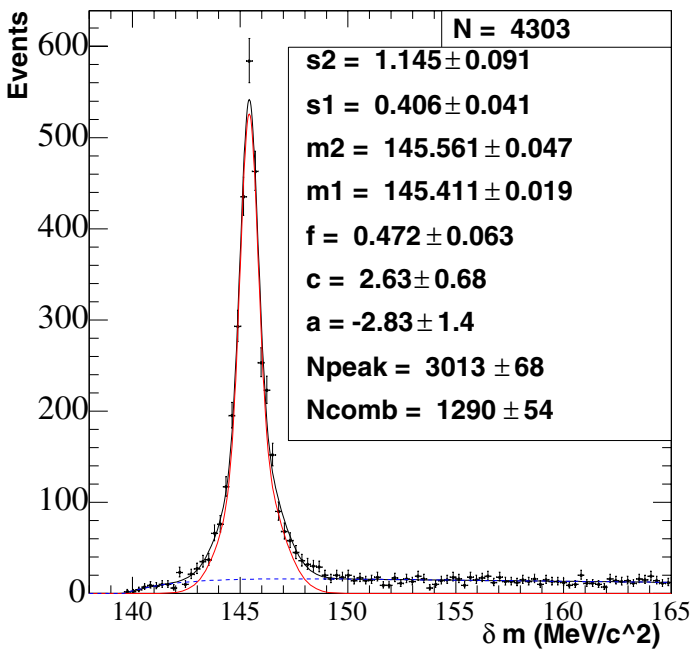

(a) SVT

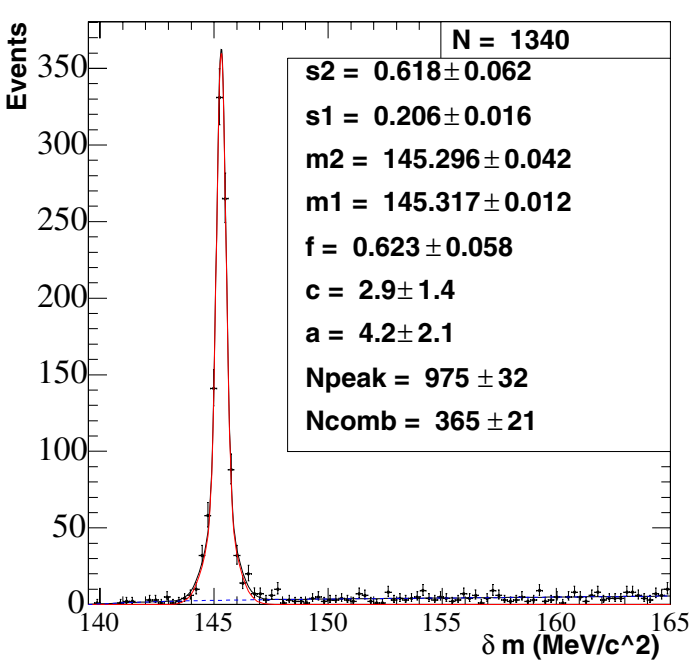

(b) $\mathrm{SVT}+\mathrm{DCH}$

Figure 6.9: Fits to $\delta m$ distribution for peak signal and combinatoric background from on peak data. It has been split into samples depending on whether the slow pion remains in the SVT or enters the DCH. The combinatoric background comes from events in the peaking region below the dashed line. 


\begin{tabular}{|c|c|c|}
\hline Sample & Peak yield & Combinatoric yield \\
\hline SVT & $2946 \pm 66$ & $285 \pm 12$ \\
SVT+DCH & $962 \pm 32$ & $33.0 \pm 2.0$ \\
\hline Total & $3908 \pm 73$ & $318 \pm 12$ \\
\hline
\end{tabular}

Table 6.3: Yields in $\delta m$ peaking region, integrated over the region included by the $\delta m$ cuts for the SVT and SVT+DCH samples.

The shape of the combinatoric background is given by the two parameter function

$$
\mathcal{F}_{\text {comb }}(\delta m)=\frac{1}{N}\left[1-\exp \left(\frac{\delta m-\delta m_{0}}{c}\right)\right]\left(\frac{\delta m}{\delta m_{0}}\right)^{a},
$$

where $N$ is a normalization constant, and $\delta m_{0}$ is the kinematic threshold equal to the pion mass; $a$ and $c$ are the free parameters. The functions are combined together to make an overall PDF:

$$
\mathcal{F}(\delta m)=N_{\text {peak }} \mathcal{F}_{\text {peak }}(\delta m)+N_{\text {comb }} \mathcal{F}_{\text {comb }}(\delta m),
$$

where $N_{\text {peak }}+N_{\text {comb }}$ is constrained to the number of events in the entire sample.

The data are fitted to the PDF using an unbinned, extended, maximum likelihood fit. The fit is performed with the Root analysis program, ${ }^{[58]}$ using the RooFit toolkit ${ }^{[59]}$ as an interface to the Minuit fitting package. ${ }^{[60]}$ Before fitting, the data is split into two categories based on whether or not the slow pion reaches the DCH. With the improved resolution in the $\mathrm{DCH}$, the $\delta m$ peak is narrower than for SVT only tracks and requires separate shape parameters.

After fitting, the PDFs are integrated over the regions included by the $\delta m$ cuts $(\delta m=$ $143-148 \mathrm{MeV} / c^{2}$ for SVT only, and $\delta m=144-147 \mathrm{MeV} / c^{2}$ for SVT+DCH) to determine the number of events in the peaking region. Summing the total number of combinatoric events in the SVT/SVT+DCH categories gives the total number of events in the combinatoric background. As can be seen from Figure 6.9, the fits are in excellent agreement with the data, with good precision in the fitted parameters. The results are summarized in Table 6.3, and show that the total combinatoric background is $318 \pm 12$ events.

\section{Summary of $\bar{B}^{0} \rightarrow D^{*+} \mu^{-} \bar{\nu}_{\mu}$ Signal and Backgrounds}

Careful study of the data and the MC shows that the data set passing the reconstruction and selection criteria contains six different sources of backgrounds as well as the $\bar{B}^{0} \rightarrow$ $D^{*+} \mu^{-} \bar{\nu}_{\mu}$ signal events. The last major step in measuring the $\bar{B}^{0} \rightarrow D^{*+} \mu^{-} \bar{\nu}_{\mu}$ branching fraction is to determine the number of signal events. For consistency, this also requires the determination of the total number of background events. Of the six backgrounds, the number of events in two categories have already been determined: the continuum and combinatoric backgrounds.

By using variables derived from MC truth, the signal and background can each be isolated from the generic MC into mutually exclusive samples. Comparisons of the distributions of the $\cos \theta_{B, D^{*} \ell}$ variable show that the signal sample produces a very distinct 
distribution, and several of the backgrounds also produce distributions which are quite different from each other. These differences allow the MC samples to be fit to the data in order to determine the number of events in the data set corresponding to each sample. 


\section{Chapter 7}

\section{Branching Fraction Extraction}

The $\bar{B}^{0} \rightarrow D^{*+} \mu^{-} \bar{\nu}_{\mu}$ signal and the backgrounds in the data set are understood and represented by the Monte Carlo simulated data. The $\cos \theta_{B, D^{*} \ell}$ variable has been identified which produces distinct distributions for most of the different background types. At this point, a fit can be applied to determine the number of events from signal and from background exist in the data set. With the count of the number of signal events in the data set and the reconstruction efficiency, the $\bar{B}^{0} \rightarrow D^{*+} \mu^{-} \bar{\nu}_{\mu}$ branching fraction can be calculated.

\subsection{Fitting Signal and Background}

\subsubsection{Maximum Log Likelihood Function}

The fit to determine the count of signal and background events is performed on the $\cos \theta_{B, D^{*} \ell}$ distribution for data and MC. The MC is split into the the $\bar{B}^{0} \rightarrow D^{*+} \mu^{-} \bar{\nu}_{\mu}$ signal and the six background modes described in the previous chapter. If the shape of each set is properly modeled, and the scaling on each set matches that of the data, then the sum of events in each bin of the MC histograms will total the number of events in the data histogram.

The predicted number of events from the MC in bin $j$ is given by

$$
f_{j}=\sum_{i} P_{i} a_{i j}
$$

where $i$ represents the seven MC histograms modeling the signal and backgrounds; $a_{i j}$ is the number of events in bin $j$ of histogram $i$, and $P_{i}$ is the scaling factor for the histogram. The $P_{i}$ values are the parameters to be determined by the fit.

From Poisson statistics, the likelihood for observing the number of data events, $n_{j}$, based on prediction $f_{j}$ is given by

$$
\mathcal{L}\left(n_{j} ; f_{j}\right)=\prod_{j} \frac{f_{j}^{n_{j}} e^{-f_{j}}}{n_{j} !},
$$

or

$$
\begin{aligned}
\ln \mathcal{L} & =\sum_{j} \ln \left(\frac{f_{j}^{n_{j}} e^{-f_{j}}}{n_{j} !}\right) \\
& =\sum_{j}\left[n_{j} \ln \left(f_{j}\right)-f_{j}-\ln \left(n_{j} !\right)\right]
\end{aligned}
$$


This function varies depending on the value of the prediction in relation to the data. It reaches a maximum value when $f_{j}=n_{j}$ for all bins. Therefore, the set of parameters, $P_{i}$ which produces the best agreement to the data, also produces the maximum value for the log likelihood function. This is a standard technique for finding the optimal set of parameters for the function in order to match the data.

\subsubsection{The $\chi_{\lambda}^{2}$ Function}

The other standard fitting technique for finding the optimal parameters is the method of least squares. The difference between the prediction, $f_{j}$ and the data $n_{j}$ is given by

$$
\chi^{2}=\sum_{j} \frac{\left(n_{j}-f_{j}\right)^{2}}{\sigma_{j}^{2}},
$$

where $\sigma_{j}=\sqrt{n_{j}}$ is the error on the number of data events in bin $j . \chi^{2}$ becomes zero when the prediction exactly matches the data in each bin. Therefore, the optimal parameters can be determined by minimizing the $\chi^{2}$ value.

Both techniques will provide the central value and confidence interval (uncertainty) for each parameter. The advantage of the least squares method is that the ratio of $\chi^{2} /$ (degrees of freedom) also provides a measure of the goodness-of-fit. The disadvantage of the $\chi^{2}$ fit is that it becomes inaccurate for bins containing few events. In these cases, the Poisson likelihood is necessary. Given that the tails of the $\cos \theta_{B, D^{*} \ell}$ histograms often include bins with very few events, it is not reasonable to use the $\chi^{2}$ distribution.

It is possible to modify the likelihood function in a way which provides a goodnessof-fit. Instead of maximizing the likelihood, one can maximize the likelihood ratio:

$$
\lambda=\frac{\mathcal{L}\left(n_{j} ; f_{j}\right)}{\mathcal{L}\left(n_{j} ; n_{j}\right)} .
$$

This is equivalent to minimizing the quantity

$$
\chi_{\lambda}^{2}=-2 \ln \lambda=2 \sum_{j}\left[\left(f_{j}-n_{j}\right)+n_{j} \ln \frac{n_{j}}{f_{j}}\right] .
$$

Since the denominator of the likelihood ratio is a constant, independent of $f_{j}$, minimizing $\chi_{\lambda}^{2}$ produces the same central value and confidence interval as the standard maximum likelihood fit. Additionally, $\chi_{\lambda}^{2}$ asymptotically obeys a standard chi-square distribution. ${ }^{[62]}$ Therefore, it also provides a goodness-of-fit measurement.

\subsubsection{The Input Histograms}

The predicted number of events based on the MC has seven parameters: one each for the $\bar{B}^{0} \rightarrow D^{*+} \mu^{-} \bar{\nu}_{\mu}$ signal and the six different backgrounds. The $\cos \theta_{B, D^{*} \ell}$ distribution for each sample has been shown in the previous chapter. Of the seven parameters, two can be determined without relying on the fit. As described in Section 6.6, there are $183 \pm 11$ continuum events. The number of combinatoric events is $318 \pm 12$, as shown in Section 6.7. Therefore, the number of fit parameters is reduced to five. 


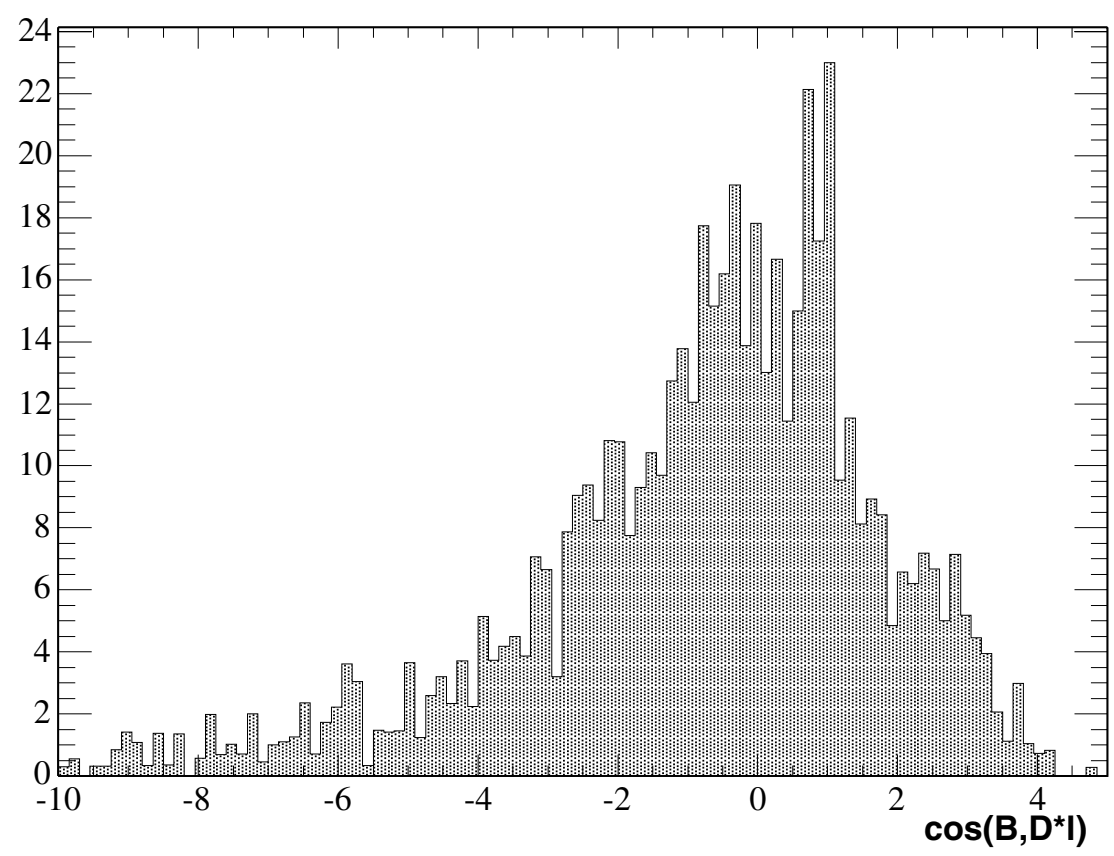

Figure 7.1: $\cos \theta_{B, D^{*} \ell}$ for combined muon backgrounds taken from generic $B^{0}$ and $B^{ \pm}$ MC truth.

In fitting a set of template histograms to the single data histogram, it is important that the distributions for each template are distinct. If two histograms are very similar, it leads to strong correlations between the two fit parameters, and increases the difficulty in finding an optimal parameter set. Comparing the $D^{*+} \mu^{-} \bar{\nu}_{\mu} \pi$ background in Figure 6.3 and the correlated lepton background in Figure 6.4, shows that the two shapes are quite similar.

In order to solve this problem, the three muon backgrounds (correlated, uncorrelated, and fake) are merged together into a single histogram. Given that all three types are backgrounds stemming from aspects of the lepton candidate, treating them as a group is a reasonable choice. The proportions of each of the three types is taken from that modeled by the generic $B \mathrm{MC}$. Since the number of events in the correlated, uncorrelated, and fake MC truth samples are 76, 261, and 221 respectively, the proportion of these backgrounds within the merged lepton background sample is $13.6 \%, 46.8 \%$, and $39.6 \%$, respectively. The resulting $\cos \theta_{B, D^{*} \ell}$ histogram has a very different distribution compared to the other backgrounds, as can be seen in Figure 7.1. An additional benefit of grouping the lepton backgrounds is a further reduction of the of the number of parameters to be fitted down to three.

The main data histogram and the five MC templates, representing $\bar{B}^{0} \rightarrow D^{*+} \mu^{-} \bar{\nu}_{\mu}$ signal and the backgrounds are shown in Figure 7.2. These six histograms are the inputs to the fitting function. The histograms are each normalized at the start of the fitting routines, so the relative number of events in each histogram will not affect the values of 
OnPeak Data

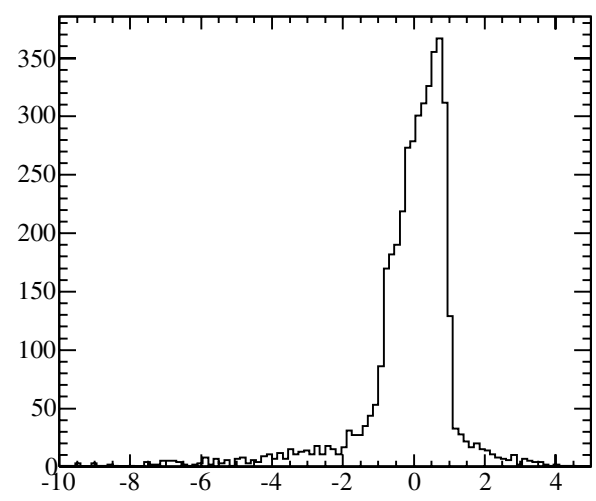

$\mathbf{D}^{*} \mu v \pi \mathbf{B G}$

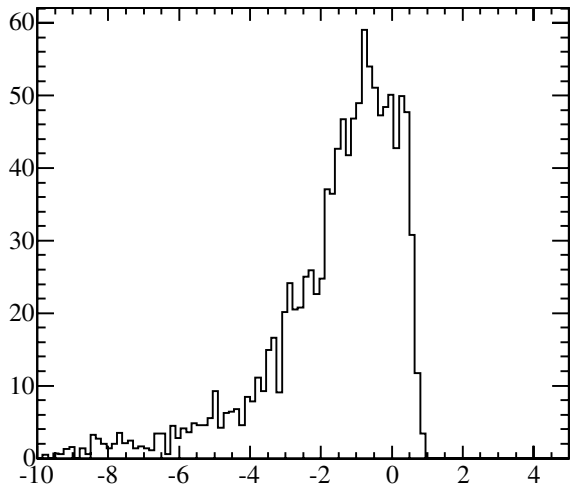

Continuum BG

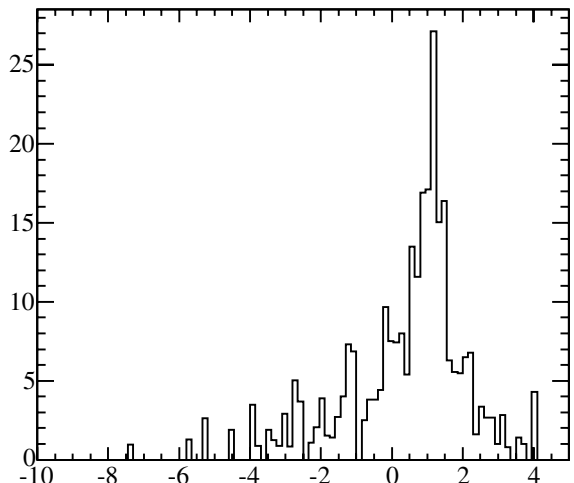

$\mathrm{D}^{\star} \mu \nu$ Signal

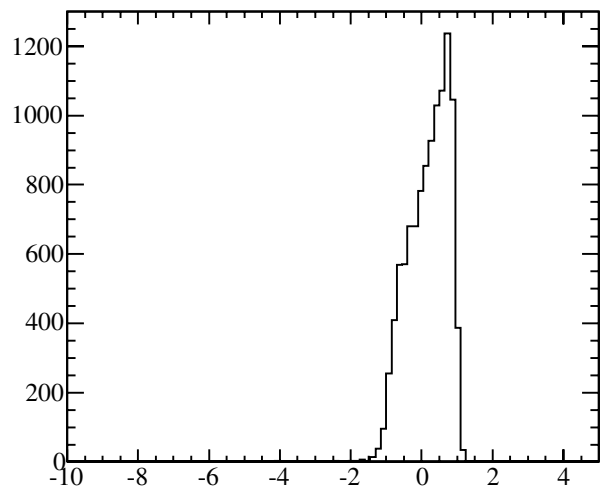

Lepton BG

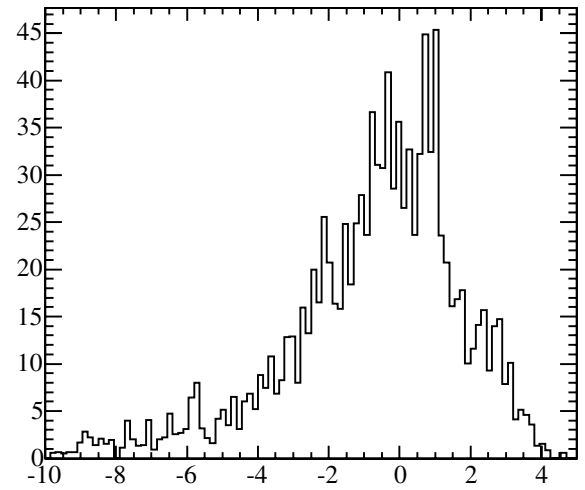

Combinatoric $\mathrm{D}^{*} \mathrm{BG}$

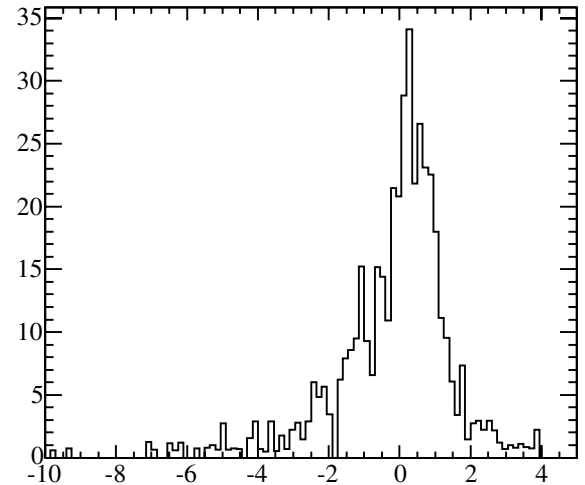

Figure 7.2: $\cos \theta_{B, D^{*} \ell}$ distributions for on-peak data, $\bar{B}^{0} \rightarrow D^{*+} \mu^{-} \bar{\nu}_{\mu}$ signal, and the four background templates. These six histograms make up the inputs to the fit. Each histogram is normalized at the start of the fitting routine, so the relative number of events in each template only affects the statistical uncertainties in each bin. 
the fit parameters. However, the number of events in each bin affects the Poisson error for that bin, which ultimately affects the confidence intervals for the fit parameters. The fitting function is well designed to handle low statistics in the individual bins.

\subsubsection{Root TFractionFitter}

The fit is performed in the Root analysis program using the function known as TFractionFitter. It is specifically designed to perform a binned maximum likelihood fit to determine the optimal scaling parameters for a set of template histograms to fit them to a single data histogram. The inputs to this function are the histograms, as described above, and the output is a single parameter per template. The parameters total to 1 , and each corresponds to the fraction of events in the data histogram which are represented by the particular template. For example, if the parameter for the $\bar{B}^{0} \rightarrow D^{*+} \mu^{-} \bar{\nu}_{\mu}$ signal histogram is 0.75 , it means that $75 \%$ of the events in the data are signal events. Multiplying the parameters by the total number of events in the data histogram produces a count of the number of events for the signal and each type of background.

The TFractionFitter function also includes the ability to properly handle the statistical errors from bins with few events in the MC templates. The maximum likelihood function (Eq. 7.3) and the $\chi^{2}$ function (Eq. 7.4) are based on the statistics for the data histogram alone, without accounting for statistical fluctuations in the MC templates.

In the $\chi^{2}$ function, one can adjust the value of $\sigma_{j}^{2}$ to include the sum of the Gaussian errors for each component, instead of just the data. However, this would still be inaccurate for bins with few events. The maximum likelihood function is not so easy to modify to add the Poisson statistics of the MC templates, but it can be done.

In order to fix the maximum likelihood function, an additional likelihood must be introduced. ${ }^{[63]}$ For each template, in each bin, there is an expected number of events, $A_{i j}$ for which $a_{i j}$ is the observation. The prediction for the number of data events in each bin, Eq. 7.1, should therefore be

$$
f_{j}=\sum_{i} P_{i} A_{i j} .
$$

The total likelihood is now the combined probability of the observed data, $n_{j}$, and the observed MC, $a_{i j}$.

$$
\ln \mathcal{L}=\sum_{j}\left[\left(n_{j} \ln f_{j}-f_{j}\right)+\sum_{i}\left(a_{i j} \ln A_{i j}-A_{i j}\right)\right] .
$$

Maximizing this likelihood gives estimates for the parameters $P_{i}$ and $A_{i j}$. Converting this to the $\chi_{\lambda}^{2}$ function gives

$$
\chi_{\lambda}^{2}=2 \sum_{j}\left[f_{j}-n_{j}+n_{j} \ln \frac{n_{j}}{f_{j}}+\sum_{i}\left(A_{i j}-a_{i j}+a_{i j} \ln \frac{a_{i j}}{A_{i j}}\right)\right] .
$$

The effect of including $A_{i j}$ into the fit is to slightly modify the shape of each template within the Poisson uncertainties for each bin. This helps to correct for statistical fluctu- 
ations in the MC templates due to low numbers of events in some bins. It also causes the overall prediction to be different than a simple linear sum of the template histograms.

The TFractionFitter function uses this treatment to handle the statistics in the MC templates. Furthermore, it calculates the estimates for each $A_{i j}$ analytically, instead of treating each value as a separate fit parameter. The data and MC histograms, and the $A_{i j}$ predictions are then used as inputs to the Minuit fitting software to determine the best estimates for the parameters $P_{i}$.

Minuit is a standard tool for minimizing any function by varying a set of parameters. ${ }^{[60]}$ It has been in use in the high-energy physics community for many years. It handles the determination of the central values and confidence intervals very well. It also calculates the correlation matrix between the various parameters, and it can find non-parabolic errors on the central values.

One adjustment was made to the TFractionFitter function provided by Root. The maximum log likelihood function normally used, Eq. (7.8) was replaced by the $\chi_{\lambda}^{2}$ function of Eq. (7.9). In making this change, the fit was tested repeatedly to demonstrate that the $\chi_{\lambda}^{2}$ fit did produce the same central value and confidence interval for each parameter. Additionally, a method was added to calculate the number of degrees of freedom for the fit. This is defined as the number of non-zero bins in the data set minus the number of fit parameters, $P_{i}$.

By using the TFractionFitter function with the histogram inputs shown in Figure 7.2 , a good fit to the data is achieved, which yields a count of the number of signal and background events and the errors on these quantities.

\section{$7.2 \quad$ Fit Results}

The results of the fit are shown in Figure 7.3. The MC templates, scaled by the fractions returned from the fit, add together to match the data quite well throughout the distribution. The fractions for each MC template are listed in Table 7.1.

\begin{tabular}{|c|c|c|}
\hline Type & Fraction (\%) & Number of Events \\
\hline $\bar{B}^{0} \rightarrow D^{*+} \mu^{-} \bar{\nu}_{\mu}$ signal & $68.6 \pm 1.7$ & $2911 \pm 73$ \\
$D^{*+} \mu^{-} \bar{\nu}_{\mu} \pi$ background & $8.7 \pm 1.9$ & $368 \pm 81$ \\
muon background & $11.0 \pm 2.0$ & $467 \pm 86$ \\
continuum background & $4.31 \pm 0.26$ & $183 \pm 11$ \\
combinatoric background & $7.50 \pm 0.28$ & $318 \pm 12$ \\
\hline data & 100 & 4243 \\
\hline
\end{tabular}

Table 7.1: The results of the main fit. The fractions for each parameter are multiplied by the number of events in the data to determine the number of events in the signal or backgrounds. 


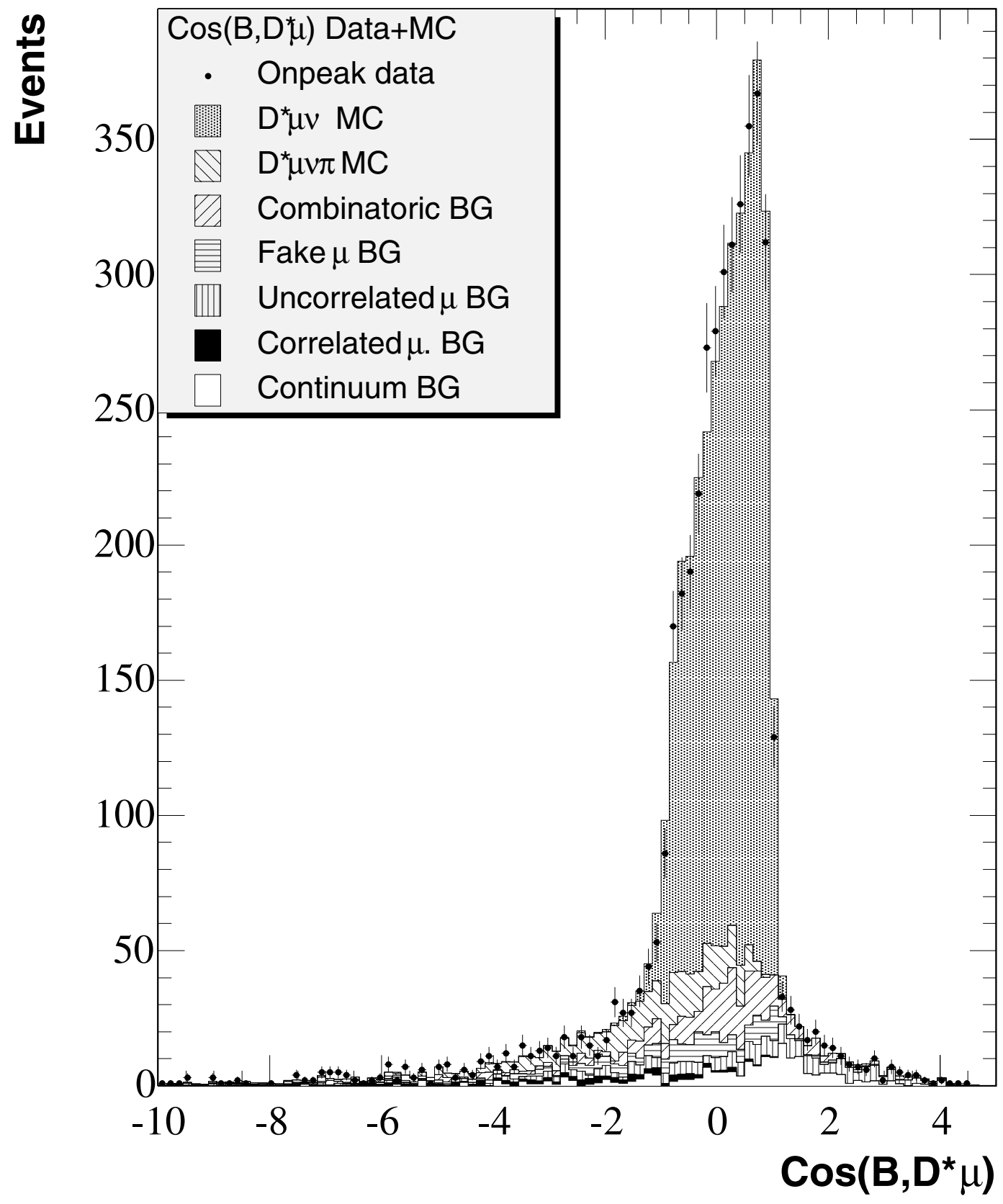

Figure 7.3: Fit Results. The data points are overlayed on the sum of the MC template histograms. The templates shown are the predicted templates after the fit has completed, scaled by the fractions returned by the fit. The three muon backgrounds are taken from the individual histograms before they were merged for the fit, but all are scaled by the muon background fraction. 
The correlation matrix for the three fitted parameters is

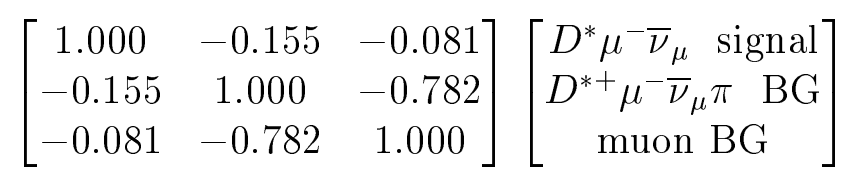

The correlation is fairly high between the muon and $D^{*+} \mu^{-} \bar{\nu}_{\mu} \pi$ background. However, the correlations between the signal and the backgrounds is reasonably low. Since the actual number of events in each background mode is not important, this looks sufficient.

It is interesting to examine the predicted template histograms returned by the fit. As described above, the TFractionFitter code can modify the contents of each bin in the templates within their statistical uncertainties. This can be seen in Figure 7.4. The adjustments from the input quantities are almost always less than $\frac{1}{2} \sigma$. Thus, the effect is fairly small and entirely within the MC statistics.

Careful observation of the combinatoric background plot in Figure 7.4 shows an area where care must be taken in using this treatment of the template histograms. At the left and right edges of this histogram are a couple of bins where zero events exist in the input template, but the prediction shows a non-zero value. This is statistically reasonable, but it can lead to unreasonable predictions in cases of very low statistics where many of the bins are empty. ${ }^{\text {a }}$ Therefore, one must be careful to check the predictions when the bins are empty in the input templates. In this case, there are only a couple of bins at the outer edges of the histogram, so there is no problem.

The $\chi_{\lambda}^{2}=105$ for 86 degrees of freedom, yielding a $\chi^{2}$ per degree of freedom of 1.2 . This corresponds to a $\chi^{2}$ probability of $7.9 \%$, which is a reasonable value. Thus, the fit results show good mathematical agreement with the data.

Another useful check on the quality of the fit is the histogram of the pulls:

$$
\text { pull }_{j}=\frac{\text { data }_{j}-\text { prediction }_{j}}{\sigma_{j}}
$$

where $\sigma_{j}$ is the error in bin $j$ for the data histogram. Ideally, the pulls have a Gaussian distribution centered at 0 , with a width of 1 . Figure 7.5 shows the differences in each bin and the pulls for the fit. The pulls agree moderately well to the ideal distribution within the statistics of the histogram, although the spread looks somewhat low. However, given that there are only 100 bins being fitted, the statistics of the pulls is small.

\subsubsection{Efficiency}

The $2911 \pm 73$ signal events obtained from the fit is not a count of the number of $\bar{B}^{0} \rightarrow$ $D^{*+} \mu^{-} \bar{\nu}_{\mu}$ decays in the data. This is only a count of the number of signal events that entered the data set. When the criteria for selecting the desired events are defined, it is inevitable that some signal events are going to fail the criteria and not enter the data set.

\footnotetext{
${ }^{\mathrm{a}}$ In the analytical method to determine the predicted template shapes (see Section 7.1.4), only the histogram with the smallest area is subject to changes in bins with zero events. ${ }^{[63]}$
} 
$\mathbf{D}^{\star} \mu \nu$ Signal

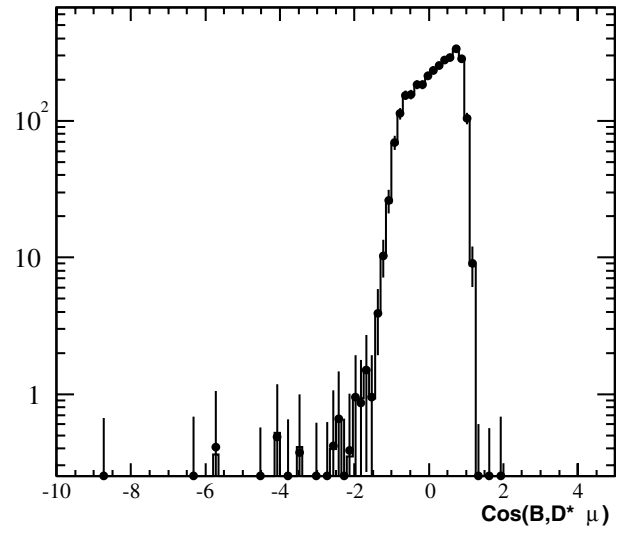

Lepton BG

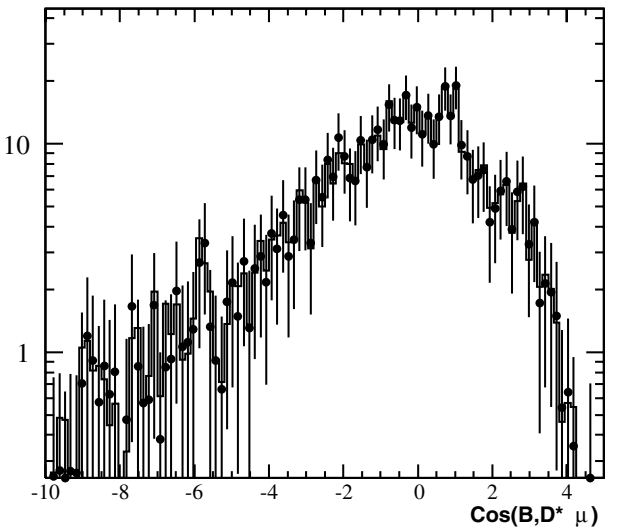

\section{$\mathbf{D}^{\star} \mu v \pi \mathbf{B G}$}

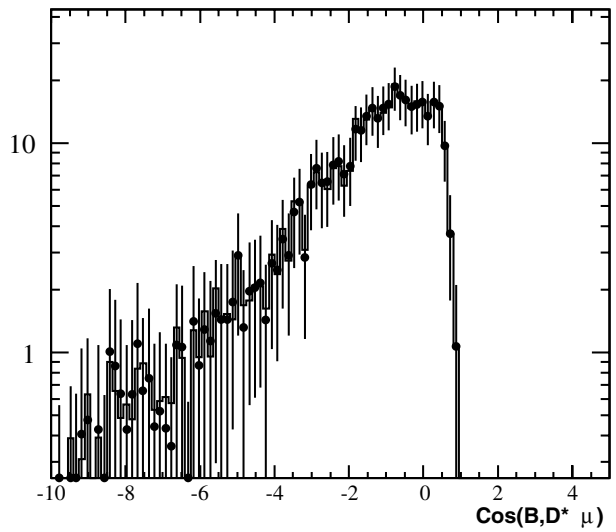

Continuum BG

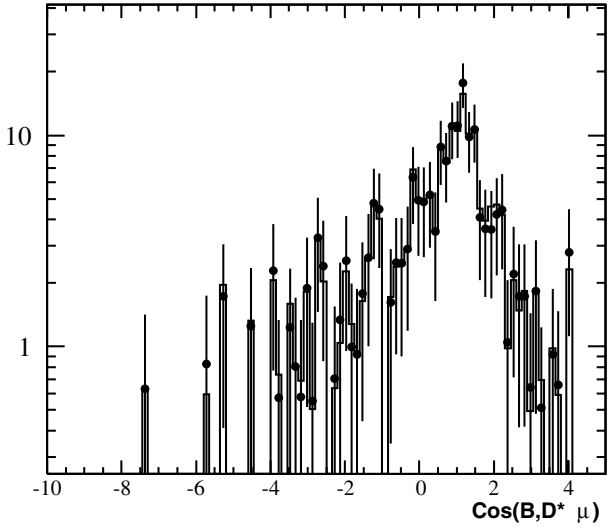

Combinatoric BG

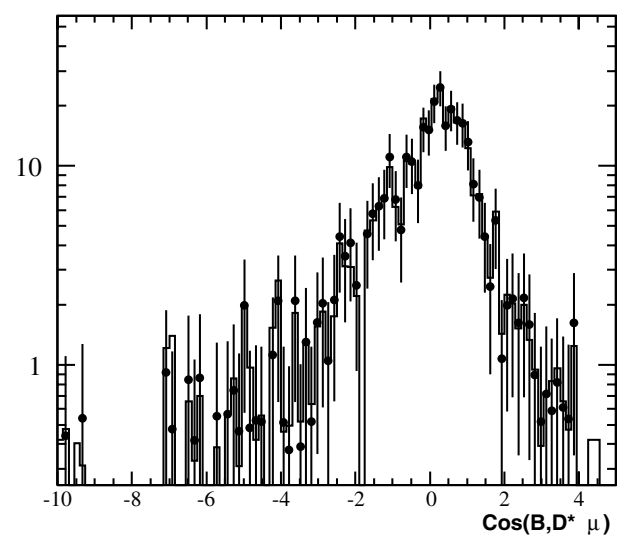

Figure 7.4: Comparing the predicted templates (line) with the input (points) for the five MC histograms used in the TFractionFitter function. The adjustments made for the predicted templates are almost always less than $\frac{1}{2} \sigma$ for the statistical fluctuations of the bins. 


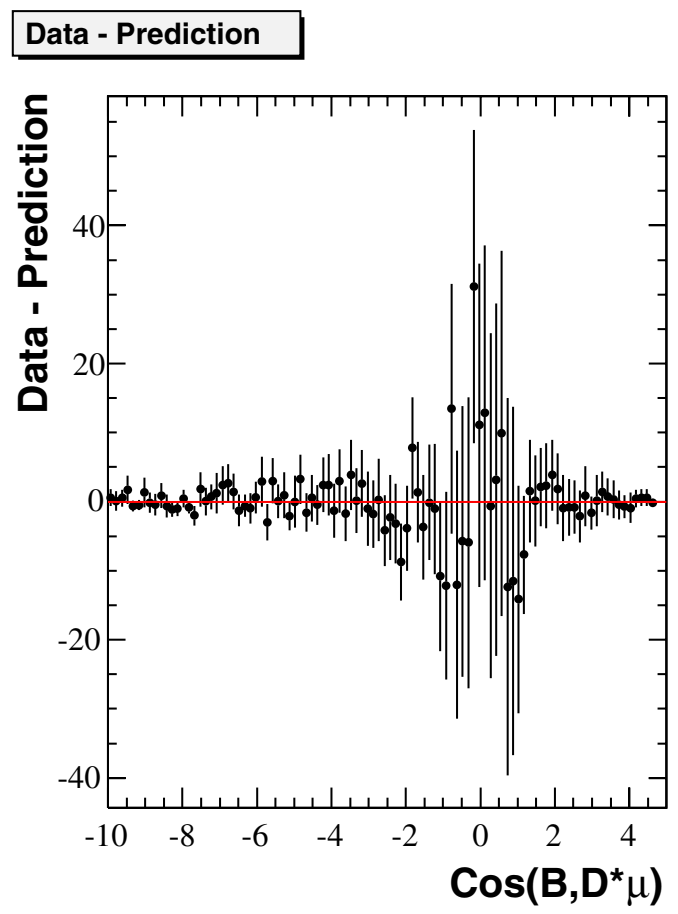

\section{Pulls}

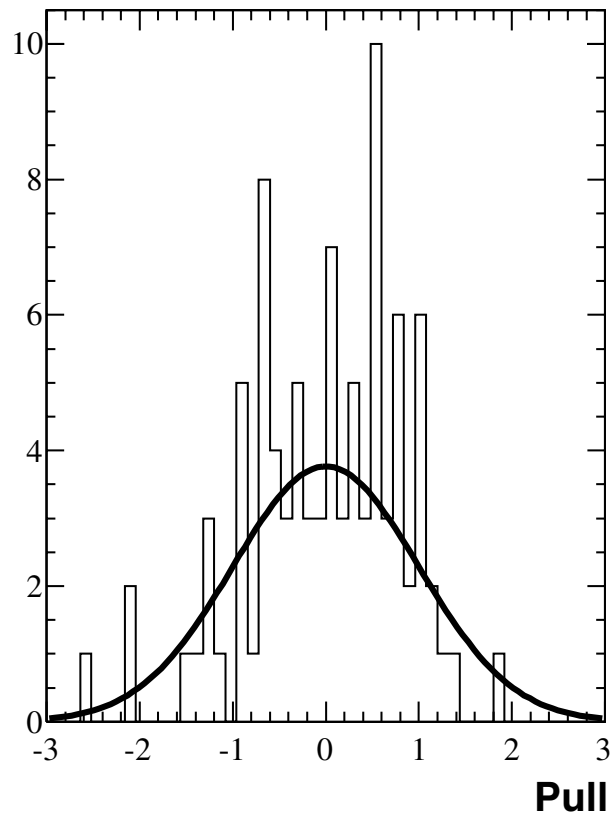

Figure 7.5: The left plot shows the difference between the data and the prediction in each bin. The pulls are shown in the right plot. A Gaussian distribution of width 1 is fit to the pulls to show an ideal result.

A correction must be applied to account for these missing events. The reconstruction efficiency, $\epsilon$ must be determined in order to get the proper number.

The efficiency can be found by using the $\bar{B}^{0} \rightarrow D^{*+} \ell^{-} \bar{\nu}_{\ell}\left(D^{0} \rightarrow K^{-} \pi^{+}\right)$singlemode MC. Each event in this single-mode MC sample is the specific decay in question. Therefore, there are a known number of decays to be reconstructed. The efficiency is simply the ratio of the number of these decays passing all the selection criteria to the number generated. The $\bar{B}^{0} \rightarrow D^{*+} \ell^{-} \bar{\nu}_{\ell}$ signal MC sample consists of 62,000 events split evenly between electron and muon modes.

In order to reduce the statistical uncertainty and verify the contents of the $\mathrm{MC}$, the exact number of muon mode events is counted from the MC truth table. The decay of each $B$ in the event is examined to see if it is a $\bar{B}^{0} \rightarrow D^{*+} \mu^{-} \bar{\nu}_{\mu}\left(D^{0} \rightarrow K^{-} \pi^{+}\right)$decay. Of the 62,000 events, there are 30,923 of these decays, which is entirely consistent with statistical fluctuations in a $50 \%$ split between electrons and muons.

The number of $\bar{B}^{0} \rightarrow D^{*+} \mu^{-} \bar{\nu}_{\mu}$ events reconstructed from the signal MC is $3412 \pm 58$. This corresponds to an efficiency of $\epsilon=11.03 \pm 0.18 \%$. Therefore, the corrected number of $\bar{B}^{0} \rightarrow D^{*+} \mu^{-} \bar{\nu}_{\mu}$ with $D^{0} \rightarrow K^{-} \pi^{+}$decays is

$$
\frac{N_{\text {observed }}}{\epsilon}=\frac{2911 \pm 73}{0.1103 \pm 0.0018}=26390 \pm 790 .
$$




\subsubsection{Calculating the Branching Fraction}

With the number of decays measured, the branching fraction can be calculated directly. It is a function of the number of $B^{0}$ events analyzed and the branching fractions for the other decays used in the analysis (see Section 5.1).

$$
\begin{aligned}
\mathcal{B}\left(\bar{B}^{0} \rightarrow D^{*+} \mu^{-} \bar{\nu}_{\mu}\right)= & \frac{N_{\text {observed }}}{\epsilon} \frac{1}{N_{\Upsilon(4 S)} 2 \mathcal{B}\left(\Upsilon(4 S) \rightarrow B^{0} \bar{B}^{0}\right)} \\
& \times \frac{1}{\mathcal{B}\left(D^{*+} \rightarrow D^{0} \pi^{+}\right) \mathcal{B}\left(D^{0} \rightarrow K^{-} \pi^{+}\right)}
\end{aligned}
$$

where $N_{\text {observed }}$ is the number of $\bar{B}^{0} \rightarrow D^{*+} \mu^{-} \bar{\nu}_{\mu}$ observed in the data set, and $N_{\Upsilon(4 S)}$ is the number of $\Upsilon(4 S)$ events in the Run 1 data. The factor of 2 in the denominator accounts for the fact that there are two $B$ mesons per event.

\begin{tabular}{|l|c|}
\hline Parameter & \multicolumn{1}{c|}{ Value } \\
\hline$N_{\text {observed }}$ & $2911 \pm 73$ \\
$\epsilon$ & $11.03 \pm 0.18 \%$ \\
$N_{\Upsilon(4 S)}{ }^{[32]}$ & $(22.32 \pm 0.25) \times 10^{6}$ \\
$\mathcal{B}\left(\Upsilon(4 S) \rightarrow B^{0} \bar{B}^{0}\right)^{[64]}$ & $48.7 \pm 1.3 \%$ \\
$\mathcal{B}\left(D^{*+} \rightarrow D^{0} \pi^{+}\right)^{[6]}$ & $67.7 \pm 0.5 \%$ \\
$\mathcal{B}\left(D^{0} \rightarrow K^{-} \pi^{+}\right)^{[6]}$ & $3.80 \pm 0.09 \%$ \\
\hline
\end{tabular}

Table 7.2: Values entering final $\mathcal{B}$ calculation

The values of these factors are shown in Table 7.2. The value of $\mathcal{B}\left(\Upsilon(4 S) \rightarrow B^{0} \bar{B}^{0}\right)=$ $48.7 \pm 1.3 \%$ is taken from the measured ratio of the $\Upsilon(4 S)$ branching fractions, $\mathcal{B}(\Upsilon(4 S) \rightarrow$ $\left.B^{+} B^{-}\right) / \mathcal{B}\left(\Upsilon(4 S) \rightarrow B^{0} \bar{B}^{0}\right)=1.072 \pm 0.058 .{ }^{[64]}$ This value has been corrected with a newer $B$ lifetime ratio $\tau_{B^{+}} / \tau_{B^{0}}=1.083 \pm 0.017$ measured by $B A B A R^{[65]}$ producing a branching fraction ratio of $1.055 \pm 0.055$.

Therefore, the branching fraction for the $\bar{B}^{0} \rightarrow D^{*+} \mu^{-} \bar{\nu}_{\mu}$ decay is $4.72 \pm 0.12 \%$. The error cited here is only the statistical error derived from the fit. The errors on the other values are systematic errors, and will be included in the general systematic error described in the next chapter.

At this point, in order to determine $\left|V_{c b}\right|$, the data would be broken into bins of the recoil variable, $w$, and the branching fraction would be measured in each bin using this analysis method. However, the scope of this analysis does not cover the $\left|V_{c b}\right|$ measurement. There is a separate group in BABAR which is measuring $\left|V_{c b}\right|$ through the $\bar{B}^{0} \rightarrow D^{*+} \ell^{-} \bar{\nu}_{\ell}$ decay. 


\section{Chapter 8}

\section{Validation and Systematic Studies}

Having measured the $\bar{B}^{0} \rightarrow D^{*+} \mu^{-} \bar{\nu}_{\mu}$ branching fraction to be $4.72 \pm 0.12 \%$, there remains the question of how reliable is the measurement. The reliability of the analysis method and of the MC simulation must be checked in order to be fully confident of the result. With the strong reliance on Monte Carlo simulated data in this analysis, it is important to verify that the MC produces spectra which matches the data. Studies verifying the agreement between $\mathrm{MC}$ and data for the spectra of the muon, slow pion, and the $D^{*}$ are shown. Several additional studies have been made to validate the result and show that the result is not highly sensitive to small changes in the analysis procedure.

The error in the $\bar{B}^{0} \rightarrow D^{*+} \mu^{-} \bar{\nu}_{\mu}$ branching fraction measurement listed above is only a statistical error. With any experiment, there are also systematic errors which must be measured to determine the accuracy of the result. This chapter will describe the various systematic uncertainty studies performed on both the data and the simulations. The systematic errors can come from three main sources: errors from the techniques used in this analysis, errors common to all BABAR collaborators, and errors in branching fraction values measured by other experiments. The systematic uncertainties in the $\bar{B}^{0} \rightarrow D^{*+} \mu^{-} \bar{\nu}_{\mu}$ branching fraction are summarized in Table 8.11 at the end of this chapter.

\subsection{Validation Studies}

\subsubsection{Spectra Comparisons}

An important comparison to verify that the $\mathrm{MC}$ is a good simulation of the data is to compare the spectra of the key particles in the event. The momentum vectors $(p, \theta, \phi)$ in the lab frame for the muon and the slow $\pi$ are compared, and the CM momentum of the $D^{*}$. The MC used is a merged set of $B^{0}, B^{ \pm}$, and $c \bar{c}$ generic MC. Each type has been scaled for the relative luminosity of the sample compared to the on-peak data luminosity. The MC has also had all of the corrections described in Section 5.5 applied.

Additionally, a correction has been applied for the $\bar{B}^{0} \rightarrow D^{*+} \mu^{-} \bar{\nu}_{\mu}$ branching fraction.

In the file defining all the branching fractions for the generic $\mathrm{MC}, \mathcal{B}\left(\bar{B}^{0} \rightarrow D^{*+} \mu^{-} \bar{\nu}_{\mu}\right)$ was set at $5.6 \%$. This is considered to be an error in the MC definitions, and it shows up in many $B A B A R$ analyses involving the $\bar{B}^{0} \rightarrow D^{*+} \mu^{-} \bar{\nu}_{\mu}$ (both as a signal or a background) as a surplus of events in the MC. To correct for this, the $B^{0}$ events are reweighted by $4.7 / 5.6$; the ratio of the measured $\mathcal{B}$ over the defined $\mathcal{B}$.

As can be seen in Figures 8.1 - 8.3, the agreement is very good in all of the plots within the statistical errors of the data and MC sets. Therefore, the MC simulated data 


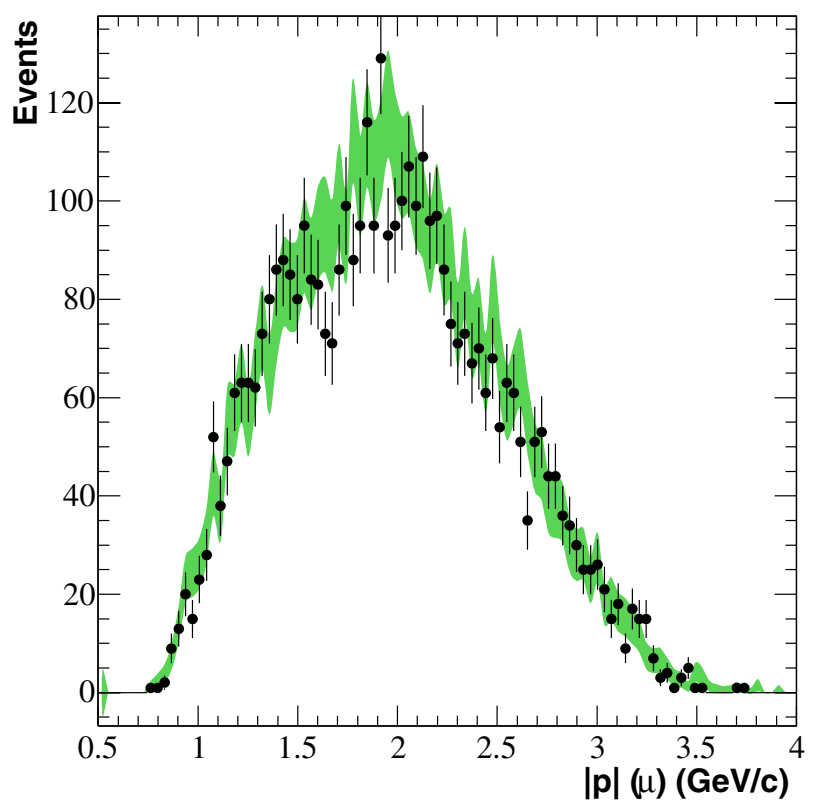

(a) $p(\mathrm{GeV} / c)$

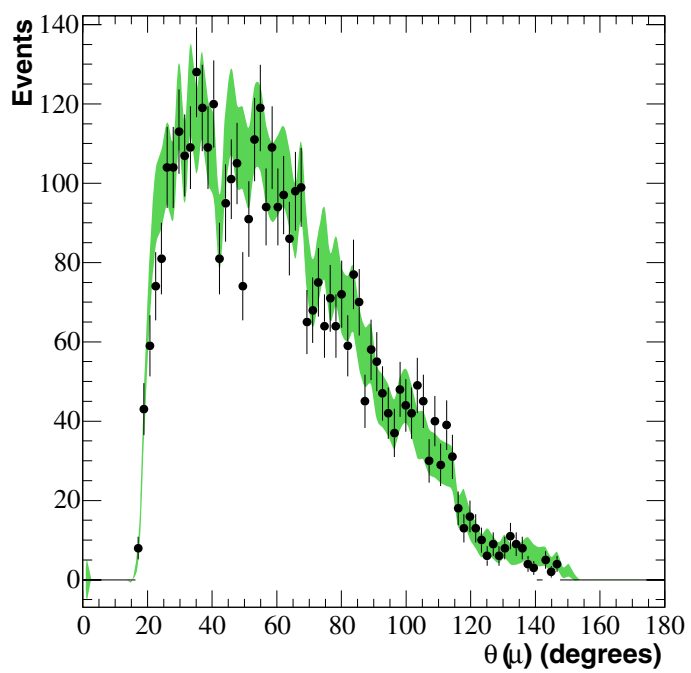

(b) $\theta\left(^{\circ}\right)$

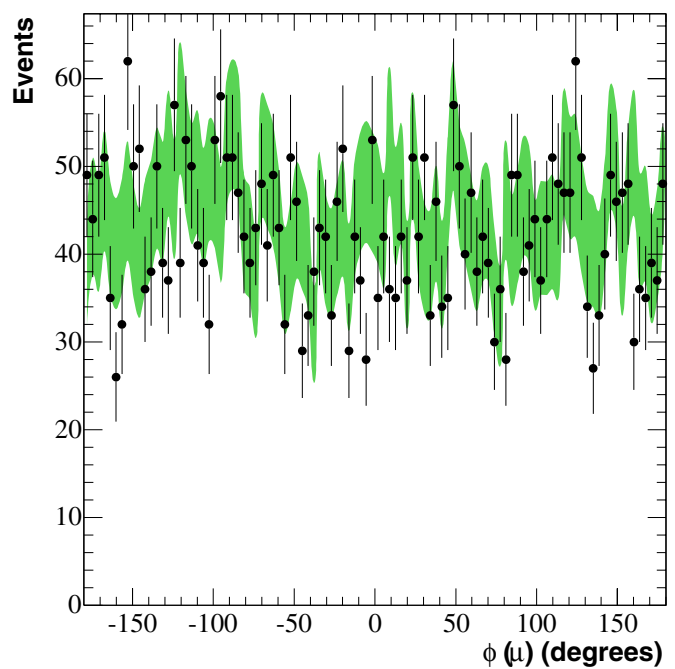

(c) $\phi\left(^{\circ}\right)$

Figure 8.1: Comparisons of muon spectra for data and $\mathrm{MC}$ in the lab frame. The data is shown by the points. The shaded band represents the $1 \sigma$ contour for the generic MC. 


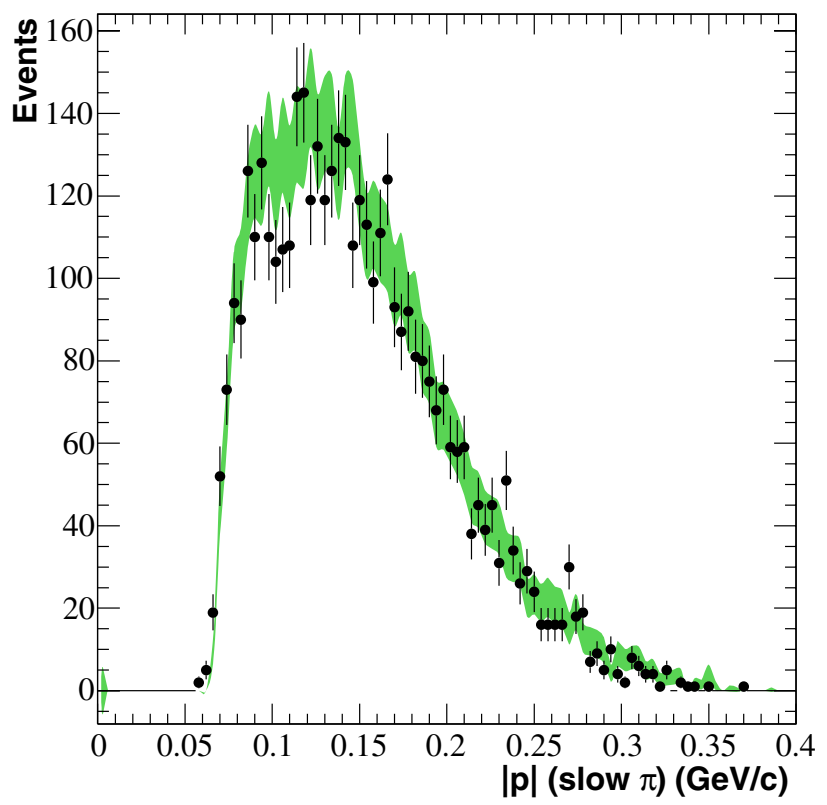

(a) $p(\mathrm{GeV} / c)$

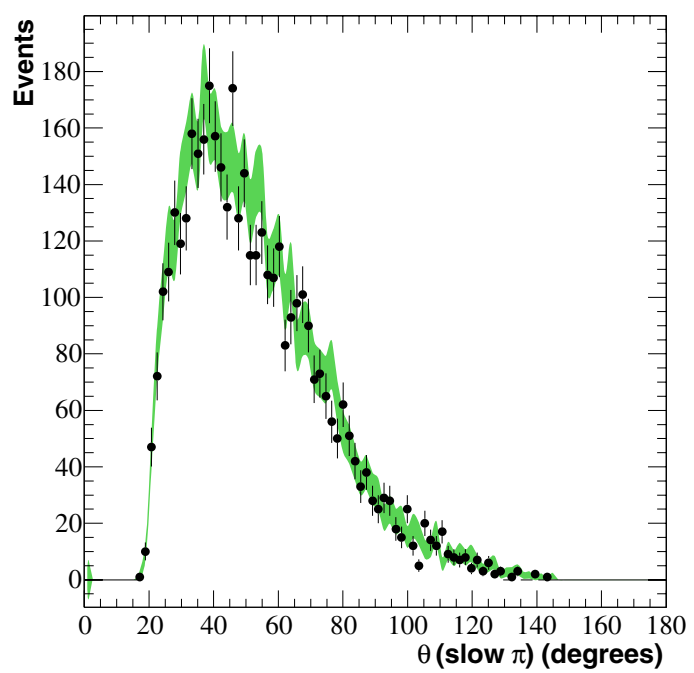

(b) $\theta\left(^{\circ}\right)$

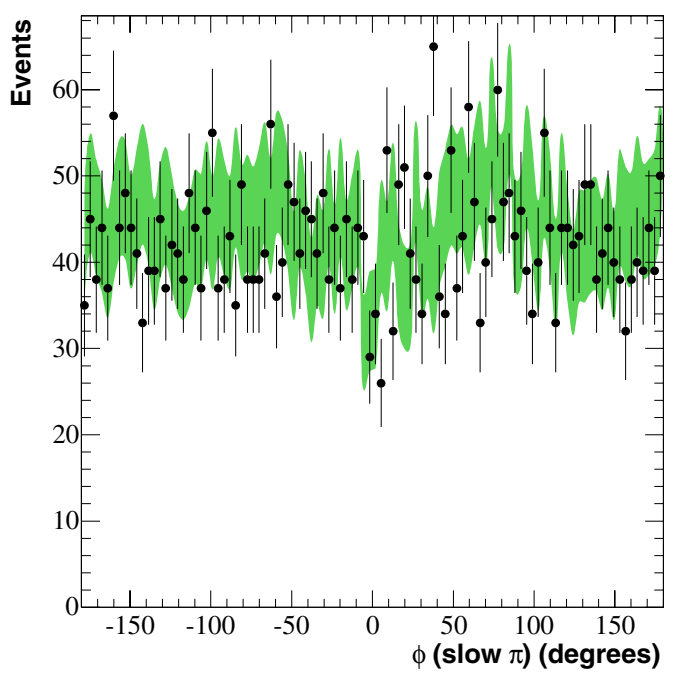

(c) $\phi\left(^{\circ}\right)$

Figure 8.2: Comparisons of slow $\pi$ spectra for data and MC in the lab frame. The data is shown by the points. The shaded band represents the $1 \sigma$ contour for the generic MC. 


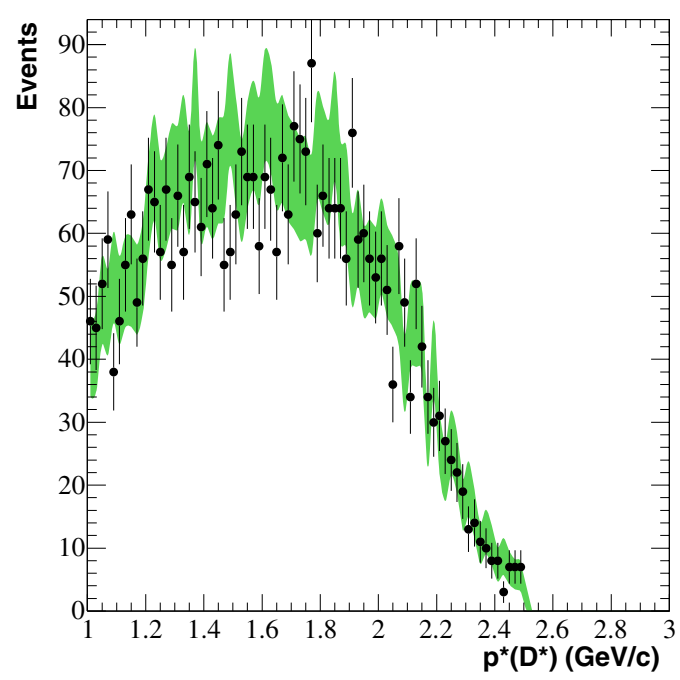

Figure 8.3: Comparisons of $D^{*}$ momentum in the CM frame for data and MC. The data is shown by the points. The shaded band represents the $1 \sigma$ contour for the generic MC.

provides a reliable model for the data.

\subsubsection{Vertexing Algorithm}

The vertexing algorithm is described in Section 5.4.4. It simultaneously fits the muon, the beam spot, the $D^{*}$, and its daughters to find the best vertices. This can dramatically improve the $\delta m$ resolution. Given that this is primarily a refit for the $D^{*}$ and $D^{0}$ vertices, the choice of including constraints on the muon and the beam spot are examined for their systematic effects. The impact is expected to be low in this analysis.

In order to see the dependence of the $\delta m$ mass resolution on the choice of vertexing algorithms, the variables $\delta m$ and $\sigma_{\delta m}$ are recalculated using a two different refitting techniques: one with the muon constraint removed, and the other with the beam-spot constraint removed. The difference would show up in the number of combinatoric events found in the $\delta m$ fit, and therefore could affect the fit to find the number of $\bar{B}^{0} \rightarrow D^{*+} \mu^{-} \bar{\nu}_{\mu}$ signal and background events.

The larger difference from Table 8.1 is only $0.11 \%$, which is negligible. Thus, the vertexing algorithm does not contribute significantly to the systematic error.

\begin{tabular}{|l|c|c|c|c|}
\hline Algorithm & $\begin{array}{c}\text { Number of Comb. } \\
\text { BG Events }\end{array}$ & $\mathcal{B}(\%)$ & $\delta \mathcal{B}(\%)$ & $\delta \mathcal{B} / \mathcal{B}(\%)$ \\
\hline No muon constraint & $317 \pm 12$ & 4.726 & 0.005 & 0.11 \\
No beam-spot constraint & $318 \pm 12$ & 4.722 & 0.001 & 0.02 \\
\hline
\end{tabular}

Table 8.1: Effects of changing constraints in the vertexing algorithm. 


\subsubsection{Histogram Binning for $\mathcal{B}$ Fit}

The number of bins for the histogram from $\cos \theta_{B, D^{*} \ell}=[-10,5]$ is 100 . This number was chosen so as to provide enough bins for reasonable statistics without producing too many empty bins. To see the effect of the bin sizes, the fit is performed on histograms with different numbers of bins. The total range in the $\mathcal{B}$ values from Table 8.2 is only $0.016 \%$. This range is much smaller than the statistical error in the measurement. Therefore, the binning of the $\cos \theta_{B, D^{*} \ell}$ histogram has a negligible impact on the result.

\begin{tabular}{|c|c|}
\hline Num. Bins & $\mathcal{B}(\%)$ \\
\hline 50 & $\mathbf{4 . 7 2 8}$ \\
75 & 4.713 \\
100 & 4.721 \\
125 & 4.718 \\
150 & $\mathbf{4 . 7 1 2}$ \\
175 & 4.715 \\
200 & 4.719 \\
250 & 4.723 \\
\hline
\end{tabular}

Table 8.2: Results of fitting uses different bin sizes. The highlighted values show the maximum and minimum values used to find the range.

\subsubsection{Confirming the Fit Method}

A good test to confirm that the fit to the $\cos \theta_{B, D^{*} \ell}$ histograms produces the appropriate results is to replace the data with the MC in the fit. This provides a sample in which the $\bar{B}^{0} \rightarrow D^{*+} \mu^{-} \bar{\nu}_{\mu}$ branching fraction is known in advance. A merged set of $B^{0}, B^{ \pm}$, and $c \bar{c}$ generic $\mathrm{MC}$, scaled to the relative luminosities of the samples is used to represent the data. The defined $\bar{B}^{0} \rightarrow D^{*+} \mu^{-} \bar{\nu}_{\mu}$ branching fraction is $5.6 \%$. The results of the fit should produce this known value if all is working as expected.

The value returned by the fitting routine yields a branching fraction of $5.61 \pm 0.13 \%$. This is in excellent agreement with the defined branching fraction for the MC. Thus, the fitting method does return the expected value, and can be trusted to return the appropriate value from the data.

\subsection{MC and Data Statistics}

The errors on the fit parameters are a combination of the statistical errors in the data histogram and the $\mathrm{MC}$ templates. In order to isolate the contribution from the $\mathrm{MC}$ statistics, we can scale the size of the data set by several orders of magnitude. The effect of this is to reduce the data statistics within the fit to zero. The resulting error in the fit parameters is then strictly produced by the MC statistics. 
This effect has been studied using toy MC based on Gaussian PDFs. Three single Gaussian distributions are fit to a linear sum of the three Gaussian distributions. The data for each sample is generated randomly based on the given PDF. The coefficients for the linear sum are found readily by the fit, and the statistical error in those fit parameters is clearly dependent on the size of the samples used, both for the individual Gaussian samples and the linearly combined sample. If the number of events generated for the combined sample is increased by orders of magnitude greater than that of the individual samples, the error in the fit parameters drops to a lower limit which represents the statistical error in the individual samples.

In order to test if scaling the size of the data sample will isolate the MC statistics, instead of generating more events in the combined sample, an equal number of events is generated for each sample. The combined sample is then scaled by several orders of magnitude. Just as in the case of generating more events, the error in the fit parameters drops to the same lower limit, representing the statistical error in the individual samples. Therefore, scaling the data set is an effective strategy.

With the MC statistical error isolated, the statistical error can be obtained by subtracting in quadrature the MC statistical error from the total. The errors are summarized in Table 8.3.

\begin{tabular}{|l|c|c|}
\hline Error & $\sigma_{\mathcal{B}}(\%)$ & $\sigma_{\mathcal{B}} / \mathcal{B}(\%)$ \\
\hline Total stat. error (fitted) & 0.12 & 2.5 \\
MC stat. error (fitted) & 0.061 & 0.15 \\
Data stat. error (calculated) & 0.10 & 2.1 \\
\hline
\end{tabular}

Table 8.3: $\mathrm{MC}$ and Data statistical errors on $\mathcal{B}$ value.

\subsection{Systematic Errors from Analysis Method}

Shown in this section are the systematic errors which are derived from the analysis techniques used for this measurement of the branching fraction. Unless explicitly stated, the systematic error from each source is estimated by the difference between the measured $\mathcal{B}$ and the $\mathcal{B}$ value obtained in the systematic study. In each case, the on-peak data set is not changed in the study. Only the MC is affected. Thus, the statistical error from the data is exactly the same between main $\mathcal{B}$ measurement and the $\mathcal{B}$ value from the systematic study. Therefore, the statistical error is not taken into account in calculating the difference.

\subsection{1 $D^{*+} \mu^{-} \bar{\nu}_{\mu} \pi$ Background}

The generic $B^{0}$ and $B^{ \pm}$MC each contains three $D^{* *}$ decay modes and the nonresonant $B$ $\rightarrow D^{*+} \mu^{-} \bar{\nu}_{\mu} \pi$ decay modes which contribute to the $D^{*+} \mu^{-} \bar{\nu}_{\mu} \pi$ background, as described in Section 6.4. These eight modes are combined into a single histogram in order to fit for this background. The proportion of each particle in the generic $B \mathrm{MC}$ is determined 
by the world average of the measured branching fractions for the different decays ${ }^{[6]}$ or by theoretical estimates. However, given the current uncertainties on these measurements, the proportions of each decay in the background histogram is not well set.

In order to evaluate the systematic error in the $D^{* *}$ composition, single-mode MC samples for each $D^{* *}$ decay are generated. Each set can be individually used to represent to entire $D^{*+} \mu^{-} \bar{\nu}_{\mu} \pi$ background to see the systematic shift in the branching fraction. Unfortunately, when using only the Run 1 events, the size of each single-mode $D^{* *}$ sample is too small to make a meaningful fit. Instead, the generic $B \mathrm{MC}$ is used because the total number of $D^{* *}$ events is considerably larger. Each type of $D^{* *}$ decay is identified from MC truth. However, the statistics are still too small to use individual $D^{* *}$ and nonresonant $D^{*+} \mu^{-} \bar{\nu}_{\mu} \pi$ samples isolated from the generic MC. Instead, the studies are performed by removing the $D^{* *}$ and nonresonant samples one by one from the total set as listed in Table 8.4. The biggest shift in the branching fraction from removing the various $D^{* *}$ types is taken as the systematic error from the $D^{*+} \mu^{-} \bar{\nu}_{\mu} \pi$ background, which is $0.33 \%$ relative error.

\begin{tabular}{|l|c|c|c|}
\hline$D^{* *}$ Sample Removed & $\mathcal{B}\left(\bar{B}^{0} \rightarrow D^{*+} \mu^{-} \bar{\nu}_{\mu}\right)(\%)$ & $\delta \mathcal{B}(\%)$ & $\delta \mathcal{B} / \mathcal{B}(\%)$ \\
\hline $\bar{B}^{0} \rightarrow D_{1}^{+} \mu^{-} \bar{\nu}_{\mu}$ & 4.728 & 0.007 & 0.16 \\
$\bar{B}^{0} \rightarrow D_{1}^{*+} \mu^{-} \bar{\nu}_{\mu}$ & 4.719 & 0.002 & 0.03 \\
$\bar{B}^{0} \rightarrow D_{2}^{*+} \mu^{-} \bar{\nu}_{\mu}$ & 4.721 & 0.0002 & 0.005 \\
$\bar{B}^{0} \rightarrow D^{*+} \pi^{0} \mu^{-} \bar{\nu}_{\mu}$ & 4.711 & 0.010 & 0.20 \\
$B^{-} \rightarrow D_{1}^{0} \mu^{-} \bar{\nu}_{\mu}$ & 4.732 & 0.012 & 0.25 \\
$B^{-} \rightarrow D_{1}^{* 0} \mu^{-} \bar{\nu}_{\mu}$ & 4.736 & 0.015 & $\mathbf{0 . 3 3}$ \\
$B^{-} \rightarrow D_{2}^{* 0} \mu^{-} \bar{\nu}_{\mu}$ & 4.718 & 0.002 & 0.05 \\
$B^{-} \rightarrow D^{*+} \pi^{-} \mu^{-} \bar{\nu}_{\mu}$ & 4.708 & 0.012 & 0.26 \\
\hline
\end{tabular}

Table 8.4: Systematic effects of $D^{*+} \mu^{-} \bar{\nu}_{\mu} \pi$ background MC samples. Higher order $D^{* *}$ particles are not included in the generic $B$ MC.

\subsubsection{Muon Background Fractions}

The muon backgrounds, as described in Section 7.1.3, are combined together into one histogram. The scaling of this overall histogram is a parameter in the fit. The relative contribution of fake, correlated and uncorrelated muon backgrounds are taken from the generic $B \mathrm{MC}$ samples. In order to evaluate the effect of a different proportion of a particular sample, the relative sizes of each background sample is varied by $\pm 20 \%$ within the combined set, and the fit is redone. The largest difference will be taken as a conservative estimate of the systematic error due to the muon backgrounds. From Table 8.5, the systematic error for the muon backgrounds is $0.32 \%$. 


\begin{tabular}{|l|c|c|c|}
\hline Background shift & $\mathcal{B}(\%)$ & $\delta \mathcal{B}(\%)$ & $\delta \mathcal{B} / \mathcal{B}(\%)$ \\
\hline Correlated - 20\% & 4.710 & 0.010 & 0.22 \\
Correlated $+20 \%$ & 4.731 & 0.010 & 0.21 \\
Uncorrelated - 20\% & 4.716 & 0.004 & 0.089 \\
Uncorrelated + 20\% & 4.724 & 0.003 & 0.062 \\
Fake - 20\% & 4.736 & 0.015 & $\mathbf{0 . 3 2}$ \\
Fake $+20 \%$ & 4.707 & 0.013 & 0.29 \\
\hline
\end{tabular}

Table 8.5: Systematic effects of muon backgrounds.

\subsubsection{Truth Association}

The $\chi^{2}$ MC truth association procedure described in Section 6.1.1 is known to have approximately $1 \%$ probability of misassociation for low momentum particles. In order to examine the impact of this effect, the raw truth list is examined before any associations are made, and the number of $\bar{B}^{0} \rightarrow D^{*+} \mu^{-} \bar{\nu}_{\mu}\left(D^{0} \rightarrow K^{-} \pi^{+}\right)$decays per event is recorded. This is then compared to the truth associated categories for using $\bar{B}^{0} \rightarrow D^{*+} \mu^{-} \bar{\nu}_{\mu}$ $\left(D^{0} \rightarrow K^{-} \pi^{+}\right)$signal MC. All of the events identified as $\bar{B}^{0} \rightarrow D^{*+} \mu^{-} \bar{\nu}_{\mu}$ events do come from events with that decay. As expected, no events are being falsely identified as true signal events.

The main source of problem from the misassociated particles is the $D^{*}$ reconstruction. If the slow pion is not associated with its true track, a real $D^{*}$ can be labeled as a combinatoric $D^{*}$. This can cause a loss of efficiency if the event would otherwise be considered a true $\bar{B}^{0} \rightarrow D^{*+} \mu^{-} \bar{\nu}_{\mu}$ signal event. Of the events reconstructed from $\bar{B}^{0} \rightarrow$ $D^{*+} \mu^{-} \bar{\nu}_{\mu}\left(D^{0} \rightarrow K^{-} \pi^{+}\right)$signal MC, 32 are labeled as combinatoric. Of these 32, 30 come from events with a real $\bar{B}^{0} \rightarrow D^{*+} \mu^{-} \bar{\nu}_{\mu}\left(D^{0} \rightarrow K^{-} \pi^{+}\right)$decay. Compared to the 3755 events labeled as true signal decays, if the 30 combinatoric events should have been true signal, this corresponds to a loss of efficiency of $0.79 \%$. Since the efficiency factors directly into the final calculation of the branching fraction, this will be taken as the systematic error due to the MC truth associator.

\subsubsection{Reconstruction Efficiency}

The reconstruction efficiency is $11.03 \pm 0.18 \%$, as described in Section 7.2.1. This value enters the final $\mathcal{B}\left(\bar{B}^{0} \rightarrow D^{*+} \mu^{-} \bar{\nu}_{\mu}\right)$ calculation after the fit (Eq. (7.13)). Therefore, the relative error in this value is the relative error in the branching fraction from the efficiency. The systematic error from the reconstruction efficiency is $1.6 \%$.

\subsubsection{Continuum Background Representation}

Because only 4 events from the off-peak data pass all of the selection criteria, the statistics are too low for this sample to be usable in representing the continuum background. Therefore, as described in Section 6.6, the number of continuum background events is obtained from the $c \bar{c}$ MC simply by counting the number of events which pass all of the 
selection criteria. In order to confirm that the $\mathrm{MC}$ can be trusted to this extent, the number of events in the $c \bar{c}$ MC sample is compared to the off-peak sample. When the $c \bar{c} \mathrm{MC}$ is scaled to the equivalent luminosity of the off-peak sample, the corresponding number of events in the MC sample is 23.

This is significantly different from the 4 events observed in the off peak data. Therefore, a systematic error is measured for the continuum background. The $\cos \theta_{B, D^{*} \ell}$ fit is redone with the off-peak data used to determine the number of continuum background events. When the off-peak data is scaled to the equivalent luminosity of the on-peak sample, the number of continuum events is $32 \pm 16$. The shape of the $\cos \theta_{B, D^{*} \ell}$ distribution is still taken from the $c \bar{c}$ MC sample.

While there is only a single test here, the result is shown in Table 8.6 for visual consistency. The relative change in the branching fraction is $0.78 \%$, and this is taken as the systematic error in the continuum background representation.

\begin{tabular}{|c|c|c|c|}
\hline & $\mathcal{B}(\%)$ & $\delta \mathcal{B}(\%)$ & $\delta \mathcal{B} / \mathcal{B}(\%)$ \\
\hline Continuum from off-peak & 4.76 & 0.04 & 0.78 \\
\hline
\end{tabular}

Table 8.6: Systematic difference between off-peak data and $c \bar{c}$ MC for representing the number of continuum background events.

\subsection{BABAR Experiment Systematics}

This section describes systematic errors which are essentially the same throughout the collaboration. They are not specific to a particular analysis, although the specific value of certain systematic errors will depend on the analysis.

\subsubsection{PID Control Sample Statistics}

All MC is corrected using the PID efficiency tables for both data and MC, as described in Section 5.5. The efficiencies listed in the tables are limited in their precision by the size of the control samples in question. Mostly, these corrections have a very small effect on the shape of the template histograms in the fit, and the overall sizes are normalized, so the effect of these corrections on the fit results is very small. However, since the overall reconstruction efficiency is taken from the number of events in the the $D^{*+} \mu^{-} \bar{\nu}_{\mu}$ singlemode MC, the uncertainties in the PID corrections directly affect the reconstruction efficiency, and therefore the branching fraction.

The statistical error on the efficiencies has been evaluated by calculating an overall efficiency averaged over $(p, \theta, \phi)$ using generic $B \bar{B}$ and $B^{+} B^{-}$MC samples, and using the truth information to identify the particle species. The momentum and angle weighted efficiency is given by

$$
\epsilon=\frac{1}{\sum_{s} N_{s}} \sum_{n=1}^{m}\left(\sum_{s} N_{n ; s}\right) \omega(n),
$$


where $m$ is the total number of bins of $(p, \theta, \phi)$ in the efficiency table, $s=$ sample $(B \bar{B}$ or $\left.B^{+} B^{-}\right), N_{s}$ is the total number of events in sample $s, N_{n ; s}$ is the number of events from sample $\mathrm{s}$ that fall in bin $n$ of the efficiency table, and $\omega(n)$ is the efficiency in that bin. The uncertainty on the averaged efficiency is

$$
\delta \epsilon=\frac{1}{\sum_{s} N_{s}} \sqrt{\sum_{n=1}^{m}\left[\left(\sum_{s} N_{n ; s}\right)^{2} \delta \omega^{2}(n)+\left(\sum_{s} N_{n ; s}\right)\left(\omega^{2}(n)-\epsilon^{2}\right)\right]} .
$$

The first term in the sum over bins $(n=1$ to $m)$ corresponds to the uncertainty due to the uncorrelated statistical errors $\delta \omega(n)$ on the efficiency calculated from the control samples. The second term corresponds to the statistical uncertainty due to the MC sample used for the angle and momentum weighting. ${ }^{[61]}$

As Table 8.7 shows, the muon Very-Tight selector from the $\mu^{+} \mu^{-} \gamma$ control sample has the largest statistical uncertainty at $0.61 \%$ relative. This error will be considered an error in the muon identification.

\begin{tabular}{|l|c|c|c|}
\hline Selector & Control Sample & Efficiency (\%) & $\delta \epsilon / \epsilon(\%)$ \\
\hline Muon (Very Tight) & $e^{+} e^{-} \rightarrow \mu^{+} \mu^{-} \gamma$ & $67.54 \pm 0.41$ & $\mathbf{0 . 6 1}$ \\
Muon (Very Tight) & $e^{+} e^{-} \rightarrow e^{+} e^{-} \mu^{+} \mu^{-}$ & $68.08 \pm 0.12$ & 0.18 \\
Muon (Very Tight) & $\mathrm{MC}$ Truth & $70.958 \pm 0.055$ & 0.078 \\
\hline Kaon (Not-a-Pion) & (Data) $D^{0} \rightarrow K^{-} \pi^{+}$ & $97.519 \pm 0.095$ & 0.097 \\
Kaon (Not-a-Pion) & (MC) $D^{0} \rightarrow K^{-} \pi^{+}$ & $97.97 \pm 0.10$ & 0.10 \\
\hline
\end{tabular}

Table 8.7: Average efficiencies for different control samples.

\subsubsection{PID Corrections}

There are two control samples used to characterize the muons, as described in Section 4.2.4. The results in this thesis use the $\mu^{+} \mu^{-} \gamma$ control sample as the standard because of the work that I did in order to generate this control sample for BABAR. In order to study the systematic effect on the choice of control sample for the muons, the $\mu^{+} \mu^{-} \gamma$ based efficiency table is replaced with the $е e \mu \mu$ based table. Table 8.8 shows the $0.66 \%$ relative shift in the $\mathcal{B}$ value which is only as large as the statistical uncertainty of the $\mu^{+} \mu^{-} \gamma$ control sample $-0.61 \%$ from Table 8.7 - so the systematic uncertainty based on the choice of control sample cannot be well determined, but it would not be a significant component.

\begin{tabular}{|l|c|c|c|}
\hline Control sample & $\mathcal{B}(\%)$ & $\delta \mathcal{B}(\%)$ & $\delta \mathcal{B} / \mathcal{B}(\%)$ \\
\hline$е е \mu \mu$ & 4.75 & 0.03 & 0.66 \\
\hline
\end{tabular}

Table 8.8: Systematic effect of muon control sample. 


\subsubsection{Tracking Efficiency}

The corrections applied to the MC to account for differences in the tracking efficiency are described in Section 5.5.1. For each track corrected, there is an associated systematic error of $0.8 \%$. Adding the correlated errors linearly for the three corrected tracks in the GoodTracksLoose list produces a total systematic error of $2.4 \%$. The systematic error determined for the slow pion is $1.3 \%$, as described in Section 5.5.2.

\subsubsection{Beam Energy Data/MC Disagreement}

The $\mathrm{MC}$ is modeled based on a constant beam energy of $10.58 \mathrm{GeV}$. However, the exact energy fluctuates slightly due to changes in accelerator tuning and conditions. The correction to the beam energy is described in Section 5.5.4. This correction causes the $\mathcal{B}$ to change by 0.071 . A systematic error of $1 / 2$ of this shift has been estimated for this correction, or $0.75 \%$ relative.

\subsubsection{HQET Parameter Dependence}

The $\bar{B}^{0} \rightarrow D^{*+} \ell^{-} \bar{\nu}_{\ell}$ decay is modeled in the MC using HQET. In order to evaluate the dependence on the HQET model parameters, $R_{1}, R_{2}$, and $\rho^{2}$ as described in Section 2.4, special $\bar{B}^{0} \rightarrow D^{*+} \ell^{-} \bar{\nu}_{\ell}$ single-mode Monte Carlo samples have been generated. In each sample, one of the three parameters has been varied by one standard deviation from the reference MC value, whose inputs come from the measured CLEO values in linear HQET. ${ }^{[66]}$ This provides six samples to study.

To study the effect of each sample, the $D^{*+} \mu^{-} \bar{\nu}_{\mu}$ single-mode MC sample is replaced with the HQET sample in the main fit, and a new count of the $D^{*+} \mu^{-} \bar{\nu}_{\mu}$ decays is obtained. The reconstruction efficiency is also calculated from the HQET sample in order to obtain a new value for the branching fraction. Due to the limited size of these MC samples, the statistical error in the result is a problem. Each sample represents a combination of the total data from 1999-2002, of which approximately one-third represents the 1999/2000 run period. Unfortunately, the statistical error based on one-third of each set is $7-8 \%$, which is much higher than the systematic contribution from the HQET parameters, so the full data set is needed to reduce the statistical uncertainty. However, if the full data set is used, further systematic effects could be introduced by the gradual loss in muon efficiency in the IFR. In order to reduce this effect, the difference between the branching fractions for the high $(+\sigma)$ and low $(-\sigma)$ settings of each parameter are taken. One-half of this difference is taken as the systematic error, provided it is larger than the statistical error.

As can be seen in Table 8.9, only the $\rho^{2}$ parameter provides a large enough shift. It is larger than, but still close to the size of the statistical error. The systematic error can be isolated by subtracting the statistical error in quadrature from the difference:

$$
\text { Syst. }=\sqrt{\Delta^{2}-\text { Stat. }^{2}}
$$




\begin{tabular}{|l|c|c|}
\hline Parameter shift & $\mathcal{B}(\%)$ & $\Delta(\%)$ \\
\hline \hline$R_{1}+\sigma$ & $4.934+/-0.22$ & \\
$R_{1}-\sigma$ & $5.129+/-0.23$ & $0.098+/-0.16$ \\
\hline$R_{2}+\sigma$ & $4.869+/-0.21$ & \\
$R_{2}-\sigma$ & $5.023+/-0.22$ & $0.077+/-0.15$ \\
\hline$\rho^{2}+\sigma$ & $5.168+/-0.22$ & \\
$\rho^{2}-\sigma$ & $4.801+/-0.21$ & $0.184+/-0.15$ \\
\hline
\end{tabular}

Table 8.9: $\mathcal{B}$ shifts with HQET parameters. $\Delta=\left|\mathcal{B}_{+\sigma}-\mathcal{B}_{-\sigma}\right| / 2$ for each parameter.

where $\Delta=\left(\mathcal{B}_{+\sigma}-\mathcal{B}_{-\sigma}\right) / 2$. This method has been used by other published analyses in BABAR. ${ }^{[10]}$ This yields a systematic error of 0.102 or a $2.2 \%$ error relative to the $\bar{B}^{0} \rightarrow D^{*+} \mu^{-} \bar{\nu}_{\mu}$ branching fraction.

\subsubsection{Number of $\Upsilon(4 S)$ Produced}

The number of $\Upsilon(4 S)$ events produced in Run 1 is $(2.232 \pm 0.025) \times 10^{7}$, as described in Section 3.2. This value enters the final $\mathcal{B}\left(\bar{B}^{0} \rightarrow D^{*+} \mu^{-} \bar{\nu}_{\mu}\right)$ calculation. The systematic error from the $\Upsilon(4 S)$ counting procedure ${ }^{[32]}$ is $1.1 \%$.

\subsection{Systematics from Externally Measured Branching Fractions}

The branching fractions for the other decays involved in the reconstruction have been measured by other experiments. The values used in this analysis are taken from the 2002 Particle Data Book. ${ }^{[6]}$ The systematic error quoted is the total error on the published value. Since these branching fractions are used in the final $\mathcal{B}\left(\bar{B}^{0} \rightarrow D^{*+} \mu^{-} \bar{\nu}_{\mu}\right)$ calculation, the systematic errors from these values are the relative errors listed in the published values.

\begin{tabular}{|l|c|c|}
\hline Parameter & Value (\%) & Error (\%) \\
\hline $\mathcal{B}\left(\Upsilon(4 S) \rightarrow B^{0} \bar{B}^{0}\right)^{[64]}$ & $48.7 \pm 1.3$ & 2.7 \\
$\mathcal{B}\left(D^{*+} \rightarrow D^{0} \pi^{+}\right)^{[6]}$ & $67.7 \pm 0.5$ & 0.7 \\
$\mathcal{B}\left(D^{0} \rightarrow K^{-} \pi^{+}\right)^{[6]}$ & $3.80 \pm 0.09$ & 2.4 \\
\hline
\end{tabular}

Table 8.10: Values entering final $\mathcal{B}$ calculation

\subsection{Systematic Error Summary}

Table 8.11 is a summary of the various systematic errors in the $\mathcal{B}\left(\bar{B}^{0} \rightarrow D^{*+} \mu^{-} \bar{\nu}_{\mu}\right)$ measurement. All values are percentage errors. The total is the quadrature sum of all the systematic errors. 
Four sources dominate the systematic errors in this analysis: tracking efficiency, the HQET parameters, and the branching fractions, $\mathcal{B}\left(\Upsilon(4 S) \rightarrow B^{0} \bar{B}^{0}\right)$ and $\mathcal{B}\left(D^{0} \rightarrow K^{-}\right.$ $\left.\pi^{+}\right)$. The two branching fractions are both measured at other experiments. The BABAR MC simulation of the $\bar{B}^{0} \rightarrow D^{*+} \mu^{-} \bar{\nu}_{\mu}$ decay is based on the HQET parameters. The systematic error in the tracking efficiency corrections applies to all BABAR analyses. Thus, improvements to this analysis would not improve these systematic errors.

Other analyses in BABAR are studying the HQET parameters and the $\mathcal{B}(\Upsilon(4 S) \rightarrow$ $\left.B^{0} \bar{B}^{0}\right)$. So results from these studies could lead to some improvement in the precision of this $\mathcal{B}\left(\bar{B}^{0} \rightarrow D^{*+} \mu^{-} \bar{\nu}_{\mu}\right)$ measurement, but there would still be the large error in the tracking efficiency corrections.

\begin{tabular}{|c|c|c|}
\hline Source & $\delta \mathcal{B} / \mathcal{B}(\%)$ & $\delta \mathcal{B} / \mathcal{B}(\%)$ \\
\hline \multicolumn{3}{|l|}{ Analysis Based } \\
\hline$D^{*+} \mu^{-} \bar{\nu}_{\mu} \pi$ background & 0.33 & \\
\hline Muon backgrounds & 0.32 & \\
\hline MC truth association & 0.79 & \\
\hline Reconstruction efficiency & 1.6 & \\
\hline Continuum background representation & 0.78 & \\
\hline \multicolumn{2}{|l|}{ Subtotal } & 2.0 \\
\hline \multicolumn{3}{|l|}{ BABAR Experiment Based } \\
\hline Tracking efficiency & 2.4 & \\
\hline Slow pion efficiency & 1.3 & \\
\hline Muon identification & 0.61 & \\
\hline Beam energy data/MC disagreement & 0.75 & \\
\hline HQET parameter dependence & 2.2 & \\
\hline$B$ counting & 1.1 & \\
\hline \multicolumn{2}{|l|}{ Subtotal } & 3.8 \\
\hline \multicolumn{3}{|l|}{ Other Branching Fractions } \\
\hline $\mathcal{B}\left(\Upsilon(4 S) \rightarrow B^{0} \bar{B}^{0}\right)$ & 2.7 & \\
\hline $\mathcal{B}\left(D^{*+} \rightarrow D^{0} \pi^{+}\right)$ & 0.7 & \\
\hline $\mathcal{B}\left(D^{0} \rightarrow K^{-} \pi^{+}\right)$ & 2.4 & \\
\hline \multicolumn{2}{|l|}{ Subtotal } & 3.7 \\
\hline \multicolumn{2}{|l|}{ Total } & 5.7 \\
\hline
\end{tabular}

Table 8.11: Summary of systematic errors. 


\section{Chapter 9}

\section{Conclusion}

From the 22.3 million $\Upsilon(4 S)$ events recorded by BABAR during 1999/2000, $2911 \pm 73$ $\bar{B}^{0} \rightarrow D^{*+} \mu^{-} \bar{\nu}_{\mu}$ events are reconstructed. From this, the value of the $\bar{B}^{0} \rightarrow D^{*+} \mu^{-} \bar{\nu}_{\mu}$ branching fraction has been determined to be

$$
\begin{aligned}
\mathcal{B}\left(\bar{B}^{0} \rightarrow D^{*+} \mu^{-} \bar{\nu}_{\mu}\right)=4.72 & \pm 0.10 \text { (data stat.) } \pm 0.06 \text { (MC stat.) } \\
& \pm 0.20 \text { (syst.) } \pm 0.17 \text { (other } \mathcal{B}) \%
\end{aligned}
$$

The statistical error in the $\mathcal{B}$ measurement is almost half of the total systematic error from the experiment. Therefore, higher statistics would not improve the overall result significantly. The largest systematic error in the $\mathcal{B}$ measurement comes from the externally measured branching fraction for $\Upsilon(4 S) \rightarrow B^{0} \bar{B}^{0}$, as shown in Table 8.11. The largest systematic error from the experiment is the particle tracking efficiency. This is not a function of this analysis, but common to the entire BABAR experiment. All $\mathrm{MC}$, regardless of how many events are generated, would require this tracking efficiency correction and the corresponding systematic error. Thus, the precision would not be improved with more data.

The BABAR $\left|V_{c b}\right|$ analysis group recently presented a preliminary conference result for this branching fraction of $4.68 \pm 0.03$ (stat.) \pm 0.29 (syst.). ${ }^{[10]}$ Comparing to this preliminary result shows that the two values are in excellent agreement, even within the statistical errors. Thus, this analysis serves as a good cross check to show the robustness of the BABAR analysis for this branching fraction. The $\left|V_{c b}\right|$ analysis uses $79.1 \mathrm{fb}^{-1}$ of onpeak data and a comparatively larger sample of MC simulated data. It also reconstructs several more $D^{0}$ decay modes than just $D^{0} \rightarrow K^{-} \pi^{+}$. While the statistical errors are considerably smaller, the systematic errors remain essentially the same compared to this analysis. Therefore, the extra data has not improved the precision of this branching fraction measurement. However, the higher statistics provide a much better precision for the measurement of $\left|V_{c b}\right|$ presented in the same paper. ${ }^{[10]}$

Figure 9.1 shows a comparison of this measurement with other experiments that make up the current world average according to the 2003 Particle Data Group review. ${ }^{[7]}$ There is very good agreement with the other experiments and the 2002 world average. However, the new CLEO result is higher than several previous results and causes the 2003 world average to rise compared to the 2002 average, but the difference is not statistically significant.

Therefore, this analysis provides a good measurement of the $\bar{B}^{0} \rightarrow D^{*+} \mu^{-} \bar{\nu}_{\mu}$ branching fraction. The value is in good agreement with the main $B A B A R$ measurement from 


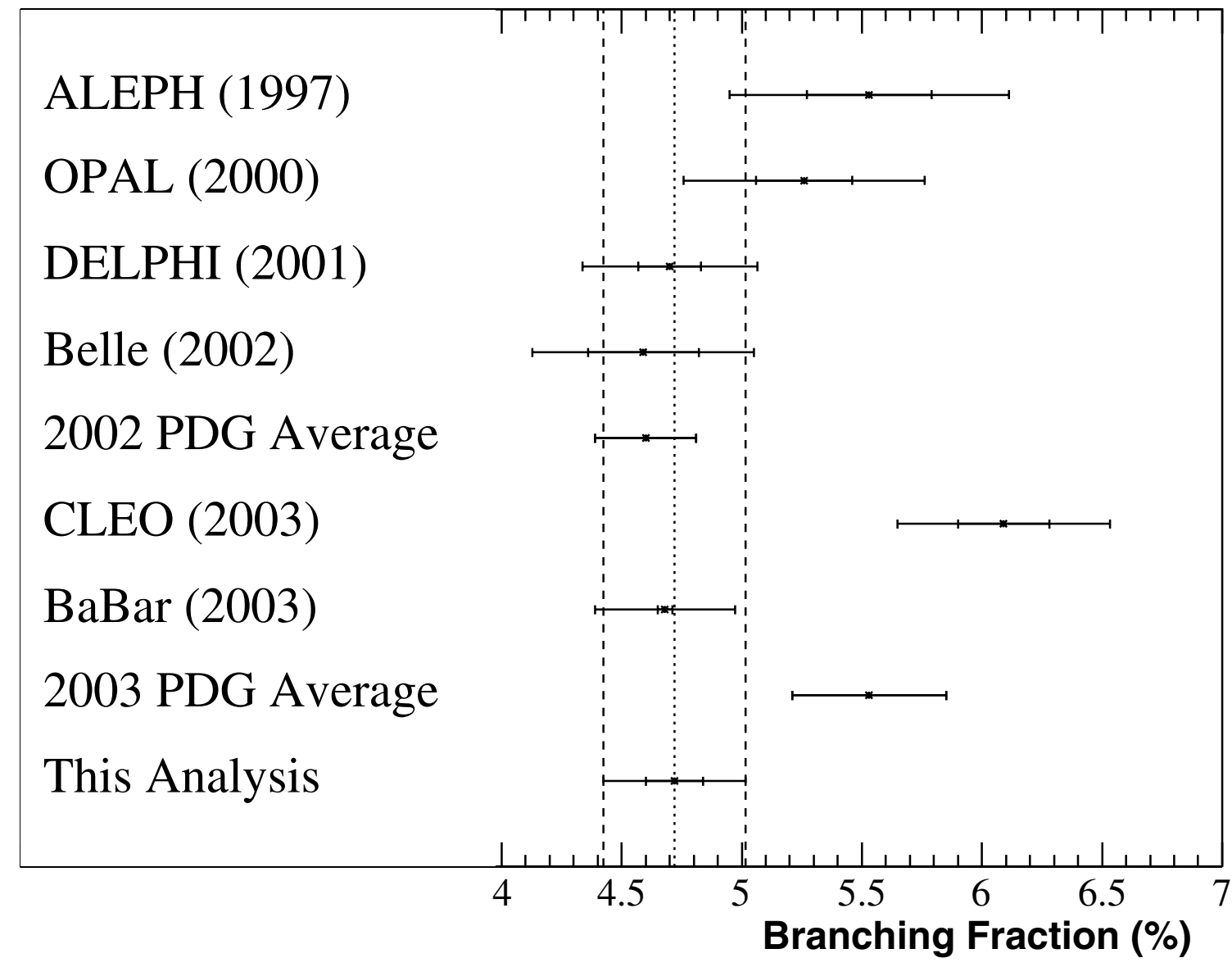

Figure 9.1: Comparison to $\mathcal{B}\left(\bar{B}^{0} \rightarrow D^{*+} \mu^{-} \bar{\nu}_{\mu}\right)$ measurements from BABAR and other experiments. This analysis shows very good agreement to the other results, with the possible exception of the CLEO measurement. ${ }^{[6,7,10,67-71]}$

the $\left|V_{c b}\right|$ analysis and with the results from experiments around the world. The precision of this result is as good as the main BABAR measurement and better than the currently published results. 


\section{Bibliography}

[1] N. Cabibbo, "Unitary Symmetry and Leptonic Decays," Phys. Rev. Lett. 10, 531 (1963).

[2] M. Kobayashi and T. Maskawa, "CP Violation in the Renormalizable Theory of Weak Interaction," Prog. Theor. Phys. 49, 652 (1973).

[3] S. L. Glashow, "Partial Symmetries of Weak Interactions," Nucl. Phys. 22, 579 (1961).

[4] S. Weinberg, "A Model of Leptons," Phys. Rev. Lett. 19, 1264 (1967).

[5] A. Salam and J. C. Ward, "Electromagnetic and Weak Interactions," Phys. Lett. 13, 168 (1964).

[6] K. Hagiwara et al. [Particle Data Group Collaboration], "Review of Particle Physics," Phys. Rev. D 66, 010001 (2002).

[7] K. Hagiwara et al. [Particle Data Group Collaboration], "Review of Particle Physics," Phys. Rev. D 66, 010001 (2002). and 2003 off-year partial update for the 2004 edition available on the PDG WWW pages (URL: http://pdg.lbl.gov/).

[8] M. E. Luke, "Effects of Subleading Operators in the Heavy Quark Effective Theory," Phys. Lett. B 252, 447 (1990).

[9] M. Neubert and V. Rieckert, "New Approach To The Universal Form-Factors In Decays Of Heavy Mesons," Nucl. Phys. B 382, 97 (1992).

[10] B. Aubert et al. [BABAR Collaboration], "Measurement of $\left|V_{c b}\right|$ using $\bar{B}^{0} \rightarrow$ $D^{*+} \ell^{-} \bar{\nu}_{\ell}$ decays," arXiv:hep-ex/0308027.

[11] R. Dubitzky. [BABAR Collaboration], "Measurement of the CKM Matrix Element $\left|V_{c b}\right|$ with $B^{0} \rightarrow D^{*-} e^{+} \nu_{e}$ Decays," BABAR Analysis Document 527 v.05 (2003).

[12] E. Noether, "Invariante Variationsprobleme,"

Nach. v. d. Ges. d. Wiss. zu Göttingen (1918) 235.

[13] P. W. Higgs, "Broken Symmetries, Massless Particles and Gauge Fields," Phys. Lett. 12, 132 (1964).

[14] F. Gross, "Relativistic Quantum Mechanics and Field theory," John Wiley \& Sons, Inc. (1993). 
[15] C. S. Wu, E. Ambler, R. W. Hayward, D. D. Hoppes and R. P. Hudson, "Experimental Test of Parity Conservation in Beta Decay,"

Phys. Rev. 105, 1413 (1957).

[16] J. H. Christenson, J. W. Cronin, V. L. Fitch and R. Turlay, "Evidence For the $2 \pi$ Decay of the $K_{2}^{0}$ Meson," Phys. Rev. Lett. 13, 138 (1964).

[17] B. Aubert et al. [BABAR Collaboration], "Observation of CP violation in the $B^{0}$ meson system,"

Phys. Rev. Lett. 87, 091801 (2001)

[18] K. Abe et al. [Belle Collaboration], "Observation of large CP violation in the neutral $B$ meson system,"

Phys. Rev. Lett. 87, 091802 (2001)

[19] L. Wolfenstein, "Parametrization of the Kobayashi-Maskawa Matrix," Phys. Rev. Lett. 51, 1945 (1983).

[20] N. Isgur and M. B. Wise, "Weak Decays of Heavy Mesons in the Static Quark Approximation," Phys. Lett. B 232, 113 (1989).

[21] M. A. Shifman and M. B. Voloshin, "On Annihilation of Mesons Built From Heavy and Light Quark and $\bar{B}^{0} \leftrightarrow B^{0}$ Oscillations," Sov. J. Nucl. Phys. 45, 292 (1987)

[22] M. A. Shifman and M. B. Voloshin, "On Production of $D$ and $D^{*}$ Mesons in $B$ Meson Decays," Sov. J. Nucl. Phys. 47, 511 (1988)

[23] N. Isgur and M. B. Wise, "Weak Transition Form-Factors Between Heavy Mesons," Phys. Lett. B 237, 527 (1990).

[24] M. Wirbel, B. Stech and M. Bauer, "Exclusive Semileptonic Decays of Heavy Mesons," Z. Phys. C 29, 637 (1985).

[25] M. Bauer, B. Stech and M. Wirbel, "Exclusive Nonleptonic Decays of $D, D_{s}^{+}$, and B Mesons," Z. Phys. C 34, 103 (1987).

[26] M. Neubert, "Model Independent Extraction of $\left|V_{c b}\right|$ From Semileptonic Decays," Phys. Lett. B 264, 455 (1991).

[27] M. Neubert, "Heavy quark symmetry," Phys. Rept. 245, 259 (1994)

[28] D. Boutigny et al. [BABAR Collaboration], "BABAR technical design report," SLAC-R-0457

[29] B. Aubert et al. [BABAR Collaboration], "The BABAR detector," Nucl. Instrum. Meth. A 479, 1 (2002) 
[30] J. Seeman et al., "Status report on PEP-II performance," Presented at 7th European Particle Accelerator Conference (EPAC 2000), Vienna, Austria, 26-30 Jun 2000. SLAC-PUB-8629

[31] M. Sullivan, "B-factory interaction region design," Talk given at 17th IEEE Particle Accelerator Conference (PAC 97): Accelerator Science, Technology and Applications, Vancouver, Canada, 12-16 May 1997. SLAC-PUB-7563

[32] C. Hearty, [BABAR Collaboration], "Measurement of the number of $\Upsilon(4 S)$ mesons produced in run 1 (B counting)," BABAR Analysis Document 134 v.01 (2001).

[33] B. Aubert et al. [BABAR Collaboration], "Study of inclusive production of charmonium mesons in B decay," Phys. Rev. D 67, 032002 (2003)

[34] D. Lange [BABAR Collaboration], "Muon Efficiency vs Run Number," BABAR internal web page. (2002), http://www.slac.stanford.edu/BFROOT/www/ Detector/IFR/lange/IfrBarrelUpgrade/bpcPlots/mueffVsrun_barl.eps

[35] F. Forti et al. [BABAR Collaboration], "Report of the IFR Barrel Replacement Review Committee," BABAR Analysis Document 652 v.01 (2003).

[36] P. Billoir, "Track Fitting With Multiple Scattering: A New Method," Nucl. Instrum. Meth. A 225, 352 (1984).

[37] C. Roat. "Candidate Lists and Maps in the Micro Database." BABAR internal web page. (2001), http://www.slac.stanford.edu/BFROOT/www/ doc/workbook/nanomicro/v8.8/Micro/CandLists.html

[38] S. Sewerynek, "A Monte Carlo Study of the Momentum Dependence on the Results of Tracking Unknown Particle Species in the BaBar Detector." M.Sc Thesis, UBC, 1999.

[39] F. Fabozzi et al. [BABAR Collaboration], "Muon Identification in the BABAR Experiment," BABAR Analysis Document 60 v.01 (2000).

[40] G. Mancinelli, S. Spanier. [BABAR Collaboration], "Kaon Selection at the BABAR Experiment," BABAR Analysis Document 116 v.01 (2001).

[41] U. Langenegger et al. [BABAR Collaboration], "Cut-based Electron Identification," BABAR Analysis Document 90 v.05 (2001).

[42] D. Thiessen. [BABAR Collaboration], "A New $\mu \mu \gamma$ Control Sample," BABAR Analysis Document 268 v.01 (2001).

[43] B. Barish et al. [CLEO Collaboration], "Measurement of the $\bar{B}^{0} \rightarrow D^{*+} \ell^{-} \bar{\nu}_{\ell}$ branching fractions and $\left|V_{c b}\right|$, , Phys. Rev. D 51, 1014 (1995) 
[44] C. Borean et al. [BABAR Collaboration], "Measurement of the $\bar{B}^{0} \rightarrow D^{*+} \ell^{-} \bar{\nu}_{\ell}$ Branching Ratio using the 2001 Data Sample," BABAR Analysis Document 457 v.06 (2003).

[45] S. Agostinelli et al. [GEANT4 Collaboration], "GEANT4: A simulation toolkit," Nucl. Instrum. Meth. A 506, 250 (2003).

[46] D. E. Groom et al. [Particle Data Group Collaboration], "Review of Particle Physics," Eur. Phys. J. C 15, 1 (2000).

[47] M. Carpinelli et al. [BABAR Collaboration], "The BABAR Vertexing," BABAR Analysis Document 102 v.06 (2001).

[48] M. Bona et al. [BABAR Collaboration], "Report of the Tracking Efficiency Task Force" BABAR Analysis Document 324 v.02 (2002).

[49] O. Long [BABAR Collaboration], "Measurement of the slow pion relative efficiency using helicity distributions." BABAR Analysis Document 54 v.07 (2001).

[50] D. Mascher et al. [BABAR Collaboration], "Determination of the Mass Difference $m\left(B^{0}\right)-m\left(B^{+}\right)$using $J / \psi K^{+}$and $J / \psi K^{* 0}$ Decays from $82.2 \Upsilon(4 S)$ Events" BABAR Analysis Document 566 v.08 (2003).

[51] M. Gill [BABAR Collaboration], "DstarLNuUser Variable List," BABAR internal web page. (2002), http://www.slac.stanford.edu/ msgill/Dstar/dlnu_varlist.html

[52] D. Buskulic et al. [ALEPH Collaboration], "Production of orbitally excited charm mesons in semileptonic B decays," Z. Phys. C 73, 601 (1997).

[53] J. L. Goity and W. Roberts, "Soft Pion Emission in Semileptonic B Meson Decays," Phys. Rev. D 51, 3459 (1995)

[54] D. Scora and N. Isgur, "Semileptonic meson decays in the quark model: An update," Phys. Rev. D 52, 2783 (1995)

[55] P. Avery et al. [CLEO Collaboration], "Production and decay of $D_{1}^{0}(2420)$ and $D_{2}^{* 0}$ (2460)," Phys. Lett. B 331, 236 (1994) [Erratum-ibid. B 342, 453 (1995)]

[56] T. Bergfeld et al. [CLEO Collaboration], "Observation of $D_{1}^{+}(2420)$ and $D_{2}^{*+}$ (2460)," Phys. Lett. B 340, 194 (1994).

[57] P. Abreu et al. [DELPHI Collaboration], "First evidence for a charm radial excitation, $D^{*}$, ," Phys. Lett. B 426, 231 (1998).

[58] R. Brun and F. Rademakers, "Root: An Object Oriented Data Analysis Framework," Nucl. Instrum. Meth. A 389, 81 (1997). 
[59] W. Verkerke and D. Kirkby, "The RooFit Toolkit for Data Modeling," (2003), http://roofit.sourceforge.net/

[60] F. James and M. Roos, "Minuit' A System For Function Minimization and Analysis of the Parameter Errors and Correlations," Comput. Phys. Commun. 10, 343 (1975).

[61] C.H. Cheng et al. [BABAR Collaboration], "Semileptonic B Sample for Lifetime and Mixing Analyses", BABAR Analysis Document 147 v.06 (2002).

[62] S. Baker and R.D. Cousins, "Clarification of the Use of Chi Square and Likelihood Functions in Fits To Histograms," Nucl. Instrum. Meth. A 221, 437 (1984).

[63] R. J. Barlow and C. Beeston, "Fitting Using Finite Monte Carlo Samples," Comput. Phys. Commun. 77, 219 (1993).

[64] L. Gibbons and K. Honschied, "Production and Decay of b-Flavored Hadrons," Errata to 2002 Particle Data Book ${ }^{[6]}$ (2002), http://pdg.lbl.gov/2002/bmesondecay_s841.ps

[65] B. Aubert et al. [BABAR Collaboration], "Measurement of branching fractions for exclusive $B$ decays to charmonium final states," Phys. Rev. D 65, 032001 (2002)

[66] J. E. Duboscq et al. [CLEO Collaboration], "Measurement of the form-factors for $\bar{B}^{0} \rightarrow D^{*+} \ell^{-} \bar{\nu}_{\ell}, "$ Phys. Rev. Lett. 76, 3898 (1996).

[67] N. E. Adam et al. [CLEO Collaboration], "Determination of the $\bar{B}^{0} \rightarrow D^{*+} \ell^{-} \bar{\nu}_{\ell}$ decay width and $\left|V_{c b}\right|$. ((B))," Phys. Rev. D 67, 032001 (2003)

[68] K. Abe et al. [BELLE Collaboration], "Determination of $\left|V_{c b}\right|$ using the semileptonic decay $\bar{B}^{0} \rightarrow D^{*+} e^{-} \bar{\nu}_{e}, "$ Phys. Lett. B 526, 247 (2002)

[69] P. Abreu et al. [DELPHI Collaboration], "Measurement of $\left|V_{c b}\right|$ from the decay process $\bar{B}^{0} \rightarrow D^{*+} \ell^{-} \bar{\nu}_{\ell}, "$ Phys. Lett. B 510, 55 (2001)

[70] G. Abbiendi et al. [OPAL Collaboration], "Measurement of $\left|V_{c b}\right|$ using $\bar{B}^{0} \rightarrow$ $D^{*+} \ell^{-} \bar{\nu}_{\ell}$ decays," Phys. Lett. B 482, 15 (2000)

[71] D. Buskulic et al. [ALEPH Collaboration], "Measurements of $\left|V_{c b}\right|$, form factors and branching fractions in the decays $\bar{B}^{0} \rightarrow D^{*+} \ell^{-} \bar{\nu}_{\ell}$ and $\bar{B}^{0} \rightarrow D^{+} \ell^{-} \bar{\nu}_{\ell}, "$ Phys. Lett. B 395, 373 (1997). 


\section{Glossary}

BG Background

CM Centre-of-mass

CKM Cabibbo-Kobayashi-Maskawa

DCH Drift chamber

$\mathrm{d} E / \mathrm{d} x$ Ionization energy per unit distance in the DCH or SVT.

DIRC Detector of internally reflected Cherenkov

$\delta m$ Mass difference $m_{D^{*}}-m_{D^{0}}$

DOCA Distance of closest approach

EMC Electromagnetic calorimeter

HER High-energy (electron) ring

HQET Heavy quark effective theory

IFR Instrumented flux return

IP Interaction point

IR Interaction region

L1 Level 1 trigger

L3 Level 3 trigger

LER Low-energy (positron) ring

MC Monte Carlo simulated data

MIP Minimum ionizing particle

OPR Online prompt reconstruction

PDF Probability density function

PID Particle identification 
110

PMT Photomultiplier tubes

QCD Quantum chromodynamics

RPC Resistive plate chamber

SLAC Stanford Linear Accelerator Center

SVT Silicon vertex tracker

TIR Total internal reflection 


\section{Appendix A}

\section{The $\mu^{+} \mu^{-} \gamma$ Control Sample}

This appendix is a reproduction of BABAR Analysis Document \#268 ${ }^{[42]}$ which I wrote for the collaboration. It is the report on the creation and analysis of a $\mu \mu \gamma$ control sample which I performed in service to the central physics effort of BABAR.

Btamumugamma2Sample is a module in the BetaPidCalib ${ }^{\mathrm{a}}$ that selects muon tracks for a high purity control sample based on the reaction $e^{+} e^{-} \rightarrow \mu^{+} \mu^{-} \gamma$. It was generated in order to provide a cross-check on the $e e \mu \mu$ control sample which is used to produce the muon ID efficiency tables currently used by BaBar. Analysis of $\mu$ ID efficiency differences between the two samples shows a possible contamination in the ee $\mu \mu$ sample at low momentum in the forward end-cap.

\section{A.1 Introduction}

Muons from the reaction $e^{+} e^{-} \rightarrow \mu^{+} \mu^{-} \gamma$, subsequently referred to as $\mu \mu \gamma$, provide a good control sample for measuring muon ID efficiency. The final state $\gamma$ allows muon tracks to be well distributed over the full solid angle of the detector with a much lower $p-\theta$ correlation than non-radiative di-muons. While the majority of muon tracks have a high momentum, the sample also extends down to below $500 \mathrm{MeV} / c$.

This sample can be used to calculate the efficiencies of our current muon ID algorithms. The current muon efficiency tables are based on a control sample using the two-photon $e^{+} e^{-} \rightarrow e^{+} e^{-} \mu^{+} \mu^{-}$reaction $(e e \mu \mu)$. The $\mu \mu \gamma$ sample provides an independent source of pure muons and allows a cross check of the efficiency tables. A $\mu \mu \gamma$ control sample was generated previously, but the efficiencies calculated from that sample were systematically lower than those from the ee $\mu \mu$ sample. ${ }^{[39]}$ This control sample has been generated completely independently of the first sample and uses different selection techniques.

\section{A.2 Track Selection}

The runs used in this analysis were the full Run 1 data set. The muon tracks were selected from two sources in the database. The majority of the events pass the BGFTwoProng ${ }^{b}$ filter, which is designed to select muon pair events and suppress Bhabhas. Events are also

\footnotetext{
${ }^{a}$ BetaPidCalib is a module which runs during OPR processing which package that tags certain events of interest to the particle ID group.

${ }^{\mathrm{b}}$ BGFTwoProng is a list analogous to the tracking lists such as GoodTracksLoose.
} 
selected from the DigiL3Prescale ${ }^{\mathrm{c}}$ sample with the requirement that there are exactly 2 oppositely-charged tracks.

The pair of tracks is then combined with each photon in the event. Photons are taken from the GoodPhotonLoose list, and the photon selected is the one which brings the total invariant mass of the 2 tracks + photon closest to the event mass. This is not necessarily the highest energy photon in the event. A cut is applied requiring that the energy of the photon be at least $1.5 \mathrm{GeV}$. This eliminates low energy photons which could be noise, and it significantly reduces the number of events in which the momenta of both tracks are $>4 \mathrm{GeV} / \mathrm{c}$, while preserving events with lower momentum tracks. Since the $\mu$ ID tables only extend up to $4 \mathrm{GeV} / \mathrm{c}$, it significantly reduces the amount of unnecessary muon tracks in the sample while still providing the possibility of extending the tables. A further cut is applied requiring that the energy of any other photon in the event be less than $50 \mathrm{MeV}$.

A kinematic fit is then applied to the 2 track + photon vertex. The constraints applied to the fit are:

- geometric constraint with the vertex required to be within the beam spot.

- $\mu \mu \gamma$ invariant mass constrained to the event mass.

- $\mu \mu \gamma$ momentum constrained to the total beam momentum.

The chi-squared probability of the fit was required to be $>0.01$. This eliminates virtually all the $\tau$ events because of the missing momentum carried by the neutrino in the $\tau$ decay. A further cut was applied on the event requiring that the calorimeter centroid of the photon candidate to be at least $30 \mathrm{~cm}$ apart from each muon centroid to avoid overlap.

A track from an event passing these cuts is added to the list of unbiased muons if the oppositely charged track passes the standard BaBar loose muon selector. ${ }^{[39]}$ No further cuts are applied to the tracks on this list. They are unbiased with respect to the $\mu$ ID algorithms and are used to calculate the efficiencies of these algorithms.

\section{A.3 Purity}

The two major sources of contamination are other radiative di-leptons. However, contamination by ee $\gamma$ and $\tau \tau \gamma$ events have been cut to a very low level, resulting in a sample of high purity. Samples of eer, $\mu \mu \gamma$, and $\tau \tau$ events from the SP3 Monte Carlo were studied to determine the purity of the sample. The results in table A.1 show that the contamination is less than $0.5 \%$. Possible hadronic interactions have cross sections small enough as to make contamination insignificant.

\footnotetext{
${ }^{c}$ One event in every 100 which enters the L3 trigger is allowed to pass without examining the physics criteria of that event. These are mainly used for diagnostic purposes in the trigger system. These events are stored in the central database in the DigiL3Prescale list.
} 


\begin{tabular}{|c|c|c|c|c|c|c|}
\hline & $\begin{array}{c}\text { MC } \\
\text { sample size }\end{array}$ & $\begin{array}{c}\text { initial } \\
\sigma(\mathrm{nb})\end{array}$ & $\begin{array}{c}\text { events } \\
\text { passing cuts }\end{array}$ & $\begin{array}{c}\text { passing } \\
\text { loose selector }\end{array}$ & $\begin{array}{c}\text { final } \\
\sigma(\mathrm{pb})\end{array}$ & contamination \\
\hline$\mu \mu \gamma$ & $903 \mathrm{~K}$ & 0.827 & 16676 & 16236 & 14.9 & \\
\hline$e^{+} e^{-} \gamma$ & $1.32 \mathrm{M}$ & 28.3 & 163 & 0 & $<0.058$ & $<0.44 \%$ \\
\hline$\tau^{+} \tau^{-}$ & $6.76 \mathrm{M}$ & 0.914 & 2 & 0 & $<3.8 \times 10^{-4}$ & $<2.7 \times 10^{-3} \%$ \\
\hline
\end{tabular}

Table A.1: Contamination in dilepton samples

While the efficiency for selecting the unbiased muons from the $\mu \mu \gamma$ Monte Carlo is only $1.6 \%$, many of the events do not produce tracks which help to generate the efficiency tables. The current efficiency tables only extend up to $4.0 \mathrm{GeV} / \mathrm{c}$, so tracks with greater momentum in the control sample are superfluous. However, they have not been cut from the sample so that the possibility of extending the tables exists. Since $97 \%$ of the events have both tracks with momentum $>4 \mathrm{GeV} / \mathrm{c}$, this low efficiency does not represent a significant loss of tracks which are of interest in generating the efficiency tables.

\section{A.4 Event Rate}

A total of 24,729 unbiased muon tracks are selected in the $\mu \mu \gamma \mathrm{MC}$ sample used. This corresponds to a rate of 11.3 tracks per $\mathrm{pb}^{-1}$. The integrated luminosity for on and off-peak data in Run 1 is $22 \mathrm{fb}^{-1}$. A total of 308,258 unbiased muon tracks are selected

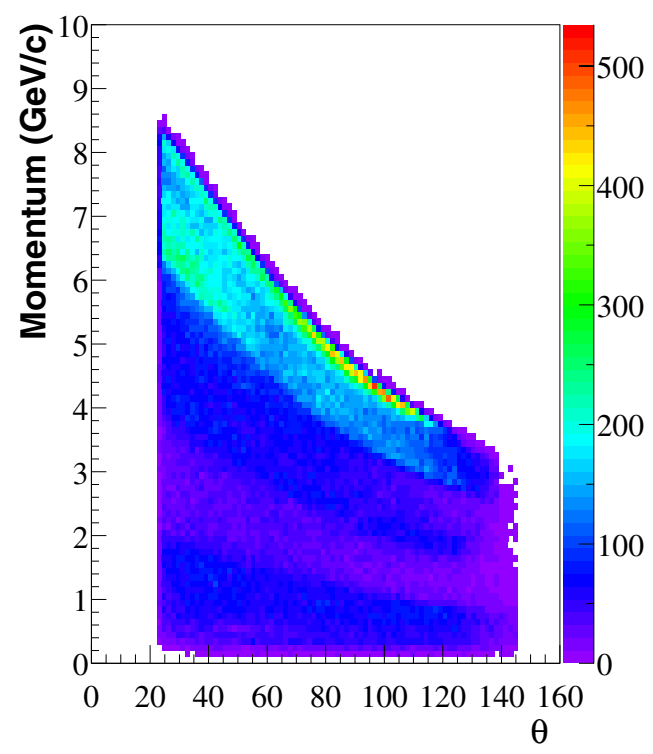

(a) full Run 1 data

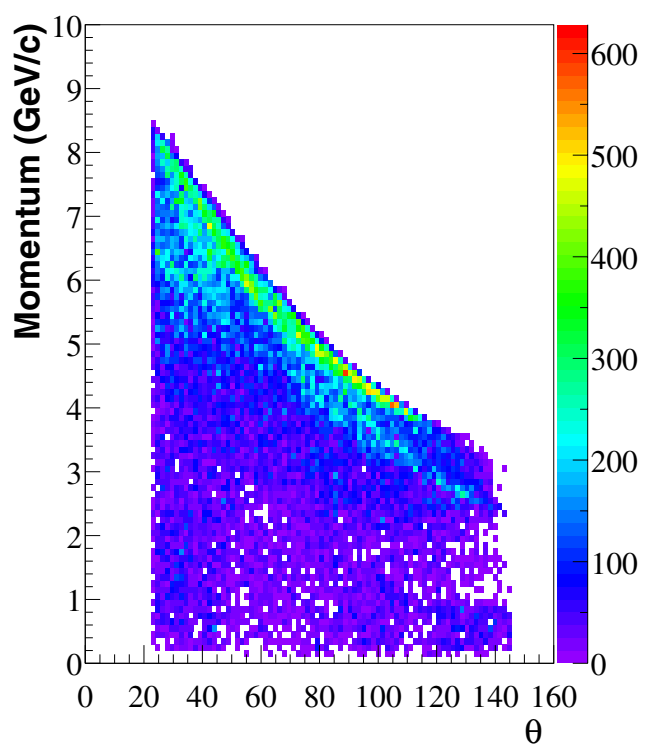

(b) SP3 MC

Figure A.1: Momentum vs $\theta$ of unbiased muon tracks from data and MC. 
from the data, corresponding to a rate of 14.0 tracks per $\mathrm{pb}^{-1}$. Plots of their distribution in $p$ and $\theta$ for both data and MC are shown in Fig. A.1. Both the data and MC show good coverage of momentum and $\theta$.

\section{A.5 Monte Carlo Comparisons}

While table A.1 shows the purity to be better than $99.5 \%$, there is noticable disagreement between the data and MC. Figure A.2 shows a significant difference in the momentum and $\theta$ spectra for unbiased muon tracks. The MC is scaled to the total entries in the data histograms. This aspect of the sample is not well understood and merits further study. It is likely that the modeling of the $\mu \mu \gamma \mathrm{MC}$ needs to be improved.

\section{A.6 $\mu$ ID Efficiencies}

The efficiencies for each of the five muon selectors were calculated using the $\mu \mu \gamma$ sample. Fig. A. 3 shows the efficiencies in momentum and $\theta$ bins for each selector. The $\phi$ bins are integrated for these plots. Of even greater interest is the comparison of efficiencies with

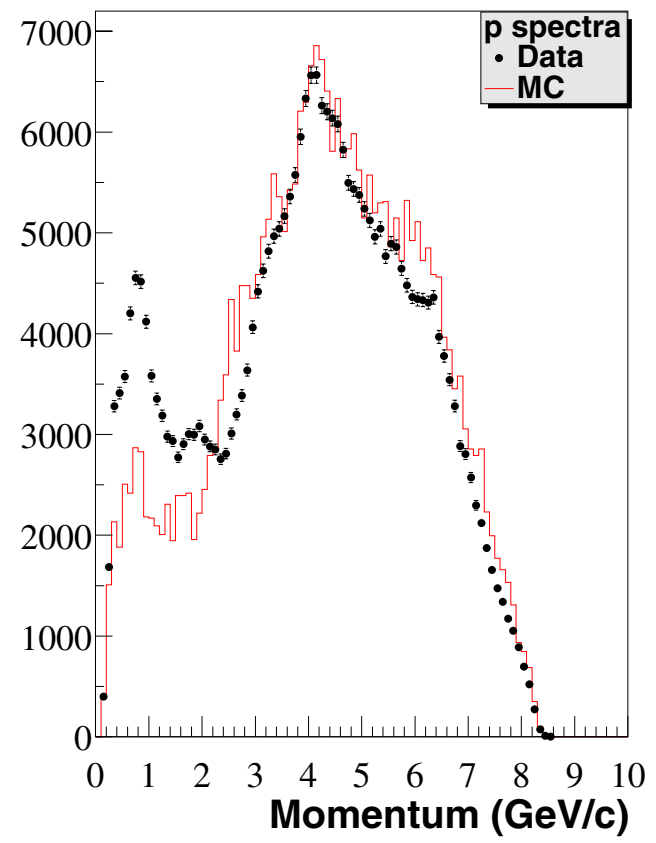

(a) Momentum spectra

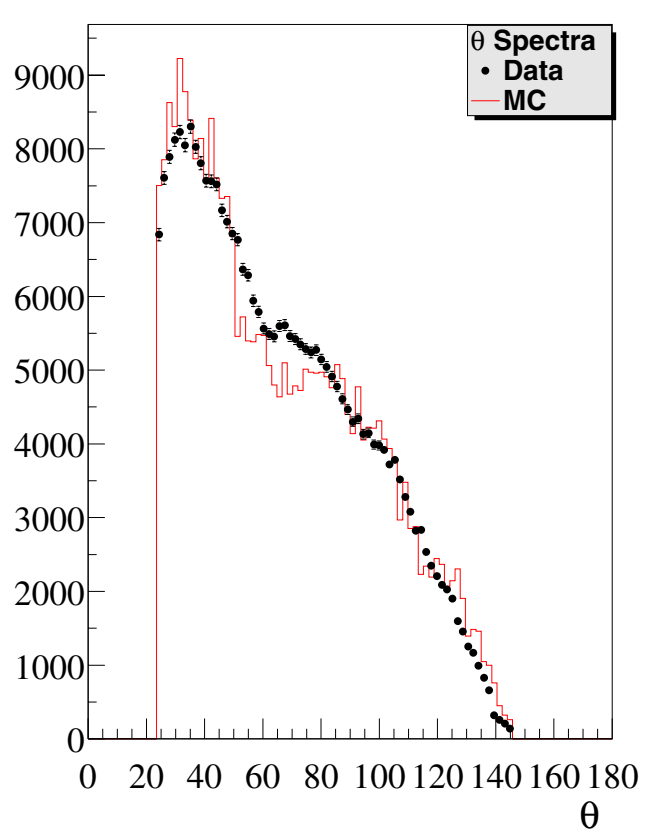

(b) $\theta$ spectra

Figure A.2: Spectra of unbiased muon tracks in data and MC. 
those from the $е e \mu \mu$ sample. Fig. A.4 shows the absolute difference between the $\mu \mu \gamma$ and $e e \mu \mu$ efficiencies for each bin. The value of each bin represents $\mu \mu \gamma-e e \mu \mu$ values, so positive values indicate that the PID table has lower efficiencies in those bins than this analysis. Fig. A.5 shows the number of entries per bin in the $\mu \mu \gamma$ sample to show the reliability of the readings. Bins with less than 30 entries are left blank in the efficiency plots since the errors are too large to provide a good comparison.

Of particular interest is the region at lower momentum in the forward endcap. The PID table efficiencies are significantly lower than those in the $\mu \mu \gamma$ sample. This can be seen in all selectors, including the minimum ionizing particle (MIP) selector. In the $\mu \mu \gamma$ sample, the efficiencies for this selector are virtually $100 \%$ for all bins, while the $e e \mu \mu$ efficiencies show a fall-off in efficiency at low $p$ and $\theta$. Since the only cut for this selector is $E_{c a l}<500 \mathrm{MeV}$, there are clearly some tracks in the ee $\mu \mu$ sample that are depositing too much energy in the EMC in this region. Since other types of particles tend to deposit more than the minimum ionizing energy, contamination would cause the efficiency to fall. Since the $\mu \mu \gamma$ sample shows excellent efficiency in all bins for the MIP selector, it indicates that there is some contamination in the ee $\mu \mu$ sample at lower momentum and forward angles which should be investigated. However, given that this region of phase space also corresponds to the most significant disagreement between data and MC, as seen in Fig. A.2, this conclusion is weakened.

A more detailed look at the efficiency comparison between the two samples is shown in figs. A.6-A.10. While the $\mu \mu \gamma$ efficiencies are not consistently below the ee $\mu \mu$ values, a significant fraction of the efficiencies are lower. This is similar to the behaviour seen in the previous $\mu \mu \gamma$ sample. Unfortunately, a direct comparison has not been made between these two $\mu \mu \gamma$ samples.

\section{A.7 Conclusion}

The new $\mu \mu \gamma$ control sample shows very good purity compared to ee $\gamma$ and $\tau \tau$ MC samples. However, the data is in potential low $p$ disagreement with the $\mu \mu \gamma \mathrm{MC}$ in both $p$ and $\theta$ spectra. In comparing the efficiencies of this control sample with the ee $\mu \mu$ efficiencies from the $\mu \mathrm{ID}$ efficiency tables, there are significant discrepancies at low momentum and forward angles for all selectors. Examination of the efficiencies for both samples indicates that the $е e \mu \mu$ sample could have some contamination in this region of phase space; however, due to the discrepancy between data and MC spectra, this is not a strong conclusion. A new module has been made in BetaPidCalib called Btamumugamma2Sample which will generate this control sample so that it can be studied further. 

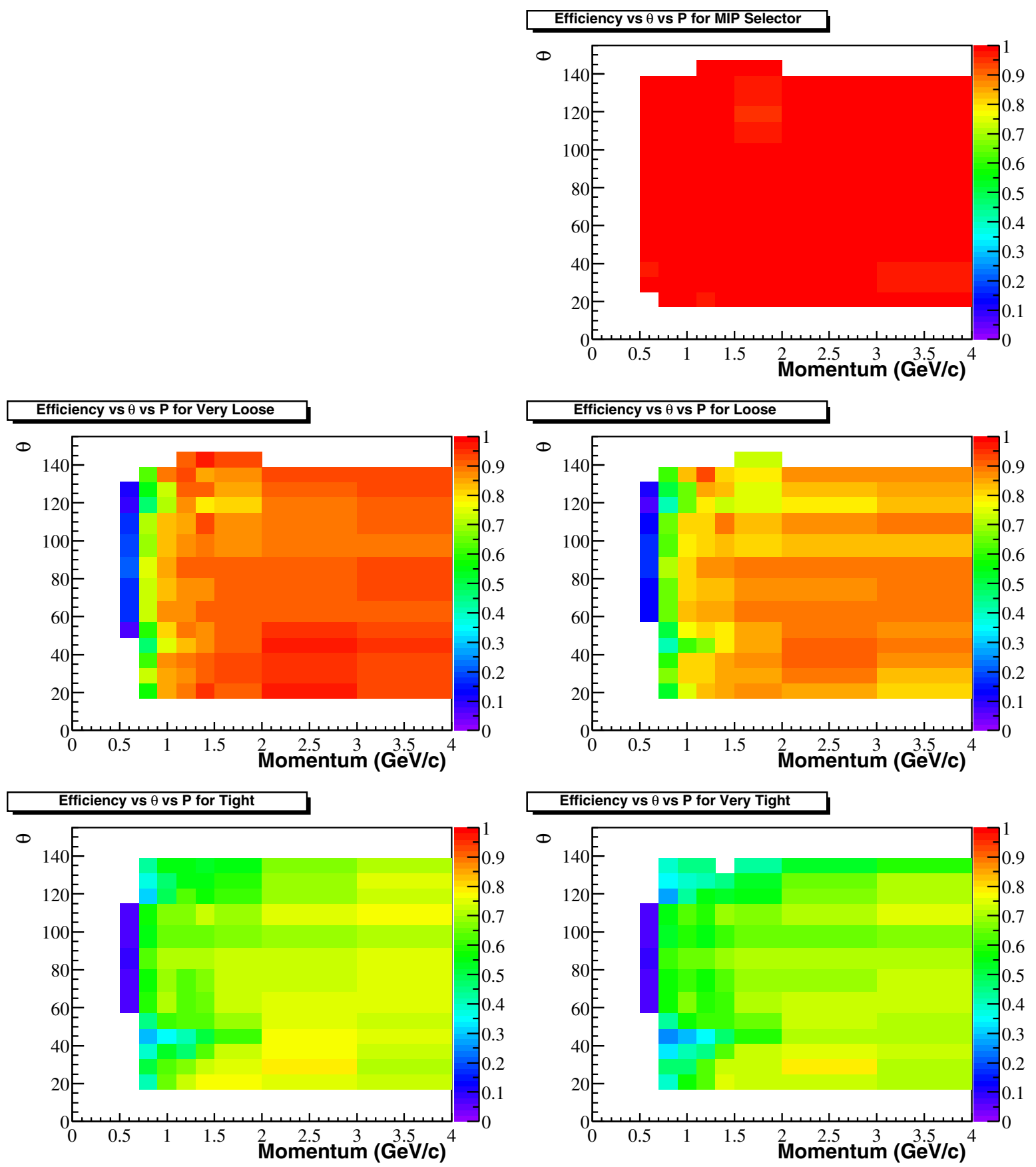

Figure A.3: $\mu \mathrm{ID}$ efficiencies for each selector calculated from the $\mu \mu \gamma$ sample. 
$\Delta$ Efficiency vs $\theta$ vs P for Very Loose

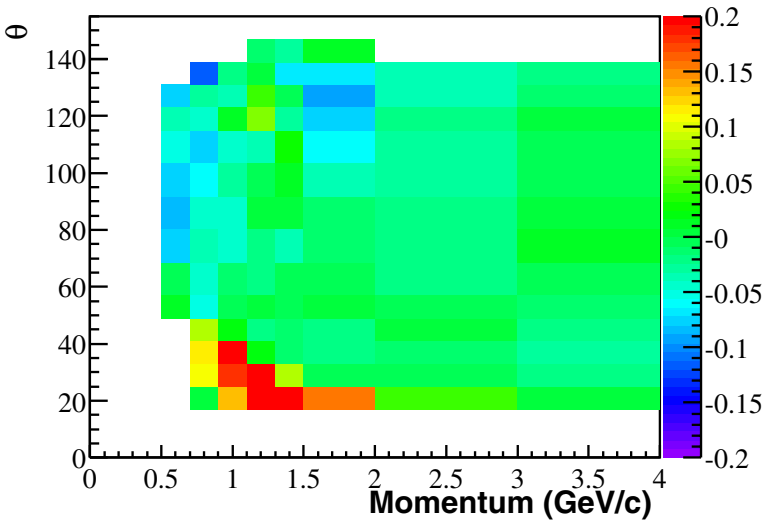

$\Delta$ Efficiency vs $\theta$ vs $\mathbf{P}$ for Tight

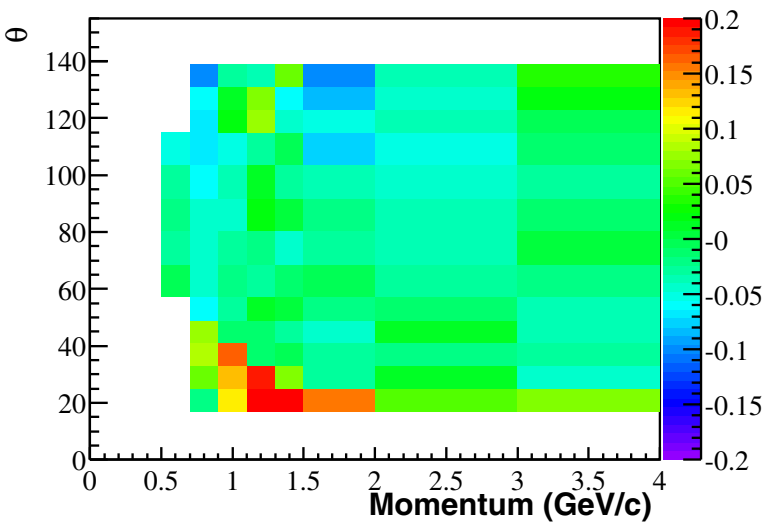

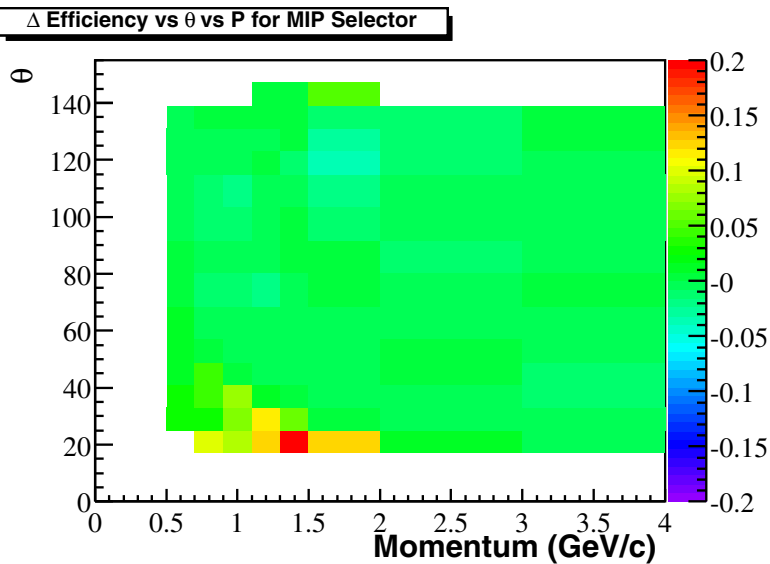

$\Delta$ Efficiency vs $\theta$ vs $\mathbf{P}$ for Loose

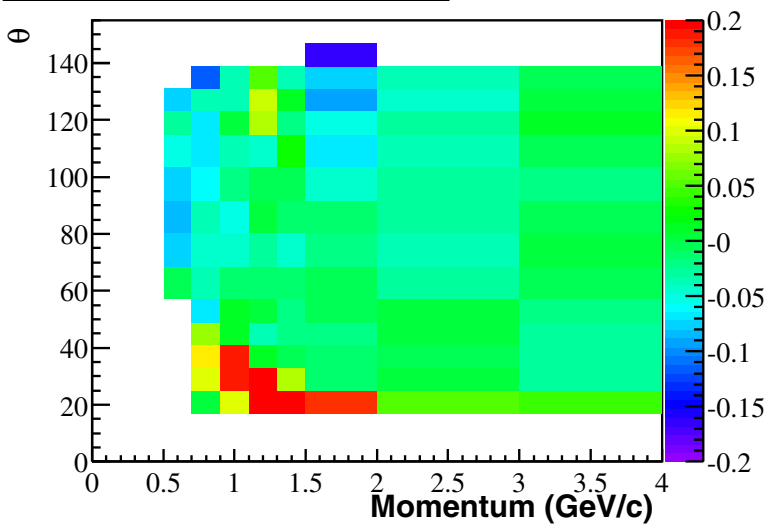

$\Delta$ Efficiency vs $\theta$ vs $\mathbf{P}$ for Very Tight

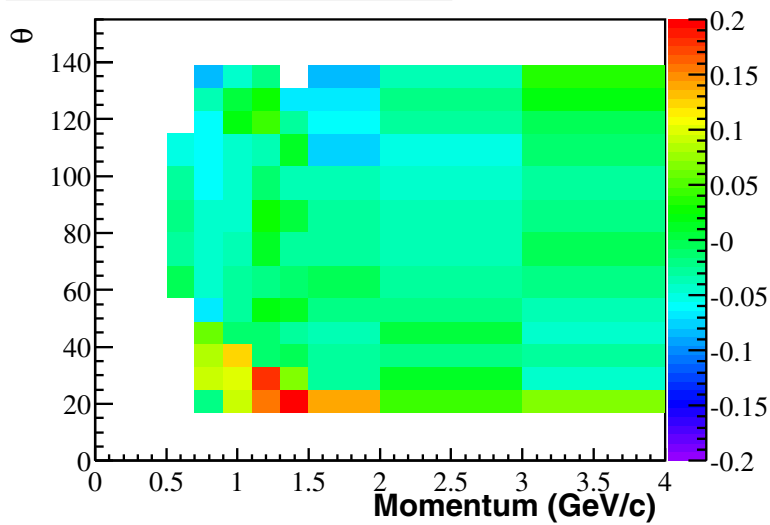

Figure A.4: Absolute efficiency differences between the $\mu \mu \gamma$ and $е e \mu \mu$ control samples. The values are calculated from $\mu \mu \gamma-e e \mu \mu$. 


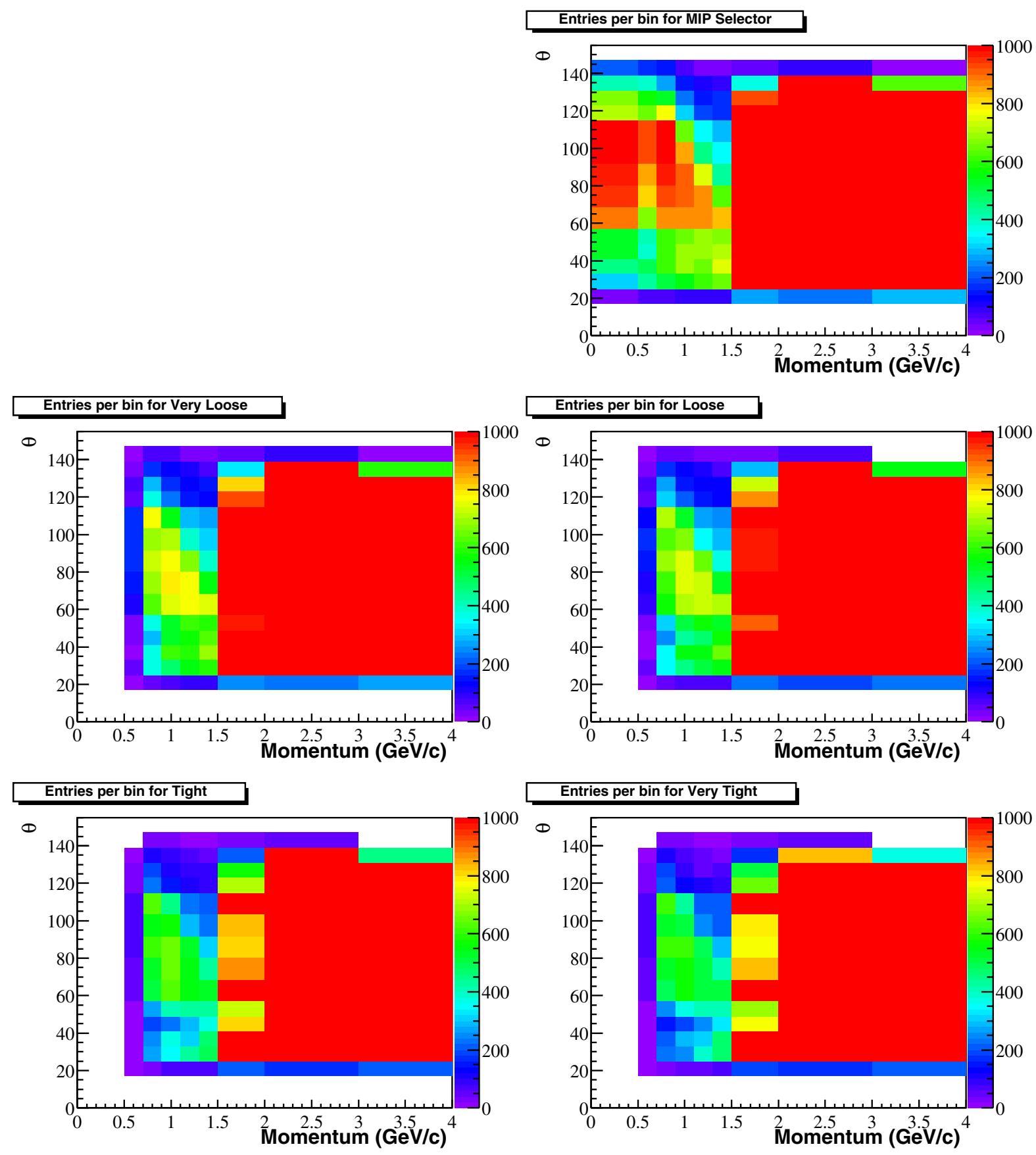

Figure A.5: Number of entries per bin in the $\mu \mu \gamma$ sample. Bins showing maximum counts could have more than 1000 entries. 

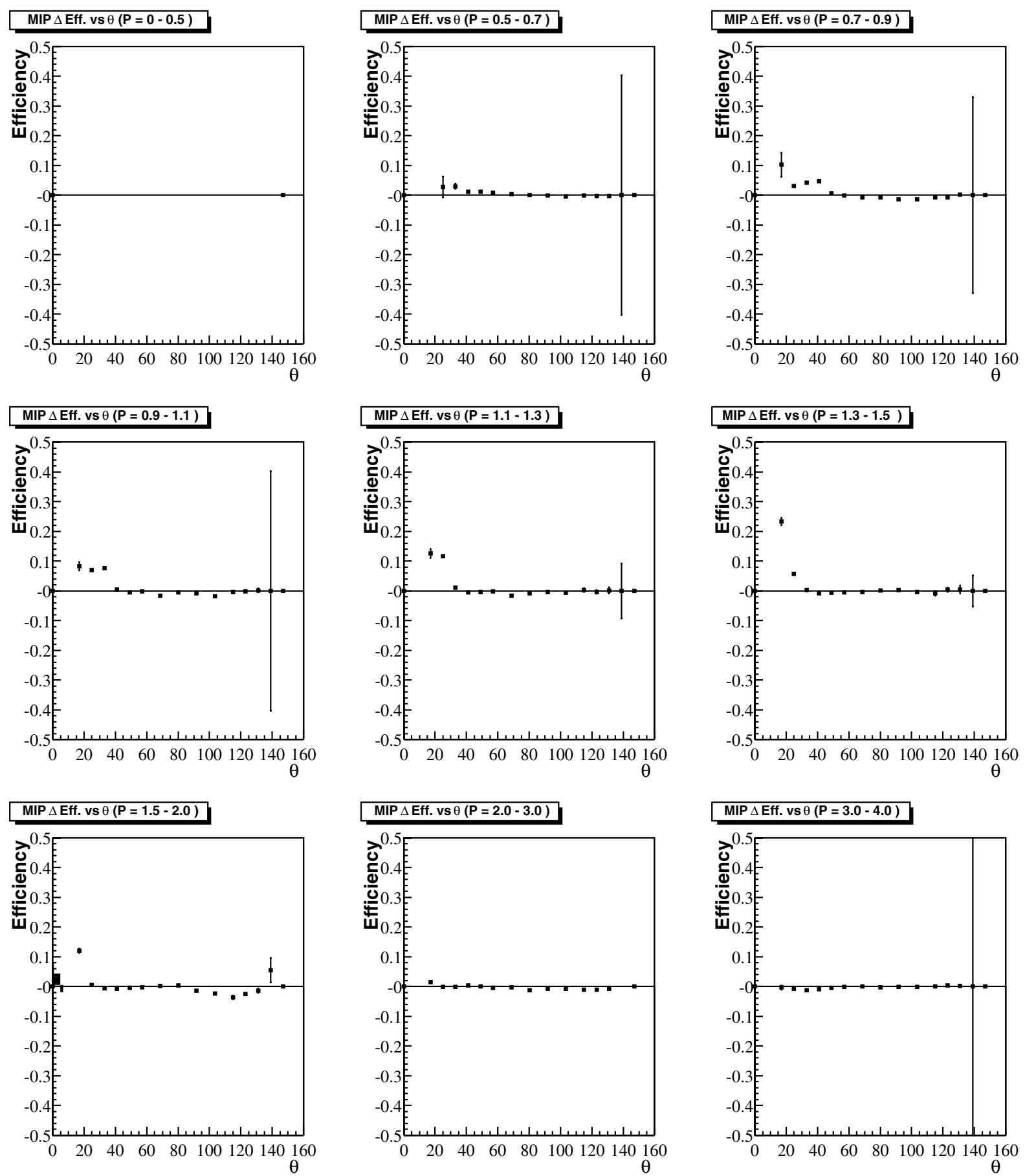

Figure A.6: Absolute efficiency differences $(\mu \mu \gamma-e e \mu \mu)$ for MIP selector. 

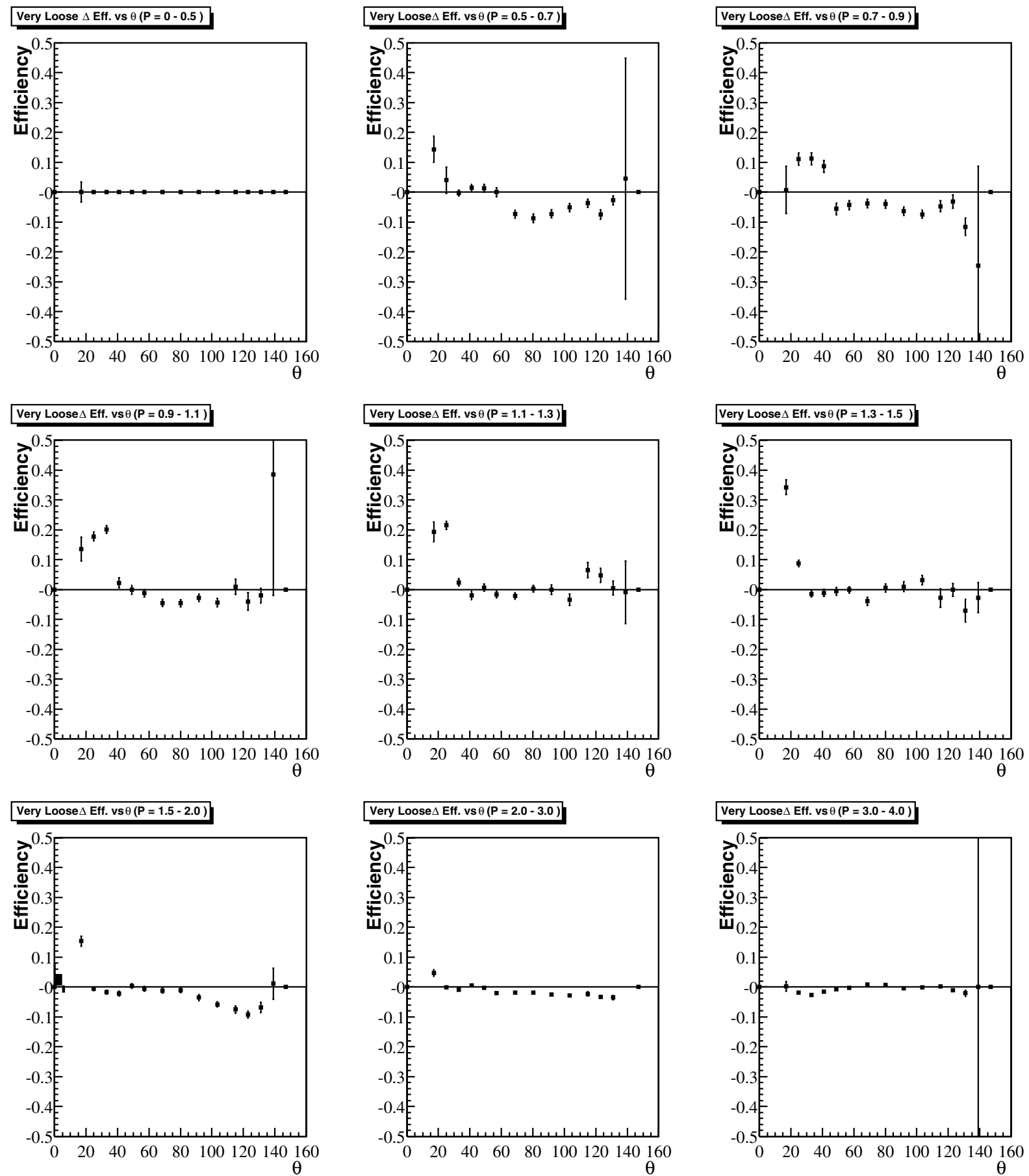

Figure A.7: Absolute efficiency differences $(\mu \mu \gamma-e e \mu \mu)$ for Very Loose selector. 

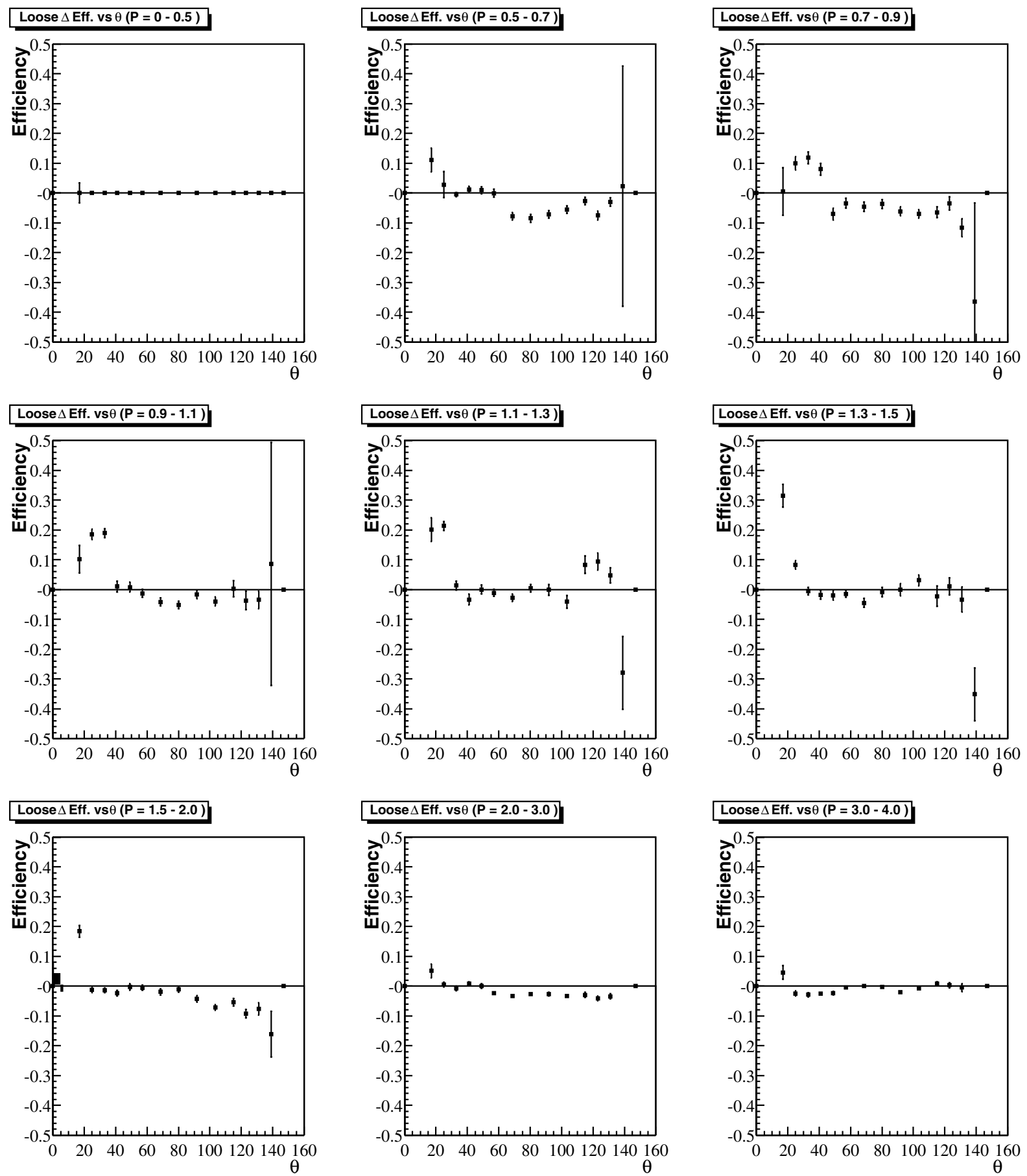

Figure A.8: Absolute efficiency differences $(\mu \mu \gamma-e e \mu \mu)$ for Loose selector. 

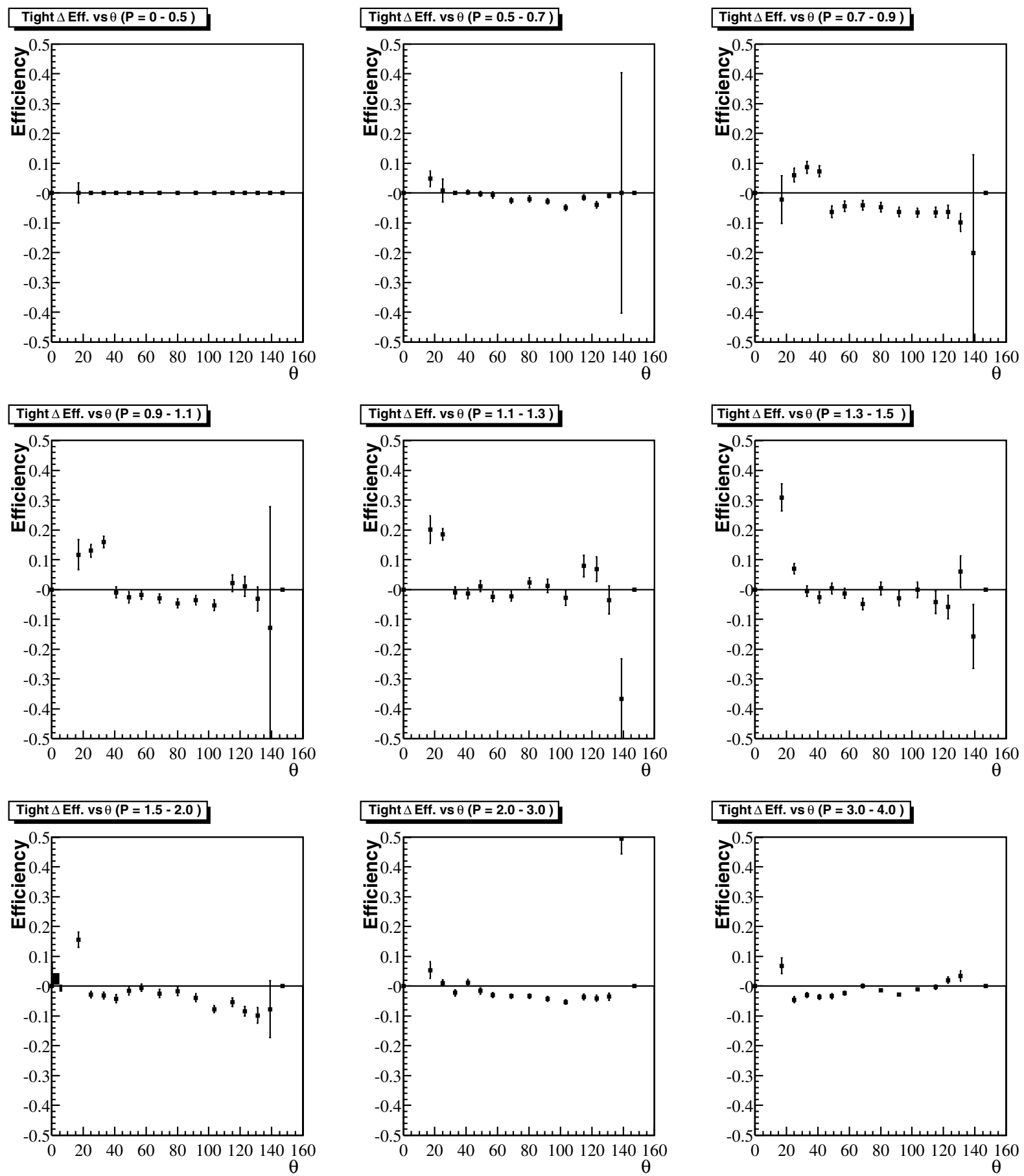

Figure A.9: Absolute efficiency differences $(\mu \mu \gamma-e e \mu \mu)$ for Tight selector. 

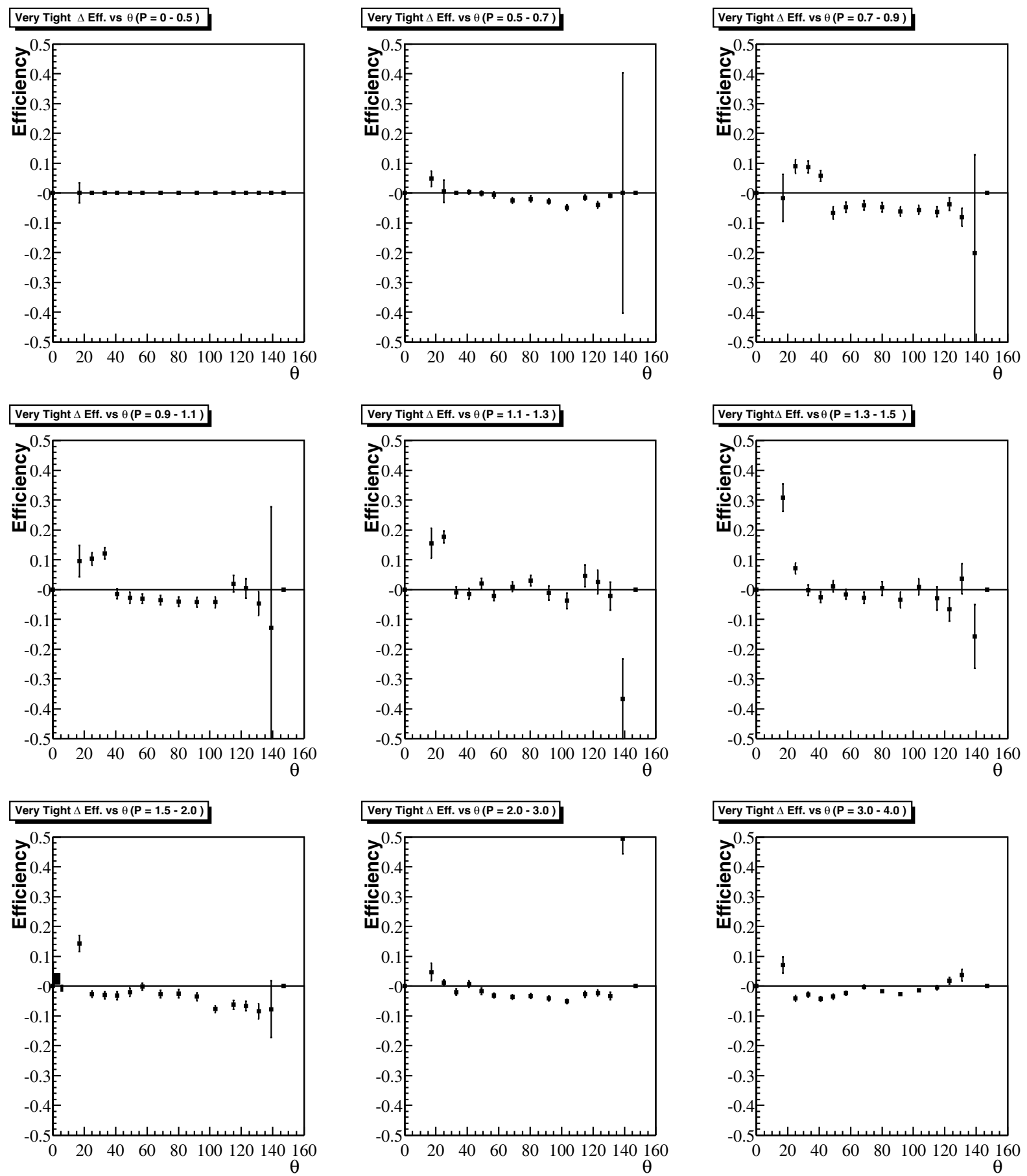

Figure A.10: Absolute efficiency differences $(\mu \mu \gamma-e e \mu \mu)$ for Very Tight selector. 


\section{Appendix B}

\section{My Contribution to the BABAR Experiment}

Because of the large size of high energy physics collaborations, it is often difficult to distinguish the contributions of an individual to the total project. For this reason, I am including a description of the work in which I personally contributed to the BABAR collaboration.

I started with BABAR in the fall of 1997. At that time, the drift chamber was under construction at TRIUMF. I assisted with the quality control for the wire stringing by helping to measure the tensions in each wire.

During the summer of 1998, I spent several months at SLAC. During this time I was helping with the BABAR database group in handling information in the offline configuration database. This database stores the configuration information about the whole detector such as calibration data, voltage settings, etc. I also performed small jobs for the DCH commissioning at SLAC. Construction of the DCH had been completed at TRIUMF, and the detector was shipped to SLAC during the spring.

Having finished my coursework in the fall of 1998, I moved to Menlo Park for the year of 1999 so that I could be more directly involved in the experiment. I joined a group working on a Java based browser for the BABAR database. This browser was designed to allow the user to search through the database for stored histograms produced during OPR. The browser would then call an external viewer to display the histograms. During this time, I also took shifts as a DCH on-call expert.

Unfortunately, the design criteria for OPR processing changed, and histograms were not stored in the main database. Therefore, the project was terminated in the summer of 2000 . However, the browser was designed so that it could be used to find other objects within the database as well. A version of this browser is in use to display information about each event, but I was not involved in this modified Java browser.

I began the $D^{*+} \mu^{-} \bar{\nu}_{\mu}$ analysis in the summer of 2000. However, my experience in data analysis of this type was still quite minimal, so I took on a smaller side project with the particle ID group to help bring me up to speed. During 2000/2001, I worked on the creation of a muon control sample based on $e^{+} e^{-} \rightarrow \mu^{+} \mu^{-} \gamma$ events, which provides a means for studying the muon ID efficiency. This sample was of sufficient quality and purity to be included in the main list of $B A B A R$ control samples. I wrote a module which 
runs during OPR processing to flag events belonging to the $\mu \mu \gamma$ control sample. Having developed the control sample and demonstrated its purity, I created muon ID efficiency tables showing the efficiency of the muon ID selectors based on the $\mu \mu \gamma$ control sample. These tables are used in muon analyses throughout the BABAR collaboration. I also wrote a BABAR Analysis Document detailing this control sample. ${ }^{[42]}$ It has been reproduced in this thesis as Appendix A.

Since completing the control sample work, I have worked exclusively on the $D^{*+} \mu^{-} \bar{\nu}_{\mu}$ analysis. I have made regular trips to SLAC to present updates of my work at BABAR collaboration meetings. I have also contributed three talks at APS and CAP conferences on the different aspects of my work for the collaboration. 


\section{Appendix C}

\section{Countries and Institutions in the BABAR collaboration}

\section{Canada}

- University of British Columbia

- McGill University

- University of Victoria

- University of Montreal

\section{China}

- Institute of High Energy Physics

\section{France}

- Ecole Polytechnique (Palaiseau)

- L.A.P.P. (Annecy)

- LPNHE Univ. Paris VI \& VII

- LAL (Orsay)

- CEA (Saclay) Particle Physics

\section{Germany}

- Ruhr University, Bochum

- Dresden Nuclear and Particle Physics Institute

- University of Heidelberg

- University of Rostock 
Italy

- INFN - Bari

- INFN - Ferrara

- INFN - Frascati

- INFN - Genova, Dipt. di Fisica

- INFN - Milan

- INFN - Naples / Universita "Federico II", Naples

- INFN - Padova

- INFN - Perugia

- INFN - Pisa

- INFN - Roma / Universita di Roma "La Sapienza"

- INFN - Torino

- INFN - Trieste

\section{Netherlands}

- NIKHEF

\section{Norway}

- University of Bergen

\section{Russia}

- Novosibirsk Institute of Nuclear Physics

\section{United Kingdom}

- University of Birmingham

- University of Bristol

- Brunel University 
- University of Edinburgh

- Imperial College (London)

- The University of Liverpool

- Manchester University

- Royal Holloway and Bedford New College, University of London

- Rutherford Appleton Laboratory

- Queen Mary and Westfield College, University of London

\section{United States}

- California Institute of Technology (Caltech)

- Colorado State University (CSU)

- Harvard University

- Lawrence Berkeley National Laboratory (LBNL)

- Lawrence Livermore National Laboratory (LLNL) HEP group

- Iowa State University (ISU)

- Ohio State University

- Prairie View A \& M University

- Massachusetts Institute of Technology Lepton-Quark Studies Group

- Rutgers University

- Princeton University

- Stanford Linear Accelerator Center (SLAC)

- Stanford University

- University of California, Irvine (UCI)

- University of California, Riverside

- University of California, Santa Barbara (UCSB)

- University of California, Santa Cruz (UCSC) 
- University of California, San Diego (UCSD)

- University of Cinncinnati

- University of Colorado, Boulder

- University of Louisville, Kentucky

- University of Maryland

- University of Massachusetts, Amherst

- University of Mississippi

- University of Notre Dame

- University of Oregon

- University of Pennsylvania

- University of Texas, Austin

- University of Texas, Dallas

- University of Wisconsin, Madison 